TECHNISCHE UNIVERSITÄT MÜNCHEN

Lehrstuhl für Analysis

\title{
Causal Localizations in Relativistic Quantum Mechanics
}

\author{
Andreas David Leiseifer
}

Vollständiger Abdruck der von der Fakultät für Mathematik der Technischen Universität München zur Erlangung des akademischen Grades eines

Doktors der Naturwissenschaften (Dr. rer. nat.)

genehmigten Dissertation.

Vorsitzender:

Univ.-Prof. Gero Friesecke, Ph. D.

Prüfer der Dissertation: 1. apl. Prof. Dr. Domenico Castrigiano

2. Univ.-Prof. Dr. Michael Marc Wolf

3. Univ.-Prof. Dr. Felix Finster

Universität Regensburg

Die Dissertation wurde am 25.02.2014 bei der Technischen Universität München eingereicht und durch die Fakultät für Mathematik am 30.06.2014 angenommen. 



\begin{abstract}
Sufficient and necessary conditions for causal localizations of massive relativistic systems are developed. It is proven that the Dirac- and the Dirac tensor-system are up to unitary equivalence the only irreducible causal localizations with finite spinor dimension which have a massive relativistic extension. A formula for this extension is given. The existence of arbitrarily good localized states of positive energy is shown. In the context of the causality condition a Paley-Wiener theorem for bounded measurable matrix-valued functions is proven.
\end{abstract}




\section{Zusammenfassung}

Hinreichende und notwendige Bedingungen für kausale Lokalisierungen massiver relativistischer Systeme werden entwickelt. Bewiesen wird, dass das Dirac- und das Dirac Tensor-System bis auf unitäre Äquivalenz die einzigen irreduziblen kausalen Lokalisierungen mit endlicher Spinor Dimension sind, die eine massive relativistische Fortsetzung besitzen. Eine Formel für diese Fortsetzung wird angegeben. Die Existenz beliebig gut lokalisierbarer Zustände positiver Energie wird gezeigt. Im Kontext der Kausalitätsbedingung wird ein Paley-Wiener Satz für beschränkte messbare matrixwertige Funktionen bewiesen. 


\section{Acknowledgements}

I would like to thank Prof. Dr. Domenico Castrigiano for entrusting me with this project. His guidance and advice in countless hours of discussions where most helpful and inspiring. Without him this thesis would not exist and I could not have hoped for a better teacher than him.

Also, I wish to thank Prof. Dr. Gero Friesecke for providing me with all necessary resources and for giving me the opportunity to teach at the university, where I found many supporting colleagues, including Yuen Au Yeung, Frauke Bäcker, Florian Drechsler, Frank Hofmaier and Moritz Simon.

Special thanks go to my family and friends for their support and trust, and to my girlfriend Andrea for her love and patience.

A. D. Leiseifer München, 2014 


\section{Notations and Conventions}

We use scalar products $\langle\cdot, \cdot\rangle$ that are linear in the second argument and anti-linear in the first argument, which is the convention used in quantum mechanics. The scalar product of $a, b \in \mathbb{R}^{n}$ is usually denoted by $a \cdot b$.

The open ball centered at $\mathbf{x} \in \mathbb{R}^{3}$ with radius $r>0$ will be written as $B_{r}(\mathbf{x})$ or simply as $B_{r}$ if $\mathbf{x}=0$.

For operators $A$ and $B$ on a Hilbert space $\mathscr{H}$ we define the commutator $[A, B]:=A B-$ $B A$ and the anti-commutator $\{A, B\}:=A B+B A$. The domain of the commutators are given by the standard rules for polynomial expressions of unbounded operators.

We primarily consider complex separable Hilbert spaces. The set of linear bounded operators acting on a Hilbert space $\mathscr{H}$ is denoted as $L(\mathscr{H})$.

Unless stated otherwise the $L^{p}$ spaces are associated with the Lebesgue measure, which is usually denoted by $\lambda$ or $d x$. Also, if no confusion is possible we will write $L^{p}$ instead of $L^{p}\left(\mathbb{R}^{d}, \mathbb{C}^{m}, \lambda\right)$, and sometimes if we define an element of $L^{p}$ by means of a function it is implicitly understood that we mean its equivalence class.

We are working in units where $c$, the velocity of light, and $\hbar$, the reduced Planck constant, are equal to 1 .

The spectrum of an operator $A$ will be denoted as $\sigma(A)$. The symbol should not be confused with the mapping $\sigma: \mathbb{R}^{3} \rightarrow \mathbb{C}^{2 \times 2}, \sigma(\mathbf{x})=\sum_{i=1}^{3} x_{i} \sigma_{i}$, where $\sigma_{i}$ are the sigma Pauli matrices. Also we use $\sigma: \mathbb{R}^{4} \rightarrow \mathbb{C}^{2 \times 2}, \sigma(x)=\sum_{\lambda=0}^{3} x_{\lambda} \sigma_{\lambda}$, where $\sigma_{0}$ is the identity matrix.

For a closed operator $T$ with a dense domain in a Hilbert space $\mathscr{H}$ we write $|T|:=\sqrt{T^{*} T}$. 


\section{A Brief Overview}

The position operator for a particle is discussed in almost every book or lecture on quantum mechanics. The axiomatic way to introduce such an observable has been established by Wightman. Soon it was discovered that the Wightman localization violates causality or suffers from negative energies unless one considers a trivial time evolution. The latter means that the energy operator, i.e. the self-adjoint generator for the time evolution, is not semi-bounded. The causality that is violated is that a particle localized in a bounded region at some given time is at any later moment in time no longer localized in any bounded region.

On the other hand, Dirac's theory, which is Lorentz covariant and contains states of negative energy, is considered to be causal, since the Dirac equation is a hyperbolic system of partial differential equations of first order.

To solve the negative energy problem Dirac proposed the Dirac sea in which all negative energy states are occupied. This, however, leads to particle interactions, thus leaving the theory. Instead one can restrict the theory to positive energy states by means of a projection. In that case the Wightman localization becomes unsharp and no particle is strictly localized.

So far, there has been no attempt to make a concrete connection between causal localizations and Dirac's theory, i.e. to show that Dirac's theory is causal in the sense that there exists a causal localization whose energy operator is the Dirac operator. We will see that the Dirac system is indeed causal. Moreover, we succeed in showing that there are further previously unknown causal localizations. We give a complete description of the irreducible causal localizations for massive systems. It turns out that they are closely related to the Dirac system - we will call them Dirac tensor systems. These are obtained by 'tensoring' the Dirac system $(V, U, E)$ with $\left(I, D^{(J)}, I\right)$, i.e. by means of

$$
V^{\prime}(t):=V(t) \otimes I \quad U^{\prime}(\mathbf{b}, B):=U(\mathbf{b}, B) \otimes D^{(J)}(B) \quad E^{\prime}(\Delta):=E(\Delta) \otimes I,
$$

where $V$ is the Dirac time-evolution, $U$ is the $I S U(2)$ representation induced from $D^{(1 / 2)} \oplus D^{(1 / 2)}, E$ is the canonical projection-valued measure and $D^{(J)}(J \geq 1 / 2)$ is a finite dimensional irreducible representation of $S U(2)$. One of our main results is that the Dirac system and the Dirac tensor systems are up to unitary equivalence the only irreducible finite causal localizations which have a massive relativistic extension.

This thesis is structured in two parts.

In part I we first review the postulates of the Wightman localization which is the notion of localization we are using primarily throughout this thesis (section 1).

The causality condition is studied in section 2, where we meet our first main result, Theorem 2.14, which can be understood as a continuation of Cas84. The theorem provides sufficient and necessary conditions for finite causal localizations. In Theorem 2.17 we show that the energy operator for every finite causal localization 
is a matrix multiplication operator corresponding to a linear function. However, this condition is not sufficient for a localization to be causal. We therefore bring relativistic causal localizations into focus.

In section 3 we review some basic facts about the representations of the Poincaré group, we introduce the Newton-Wigner localization and we derive a generalization of the BTF formulas, which connects the Newton-Wigner localization with the boost of the representation. This localization is never causal but very helpful in Lemma 4.7. where we state sufficient and necessary conditions for a localization and a time evolution to have a relativistic extension.

Another important result of section 4 is Theorem 4.9, which says that 'tensoring' a relativistic extendable causal localization with $\left(I, D^{(J)}, I\right)$ always gives another relativistic extendable causal localization.

Theorem 5.3 then refines Theorem 2.14 for relativistic causal localizations by including the linearity condition from Theorem 2.17. Applying this result to the Dirac system shows that this system is indeed a relativistic extendable causal localization. Using the 'tensoring scheme' we obtain additional relativistic causal localizations, the Dirac tensor systems. As already mentioned above, these are the only irreducible finite causal localizations which have a massive relativistic extension, see Theorem 5.10 .

Using the generalized BTF formula we obtain in Theorem 6.3 and Discussion 6.4 a canonical relativistic extension for every Dirac system and Dirac tensor system given by the boosts

$$
\mathbf{N}:=\frac{1}{2}\left\{H, \mathbf{X}^{c}\right\}+\frac{\operatorname{sgn}(H)}{|H|+C^{1 / 2}} \mathbf{P} \times\left(\mathbf{S}^{c}-\frac{1}{4 i} \mathbf{A} \times \mathbf{A}\right), \quad \mathbf{A}:=-i\left[\mathbf{X}^{c}, H\right] .
$$

In section 7 the most simple nonrelativistic causal localizations are studied.

Regarding the problem of negative energies, it is shown in section 8 that for the Dirac system and the Dirac tensor systems there exist arbitrarily good localized states of positive energy and there exist localized states with arbitrary small amount of negative energy. Moreover, due to causality, these properties remain invariant under the time evolution, see Theorem 8.8

Finally, in section 9 some open problems are discussed.

The results of part II, i.e. sections 10, 11 and 12, are more of a mathematical nature, so I devoted them their own place. They are, however, needed in part I, but including them in a linear way would result in a distraction from the main theme of the first part. 


\section{Contents}

Abstract iii

Zusammenfassung iv

\begin{tabular}{ll}
\hline Acknowledgements & v
\end{tabular}

Notations and Conventions vi

A Brief Overview vii

Contents ix

Part I

1 Localizations in Quantum Mechanics 1

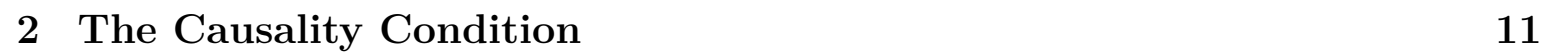

3 Finite Massive Representations of the Poincaré Group 25

$\begin{array}{lll}4 & \text { Relativistic Causal Localizations } & 36\end{array}$

5 Relativistic Extendable Causal Localizations and the Dirac System 44

$\begin{array}{|ll|}6 & \text { Relativistic Extensions for the Dirac Systems }\end{array}$

7 Non-Relativistic Causal Localizations with a Single Spin of Multi$\begin{array}{ll}\text { plicity One } & 66\end{array}$

8 Asymptotically Localized States and Hegerfeldt's Theorem 69

\begin{tabular}{lll}
\hline 9 & Open Problems & 75
\end{tabular}

Part II

\begin{tabular}{ll}
\hline 10 Paley-Wiener Theorems & 76
\end{tabular}

11 Unitary One-Parameter Groups of Matrix Multiplication Operators 89

12 Growth Conditions on $e^{i t h}$ and the Linearity of $h \quad 100$

Appendices

\begin{tabular}{|l|l|}
\hline A Projection- and Positive Operator-Valued Measures & 105
\end{tabular}

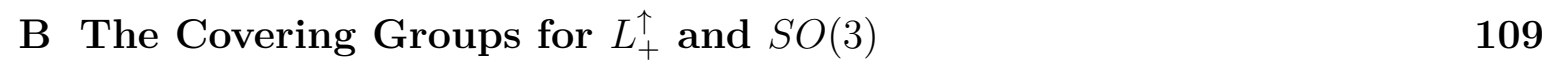


\begin{tabular}{|ll}
\hline C Finite Dimensional Representations of $S U(2)$ & 111
\end{tabular}

D Tensor Products of $S U(2)$ Representations 113

\begin{tabular}{|ll}
\hline E The Wigner $3 j$ Symbols & 116
\end{tabular}

\begin{tabular}{lll}
\hline F Causal Transformations & 119
\end{tabular}

$\begin{array}{ll}\text { G Auxiliary Lemmata } & 122\end{array}$

H Nevanlinna Theory and Related Results 128

I A Note on Schur's Lemma

\begin{tabular}{ll}
\hline References & 136
\end{tabular} 


\section{Part I}

\section{Localizations in Quantum Mechanics}

In this section we recall some results from Wig62, Cas84 and quantum mechanics.

1.1. The Postulates of the Wightman Localization. The concept of localization in quantum mechanics we use is well-known and has been introduced by Wightman Wig62. It is given by a projection-valued measure $E: \mathscr{B}\left(\mathbb{R}^{3}\right) \rightarrow L(\mathscr{H})$, where $\mathscr{B}\left(\mathbb{R}^{3}\right)$ is the Borel $\sigma$-algebra of $\mathbb{R}^{3}$ and $\mathscr{H}$ is a complex separable Hilbert space, and a projective representation (or ray representation) $U$ of the Euclidean group satisfying the covariance condition

$$
U(\mathbf{a}, R) E(\Delta) U(\mathbf{a}, R)^{-1}=E(R \Delta+\mathbf{a}), \quad \forall \mathbf{a} \in \mathbb{R}^{3}, R \in S O(3), \Delta \in \mathscr{B}\left(\mathbb{R}^{3}\right) .
$$

The definition and some properties of a projection-valued measure are given in Appendix A. As Wightman explains, replacing $\mathscr{B}\left(\mathbb{R}^{3}\right)$ by the field of sets generated by the cubes in $\mathbb{R}^{3}$ and replacing the $\sigma$-additivity of $E$ by a finite additivity leads to a notion of $E$ which can be extended to the one we are using.

This definition has the following quantum mechanical interpretations: (i) For every set $\Delta \in \mathscr{B}\left(\mathbb{R}^{3}\right)$ there exists an observable $E(\Delta)$ describing the property $\left.\right|^{1}$ of a system being localized in $\Delta$. The expectation value of $E(\Delta)$ in a given state is the probability of finding the corresponding system in $\Delta$. (ii) Every system is localized in $\mathbb{R}^{3}$. (iii) The probability of finding a system in $\Delta \cup \Delta^{\prime}$, where $\Delta$ and $\Delta^{\prime}$ are disjoint, is the sum of the probability of finding the system in $\Delta$ and the probability of finding the system in $\Delta^{\prime}$. (iv) The localization is covariant with respect to translations and rotations, i.e. the probability of finding a system in $\Delta$ equals the probability of finding the rotated and translated system in the corresponding rotated and translated $\Delta$.

The occurrence of projective representations is due to the fact that the states $\psi$ and $e^{i \alpha} \psi$, where $\alpha \in \mathbb{R}$, are physical equivalent, since they yield the same expectation values for any observable. Instead of working with projective representations of the Euclidean group one usually considers the unitary representations of its universal covering group, which is the $I S U(2)$ [[Wig62, [Bar54 and [BR86] Ch. 13 §2]. Elements of $I S U(2)$ will be written as $(\mathbf{b}, B)$, where $\mathbf{b} \in \mathbb{R}^{3}$ and $B \in S U(2)$, and the group law is given by $(\mathbf{b}, B)\left(\mathbf{b}^{\prime}, B^{\prime}\right):=\left(\mathbf{b}+B \cdot \mathbf{b}^{\prime}, B B^{\prime}\right)$, where $B \cdot \mathbf{b}:=\Lambda(B) \mathbf{b}, \Lambda: S U(2) \rightarrow S O(3)$ is the universal covering homomorphism (see Appendix $B$ ). We note that the inverse of $(\mathbf{b}, B)$ is given by $(\mathbf{b}, B)^{-1}=\left(-B^{-1} \cdot \mathbf{b}, B^{-1}\right)$.

We will only consider strongly continuous representations $U$, i.e. $\lim _{s \rightarrow e} U(s) \psi=\psi$ for every $\psi \in \mathscr{H}$, where $e$ is the identity element of the group. As noted by [[BR86]

\footnotetext{
${ }^{1}$ This is to say that $E(\Delta)$ corresponds to a yes/no measurement, in this case 'yes' means the system is inside $\Delta$ and 'no' means the system is not inside $\Delta$. According to conventional quantum theory such an observable must be an orthogonal projection.
} 
Ch. 5 §7. A.] discontinuous representations (of a locally compact group on separable Hilbert spaces) must be non-measurable [SvN50], so their physical meaning is regarded as doubtful.

To be concrete we consider the following definitions.

1.2 Definitions. Let $U$ be a strongly continuous unitary representation of $I S U(2)$ on a complex separable Hilbert space $\mathscr{H}$, and let $E: \mathscr{B} \rightarrow L(\mathscr{H})$ be a projection valued measure. Then $(U, E)$ is called a localization on $\mathscr{H}$ if the covariance condition

$$
U(s) E(\Delta) U(s)^{-1}=E(s \cdot \Delta), \quad \forall s \in I S U(2), \Delta \in \mathscr{B}\left(\mathbb{R}^{3}\right),
$$

holds, where $(\mathbf{b}, B) \cdot \Delta:=\mathbf{b}+B \cdot \Delta:=\mathbf{b}+\Lambda(B) \Delta$.

If $\left(U^{\prime}, E^{\prime}\right)$ is a localization in a Hilbert space $\mathscr{H}^{\prime}$ then $(U, E)$ and $\left(U^{\prime}, E^{\prime}\right)$ are said to be unitarily equivalent if there exists a unitary mapping $T$ of $\mathscr{H}$ onto $\mathscr{H}^{\prime}$ such that

$$
T U(s)=U^{\prime}(s) T \quad \forall s \in I S U(2) \quad \text { and } \quad T E(\Delta)=E^{\prime}(\Delta) T \quad \forall \Delta \in \mathscr{B}\left(\mathbb{R}^{3}\right) .
$$

In this case we will write $(U, E) \cong\left(U^{\prime}, E^{\prime}\right)$.

If $E$ is not a projection-valued measure but a positive operator-valued measure we will call $(U, E)$ an unsharp localization.

1.3. Discussion and Definition. Let $L$ be a strongly continuous unitary representation of $S U(2)$ on a Hilbert space $H_{0}$. Then

$$
U_{L}(\mathbf{b}, B)[g]:=\left[L(B) g\left((\mathbf{b}, B)^{-1} \cdot\right)\right],
$$

where $(\mathbf{b}, B)^{-1} x:=B^{-1} \cdot(x-\mathbf{b})$, defines a strongly continuous unitary representation of $I S U(2)$ on $\mathscr{H}:=L^{2}\left(\mathbb{R}^{3}, H_{0}\right)$, cf. [[BR86] Ch. $5 \S 1$ Example 2]. Let $E_{L}: \mathscr{B}\left(\mathbb{R}^{3}\right) \rightarrow$ $L(\mathscr{H}), E_{L}(\Delta)[g]:=\left[\chi_{\Delta} g\right]$, where $\chi_{\Delta}$ is the characteristic function of $\Delta$. Then it is easy to see that $\left(U_{L}, E_{L}\right)$ is a localization, which is unitarily equivalent to the canonical system of imprimitivity associated to the induced representation of $L$ to $I S U(2)$. The projection-valued measure $E_{L}$ is called the canonical projection-valued measure.

By Mackey's Imprimitivity Theorem [[Mac49] Theorem 2, [Fol95] Theorem 6.31] every localization is up to unitary equivalence of the form $\left(U_{L}, E_{L}\right)$. Moreover, if $L^{\prime}$ is a strongly continuous unitary representation of $S U(2)$ then $\left(U_{L}, E_{L}\right)$ is unitarily equivalent to $\left(U_{L^{\prime}}, E_{L^{\prime}}\right)$ if and only if $L$ is unitarily equivalent to $L^{\prime}$.

A localization $(U, E)$ is called finite if there exists a strongly continuous unitary representation $L$ of $S U(2)$ on a finite-dimensional Hilbert space $H_{0}$ such that $(U, E)$ is unitarily equivalent to $\left(U_{L}, E_{L}\right)$.

1.4 Discussion. Let $\mathscr{H}$ be a separable complex Hilbert space and let $E: \mathscr{B}\left(\mathbb{R}^{3}\right) \rightarrow$ $L(\mathscr{H})$ be a projection-valued measure. Put

$$
E_{i}: \mathscr{B}(\mathbb{R}) \rightarrow L(\mathscr{H}), \quad E_{i}:=E \circ \pi_{i}^{-1}, \quad \pi_{i}: \mathbb{R}^{3} \rightarrow \mathbb{R}, \quad \pi_{i}(\mathbf{x}):=\left\langle e_{i}, \mathbf{x}\right\rangle .
$$


The self-adjoint position operators $X_{i}, i=1,2,3$, corresponding to $E$ are then defined by

$$
X_{i}:=\int \operatorname{id} d E_{i}
$$

cf. [[Tha92] Sec. 1.7.3 Eq. (1.174)]. It will be convenient to use the vector notation $\mathbf{X}:=\left(X_{1}, X_{2}, X_{3}\right)^{T}$.

If $\mathscr{H}=L^{2}\left(\mathbb{R}^{3}, \mathbb{C}^{m}\right)$ and $E$ is the canonical projection-valued measure then the corresponding position operators are given by $\mathscr{D}\left(X_{i}\right)=\left\{f \in \mathscr{H}:\left[\left\langle e_{i}, \cdot\right\rangle f\right] \in \mathscr{H}\right\}$ and $X_{i}[f]=\left[\left\langle e_{i}, \cdot\right\rangle f\right]$. This is the well-known "multiplication by $x$ operator" from quantum mechanics.

We note that

$$
E(\Delta)=\prod_{i=1}^{3} E_{i}\left(\pi_{i}(\Delta)\right)
$$

for all orthotopes $\Delta \subset \mathbb{R}^{3}$. This and the following Lemma show that the correspondence $E \mapsto \mathbf{X}$ is injective, i.e. if $E^{\prime}$ is a projection-valued measure and $\mathbf{X}^{\prime}$ the corresponding position operator, then $\mathbf{X}^{\prime}=\mathbf{X}$ implies $E=E^{\prime}$.

1.5 Lemma. Let $E, E^{\prime}: \mathscr{B}\left(\mathbb{R}^{3}\right) \rightarrow L(\mathscr{H})$ be projection-valued measures on a Hilbert space $\mathscr{H}$. If $E(A)=E^{\prime}(A)$ for all orthotopes $A \subset \mathbb{R}^{3}$, then $E=E^{\prime}$.

Proof. Put

$$
\mathscr{G}:=\left\{\Delta \in \mathscr{B}\left(\mathbb{R}^{3}\right): E(\Delta)=E^{\prime}(\Delta)\right\} .
$$

Let $\mathscr{O}$ denote the set of all orthotopes in $\mathbb{R}^{3}$. Clearly, $\mathscr{O} \cup\{\varnothing\}$ is stable under finitely many intersections and we have $\mathscr{B}\left(\mathbb{R}^{3}\right)=\delta(\mathscr{O})$, where $\delta(\mathscr{O})$ is the smallest Dynkin system containing $\mathscr{O}$. Obviously $\mathbb{R}^{3} \in \mathscr{G}$. If $\Delta \in \mathscr{G}$ then

$$
E\left(\Delta^{c}\right)=I-E(\Delta)=I-E^{\prime}(\Delta)=E^{\prime}\left(\Delta^{c}\right)
$$

thus $\Delta^{c} \in \mathscr{G}$. If $\Delta_{1}, \Delta_{2}, \ldots$ is a sequence of mutually disjoint sets in $\mathscr{G}$, then for every $f \in \mathscr{H}$ we have

$$
E\left(\cup_{n} \Delta_{n}\right) f=\sum_{n} E\left(\Delta_{n}\right) f=\sum_{n} E^{\prime}\left(\Delta_{n}\right) f=E^{\prime}\left(\cup_{n} \Delta_{n}\right) f
$$

hence $\cup_{n} \Delta_{n} \in \mathscr{G}$. Thus $\mathscr{G}$ is a Dynkin system. And since $\mathscr{O}$ is a subset of $\mathscr{G}$ we have $\mathscr{B}\left(\mathbb{R}^{3}\right)=\delta(\mathscr{O}) \subset \mathscr{G} \subset \mathscr{B}\left(\mathbb{R}^{3}\right)$. Therefore $E=E^{\prime}$.

1.6. The coordinate space representation. Let $\Omega$ be a finite subset of $\mathbb{N}_{0} / 2$, let $\mathscr{H}:=\bigoplus_{j \in \Omega} \nu_{j} L^{2}\left(\mathbb{R}^{3}, \mathbb{C}^{2 j+1}\right)$, where $\nu_{j} \in \mathbb{N}$ and let $U: I S U(2) \rightarrow L(\mathscr{H})$,

$$
U:=\bigoplus_{j \in \Omega} \nu_{j} U_{D^{(j)}}
$$

where $D^{(j)}: S U(2) \rightarrow L\left(\mathbb{C}^{2 j+1}\right)$ are the standard irreducible strongly continuous unitary representations of $S U(2)$, cf. [Cas84] Eq. (6)]. 
We note that

$$
\bigoplus_{j \in \Omega} \nu_{j} L^{2}\left(\mathbb{R}^{3}, \mathbb{C}^{2 j+1}\right) \cong L^{2}\left(\mathbb{R}^{3}, \underset{j \in \Omega}{\oplus} \nu_{j} \mathbb{C}^{2 j+1}\right)
$$

and we identify these spaces.

Since $S U(2)$ is a compact group, every strongly continuous representation of $S U(2)$ is unitarily equivalent to a direct sum of irreducible unitary representations. This and the Imprimitivity Theorem (see Discussion 1.3) thus implies that every finite localization is up to unitary equivalence of the form $(U, E)$, where $E$ is the canonical projection-valued measure on $\mathscr{H}$. We call this form of a finite localization its coordinate space representation.

As an orthonormal basis for the sub-blocks $\mathbb{C}^{2 j+1}$ we choose the $S U(2)$ standard basis $\{|j, s\rangle\}_{s \in[j]}$, where $[j]:=\{-j,-j+1, \ldots, j\}$, as explained in Appendix C. This basis then determines in a canonical way an orthonormal basis for $\oplus_{k \in \Omega} \nu_{k} \mathbb{C}^{2 k+1}$ which will be written as $\{|j, \iota, s\rangle\}_{j, \iota, s}$. Here the first index $j$ is called the spin, the second index $\iota \in\left\{1, \ldots, \nu_{j}\right\}$ corresponds to the multiplicity and the third index $s$ is called the helicity index. We note that

$$
\left\langle k, \kappa, r\left|\oplus_{l \in \Omega} \nu_{l} D^{(l)}(B)\right| j, \iota, s\right\rangle=B_{11}^{j+s} B_{22}^{j-s} \delta_{k j} \delta_{\kappa \iota} \delta_{s r}
$$

for $k \in \Omega, r \in[k]$, whenever $B$ is diagonal.

A function $g \in \mathscr{H}$ can be written as $g=\sum_{j, \iota, s} g_{j, \iota, s}|j, \iota, s\rangle$, where the component functions $g_{j, \iota, s} \in L^{2}\left(\mathbb{R}^{3}, \mathbb{C}\right)$ are given by $g_{j, \iota, s}(\mathbf{x}):=\langle j, \iota, s \mid g(\mathbf{x})\rangle$.

1.7. The momentum space representation. Let $\mathscr{H}:=\oplus_{j \in \Omega} \nu_{j} L^{2}\left(\mathbb{R}^{3}, \mathbb{C}^{2 j+1}\right)$ and let $\mathscr{F}: \mathscr{H} \rightarrow \mathscr{H}$ be the Fourier transform (acting on each component separately in the usual way). The Fourier transform of an operator $T$ in $\mathscr{H}$ is given by $\hat{T}:=\mathscr{F} T \mathscr{F}^{-1}$. The momentum space representation is then the Fourier transform of the coordinate space representation. We have

$$
\hat{U}_{D^{(j)}}(\mathbf{b}, B)[f]=\left[e^{-i\langle\mathbf{b}, \cdot\rangle} D^{(j)}(B) f\left(B^{-1} \cdot\right)\right] .
$$

Operators in the momentum space representation are usually denoted with a hat to distinguish them from their coordinate space representation.

1.8. The helicity representation. Given the momentum space representation consider the unitary transform

$$
X:=\bigoplus_{j \in \Omega} \nu_{j} X^{(j)},
$$

where $X^{(j)} \in L\left(L^{2}\left(\mathbb{R}^{3}, \mathbb{C}^{2 j+1}\right)\right), X^{(j)}[f]:=\left[D^{(j)}\left(B(\cdot)^{-1}\right) f\right]$ and $B: \mathbb{R}^{3} \rightarrow S U(2)$ is a measurable map satisfying

$$
B(\mathbf{p}) \cdot e_{3}=\mathbf{p} /|\mathbf{p}| \quad \forall \mathbf{p} \in \mathbb{R}^{3} \backslash\{0\} .
$$

As in [Cas84] we will use the so-called helicity section: For $\mathbf{p} \in \mathbb{R}^{3} \backslash\left\{\alpha e_{3}: \alpha \in \mathbb{R}\right\}$ put

$$
B(\mathbf{p}):=\left(\begin{array}{cc}
a_{+} & -b^{*} a_{-} \\
b a_{-} & a_{+}
\end{array}\right), \quad a_{ \pm}:=\left(\frac{|\mathbf{p}| \pm p_{3}}{2|\mathbf{p}|}\right)^{1 / 2}, \quad b:=\frac{p_{1}+i p_{2}}{\left|p_{1}+i p_{2}\right|} .
$$


For $\alpha \geq 0$ we put $B\left(\alpha e_{3}\right):=I$ and for $\alpha<0$ we define $B\left(\alpha e_{3}\right):=\left(\begin{array}{rr}0 & -1 \\ 1 & 0\end{array}\right)$. Note that $B(\cdot)$ is continuous on $\mathbb{R}^{3} \backslash\left\{\alpha e_{3}: \alpha \leq 0\right\}$ and

$$
B(\alpha \mathbf{p})=B(\operatorname{sgn}(\alpha) \mathbf{p}), \quad B(\mathbf{p})^{-1} \cdot \mathbf{p}=|\mathbf{p}| e_{3} \quad \forall \alpha \in \mathbb{R}, \mathbf{p} \in \mathbb{R}^{3} .
$$

If $\hat{T}$ is an operator in $\mathscr{H}$ the superscript $h$ will denote its helicity transform, i.e. $T^{h}:=X \hat{T} X^{-1}$. The helicity representation is then the helicity transform of the momentum space representation. It is easy to see that

$$
U^{h}(\mathbf{b}, B) f_{j, \iota, s}|j, \iota, s\rangle=\left[e^{-i\langle\mathbf{b}, \cdot\rangle} D^{(j)}(R(\cdot, B)) f_{j, \iota, s}\left(B^{-1} \cdot\right)\right]|j, \iota, s\rangle,
$$

where $R(\mathbf{p}, B):=B(\mathbf{p})^{-1} B B\left(B^{-1} \cdot \mathbf{p}\right)$ is the Wigner rotation.

Let $\mathbf{p} \neq 0$. Since $R(\mathbf{p}, B) \cdot e_{3}=e_{3}, R(\mathbf{p}, B)$ must be diagonal. Thus $D^{(j)}(R(\mathbf{p}, B))$ is diagonal. Hence

$$
U^{h}(\mathbf{b}, B) f_{j, \iota, s}|j, \iota, s\rangle=\left[e^{-i\langle\mathbf{b}, \cdot\rangle} \kappa(\cdot, B)^{2 s} f_{j, \iota, s}\left(B^{-1} \cdot\right)\right]|j, \iota, s\rangle,
$$

where $\kappa(\mathbf{p}, B):=R(\mathbf{p}, B)_{11}$, cf. [[Cas84] (7)]. We also note that $|\kappa(\mathbf{p}, B)|=1$ for all $\mathbf{p} \in \mathbb{R}^{3}, B \in S U(2)$. Thus $U^{h}$ transforms components with the same helicity in the same way, hence the name helicity representation.

1.9 Lemma. Let $\mathbf{p} \in \mathbb{R}^{3} \backslash\{0\}$ and let $B, B^{\prime} \in S U(2)$. Then

$$
\kappa(\mathbf{p}, B) \kappa\left(B^{-1} \cdot \mathbf{p}, B^{\prime}\right)=\kappa\left(\mathbf{p}, B B^{\prime}\right) .
$$

Moreover, if $B \in S U(2)$ is diagonal, then $R(\mathbf{p}, B)=B$ for all $\mathbf{p} \in \mathbb{R}^{3} \backslash\left\{\alpha e_{3}: \alpha \leq 0\right\}$.

Proof. Let $\mathbf{p} \in \mathbb{R}^{3} \backslash\{0\}$. Since $R(\mathbf{p}, B)$ is diagonal for all $B \in S U(2)$, we have

$$
\begin{aligned}
\kappa(\mathbf{p}, B) \kappa\left(B^{-1} \cdot \mathbf{p}, B^{\prime}\right) & =R(\mathbf{p}, B)_{11} R\left(B^{-1} \cdot \mathbf{p}, B^{\prime}\right)_{11}=\left(R(\mathbf{p}, B) R\left(B^{-1} \cdot \mathbf{p}, B^{\prime}\right)\right)_{11} \\
& =R\left(\mathbf{p}, B B^{\prime}\right)_{11}=\kappa\left(\mathbf{p}, B B^{\prime}\right) .
\end{aligned}
$$

We can write, cf. [Wig62 (4.36) fol.],

$$
B(\mathbf{p})=2^{-1 / 2}\left(1+\frac{\mathbf{p} \cdot e_{3}}{|\mathbf{p}|}\right)^{-1 / 2}\left(1+\frac{\mathbf{p} \cdot e_{3}}{|\mathbf{p}|}-i \frac{\sigma\left(e_{3} \times \mathbf{p}\right)}{|\mathbf{p}|}\right),
$$

for all $\mathbf{p} \in \mathbb{R}^{3} \backslash\left\{\alpha e_{3}: \alpha \leq 0\right\}$. Then, using

$$
B \sigma\left(e_{3} \times B^{-1} \mathbf{p}\right)=\sigma\left(\left(B e_{3}\right) \times \mathbf{p}\right) B \quad \forall \mathbf{p} \in \mathbb{R}^{3}
$$

and the fact that a diagonal $B$ leaves $e_{3}$ invariant, it is easy to see that $R(\mathbf{p}, B)=B$ for all diagonal $B \in S U(2)$ and all $\mathbf{p} \neq-|\mathbf{p}| e_{3}$.

1.10 Definition. An Operator $T$ in $L^{2}\left(\mathbb{R}^{d}, \mathbb{C}^{m}\right)$ of the form $T[f]=[A f]$, where $A: \mathbb{R}^{d} \rightarrow \mathbb{C}^{m \times m}$ is a measurable matrix-valued function, will be called a matrix multiplication operator. If $T$ is a bounded matrix multiplication operator then the next Lemma shows that $A$ can be assumed to be bounded. 
1.11 Lemma. Let $T$ be a matrix multiplication operator in $L^{2}\left(\mathbb{R}^{d}, \mathbb{C}^{m}\right)$ and let $A$ : $\mathbb{R}^{d} \rightarrow \mathbb{C}^{m \times m}$ be its corresponding measurable matrix-valued function.

(a) If $T$ is bounded then $\|A(\cdot)\| \leq\|T\|$ a.e.

(b) If $A$ is essentially bounded, i.e. $\|A(\cdot)\| \leq C$ a.e. for some constant $C>0$, then $T$ is bounded.

(c) If $T$ is unitary then $A$ is unitary a.e.

Proof. (a) Suppose $T$ is bounded. Let $\left\{u_{1}, u_{2}, \ldots\right\}$ be a dense subset of the unit sphere in $\mathbb{C}^{m}$. For each $n, k \in \mathbb{N}$ put

$$
S_{n}^{(k)}:=\left\{x \in \mathbb{R}^{d}:\left\|A(x) u_{k}\right\|^{2}>\|T\|^{2}+1 / n\right\} .
$$

Suppose $0<\lambda\left(S_{n}^{(k)}\right)$. Then there exists a measurable set $T_{n}^{(k)} \subset S_{n}^{(k)}$ such that $0<$ $\lambda\left(T_{n}^{(k)}\right)<\infty$. Put $f:=\chi_{T_{n}^{(k)}} u_{k}$. But then we have

$$
\|T\|^{2} \lambda\left(T_{n}^{(k)}\right)=\|T\|^{2}\|f\|^{2} \geq\|T f\|^{2}=\int_{T_{n}^{(k)}}\left\|A(\cdot) u_{k}\right\|^{2} d \lambda \geq\left(\|T\|^{2}+1 / n\right) \lambda\left(T_{n}^{(k)}\right),
$$

which is impossible. Hence $\lambda\left(S_{n}^{(k)}\right)=0$. Since

$$
S^{(k)}:=\left\{x \in \mathbb{R}^{d}:\left\|A(x) u_{k}\right\|^{2}>\|T\|^{2}\right\}=\bigcup_{n=1}^{\infty} S_{n}^{(k)},
$$

it is a countable union of null sets, so it must be a null set, i.e. $\lambda\left(S^{(k)}\right)=0$ for all $k \in \mathbb{N}$. By the same argument $\lambda(S)=0$, where

$$
S:=\bigcup_{k=1}^{\infty} S^{(k)}
$$

If $x \in \mathbb{R}^{d}$ such that $\|A(x)\|>\|T\|$, then there exists a normalized $u \in \mathbb{C}^{m}$ such that $\|A(x) u\|>\|T\|$. By the continuity of $u \mapsto\|A(x) u\|$ there exists a $k \in \mathbb{N}$ such that

$$
\left\|A(x) u_{k}\right\|>\|T\| .
$$

Hence $x \in S$. So we must have $\|A(x)\| \leq\|T\|$ for all $x \in S^{c}$.

(b) If $A$ is essentially bounded then it is easily checked that $T$ is bounded.

(c) Let $T$ be unitary. It is $T^{*}[f]=\left[A^{*} f\right]$ and since

$$
0=\left(T^{*} T-I\right)[f]=\left[\left(A^{*} A-I_{m}\right) f\right] \quad \forall[f] \in L^{2},
$$

where $I_{m}$ is the identity operator on $\mathbb{C}^{m}$, (a) implies $\left\|A^{*}(\cdot) A(\cdot)-I\right\| \leq 0$ a.e. Hence $A^{*} A=I_{m}$ a.e. Similarly, $A A^{*}=I_{m}$ a.e. Since the union of two null sets is a null set, $A$ is unitary a.e. 
1.12 Remark. The proof of Lemma 1.11 shows that the Lemma also holds if $\mathbb{C}^{m}$ is replaced by a complex separable Hilbert space $H$ and if $T$ is an operator in $L^{2}\left(\mathbb{R}^{d}, H\right)$ such that $T[f]=[A f]$ for some measurable operator-valued function $A: \mathbb{R}^{d} \rightarrow L(H)$.

1.13 Lemma (cf. Cas84 (8)). Let $(U, E)$ be the coordinate space representation of a finite localization. Then a bounded operator $T$ commutes with $U$, i.e. $T U(s)=U(s) T$ for all $s \in I S U(2)$, if and only if there exists a bounded matrix-valued function $M$ : $\mathbb{R}_{\geq 0} \rightarrow L\left(\oplus_{j \in \Omega} \nu_{j} \mathbb{C}^{2 j+1}\right)$ such that in the helicity representation $T^{h}[f]=[M(|\cdot|) f]$ and

$$
\langle k, \kappa, r|M(\cdot)| j, \iota, s\rangle=0 \text { a.e. } \quad \forall r \neq s .
$$

Proof. The "only if" part of the Lemma: Since $\left[U^{h}(\mathbf{b}, I), T^{h}\right]=0$ for all $\mathbf{b} \in \mathbb{R}^{3}$, Lemma G.3 implies that there exists a bounded measurable matrix-valued function $A: \mathbb{R}^{3} \rightarrow L\left(\oplus_{j \in \Omega} \nu_{j} \mathbb{C}^{2 j+1}\right)$ such that $T^{h}[f]=[A f]$. Then from $\left[U^{h}(0, B), T^{h}\right]=0$ it follows that for each $B \in S U(2)$ there exists a null set $N_{B}$ such that

$$
\kappa(\mathbf{p}, B)^{2 s} A_{r s}(\mathbf{p})=\kappa(\mathbf{p}, B)^{2 r} A_{r s}\left(B^{-1} \cdot \mathbf{p}\right) \quad \forall \mathbf{p} \in N_{B}^{c},
$$

where $A_{r s}(\cdot):=\langle k, \kappa, r|A(\cdot)| j, \iota, s\rangle$ (the indices $k, j, \kappa$ and $\iota$ are fixed and will be omitted). For $r=s$ Lemma G.6 implies that

$$
A_{r r}(\cdot)=M_{r r}(|\cdot|) \quad \text { a.e. }
$$

for some measurable bounded function $M_{r r}$ on $\mathbb{R}_{\geq 0}$.

Let $l:=r-s \neq 0$ and $A:=A_{r s}$. It remains to show that $A=0$ a.e. For each $\mathbf{p} \in N_{B}^{c}$ we have

$$
A(\mathbf{p})=\kappa(\mathbf{p}, B)^{2 l} A\left(B^{-1} \cdot \mathbf{p}\right) .
$$

Put $g: \mathbb{R}^{3} \rightarrow \mathbb{C}$

$$
g(\mathbf{p}):=\int_{S U(2)} \kappa(\mathbf{p}, B)^{2 l} A\left(B^{-1} \cdot \mathbf{p}\right) d \mu(B),
$$

where $\mu$ is the Haar measure on $S U(2)$. A standard application of Fubini-Tonelli's Theorem shows that

$$
\begin{aligned}
\int_{\mathbb{R}^{3}}|g(\mathbf{p})-A(\mathbf{p})| d \mathbf{p} & \leq \int_{\mathbb{R}^{3}} \int_{S U(2)}\left|\kappa(\mathbf{p}, B)^{2 l} A\left(B^{-1} \cdot \mathbf{p}\right)-A(\mathbf{p})\right| d \mu(B) d \mathbf{p} \\
& =\int_{S U(2)} \int_{\mathbb{R}^{3}}\left|\kappa(\mathbf{p}, B)^{2 l} A\left(B^{-1} \cdot \mathbf{p}\right)-A(\mathbf{p})\right| d \mathbf{p} d \mu(B)=0 .
\end{aligned}
$$

Hence $g=A$ a.e. Let $\mathbf{p} \neq 0$. Using $\kappa\left(\mathbf{p}, B^{\prime} B\right)=\kappa\left(\mathbf{p}, B^{\prime}\right) \kappa\left(B^{\prime-1} \cdot \mathbf{p}, B\right)$, which holds for all $B, B^{\prime} \in S U(2)$, and the invariance of the Haar measure, gives

$$
\begin{aligned}
g(\mathbf{p}) & =\int_{S U(2)} \kappa\left(\mathbf{p}, B^{\prime} B\right)^{2 l} A\left(\left(B^{\prime} B\right)^{-1} \cdot \mathbf{p}\right) d \mu(B) \\
& =\kappa\left(\mathbf{p}, B^{\prime}\right)^{2 l} \int_{S U(2)} \kappa\left(B^{\prime-1} \cdot \mathbf{p}, B\right)^{2 l} A\left(B^{-1} B^{\prime-1} \cdot \mathbf{p}\right) d \mu(B)=\kappa\left(\mathbf{p}, B^{\prime}\right)^{2 l} g\left(B^{\prime-1} \cdot \mathbf{p}\right)
\end{aligned}
$$


for all $B^{\prime} \in S U(2)$. Thus

$$
g(\mathbf{p})=\kappa(\mathbf{p}, B(\mathbf{p}))^{2 l} g\left(B(\mathbf{p})^{-1} \cdot \mathbf{p}\right)=g\left(|\mathbf{p}| e_{3}\right) .
$$

Hence $g(\mathbf{p})=g\left(B^{-1} \cdot \mathbf{p}\right)$ for all $\mathbf{p} \in \mathbb{R}^{3}, B \in S U(2)$. Then Eq. (1.4) implies $g=0$ a.e., e.g., choose $B^{\prime}:=\left(\begin{array}{cc}e^{i \alpha} & 0 \\ 0 & e^{-i \alpha}\end{array}\right)$, where $\alpha \in \mathbb{R}$ such that $\kappa\left(\mathbf{p}, B^{\prime}\right)^{2 l}=e^{i 2 l \alpha} \neq 1$ for $\mathbf{p} \in \mathbb{R}^{3} \backslash\left\{\alpha e_{3}: \alpha \leq 0\right\}$. Hence $A=0$ a.e.

The "if" part of the Lemma is trivial.

1.14 Lemma (cf. Cas84 (8) fol.). Let $(U, E)$ be the coordinate space representation of a finite localization. Then $T \in L(\mathscr{H})$ commutes with $(U, E)$ if and only if there exists a matrix $M \in L\left(\oplus_{j \in \Omega} \nu_{j} \mathbb{C}^{2 j+1}\right)$ such that $T[f]=[M f]$ and

$$
\langle k, \kappa, r|M| j, \iota, s\rangle=\delta_{r s} \delta_{k j} c_{\kappa \iota}^{(k)}
$$

for some constants $c_{\kappa \iota}^{(k)} \in \mathbb{C}$. Moreover, $T^{h}=\hat{T}=T$.

Proof. Although this is an almost direct consequence of [[BR86] Ch. $16 \S 3$ Theorem 4] we will give a different proof.

Let $T$ commute with $(U, E)$. By Theorem G.1 and Lemma G.4 there exists a matrix $M \in L\left(\oplus_{j \in \Omega} \nu_{j} \mathbb{C}^{2 j+1}\right)$ such that $T[f]=[M f]$. We have

$$
D(B) M D\left(B^{-1}\right)=M \quad \forall B \in S U(2),
$$

where $D(B):=\oplus_{j \in \Omega} \nu_{j} D^{(j)}(B)$. In other words

$$
(D(B) M)_{\kappa \iota}^{(k, j)}=D(B)_{\kappa \kappa}^{(k, k)} M_{\kappa \iota}^{(k, j)}=(M D(B))_{\kappa \iota}^{(k, j)}=M_{\kappa \iota}^{(k, j)} D(B)_{\iota \iota}^{(j, j)},
$$

where $\left(M_{\kappa \iota}^{(k, j)}\right)_{r s}:=\langle k, \kappa, r|M| j, \iota, s\rangle$. Since the representations $D^{(j)}$ are irreducible and inequivalent, Schur's Lemma [[Fol95] 3.5 p. 71] implies that

$$
\langle k, \kappa, r|M| j, \iota, s\rangle=c_{\kappa \iota}^{(k)} \delta_{r s} \delta_{k j}
$$

for some constants $c_{\kappa \iota}^{(k)} \in \mathbb{C}$. Since $M$ is constant we have $\hat{T}=T$, and since $D$ commutes with $M$, it is $T^{h}=\hat{T}$.

The "if" part of the Lemma is trivial.

1.15 Definition. Let $(U, E)$ be a finite localization on a complex separable Hilbert space $\mathscr{H}$. The self-adjoint operators $P_{a}$ and $J_{a},(a=1,2,3)$, called the momentum and angular momentum operators of $U$, are defined via Stone's Theorem as

$$
U\left(s e_{a}, I\right)=\exp \left(-i s P_{a}\right), \quad U\left(0, \exp \left(i s \sigma_{a} / 2\right)\right)=\exp \left(i s J_{a}\right),
$$

where $s \in \mathbb{R}$. We will write $\mathbf{P}:=\left(P_{1}, P_{2}, P_{3}\right)^{T}$ and $\mathbf{J}:=\left(J_{1}, J_{2}, J_{3}\right)^{T}$.

1.16 Lemma. Let $(U, E)$ be a finite localization on a complex separable Hilbert space $\mathscr{H}$ and let $\mathbf{X}$ be the position operator corresponding to $E$. 
(a) There exists a dense subspace $\mathscr{D}$ of $\mathscr{H}$ such that $U \mathscr{D} \subset \mathscr{D}, \mathscr{D} \subset \mathscr{D}(A), A \mathscr{D} \subset \mathscr{D}$ and $\overline{\left.A\right|_{\mathscr{D}}}=A$ for every $A \in\{\mathbf{X}, \mathbf{P}, \mathbf{J}\}$.

(b) There is a unique bounded self-adjoint operator $\mathbf{S}$, called the spin vector for $(U, E)$, satisfying

$$
\mathbf{S}[f]=(\mathbf{J}-\mathbf{X} \times \mathbf{P})[f] \quad \forall[f] \in \mathscr{D},
$$

where $(\mathbf{A} \times \mathbf{B})_{k}:=\varepsilon_{k a b} A_{a} B_{b}$.

(c) $\mathbf{S}^{2}$ commutes with $(U, E)$.

(d) The representation $U_{D^{(j)}}$ occurs exactly $\nu$ times in the decomposition of $U$ if and only if $j(j+1)$ is an eigenvalue of $\mathbf{S}^{2}$ with multiplicity $(2 j+1) \nu$.

Proof. For (a) - (c) it suffices to consider $\left(U_{D^{(j)}}, E\right)$, where $E$ is the canonical projection valued measure.

We will show that in the momentum space representation $\mathscr{D}:=C_{c}^{\infty}\left(\mathbb{R}^{3}, \mathbb{C}^{2 j+1}\right)$ (cf. [[BR86] Ch.11 §1 (6) p. 319]) satisfies (a). In this particular representation it is clear that $\hat{U}_{D^{(j)}} \mathscr{D} \subset \mathscr{D}$ and $\mathscr{D} \subset \mathscr{D}(\hat{A})$ for $\hat{A} \in\{\hat{P}, \hat{J}\}$. Then [[RS80] Theorem VIII.11 p. 269] implies that $\mathscr{D}$ is a common core for $\hat{\mathbf{P}}$ and $\hat{\mathbf{J}}$.

To see that $\mathscr{D}$ is a core for $\hat{\mathbf{X}}$ we show that $\mathscr{D} \subset \mathscr{D}\left(\hat{X}_{a}\right)$ and $e^{i t \hat{X}_{a}} \mathscr{D} \subset \mathscr{D}$ for $a=1,2,3$. In the coordinate space representation $X_{a}$ is given by

$$
\mathscr{D}\left(X_{a}\right)=\left\{[g] \in L^{2}:\left[\left\langle\cdot, e_{a}\right\rangle g\right] \in L^{2}\right\}, \quad X_{a}[g]=\left[\left\langle\cdot, e_{a}\right\rangle g\right],
$$

(see Discussion 1.4). Clearly, the Schwartz space $\mathscr{S}\left(\mathbb{R}^{3}\right)$ is a subspace of $\mathscr{D}\left(X_{a}\right)$. Thus $\mathscr{D} \subset \mathscr{S}\left(\mathbb{R}^{3}\right)=\mathscr{F} \mathscr{S}\left(\mathbb{R}^{3}\right) \subset \mathscr{F} \mathscr{D}\left(X_{a}\right)=\mathscr{D}\left(\hat{X}_{a}\right)$. For $f \in \mathscr{D}$ we have

$$
e^{i t X_{a}} \mathscr{F}^{-1} f=\frac{1}{(2 \pi)^{3 / 2}} \int e^{i\left\langle\cdot, \mathbf{p}+t e_{a}\right\rangle} f(\mathbf{p}) d \mathbf{p}=\mathscr{F}^{-1} \tilde{f}
$$

where $\tilde{f}:=f\left(\cdot-t e_{a}\right) \in \mathscr{D}$. Hence $e^{i t \hat{X}_{a}} \mathscr{D} \subset \mathscr{D}$.

Let $f \in \mathscr{D}$. Then integration by parts gives

$$
\begin{aligned}
X_{a} \mathscr{F}^{-1} f & =\frac{1}{(2 \pi)^{3 / 2}} \int\left\langle\cdot, e_{a}\right\rangle e^{i\langle\cdot, \mathbf{p}\rangle} f(\mathbf{p}) d \mathbf{p}=\frac{1}{(2 \pi)^{3 / 2}} \int\left(-i \partial_{a} e^{i\langle\cdot, \mathbf{p}\rangle}\right) f(\mathbf{p}) d \mathbf{p} \\
& =\frac{1}{(2 \pi)^{3 / 2}} \int e^{i\langle\cdot, \mathbf{p}\rangle}\left(i \partial_{a} f\right)(\mathbf{p}) d \mathbf{p}=\mathscr{F}^{-1}\left(i \partial_{a} f\right) .
\end{aligned}
$$

Hence $\hat{X}_{a}[f]=\left[i \partial_{a} f\right]$ for all $f \in \mathscr{D}$ and $\hat{\mathbf{X}} \mathscr{D} \subset \mathscr{D}$.

The momentum operator $\hat{\mathbf{P}}$ for $\hat{U}_{D^{(j)}}$ is given by

$$
\mathscr{D}\left(\hat{P}_{a}\right)=\left\{[f] \in L^{2}:\left[\left\langle\cdot, e_{a}\right\rangle f\right] \in L^{2}\right\}, \quad \hat{P}_{a}[f]=\left[\left\langle\cdot, e_{a}\right\rangle f\right]
$$

(cf. Proposition 11.5). Clearly, $\hat{\mathbf{P}} \mathscr{D} \subset \mathscr{D}$.

We have

$$
\hat{J}_{a}[f]=\left[-\left.i \partial_{\alpha}\left(D^{(j)}\left(e^{i \alpha \sigma_{a} / 2}\right) f\left(e^{-i \alpha \sigma_{a} / 2} \cdot\right)\right)\right|_{\alpha=0}\right]
$$


for every $f \in \mathscr{D}$. Let $L_{a}$ denote the generators for $D^{(j)}$, i.e.

$$
D^{(j)}\left(e^{i \alpha \sigma_{a} / 2}\right)=e^{i \alpha L_{a}} .
$$

Then for every $f \in \mathscr{D}$ it is

$$
\hat{J}_{a}[f]=\left(\hat{S}_{a}-(\hat{\mathbf{P}} \times \hat{\mathbf{X}})_{a}\right)[f]
$$

where $\hat{S}_{a}$ is the matrix multiplication operator corresponding to $L_{a}$. This implies $\hat{J}_{a} \mathscr{D} \subset \mathscr{D}$. Clearly, $\hat{\mathbf{S}}$ is self-adjoint and bounded. Since $\left[\hat{X}_{i}, \hat{P}_{j}\right]=i \delta_{i j}$ in $\mathscr{D}$, we have $\hat{\mathbf{P}} \times \hat{\mathbf{X}}=-\hat{\mathbf{X}} \times \hat{\mathbf{P}}$. Thus $\hat{\mathbf{S}}$ satisfies (b). Because $\mathscr{D}$ is dense, $\mathbf{S}$ is the only bounded operator satisfying (b).

The well-known properties of $\mathbf{L}$ (see Lemma C.3) imply (c) and (d).

1.17 Remark. If $(U, E)$ is a finite localization then there exists a unitary $T$ commuting with $U$ such that $F:=T E T^{-1} \neq E$, e.g. for $U=U_{D^{(1 / 2)}}$ let $T^{h}[f]:=[M f]$, where

$$
\langle 1 / 2, r|M| 1 / 2, s\rangle:=\delta_{r s} e^{i s} .
$$

Plainly, $(U, F)$ is also a finite localization and in general the spin vectors for $(U, E)$ and $(U, F)$ will be different. So it is not possible to define $\mathbf{S}$ uniquely without $E$. In fact, $\mathbf{S}$ is the difference between the total angular momentum $\mathbf{J}$ and the orbital angular momentum $\mathbf{X} \times \mathbf{P}$. 


\section{The Causality Condition}

We now define and discuss causal localizations. Our first important Lemma is 2.11 which gives us a simple necessary and sufficient condition for causality. After this Lemma we need the results of Part II, i.e. sections 10, 11 and 12, to continue our discussion. We think the subjects of these sections are interesting enough to devote them their own place. Also, including them in this section would result in a distraction from the objective given here.

2.1 Definition. A continuous unitary one-parameter group $V$ on a complex separable Hilbert space $\mathscr{H}$ is a map $V: \mathbb{R} \rightarrow L(\mathscr{H})$ such that $V(t)$ is unitary for all $t \in \mathbb{R}$ and

(a) $V(0)=I$.

(b) $V(s+t)=V(s) V(t)$ for all $s, t \in \mathbb{R}$.

(c) $\lim _{t \rightarrow 0} V(t) \psi=\psi$ for every $\psi \in \mathscr{H}$.

Note that the continuity at 0 and the homomorphism property imply that $V$ is strongly continuous:

$$
\lim _{t \rightarrow s} V(t) \psi=\lim _{t \rightarrow s} V(s) V(t-s) \psi=V(s) \lim _{\tau \rightarrow 0} V(\tau) \psi=V(s) \psi
$$

for every $s \in \mathbb{R}$ and $\psi \in \mathscr{H}$.

If $(U, E)$ is a localization on a complex separable Hilbert space $\mathscr{H}$, then $V$ is said to be a time evolution if $V$ is a continuous unitary one-parameter group on $\mathscr{H}$ commuting with $U$. In quantum mechanical terms this means that if $\psi$ is the state of system at time zero, then $V(t) \psi$ is the state of system at time $t$.

2.2 Definition. We use the same notation of causality as in Cas84: Let $(U, E)$ be a localization on a complex separable Hilbert space $\mathscr{H}$ and let $V$ be a continuous unitary one-parameter group on $\mathscr{H}$. Then $(V, U, E)$ is said to be a causal localization if $V$ commutes with $U$ (i.e. $V$ is a time evolution) and if $E_{c}$, the completion of $E$ (see A.8) satisfies

$$
V(t) E_{c}(\Delta) V(-t) \leq E_{c}\left(\Delta_{t}\right), \quad \forall t \in \mathbb{R}, \Delta \in \mathscr{B}\left(\mathbb{R}^{3}\right),
$$

where

$$
\Delta_{t}:=\left\{\mathbf{y} \in \mathbb{R}^{3}: \exists \mathbf{x} \in \Delta:|\mathbf{x}-\mathbf{y}| \leq|t|\right\} .
$$

Note that we use units where $c$, the velocity of light, and $\hbar$, the reduced Planck constant, are equal to 1.

In Discussion 2.5 we explain why we need to use the completion of $E$.

We say $(V, U, E)$ is a finite causal localization, if $(V, U, E)$ is a causal localization and $(U, E)$ a finite localization.

Similar definitions apply if $E$ is a positive operator-valued measure, in that case we speak of causal unsharp localizations or finite causal unsharp localization. 
2.3. Interpretations of the causality condition. The causality condition for a projection-valued measure is a mathematical description of Einstein causality [Cas84]: Let $\psi \in \mathscr{H}$ be a state of the system at time zero and let $\psi_{t}:=V(t) \psi$ be the state of the system at time $t>0$. Suppose $\psi$ is localized in $\Delta \in \mathscr{B}\left(\mathbb{R}^{3}\right)$, i.e. $E_{c}(\Delta) \psi=\psi$. Then

$$
\begin{aligned}
0 & \leq\left\|\left(I-E_{c}\left(\Delta_{t}\right)\right) \psi_{t}\right\|^{2}=\left\langle\psi_{t},\left(I-E_{c}\left(\Delta_{t}\right)\right) \psi_{t}\right\rangle \leq\left\langle\psi_{t},\left(I-V(t) E_{c}(\Delta) V(-t)\right) \psi_{t}\right\rangle \\
& =\left\|\left(I-V(t) E_{c}(\Delta) V(-t)\right) \psi_{t}\right\|^{2}=\left\|\left(I-E_{c}(\Delta)\right) \psi\right\|^{2}=0
\end{aligned}
$$

means that $\psi_{t}$ must be localized in $\Delta_{t}$. In other words, $\psi$ cannot move faster than light.

Another way to interpret the causality condition is the following: The expectation value of finding a state of a system $\psi$ at time zero in a region $\Delta$ is given by $\left\langle\psi, E_{c}(\Delta) \psi\right\rangle$. By the causality condition it is less or equal than $\left\langle V(t) \psi, E_{c}\left(\Delta_{t}\right) V(t) \psi\right\rangle$, which is the expectation value of finding the state of system at time $t$ in the region $\Delta_{t}$. Here we do not require $E_{c}(\Delta) \psi=\psi$, thus this interpretation is appropriate when $E$ is a positive operator-valued measure.

In this way we may also consider a mixture of states

$$
T \in\{T \in L(\mathscr{H}): T \geq 0, \operatorname{tr}(T)=1\} .
$$

The probability of finding the state $T$ in $\Delta$ is given by $p_{T}(\Delta):=\operatorname{tr}\left(T E_{c}(\Delta)\right.$ ) (see [[BGL95] II.1.2 (1.21)]). We show that causality implies

$$
p_{T}(\Delta) \leq p_{T_{t}}\left(\Delta_{t}\right), \quad T_{t}:=V(t) T V(-t) .
$$

Let $t \in \mathbb{R}$ and put $V:=V(t), E:=E_{c}(\Delta)$ and $F:=E_{c}\left(\Delta_{t}\right)$. Since $T \geq 0$ and $\operatorname{tr}(T)<\infty$, $T$ is compact [[RS80] Theorem VI.21], hence, by the Hilbert-Schmidt Theorem there exists a complete orthonormal basis $\left\{\phi_{n}\right\}_{n \in \mathbb{N}}$ for $\mathscr{H}$ such that

$$
T=\sum_{n=1}^{\infty} \lambda_{n}\left\langle\phi_{n}, \cdot\right\rangle \phi_{n}
$$

where $\lambda_{n} \stackrel{n \rightarrow \infty}{\longrightarrow} 0$, in fact $\lambda_{n} \in[0,1]$ and $\sum_{n} \lambda_{n}=1$. Thus

$$
\begin{aligned}
\operatorname{tr}(T E) & =\sum_{k=1}^{\infty}\left\langle\phi_{k}, T E \phi_{k}\right\rangle=\sum_{k=1}^{\infty} \lambda_{k}\left\langle\phi_{k}, E \phi_{k}\right\rangle=\sum_{k=1}^{\infty} \lambda_{k}\left\langle V \phi_{k}, V E V^{*} V \phi_{k}\right\rangle \\
& \leq \sum_{k=1}^{\infty} \lambda_{k}\left\langle V \phi_{k}, F V \phi_{k}\right\rangle=\operatorname{tr}\left(V T V^{*} F\right) .
\end{aligned}
$$

This completes the proof.

2.4. The occurrence of negative energies. Before we go any further we want to note that there is a deep physical problem with causal localizations. If one requires the energy operator, i.e. the generator $H$ of the time evolution $V$, to be semi-bounded (positivity of energy) then $V$ commutes with $E$ [Cas84]. This implies that $V$ is a 
constant matrix multiplication operator [Cas84 (8) fol.] and, since $V(0)=I$, we must have $V(t)=I$ for all $t \in \mathbb{R}$. So in other words, any nontrivial causal localization will suffer from negative energies.

A possible way out of this is to restrict the theory onto positive energy states, i.e. by considering the Hilbert space $\mathscr{H}_{+}:=P_{+}(\mathscr{H})$, where

$$
P_{+}:=\frac{1}{2}(I+\operatorname{sgn}(H))
$$

is the projection onto positive energy states (cf. Definition 3.5). Since $H$ commutes with $V$ and $U$, it is clear that $V$ and $U$ leave $\mathscr{H}_{+}$invariant. However, the projectionvalued measure $E$ does not leave $\mathscr{H}_{+}$invariant. A natural generalization of $E$ which leaves $\mathscr{H}_{+}$invariant is $\left[\left[\mathrm{BK} 03\right.\right.$ Eq. (5)] $F: \mathscr{B}\left(\mathbb{R}^{3}\right) \rightarrow L\left(\mathscr{H}_{+}\right)$,

$$
F(\Delta):=P_{+} E(\Delta) P_{+},
$$

or more precisely, $F(\Delta):=i n^{*} E(\Delta) i n$, where $i n: \mathscr{H}_{+} \hookrightarrow \mathscr{H}$ is the inclusion map. Then $F$ is not a projection-valued measure but a positive operator-valued measure and $\left(\left.V\right|_{\mathscr{H}_{+}},\left.U\right|_{\mathscr{H}_{+}}, F\right)$ is an unsharp causal localization.

For the Dirac system, defined in 5.4, it makes sense to consider $X_{i}^{+}:=P_{+} X_{i} P_{+}$, since there is a dense subspace $\mathscr{D}$ which is invariant under $X_{i}$, the generators and $P_{+}$. Here $X_{i}$ are the position operators corresponding to $E$. In the momentum space representation we may choose $\mathscr{D}=C_{c}^{\infty}\left(\mathbb{R}^{3}\right)$ (cf. Theorem 3.10 and 6.1). However, the $X_{i}^{+}$no longer commute: We have

$$
\left.\left[X_{i}^{+}, X_{j}^{+}\right]\right|_{\mathscr{D}}=-\left.i \varepsilon_{i j k} \frac{1}{H^{2}} P_{+} S_{k} P_{+}\right|_{\mathscr{D}}
$$

see [[Tha92] Eq. (1.154)], where $\mathbf{S}=\mathbf{J}-\mathbf{X} \times \mathbf{P}$.

Hegerfeldt Heg98 notes that also unsharp causal localizations have a similar problem: Normalized states $\psi \in \mathscr{H}$ such that $\langle\psi, F(\Delta) \psi\rangle=1$ for some compact ${ }^{2}$ $\Delta \subset \mathbb{R}^{3}$ do not exist unless $\langle V(t) \psi, F(\Delta) V(t) \psi\rangle=1$ for all $t \in \mathbb{R}$. Thus no state can be expected with absolute certainty to be in a compact $\Delta$ unless it stays there forever.

For the Dirac System, and, as we will see, for every massive relativistic extendable causal localizations, there exists no nonzero state of positive energy which is localized in some bounded measurable set, see $\mathrm{Heg} 74$ and Theorem 8.1. However, in section 8 we show that in the relativistic case for every $\varepsilon>0$ and every open ball $\Delta$ there exists a state $\psi \in \mathscr{H}$ such that $P_{+} \psi=\psi,\|\psi\|=1$ and $\left\|E\left(\Delta^{c}\right) \psi\right\|<\varepsilon$. The last estimate can also be written as $\|E(\Delta) \psi\|^{2}>1-\varepsilon^{2}$, and by causality we have

$$
\left\|E\left(\Delta_{t}\right) \psi_{t}\right\|^{2}>1-\varepsilon^{2} \quad \forall t \in \mathbb{R},
$$

where $\psi_{t}:=V(t) \psi$.

\footnotetext{
${ }^{2}$ Hegerfeldt does not really specify the sets for which this result holds and we will make no sophisticated attempt here to do so. However, it is save to say that the result holds at least for compact sets.
} 
So we think this is a good reason to pursuit these concepts. Moreover, the concept of a relativistic causal localization enables us to derive the Dirac equation from first principles (see Theorem 5.10 and Discussion 5.13).

2.5 Discussion. The reason for the use of the completion of $E$ in Definition 2.2 is that we cannot exclude that $\Delta_{t} \notin \mathscr{B}\left(\mathbb{R}^{3}\right)$. In Lemma 2.6 we show that $\Delta_{t}$ is always Lebesgue measurable, and by Lemma 2.7 the null sets of $E$ are precisely the null sets of the Lebesgue measure $\lambda$ in $\mathscr{B}\left(\mathbb{R}^{3}\right)$. Thus the completion of $E$, denoted by $E_{c}$, is a projection-valued measure on the Lebesgue measurable sets satisfying

$$
E_{c}(A \cup S)=E(A),
$$

for $A \in \mathscr{B}\left(\mathbb{R}^{3}\right)$ and $S \in \mathscr{N}:=\left\{S \subset \mathbb{R}^{3}: \exists N \in \mathscr{B}\left(\mathbb{R}^{3}\right): S \subset N, \lambda(N)=0\right\}$ (see A.8 for details).

By the rotational- and translational-invariance of $\lambda$ we have $E_{c}(g \cdot S)=0$ for every $g \in I S U(2)$ and $S \in \mathscr{N}$. Thus the completion of $E$ still satisfies the covariance property: For $A \in \mathscr{B}\left(\mathbb{R}^{3}\right), S \in \mathscr{N}$ and $g \in I S U(2)$ we have

$U(g) E_{c}(A \cup S) U(g)^{-1}=U(g) E(A) U(g)^{-1}=E(g \cdot A)=E_{c}(g \cdot A \cup g \cdot S)=E_{c}(g \cdot(A \cup S))$.

It has also been noted in Wig62 that every $E$ can be extended to all Lebesgue measurable sets. Moreover, we have

$$
V(t) E_{c}(A \cup S) V(-t)=V(t) E_{c}(A) V(-t) \leq E_{c}\left(A_{t}\right) \leq E_{c}\left((A \cup S)_{t}\right),
$$

since $A_{t} \subset(A \cup S)_{t}$. Thus the causality condition also holds for every Lebesgue measurable set.

The conclusion is that we can avoid the problem $\Delta_{t} \notin \mathscr{B}\left(\mathbb{R}^{3}\right)$ by considering the completion of $E$.

2.6 Lemma. Let $\lambda$ be the Lebesgue measure on $\mathbb{R}^{3}$, let $A \subset \mathbb{R}^{3}$ and let $t \neq 0$. Then $A_{t}$ is Lebesgue measurable, $U(A, t) \subset A_{t} \subset C(A, t)$ and

$$
\lambda(C(A, t) \backslash U(A, t))=0,
$$

where

$$
U(A, t):=\left\{\mathbf{x} \in \mathbb{R}^{3}: d(\mathbf{x}, A)<|t|\right\}, \quad C(A, t):=\left\{\mathbf{x} \in \mathbb{R}^{3}: d(\mathbf{x}, A) \leq|t|\right\}
$$

are Borel sets and $d(\mathbf{x}, A):=\inf \{|\mathbf{x}-\mathbf{y}|: \mathbf{y} \in A\}$.

Proof. Clearly, we may assume that $t>0$ and $A \neq \varnothing$. Since $\mathbf{x} \mapsto d(\mathbf{x}, A)$ is continuous, $U(A, t)$ is open and $C(A, t)$ is closed, in particular they are Borel sets. It is easy to see that $U(A, t) \subset A_{t} \subset C(A, t)$. Hence, it remains to show that

$$
N(A):=C(A, t) \backslash U(A, t)=\left\{\mathbf{x} \in \mathbb{R}^{3}: d(\mathbf{x}, A)=t\right\}
$$

is a null set. 
We show that $N(A) \cap B_{\varepsilon}(\mathbf{z}) \subset N\left(A \cap B_{\varepsilon+t}(\mathbf{z})\right)$ for all $\varepsilon>0$ and $\mathbf{z} \in \mathbb{R}^{3}$. Let $\mathbf{x} \in N(A) \cap B_{\varepsilon}(\mathbf{z})$. Thus $|\mathbf{x}-\mathbf{z}|<\varepsilon$ and for every $\delta>0$ there is exists a $\mathbf{y}_{\delta} \in A$ such that $\left|\mathbf{y}_{\delta}-\mathbf{x}\right| \leq t+\delta$. If we choose $\delta<\varepsilon-|\mathbf{x}-\mathbf{z}|$ we find

$$
\left|\mathbf{y}_{\delta}-\mathbf{z}\right| \leq\left|\mathbf{y}_{\delta}-\mathbf{x}\right|+|\mathbf{x}-\mathbf{z}|<t+\delta+|\mathbf{x}-\mathbf{z}|<t+\varepsilon .
$$

This implies $\mathbf{y}_{\delta} \in A \cap B_{\varepsilon+t}(\mathbf{z})$. Hence

$$
t=d(\mathbf{x}, A) \leq d\left(\mathbf{x}, A \cap B_{\varepsilon+t}(\mathbf{z})\right) \leq\left|\mathbf{x}-\mathbf{y}_{\delta}\right| \leq t+\delta,
$$

and $\delta \rightarrow 0$ shows that $\mathbf{x} \in N\left(A \cap B_{\varepsilon+t}(\mathbf{z})\right)$.

Thus it suffices to show that $N\left(A \cap B_{\varepsilon+t}(\mathbf{z})\right)$ is a null set for every $\mathbf{z} \in \mathbb{R}^{3}$ and some fixed $\varepsilon>0$, since $\mathbb{R}^{3}$ can be covered by countable many balls of radius $\varepsilon$, and since the countable union of null sets is a null set. Moreover, because $B_{\varepsilon+t}(\mathbf{z})$ can be covered by finitely many balls of radius $t / 3$ and since $N\left(A_{1} \cup \ldots \cup A_{n}\right) \subset N\left(A_{1}\right) \cup \ldots \cup N\left(A_{n}\right)$ (for arbitrary sets $A_{1}, \ldots, A_{n}$ ), we may restrict ourselves to the case where $A \subset B_{t / 3}(\mathbf{c}$ ) for some $\mathbf{c} \in \mathbb{R}^{3}$. By the translational invariance of $\lambda$ we may assume $\mathbf{c}=0$.

Let

$$
Y:=\left\{\mathbf{x} \in \mathbb{R}^{3}: x_{3} \geq 0 \text { and } \sqrt{x_{1}^{2}+x_{2}^{2}} \leq t / 3\right\} .
$$

Since $N(A)$ is compact, it can be covered by finitely many rotated $Y$. By the rotational invariance of $\lambda$ it suffices to show that $N:=N(A) \cap Y$ is a null set.

Let $\mathbf{x} \in N$. This implies that $A \subset G:=B_{t}(\mathbf{x})^{c} \cap C$, where

$$
C:=\left\{\mathbf{y} \in \mathbb{R}^{3}:\left|\left(y_{1}, y_{2}\right)-\left(x_{1}, x_{2}\right)\right| \leq \frac{2}{3} t \text { and } y_{3} \leq x_{3}\right\}
$$

Indeed, we have

$$
t=d(\mathbf{x}, A) \leq d(\mathbf{x}, 0)+d(0, A) \leq \sqrt{t^{2} / 9+x_{3}^{2}}+t / 3
$$

which implies $x_{3} \geq \sqrt{3} t / 3>t / 3$. Hence $B_{t / 3}(0) \subset C$, whence $A \subset C$. Plainly, $A \cap B_{t}(\mathbf{x})=\varnothing$.

For $\mathbf{a}=(0,0, \alpha)$ with $\alpha>0$ we have

$$
d(\mathbf{x}+\mathbf{a}, A) \geq d(\mathbf{x}+\mathbf{a}, G)=|\mathbf{x}+\mathbf{a}-\mathbf{y}|=\left(t^{2}+\frac{2}{3} \sqrt{5} \alpha t+\alpha^{2}\right)^{1 / 2}>t,
$$

where $\mathbf{y}:=\mathbf{x}+(2 t / 3,0,-\sqrt{5} t / 3)$, see Figure 2.1. This implies $\mathbf{x}+\mathbf{a} \notin N(A)$, whence $\mathbf{x} \notin N-\mathbf{a}$, and therefore $N \cap(N-\mathbf{a})=\varnothing$. This also shows that $\left(N+\alpha e_{3}\right) \cap\left(N+\alpha^{\prime} e_{3}\right)=$ $\varnothing$ for all $\alpha^{\prime} \neq \alpha$.

But now we have

$$
n \lambda(N)=\lambda\left(\bigcup_{k=1}^{n}\left(N+e_{3} / k\right)\right) \leq \lambda\left(\bigcup_{k=1}^{\infty}\left(N+e_{3} / k\right)\right)<\infty \quad \forall n \in \mathbb{N},
$$

which is only possible if $\lambda(N)=0$. 


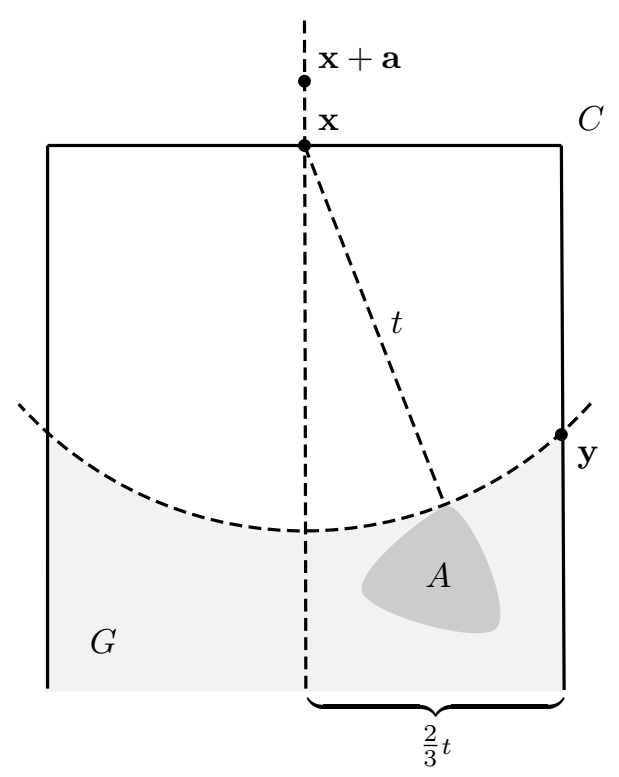

Figure 2.1: The construction of $G$ and $\mathbf{y}$ in the proof of Lemma 2.6

2.7 Lemma. Let $(U, E)$ be a localization or an unsharp localization on a complex separable Hilbert space $\mathscr{H}$ and let $\lambda$ be the Lebesgue measure on $\mathbb{R}^{3}$. For $N \in \mathscr{B}\left(\mathbb{R}^{3}\right)$ we have $E(N)=0$ if and only if $\lambda(N)=0$.

Proof. Put $\mu: \mathscr{B}\left(\mathbb{R}^{3}\right) \rightarrow \mathbb{R}$,

$$
\mu(\Delta):=\sum_{n=1}^{\infty} 2^{-n}\left\langle f_{n}, E(\Delta) f_{n}\right\rangle
$$

where $\left\{f_{1}, f_{2}, \ldots\right\}$ is a countable total set of normalized elements in $\mathscr{H}$. It is easy to see that $\mu$ defines a finite measure on $\mathscr{B}\left(\mathbb{R}^{3}\right)$.

Let $N \in \mathscr{B}\left(\mathbb{R}^{3}\right)$. If $E(N)=0$, then obviously $\mu(N)=0$. If $\mu(N)=0$, then $\left\|\sqrt{E(N)} f_{n}\right\|^{2}=\left\langle f_{n}, E(N) f_{n}\right\rangle=0$ for all $n \in \mathbb{N}$. Hence $E(N) f_{n}=0$ for all $n \in \mathbb{N}$. Therefore, by continuity, $E(N)=0$. Hence, $\mu(N)=0$ if and only if $E(N)=0$. By the covariance property it is $E(N+\mathbf{a})=U((\mathbf{a}, I)) E(N) U((\mathbf{a}, I))^{-1}$ for every $\mathbf{a} \in \mathbb{R}^{3}$. So, $\mu(N)=0 \Longleftrightarrow E(N)=0 \Longleftrightarrow E(N+\mathbf{a})=0 \Longleftrightarrow \mu(N+\mathbf{a})=0$ for every $\mathbf{a} \in \mathbb{R}^{3}$. So $\mu$ is quasi-invariant under translations, and by Lemma G.7 $\mu$ is equivalent to the Lebesgue measure.

2.8 Lemma. Let $S, T \subset \mathbb{R}^{3}$ and let $t \in \mathbb{R}$. Then

(a) $S_{t}=\bigcup_{\mathbf{x} \in S} \overline{B_{|t|}(\mathbf{x})}$ for $t \neq 0$.

(b) $(S \cup T)_{t}=S_{t} \cup T_{t}$.

(c) $S \subset T$ implies $S_{t} \subset T_{t}$. 
(d) $\left(B_{r}(\mathbf{x})\right)_{t}=B_{r+t}(\mathbf{x})$ for all $\mathbf{x} \in \mathbb{R}^{3}$ and $r>0$.

(e) If $S$ is compact then $S_{t}$ is compact and

$$
S_{t}=\left\{\mathbf{z} \in \mathbb{R}^{3}: d(\mathbf{z}, S) \leq|t|\right\}
$$

Proof. The statements (a) and (d) are clear.

(b) and (c) follow from (a) and the fact that $S_{0}=S$.

(e) For $t=0$ there is nothing to show, thus let $t \neq 0$. Since $S$ is bounded, (c) and (d) imply that $S_{t}$ is bounded. For a compact $S$ it is

$$
S_{t}=\left\{\mathbf{z} \in \mathbb{R}^{3}: d(\mathbf{z}, S) \leq|t|\right\}
$$

Indeed, if $\mathbf{y} \in S_{t}$, then there exists an $\mathbf{x} \in S$ such that $\mathbf{y} \in \overline{B_{|t|}(\mathbf{x})}$. Thus $d(\mathbf{y}, S) \leq$ $d(\mathbf{y}, \mathbf{x}) \leq|t|$, hence $\mathbf{y} \in\left\{\mathbf{z} \in \mathbb{R}^{3}: d(\mathbf{z}, S) \leq|t|\right\}$. If $d(\mathbf{y}, S) \leq|t|$ then there exists a sequence $\left(\mathbf{x}_{n}\right)_{n \in \mathbb{N}}$ in $S$ such that $d\left(\mathbf{y}, \mathbf{x}_{n}\right) \stackrel{n \rightarrow \infty}{\longrightarrow} d(\mathbf{y}, S)$. By the compactness of $S$ there is a converging subsequence $\left(\mathbf{x}_{n_{k}}\right)_{k \in \mathbb{N}}$ with limit $\mathbf{x} \in S$. Then $d(\mathbf{y}, \mathbf{x})=$ $\lim _{k \rightarrow \infty} d\left(\mathbf{y}, \mathbf{x}_{n_{k}}\right)=d(\mathbf{y}, S) \leq|t|$, thus $\mathbf{y} \in \overline{B_{|t|}(\mathbf{x})}$, and (a) implies $\mathbf{y} \in S_{t}$.

Since $\mathbf{x} \mapsto d(\mathbf{x}, S)$ is continuous, we see that $S_{t}$ is closed.

2.9 Lemma. Let $A, B$ be orthogonal projections in a Hilbert space $\mathscr{H}$. Then

$$
A \leq B \Longleftrightarrow A=B A .
$$

Proof. Let $A \leq B$. Then for $\psi \in \mathscr{H}$ we have

$$
\begin{aligned}
\|(A-B A) \psi\|^{2} & =\langle(I-B) A \psi,(I-B) A \psi\rangle=\langle A \psi,(I-B) A \psi\rangle \\
& =\langle A \psi, A \psi\rangle-\langle A \psi, B A \psi\rangle \leq\langle A \psi, A \psi\rangle-\langle A \psi, A A \psi\rangle=0 .
\end{aligned}
$$

Hence $A=B A$.

Let $A=B A$. Taking the adjoint of this equation shows that $B A=A B$. Thus $(B-A)^{2}=B-2 B A+A=B-A$, hence $B-A$ is an orthogonal projection, in particular it is positive.

2.10 Corollary. Let $(U, E)$ be a localization on a complex separable Hilbert space $\mathscr{H}$ and let $V$ be a continuous unitary one-parameter group commuting with $U$. Then $(V, U, E)$ is a causal localization if and only if

$$
V(t) E_{c}(\Delta)=E_{c}\left(\Delta_{t}\right) V(t) E_{c}(\Delta) \quad \forall t \in \mathbb{R}, \forall \Delta \in \mathscr{B}\left(\mathbb{R}^{3}\right),
$$

where $E_{c}$ is the completion of $E$.

Proof. Let $t \in \mathbb{R}$ and $\Delta \in \mathscr{B}\left(\mathbb{R}^{3}\right)$. Put $A:=V(t) E_{c}(\Delta) V(-t)$ and $B:=E_{c}\left(\Delta_{t}\right)$. The assertion now follows from Lemma 2.9. 
2.11 Lemma. Let $(U, E)$ be a localization on a complex separable Hilbert space $\mathscr{H}$ and let $V$ be a continuous unitary one-parameter group commuting with $U$. Then $(V, U, E)$ is a causal localization if and only if

$$
V(t) E(\Delta)=E\left(\Delta_{t}\right) V(t) E(\Delta) \quad \forall t \in \mathbb{R}
$$

holds for every open ball $\Delta$ centered at the origin.

Proof. The "only if" part of the Lemma is trivial. To prove the "if" part of the Lemma we may assume that $t>0$, since the case $t=0$ is trivial and since $\Delta_{-t}=\Delta_{t}$.

By the covariance property we see that all open balls satisfy 2.1 . Let $\mathscr{C}$ be the collection of all $A \in \mathscr{B}\left(\mathbb{R}^{3}\right)$ which satisfy $A_{t} \in \mathscr{B}\left(\mathbb{R}^{3}\right)$ and (2.1).

(a) If $A, B \in \mathscr{C}$ then $A \cup B \in \mathscr{C}$ : Clearly $(A \cup B)_{t}=A_{t} \cup B_{t} \in \mathscr{B}\left(\mathbb{R}^{3}\right)$. Using

$$
E(A \cup B)=E(A)+E(B)-E(A) E(B)
$$

and $E(A) E(B)=E(B) E(A)$ we find, omitting the $E$ and the argument of $V$,

$$
\begin{aligned}
(A \cup B)_{t} V(A \cup B)= & \left(A_{t}+B_{t}-A_{t} B_{t}\right) V(A+B-A B) \\
= & A_{t} V A+A_{t} V B-A_{t} V A B+B_{t} V A+B_{t} V B-B_{t} V A B \\
& -A_{t} B_{t} V A-A_{t} B_{t} V B+A_{t} B_{t} V A B \\
= & V A+A_{t} V B-V A B+B_{t} V A+V B-V A B \\
& -B_{t} V A-A_{t} V B+V A B \\
= & V A+V B-V A B=V(A \cup B) .
\end{aligned}
$$

(b) By induction we obtain: If $A_{1}, A_{2}, \ldots, A_{n} \in \mathscr{C}$ then $\cup_{i=1}^{n} A_{i} \in \mathscr{C}$.

(c) Every compact set is in $\mathscr{C}$ : Let $K \subset \mathbb{R}^{3}$ be compact. We will construct a sequence $\left(U_{n}\right)_{n \in \mathbb{N}}$ of open sets, each being a finite union of open balls, such that

$$
U_{n+1} \subset U_{n}, \quad K=\cap_{n=1}^{\infty} U_{n}, \quad K_{t}=\cap_{n=1}^{\infty}\left(U_{n}\right)_{t} .
$$

Let $A_{1} \subset K$ be a finite set such that

$$
K \subset U_{1}:=\bigcup_{\mathbf{a} \in A_{1}} B_{1}(\mathbf{a})
$$

Put $\alpha_{1}:=d\left(K, U_{1}^{c}\right) / 2$, where $d(X, Y):=\inf _{\mathbf{x} \in X} d(\mathbf{x}, Y)$. Note that $U_{1}^{c}$ is closed and $K \cap U_{1}^{c}=\varnothing$. Thus by the compactness of $K$ we have $0<\alpha_{1}$. It is also clear that

$$
d\left(K, U_{1}^{c}\right)=\inf _{\mathbf{x} \in K} d\left(\mathbf{x}, U_{1}^{c}\right) \leq \inf _{\mathbf{a} \in A_{1}} d\left(\mathbf{a}, U_{1}^{c}\right) \leq 1
$$

hence $\alpha_{1} \leq 1 / 2$.

If $U_{1}, \ldots, U_{n}$ and $\alpha_{1}, \ldots, \alpha_{n}$ have been chosen, let $A_{n+1}$ be a finite subset of $K$ such that

$$
K \subset U_{n+1}:=\bigcup_{\mathbf{a} \in A_{n+1}} B_{\alpha_{n}}(\mathbf{a})
$$


Put $\alpha_{n+1}:=d\left(K, U_{n+1}^{c}\right) / 2$. Clearly $0<\alpha_{n+1} \leq \alpha_{n} / 2$. Since $d\left(K, U_{n}^{c}\right)=2 \alpha_{n}$, we have

$$
\bigcup_{\mathbf{x} \in K} B_{\alpha_{n}}(\mathbf{x}) \subset U_{n}
$$

Hence $U_{n+1} \subset U_{n}$.

We show that $K_{t}=\cap_{n=1}^{\infty}\left(U_{n}\right)_{t} . K \subset U_{n}$ implies $K_{t} \subset\left(U_{n}\right)_{t}$ for all $n \in \mathbb{N}$ and thus $K_{t} \subset \cap_{n=1}^{\infty}\left(U_{n}\right)_{t}$. Let $\mathbf{y} \in \cap_{n=1}^{\infty}\left(U_{n}\right)_{t}$. Then $\mathbf{y} \in\left(U_{n+1}\right)_{t}=\bigcup_{\mathbf{a} \in A_{n+1}} B_{t+\alpha_{n}}(\mathbf{a})$ for all $n \in \mathbb{N}$. Thus for each $n \in \mathbb{N}$ exists an $\mathbf{x}_{n} \in K$ such that $\mathbf{y} \in B_{t+\alpha_{n}}\left(\mathbf{x}_{n}\right)$. By the compactness of $K$ there exists a subsequence $\left(\mathbf{x}_{n(k)}\right)_{k \in \mathbb{N}}$ converging to some $\mathbf{x} \in K$. Then

$$
|\mathbf{y}-\mathbf{x}| \leq\left|\mathbf{y}-\mathbf{x}_{n(k)}\right|+\left|\mathbf{x}_{n(k)}-\mathbf{x}\right| \leq t+2^{-n(k)}+\left|\mathbf{x}_{n(k)}-\mathbf{x}\right| \stackrel{k \rightarrow \infty}{\longrightarrow} t
$$

implies that $\mathbf{y} \in K_{t}$. The same arguments imply that $K=\cap_{n=1}^{\infty} U_{n}$.

Since $\Delta \mapsto E_{\phi}(\Delta):=\langle\phi, E(\Delta) \phi\rangle$ is a finite Borel measure for every $\phi \in \mathscr{H}$, we have

$$
\begin{aligned}
\langle\psi, V(t) E(K) V(-t) \psi\rangle & =\lim _{n \rightarrow \infty}\left\langle\psi, V(t) E\left(U_{n}\right) V(-t) \psi\right\rangle \\
& \leq \lim _{n \rightarrow \infty}\left\langle\psi, E\left(\left(U_{n}\right)_{t}\right) \psi\right\rangle=\left\langle\psi, E\left(K_{t}\right) \psi\right\rangle,
\end{aligned}
$$

for every $\psi \in \mathscr{H}$. Note that $K_{t} \in \mathscr{B}\left(\mathbb{R}^{3}\right)$, since it is compact. This proves (c).

Finally, let $B$ be a Borel set and $\psi \in \mathscr{H}$. Because $E_{\phi}$ is regular for every $\phi \in \mathscr{H}$ (see, e.g., [[Rud70] Theorem 2.18]) we have

$$
\begin{aligned}
\langle\psi, V(t) E(B) V(-t) \psi\rangle & =\sup \{\langle\psi, V(t) E(K) V(-t) \psi\rangle: K \subset B, K \text { compact }\} \\
& \leq \sup \left\{\left\langle\psi, E\left(K_{t}\right) \psi\right\rangle: K \subset B, K \text { compact }\right\} \\
& \leq\langle\psi, E(C(B, t)) \psi\rangle
\end{aligned}
$$

since $K_{t} \subset B_{t} \subset C(B, t)$. By Discussion 2.5 and Lemma 2.6 we have $E(C(B, t))=$ $E_{c}\left(B_{t}\right)$, where $E_{c}$ denotes the completion of $E$. For Borel sets it is $E_{c}(B)=E(B)$, so we have

$$
\left\langle\psi, V(t) E_{c}(B) V(-t) \psi\right\rangle \leq\left\langle\psi, E_{c}\left(B_{t}\right) \psi\right\rangle
$$

This completes the proof.

2.12 Discussion. In order to study causal localizations, Castrigiano introduced in Cas84 causal transformations (see Appendix F for a brief summary), which are more general than causal time evolutions. But if $V$ is a causal time evolution, then for every $t \in \mathbb{R}, V(t)$ is a causal transformation. So the necessary conditions developed in Cas84 for a causal transformation readily apply to causal time evolutions. With the help of the last Lemma and the results of Part II we can now adapt the sufficient conditions.

2.13 Proposition. (cf. Cas84 Lemma 2). Let $(U, E)$ be the coordinate representation of a finite localization and let $V$ be a unitary continuous one-parameter group. Then $(V, U, E)$ is a causal localization if and only if 
(a) For each $t \in \mathbb{R}$ there exists an entire matrix-valued function $\Phi_{t}: \mathbb{C}^{3} \rightarrow L\left(\underset{j \in \Omega}{\oplus} \nu_{j} \mathbb{C}^{2 j+1}\right)$ such that in the momentum space representation

$$
\hat{V}(t)[f]=\left[\left.\Phi_{t}\right|_{\mathbb{R}^{3}} f\right] \quad \forall[f] \in \mathscr{H} .
$$

(b) For each $t \in \mathbb{R}$ there exists a constant $C_{t}>0$ such that

$$
\left\|\Phi_{t}(\mathbf{z})\right\| \leq C_{t} e^{|t||\mathbf{z}|} \quad \forall \mathbf{z} \in \mathbb{C}^{3},
$$

where $|\mathbf{z}|:=\sqrt{\left|z_{1}\right|^{2}+\left|z_{2}\right|^{2}+\left|z_{3}\right|^{2}}$.

(c) For every $t \in \mathbb{R}$ we have

$$
\Phi_{t}\left(|\mathbf{p}| e_{3}\right)=D\left(B(\mathbf{p})^{-1}\right) \Phi_{t}(\mathbf{p}) D(B(\mathbf{p})) \quad \forall \mathbf{p} \in \mathbb{R}^{3},
$$

where $D(B):=\oplus_{j \in \Omega} \nu_{j} D^{(j)}(B)$.

(d) For every $\mathbf{p} \in \mathbb{R}^{3}$ it is

$$
\left\langle k, \kappa, r\left|\Phi_{t}\left(|\mathbf{p}| e_{3}\right)\right| j, \iota, s\right\rangle=0 \quad \forall r \neq s .
$$

Proof. The "if" part of the Proposition: Put $M: \mathbb{R}_{\geq 0} \rightarrow L\left(\oplus_{j \in \Omega} \nu_{j} \mathbb{C}^{2 j+1}\right)$,

$$
M(\rho):=\Phi_{t}\left(\rho e_{3}\right) .
$$

Then (c) shows that in the helicity representation $V^{h}(t)[f]=[M(|\cdot|) f]$. Lemma 1.13 and (d) imply that $V$ commutes with $U$.

Let $t \in \mathbb{R}$. From (a) and Lemma 1.11 we have $\left\|\left.\Phi_{t}\right|_{\mathbb{R}^{3}}(\cdot)\right\|=1$ a.e. By continuity this must hold everywhere. Then by (b) and Theorem 10.15 (b) we find

$$
V(t) E(\Delta)=E\left(\Delta_{t}\right) V(t) E(\Delta)
$$

for every open ball $\Delta$ centered at the origin. Lemma 2.11 shows that $(V, U, E)$ is a causal localization.

It follows the "only if" part of the Proposition. Since $V$ commutes with $U$, there exists for each $t \in \mathbb{R}$ a measurable bounded matrix-valued function $A_{t}: \mathbb{R}^{3} \rightarrow$ $L\left(\oplus_{j \in \Omega} \nu_{j} \mathbb{C}^{2 j+1}\right)$ such that in the momentum space representation

$$
\hat{V}(t)[f]=\left[A_{t} f\right] \quad \forall[f] \in \mathscr{H} .
$$

Since $|\operatorname{Im} \mathbf{z}| \leq|\mathbf{z}|$, (a) and (b) follow from Theorem 10.15 (a).

Let $t \in \mathbb{R}$ and let $B \in S U(2)$. Then $[V(t), U(B)]=0$ implies

$$
\Phi_{t}(\mathbf{p}) D(B)=D(B) \Phi_{t}\left(B^{-1} \cdot \mathbf{p}\right) \quad \text { a.e. }
$$

By continuity this must hold everywhere. Hence

$$
D(B(\mathbf{p}))^{-1} \Phi_{t}(\mathbf{p}) D(B(\mathbf{p}))=\Phi_{t}\left(B(\mathbf{p})^{-1} \cdot \mathbf{p}\right)=\Phi_{t}\left(|\mathbf{p}| e_{3}\right) .
$$

This shows (c).

In the helicity representation we have $V^{h}(t)[f]=\left[\Phi_{t}\left(|\cdot| e_{3}\right) f\right]$, and Lemma 1.13 implies that (d) holds almost everywhere. By the continuity of $\Phi_{t}$ condition (d) holds everywhere. 
2.14 Theorem. (cf. Theorem of [Cas84]). Let $(U, E)$ be the coordinate representation of a finite localization and let $V$ be a unitary continuous one-parameter group. Then $(V, U, E)$ is a causal localization if and only if

(a) For each $t \in \mathbb{R}$ there exists an entire matrix-valued function $\Psi_{t}: \mathbb{C} \rightarrow L\left(\underset{j \in \Omega}{\oplus} \nu_{j} \mathbb{C}^{2 j+1}\right)$ such that in the helicity representation

$$
V^{h}(t)[f]=\left[\Psi_{t}(|\cdot|) f\right] \quad \forall[f] \in \mathscr{H} .
$$

(b) For each $t \in \mathbb{R}$ there exists a constant $C_{t}>0$ such that

$$
\left\|\Psi_{t}(z)\right\| \leq C_{t} e^{|t||z|} \quad \forall z \in \mathbb{C} .
$$

(c) For each $t \in \mathbb{R}$ there are entire functions $f_{t, \kappa, l}^{(k, j, l)}: \mathbb{C} \rightarrow \mathbb{C}$ such that

$$
\left\langle k, \kappa, r\left|\Psi_{t}(z)\right| j, \iota, s\right\rangle=\delta_{r s} \sum_{l=|k-j|}^{k+j}(-1)^{j-s} \sqrt{2 l+1}\left(\begin{array}{rrr}
j & k & l \\
-s & s & 0
\end{array}\right) z^{l} f_{t, \kappa, l}^{(k, j, l)}\left(z^{2}\right) \quad \forall z \in \mathbb{C} .
$$

Proof. The "if" part of the Theorem: Since $V(0)=I$, the case $t=0$ is trivial. Let $t \neq 0$. Put $A_{t}: \mathbb{R}^{3} \rightarrow L\left(\underset{j \in \Omega}{\oplus} \nu_{j} \mathbb{C}^{2 j+1}\right)$,

$$
A_{t}(\mathbf{p}):=D(B(\mathbf{p})) \Psi_{t}(|\mathbf{p}|) D\left(B(\mathbf{p})^{-1}\right),
$$

where $D(B):=\oplus_{j \in \Omega} \nu_{j} D^{(j)}(B)$. Thus in the momentum space representation it is

$$
\hat{V}(t)[f]=\left[A_{t} f\right] \quad \forall[f] \in \mathscr{H} .
$$

For fixed $j$ and $k$ put $\varphi_{r s}^{(l)}: \mathbb{R}^{3} \rightarrow \mathbb{C}$,

$$
\varphi_{r s}^{(l)}(\mathbf{p}):=\sum_{v, u} D^{(k)}(B(\mathbf{p}))_{r v}(-1)^{j-u} \sqrt{2 l+1}\left(\begin{array}{rrr}
j & k & l \\
-u & v & 0
\end{array}\right)|\mathbf{p}|^{l} D^{(j)}\left(B(\mathbf{p})^{-1}\right)_{u s} .
$$

Then, since $\langle k, \kappa, r|D(B(\mathbf{p}))| l, \lambda, v\rangle=\delta_{k l} \delta_{\kappa \lambda} D^{(k)}(B(\mathbf{p}))_{r v}$ and

$$
\left(\begin{array}{rrr}
j & k & l \\
-u & v & 0
\end{array}\right)=0 \quad \forall v \neq u
$$

condition (c) implies

$$
\left\langle k, \kappa, r\left|A_{t}(\mathbf{p})\right| j, \iota, s\right\rangle=\sum_{l} \varphi_{r s}^{(l)}(\mathbf{p}) f_{t, \kappa, \iota}^{(k, j, l)}\left(|\mathbf{p}|^{2}\right) .
$$

By [Cas84] (19) fol.] the $\varphi_{r s}^{(l)}$ are polynomials. Thus

$$
\mathbf{z} \mapsto\left\langle k, \kappa, r\left|\Phi_{t}(\mathbf{z})\right| j, \iota, s\right\rangle:=\sum_{l} \varphi_{r s}^{(l)}(\mathbf{z}) f_{t, \kappa, \iota}^{(k, j, l)}\left(z_{1}^{2}+z_{2}^{2}+z_{3}^{2}\right),
$$


defines an entire extension for $A_{t}$. Using the orthogonality relations for the Wigner $3 j$ symbols it is easy to see that (c) implies

$$
z^{l} f_{t, \kappa, l}^{(k, j)}\left(z^{2}\right)=\sum_{s}(-1)^{j-s} \sqrt{2 l+1}\left(\begin{array}{rrr}
j & k & l \\
-s & s & 0
\end{array}\right)\left\langle k, \kappa, s\left|\Psi_{t}(z)\right| j, \iota, s\right\rangle .
$$

By (b) we have

$$
\left|f_{t, \kappa, l}^{(k, j, l)}\left(z^{2}\right)\right| \leq C_{t} e^{|t||z|} \quad \forall z \in \mathbb{C},
$$

for some constant $C_{t}>0$. This implies (using $|z|=\left|z^{2}\right|^{1 / 2}$ )

$$
\left|f_{t, \kappa, \iota}^{(k, j, l)}\left(z_{1}^{2}+z_{2}^{2}+z_{3}^{2}\right)\right| \leq C_{t} e^{|t|\left|z_{1}^{2}+z_{2}^{2}+z_{3}^{2}\right|^{1 / 2}} \leq C e^{|t||\mathbf{z}|} \quad \forall \mathbf{z} \in \mathbb{C}^{3} .
$$

Let $\varepsilon>0$. Then, since the $\varphi_{r s}^{(l)}$ are polynomials, there exists a constant $C_{\varepsilon}>0$ such that

$$
\left|\varphi_{r s}^{(l)}(\mathbf{z})\right| \leq C_{\varepsilon} e^{\varepsilon|\mathbf{z}|} \quad \forall \mathbf{z} \in \mathbb{C}^{3} .
$$

Hence

$$
\left|\left\langle k, \kappa, r\left|\Phi_{t}(\mathbf{z})\right| j, \iota, s\right\rangle\right| \leq C_{t, \varepsilon}^{\prime} e^{(|t|+\varepsilon)|\mathbf{z}|} \quad \forall \mathbf{z} \in \mathbb{C}^{3},
$$

for some constant $C_{t, \varepsilon}^{\prime}>0$. The estimate $\left\|\left.\Phi_{t}\right|_{\mathbb{R}^{3}}(\cdot)\right\| \leq\|\hat{V}(t)\|=1$ holds a.e. (Lemma 1.11), and by the continuity of $\Phi_{t}$ this holds everywhere. Using the equivalence of the operator norm and the entry-wise norm on $L\left(\oplus_{j \in \Omega} \nu_{j} \mathbb{C}^{2 j+1}\right)$ and Corollary 10.17, we find $\left\|\Phi_{t}(\mathbf{z})\right\| \leq e^{(|t|+\varepsilon)|\mathbf{z}|}$ for all $\mathbf{z} \in \mathbb{C}^{3}$. Since $\varepsilon$ was arbitrary we have

$$
\left\|\Phi_{t}(\mathbf{z})\right\| \leq e^{|t||\mathbf{z}|} \quad \forall \mathbf{z} \in \mathbb{C}^{3} .
$$

This proves conditions (a) and (b) of Proposition 2.13

Since $B\left(|\mathbf{p}| e_{3}\right)=I$, we have $A_{t}\left(|\mathbf{p}| e_{3}\right)=\Psi_{t}(|\mathbf{p}|)$ for all $\mathbf{p} \in \mathbb{R}^{3}$. This implies

$$
A_{t}\left(|\mathbf{p}| e_{3}\right)=D\left(B(\mathbf{p})^{-1}\right) A_{t}(\mathbf{p}) D(B(\mathbf{p})) \quad \forall \mathbf{p} \in \mathbb{R}^{3},
$$

and, by means of (c),

$$
\left\langle k, \kappa, r\left|A_{t}\left(|\mathbf{p}| e_{3}\right)\right| j, \iota, s\right\rangle=0 \quad \forall r \neq s .
$$

This proves conditions (c) and (d) of Proposition 2.13

The "only if" part of the Theorem: Let $\Phi_{t}: \mathbb{C}^{3} \rightarrow L\left(\oplus_{j \in \Omega} \nu_{j} \mathbb{C}^{2 j+1}\right)$ be the function given by Proposition 2.13. Then $\Psi_{t}: \mathbb{C} \rightarrow L\left(\oplus_{j \in \Omega} \nu_{j} \mathbb{C}^{2 j+1}\right), \Psi_{t}(z):=\Phi_{t}\left(z e_{3}\right)$ satisfies (a) and (b).

Condition (c) follows from the main Theorem in [Cas84, since $V(t)$ is a causal transformation for every $t \in \mathbb{R}$.

2.15 Remark. Using the orthogonality relations for the Wigner $3 j$ symbol (see Appendix E), we see that

$$
\langle k, \kappa, r|\Psi(z)| j, \iota, s\rangle=\delta_{r s} \sum_{l=|k-j|}^{k+j}(-1)^{j-s} \sqrt{2 l+1}\left(\begin{array}{rrr}
j & k & l \\
-s & s & 0
\end{array}\right) z^{l} f_{\kappa, \iota}^{(k, j, l)}\left(z^{2}\right)
$$


if and only if

$$
z^{l} f_{\kappa, \iota}^{(k, j, l)}\left(z^{2}\right)=\sum_{s \in[k] \cap[j]}(-1)^{j-s} \sqrt{2 l+1}\left(\begin{array}{rrr}
j & k & l \\
-s & s & 0
\end{array}\right)\langle k, \kappa, s|\Psi(z)| j, \iota, s\rangle .
$$

This, however, does not imply that condition (c) of Theorem 2.14 is trivial, since in general $g_{\kappa, L}^{(k, j, l)}: \mathbb{R}_{>0} \rightarrow \mathbb{C}$,

$$
g_{\kappa, \iota}^{(k, j, l)}(\rho):=\rho^{-l / 2} \sum_{s \in[k] \cap[j]}(-1)^{j-s} \sqrt{2 l+1}\left(\begin{array}{rrr}
j & k & l \\
-s & s & 0
\end{array}\right)\left\langle k, \kappa, s\left|\Psi\left(\rho^{1 / 2}\right)\right| j, \iota, s\right\rangle .
$$

has no entire extension.

2.16 Discussion. So far, the fact that $V$ is a homomorphism has played no role. The consequences of this property are studied in section 11. In brief, the situation is as follows: We have seen that in the helicity representation $V^{h}(t)[f]=\left[\Psi_{t}^{h}(|\cdot|) f\right]$, where $\Psi_{t}^{h}: \mathbb{C} \rightarrow L\left(\oplus_{j \in \Omega} \nu_{j} \mathbb{C}^{2 j+1}\right)$ is an exponentially bounded entire matrix-valued function. Then Stone's Theorem leads us to the conjecture that $\Psi_{t}^{h}=e^{i t h(\cdot)}$ for some entire matrix-valued function $h$ such that $h(\rho)$ is self-adjoint for all $\rho>0$. That this is indeed the case is the subject of Theorem 11.10. In Theorem 12.6 it is shown that the exponential boundedness of $e^{i t h(\cdot)}$ implies that $h$ is linear. In the next Theorem, which is a generalization of [[Cas84] Lemma 7 fol.], we apply these results.

2.17 Theorem. Let $(U, E)$ be the coordinate representation of a finite localization and let $V$ be a continuous unitary one-parameter group. If $(V, U, E)$ is a causal localization, then there exist self-adjoint matrices $M, N \in L\left(\oplus_{j \in \Omega} \nu_{j} \mathbb{C}^{2 j+1}\right)$ such that in the helicity representation

$$
V^{h}(t)[f]=\left[e^{i t(M+|\cdot| N)} f\right] \quad \forall t \in \mathbb{R}, \quad \forall[f] \in \mathscr{H},
$$

and

$$
\langle k, \kappa, r|N| j, \iota, s\rangle=0 \quad \forall r \neq s, \quad\langle k, \kappa, r|M| j, \iota, s\rangle=c_{\kappa \iota}^{(k)} \delta_{r s} \delta_{k j},
$$

for some constants $c_{\kappa i}^{(k)} \in \mathbb{C}$. Moreover, the operator $T$ defined as $T^{h}[f]:=[M f]$ commutes with $(U, E)$, and $T=\hat{T}=T^{h}$.

Proof. Theorem 2.14 and Theorem 11.10 imply that in the helicity representation

$$
V^{h}(t)[f]=\left[e^{i t h(|\cdot|)} f\right],
$$

where $h: \mathbb{C} \rightarrow L\left(\oplus_{j \in \Omega} \nu_{j} \mathbb{C}^{2 j+1}\right)$ is an entire matrix-valued function such that $h(\rho)$ is self-adjoint for all $\rho \in \mathbb{R}$. Moreover, for each $t \in \mathbb{R}$ we have

$$
\left\|e^{i t h(z)}\right\| \leq C_{t} e^{|t||z|} \quad \forall z \in \mathbb{C},
$$

where $C_{t}>0$ is a constant depending on $t$. In particular, $\delta\left(e^{i t h(\cdot)}\right) \leq|t|$. From Theorem 12.6 we have self-adjoint matrices $M, N \in L\left(\oplus_{j \in \Omega} \nu_{j} \mathbb{C}^{2 j+1}\right)$ such that $h(z)=M+z N$ for all $z \in \mathbb{C}$. 
Let $t \in \mathbb{R}$ and put $B_{\phi}:=\left(\begin{array}{cc}e^{i \phi} & 0 \\ 0 & e^{-i \phi}\end{array}\right)$. By Lemma 1.9 it is $R\left(\mathbf{p}, B_{\phi}\right)=B_{\phi}$ for $\mathbf{p} \neq-|\mathbf{p}| e_{3}$. Since $V(t)$ commutes with $U\left(0, B_{\phi}\right)$, there exists for each $\phi$ a null set $S_{\phi}$ such that in the helicity representation

$$
e^{i t h(|\mathbf{p}|)} D\left(B_{\phi}\right)=D\left(B_{\phi}\right) e^{i t h(|\mathbf{p}|)} \quad \forall \mathbf{p} \in \mathbb{R}^{3} \backslash S_{\phi},
$$

where $D(B):=\oplus_{j \in \Omega} \nu_{j} D^{(j)}(B)$, i.e.

$$
\left\langle k, \kappa, r\left|D\left(B_{\phi}\right)\right| l, \lambda, u\right\rangle=\delta_{k l} \delta_{\kappa \lambda} \delta_{r u} e^{i \phi 2 r} .
$$

By continuity, Eq. (2.3) must hold for all $\mathbf{p} \in \mathbb{R}^{3}$ and then also for all $t \in \mathbb{R}$. The derivative with respect to $t$ of the equation at $t=0$ gives

$$
h(|\mathbf{p}|) D\left(B_{\phi}\right)=D\left(B_{\phi}\right) h(|\mathbf{p}|) \quad \forall \mathbf{p} \in \mathbb{R}^{3}, \forall \phi \in \mathbb{R} .
$$

Thus for $\mathbf{p}=0$ we have $M D\left(B_{\phi}\right)=D\left(B_{\phi}\right) M$ and then for $\mathbf{p} \neq 0$ this implies $N D\left(B_{\phi}\right)=D\left(B_{\phi}\right) N$. Hence $\langle k, \kappa, r|N| j, \iota, s\rangle=0$ for all $r \neq s$.

By Theorem 2.14 (c) it is

$$
\left\langle k, \kappa, r\left|e^{i t(M+z N)}\right| j, \iota, s\right\rangle=\delta_{r s} \sum_{l=|j-k|}^{j+k} D_{s, l}^{(k, j)} z^{l}\left(f_{t, j k l}\left(z^{2}\right)\right)_{\kappa \iota} \quad \forall z \in \mathbb{C},
$$

where $D_{s, l}^{(k, j)}:=(-1)^{j-s} \sqrt{2 l+1}\left(\begin{array}{ccc}j & k & l \\ -s & s & 0\end{array}\right)$. Thus for $z=0$ we have

$$
\left\langle k, \kappa, r\left|e^{i t M}\right| j, \iota, s\right\rangle=\delta_{r s} \delta_{k j} D_{s, 0}^{(k, j)}\left(f_{t, j k 0}(0)\right)_{\kappa \iota} \quad \forall t \in \mathbb{R}
$$

which implies

$$
\langle k, \kappa, r|M| j, \iota, s\rangle=\delta_{r s} \delta_{k j} D_{s, 0}^{(k, k)}\left(f_{k k}^{\prime}\right)_{\kappa \iota}
$$

where $f_{j k}^{\prime}:=-i \partial_{t} f_{0, j k 0}(0)$. Note that $D_{s, 0}^{(k, k)}=(-1)^{2 k}(2 k+1)^{-1 / 2}$ (see E.1) is independent of $s$. Using Lemma 1.14 completes the proof.

2.18 Note. We like to stress that the above conditions are not sufficient for $(V, U, E)$ to be a causal localization, since $D(B(\cdot))(M+|\cdot| N) D\left(B(\cdot)^{-1}\right)$ in general has no analytic extension to $\mathbb{C}^{3}$ (e.g. $M=0, N=I$ ). However, we will show in Theorem 5.10 that every finite massive relativistic extendable causal localization is a direct sum of Dirac- and Dirac tensor-systems. The most simple non-relativistic systems are studied in Theorem 7.1 . 


\section{Finite Massive Representations of the Poincaré Group}

3.1. The massive representations of $\operatorname{ISL}(2, \mathbb{C})$. In this subsection we recall some well-known facts [adapted from [Sch70 and [Var07] about the universal covering group $I S L(2, \mathbb{C})$ of the Poincaré groun? It consists of elements $(a, A)$, where $a \in \mathbb{R}^{4}, A \in$ $S L(2, \mathbb{C})$, and the group law is given by

$$
(a, A)\left(a^{\prime}, A^{\prime}\right):=\left(a+A a^{\prime}, A A^{\prime}\right),
$$

where $A a:=\Lambda(A) a$ and $\Lambda: S L(2, \mathbb{C}) \rightarrow L_{+}^{\uparrow}$ is the covering homomorphism from $S L(2, \mathbb{C})$ onto the proper orthochronous Lorentz group $L_{+}^{\uparrow}$ (see Appendix $B$ ). The Minkowski product of $p, q \in \mathbb{R}^{4}$ will be written as $\langle p, G q\rangle$, where

$$
G:=\operatorname{diag}(1,-1,-1,-1) .
$$

The irreducible strongly continuous unitary representations of $I S L(2, \mathbb{C})$ corresponding to the Orbits $X^{(\mu, \eta)}:=\left\{p \in \mathbb{R}^{4}:\langle p, G p\rangle=\mu^{2}, \eta p_{0}>0\right\}$ of mass $\mu>0$ and sign of energy $\eta \in\{-1,1\}$ are given by

$$
\begin{aligned}
U^{(\mu, \eta, j)}(a, A) & : L^{2}\left(X^{(\mu, \eta)}, \mathbb{C}^{2 j+1}, \alpha_{\mu}^{\eta}\right) \rightarrow L^{2}\left(X^{(\mu, \eta)}, \mathbb{C}^{2 j+1}, \alpha_{\mu}^{\eta}\right), \\
U^{(\mu, \eta, j)}(a, A)[F] & :=\left[e^{i<\cdot G a\rangle} D^{(j)}\left(R^{(\mu, \eta)}(\cdot, A)\right) F\left(A^{-1} \cdot\right)\right], \\
R^{(\mu, \eta)}(\cdot, A) & :=A^{(\mu, \eta)}(\cdot)^{-1} A A^{(\mu, \eta)}\left(A^{-1} \cdot\right),
\end{aligned}
$$

where $\alpha_{\mu}^{\eta}$ is the invariant measure given by

$$
\int_{X^{(\mu, \eta)}} F d \alpha_{\mu}^{\eta}:=\int_{\mathbb{R}^{3}} \frac{F\left(\eta\left(\mu^{2}+\mathbf{p}^{2}\right)^{1 / 2}, \mathbf{p}\right)}{2\left(\mu^{2}+\mathbf{p}^{2}\right)^{1 / 2}} d \mathbf{p}
$$

and $A^{(\mu, \eta)}: X^{(\mu, \eta)} \rightarrow S L(2, \mathbb{C})$ is a measurable function satisfying

$$
A^{(\mu, \eta)}(p) \mu \eta e_{0}=p
$$

for all $p \in X^{(\mu, \eta)}$. This implies that $R^{(\mu, \eta)}(p, A) e_{0}=e_{0}$ for all $p \in X^{(\mu, \eta)}$, hence $R^{(\mu, \eta)}(p, A) \in S U(2)$. The orbits $X^{(\mu, \eta)}$ can be parameterized by $p^{\eta}: \mathbb{R}^{3} \rightarrow X^{(\mu, \eta)}$,

$$
p^{\eta}(\mathbf{p})=\left(\begin{array}{c}
\eta \epsilon(\mathbf{p}) \\
\mathbf{p}
\end{array}\right), \quad \epsilon(\mathbf{p}):=\sqrt{\mu^{2}+\mathbf{p}^{2}} .
$$

An explicit realization of $A^{(\mu, \eta)}$ is the so-called helicity section:

$$
A^{(\mu, \eta)}(p):=B(\eta \mathbf{p}) A(v), \quad A(v):=\left(\begin{array}{cc}
e^{v / 2} & 0 \\
0 & e^{-v / 2}
\end{array}\right), \quad \mathbf{p}:=\sum_{i=1}^{3} p_{i} \mathbf{e}_{i},
$$

\footnotetext{
${ }^{3}$ Since the Poincaré group is represented projectively on physical states, it is more convenient to use its universal covering group (see, e.g., [WWei95] Sec. 2.7] for details).
} 
where $B: \mathbb{R}^{3} \rightarrow S U(2)$ is given by Eq. (1.2) and $v$ is the non-negative solution of $\cosh (v)=\mu^{-1} \epsilon(\mathbf{p})$. Because $A(v) e_{0}=\left(\cosh v, \sinh v \mathbf{e}_{3}\right)$ and $B(\eta \mathbf{p}) \mathbf{e}_{3}=\eta \mathbf{p} /|\mathbf{p}|$ it is easy to see that the helicity section satisfies Eq. (3.2).

In the following Lemma we will describe a unitarily equivalent representation which has two convenient properties: (i) it is an extension of $U_{D^{(j)}}$, and (ii) its NewtonWigner localization (see Definition 3.5) is the canonical projection-valued measure, cf. [[Mut84] Eq. (2.2) and fol.]. Before we state the Lemma some definitions are in order.

3.2 Definitions. For every $\left(p_{0}, \mathbf{p}\right) \in \mathbb{R}^{4}$ we define

$$
\left(p_{0}, \mathbf{p}\right)^{\downarrow}:=\mathbf{p} .
$$

The canonical cross-section is the map $Q^{(\mu, \eta)}: X^{(\mu, \eta)} \rightarrow S L(2, \mathbb{C})$,

$$
Q^{(\mu, \eta)}(p):=B(\eta \mathbf{p}) A(v) B(\eta \mathbf{p})^{-1},
$$

where $v$ is the non-negative solution of $\cosh (v)=\mu^{-1} \epsilon(\mathbf{p})$.

3.3 Lemma. The representation $U^{(\mu, \eta, j)}$ given in 3.1 is unitarily equivalent to the representation $\hat{W}^{(\mu, \eta, j)}: \operatorname{ISL}(2, \mathbb{C}) \rightarrow L\left(L^{2}\left(\mathbb{R}^{3}, \mathbb{C}^{2 j+1}\right)\right)$,

$$
\left(\hat{W}^{(\mu, \eta, j)}(a, A) f\right)(\mathbf{p}):=\left(\frac{\epsilon\left(\mathbf{q}^{\eta}\right)}{\epsilon(\mathbf{p})}\right)^{1 / 2} e^{i\left\langle p^{\eta}, G a\right\rangle} D^{(j)}\left(Q^{(\mu, \eta)}\left(p^{\eta}\right)^{-1} A Q^{(\mu, \eta)}\left(q^{\eta}\right)\right) f\left(\mathbf{q}^{\eta}\right),
$$

where $q^{\eta}:=A^{-1} p^{\eta}$ and $\mathbf{q}^{\eta}:=\left(q^{\eta}\right)^{\downarrow}$. Moreover, we have $\left.\hat{W}^{(\mu, \eta, j)}\right|_{I S U(2)}=\hat{U}_{D^{(j)}}$.

Proof. First we transform from $L^{2}\left(X^{(\mu, \eta)}, \mathbb{C}^{2 j+1}\right)$ to $L^{2}\left(\mathbb{R}^{3}, \mathbb{C}^{2 j+1}\right)$ by

$$
(S F)(\mathbf{p}):=(2 \epsilon(\mathbf{p}))^{-1 / 2} F\left(p^{\eta}\right), \quad\left(S^{-1} f\right)(p):=\left(2 \epsilon\left(p^{\downarrow}\right)\right)^{1 / 2} f\left(p^{\downarrow}\right) .
$$

The factor $(2 \epsilon(\mathbf{p}))^{-1 / 2}$ ensures that $S$ is unitary. We have

$$
\left(S U^{(\mu, \eta, j)}(a, A) S^{-1} f\right)(\mathbf{p})=\left(\frac{\epsilon\left(\mathbf{q}^{\eta}\right)}{\epsilon(\mathbf{p})}\right)^{1 / 2} e^{i\left\langle p^{\eta}, G a\right\rangle} D^{(j)}\left(A^{(\mu, \eta)}\left(p^{\eta}\right)^{-1} A A^{(\mu, \eta)}\left(q^{\eta}\right)\right) f\left(\mathbf{q}^{\eta}\right) .
$$

Let $T: L^{2}\left(\mathbb{R}^{3}, \mathbb{C}^{2 j+1}\right) \rightarrow L^{2}\left(\mathbb{R}^{3}, \mathbb{C}^{2 j+1}\right)$ be the unitary transformation given by

$$
(T f)(\mathbf{p}):=D^{(j)}(B(\eta \mathbf{p})) f(\mathbf{p}) .
$$

Then we have $\hat{W}^{(\mu, \eta, j)}=T S U^{(\mu, \eta, j)} S^{-1} T^{-1}$.

Let $B \in S U(2)$ and let $\mathbf{b} \in \mathbb{R}^{3}$. Since $\Lambda\left(B^{-1}\right)$ is a rotation, it is $q^{\eta}=\Lambda\left(B^{-1}\right) p^{\eta}=$ $\left(\eta \epsilon(\mathbf{p}), B^{-1} \mathbf{p}\right)$, and so we obtain

$$
\begin{aligned}
& \left(\hat{W}^{(\mu, \eta, j)}((0, \mathbf{b}), B) f\right)(\mathbf{p}) \\
& \quad=e^{-i \mathbf{p} \cdot \mathbf{b}} D^{(j)}\left(B(\eta \mathbf{p}) A(v)^{-1} B(\eta \mathbf{p})^{-1} B B\left(\eta B^{-1} \mathbf{p}\right) A(v) B\left(\eta B^{-1} \mathbf{p}\right)^{-1}\right) f\left(B^{-1} \mathbf{p}\right) .
\end{aligned}
$$

Because $B(\eta \mathbf{p})^{-1} B B\left(\eta B^{-1} \mathbf{p}\right)$ and $A(v)$ are diagonal they commute and we have

$$
\left(\hat{W}^{(\mu, \eta, j)}((0, \mathbf{b}), B) f\right)(\mathbf{p})=e^{-i \mathbf{p} \cdot \mathbf{b}} D^{(j)}(B) f\left(B^{-1} \mathbf{p}\right) .
$$

Hence, $\left.\hat{W}^{(\mu, \eta, j)}\right|_{I S U(2)}=\hat{U}_{D^{(j)}}$. 
3.4 Lemma. For every $p \in X^{(\mu, \eta)}$ we have

(i) $Q^{(\mu, \eta)}(p)=\frac{1}{\sqrt{2 \mu(\epsilon(\mathbf{p})+\mu)}}\left(\mu \sigma_{0}+\eta \sigma(p)\right)$.

(ii) $Q^{(\mu, \eta)}(p)$ is self-adjoint.

(iii) $Q^{(\mu, \eta)}$ is a cross-section, i.e.

$$
Q^{(\mu, \eta)}(p) \eta \mu e_{0}=p \quad \forall p \in X^{(\mu, \eta)},
$$

and it is the only positive cross-section.

(iv) $Q^{(\mu, \eta)}(p)^{-1}=\frac{1}{\sqrt{2 \mu(\epsilon(\mathbf{p})+\mu)}}\left((\epsilon(\mathbf{p})+\mu) \sigma_{0}-\eta \sigma(\mathbf{p})\right)$.

(v) $Q^{(\mu, \eta)}(p)^{2}=\frac{\eta}{\mu} \sigma(p)$.

Proof. Let $\mathbf{w}:=\eta \mathbf{p}$ and let $w:=\|\mathbf{w}\|=\|\mathbf{p}\|$. Then

$$
Q^{(\mu, \eta)}(p)=\left(\begin{array}{cc}
\cosh (v / 2)+\frac{w_{3}}{w} \sinh (v / 2) & \frac{w_{1}-i w_{2}}{w} \sinh (v / 2) \\
\frac{w_{1}+i w_{2}}{w} \sinh (v / 2) & \cosh (v / 2)-\frac{w_{3}}{w} \sinh (v / 2)
\end{array}\right) .
$$

Because

$$
\begin{aligned}
& \sinh (v / 2)=\left(\frac{1}{2}(\cosh (v)-1)\right)^{1 / 2}=\left(\frac{\epsilon(\mathbf{p})-\mu}{2 \mu}\right)^{1 / 2} \\
& \cosh (v / 2)=\left(\frac{1}{2}(\cosh (v)+1)\right)^{1 / 2}=\left(\frac{\epsilon(\mathbf{p})+\mu}{2 \mu}\right)^{1 / 2}
\end{aligned}
$$

the first statement follows.

It is

$$
Q^{(\mu, \eta)}\left(p^{\eta}\right) \eta \mu e_{0}=B(\eta \mathbf{p}) A(v) B(\eta \mathbf{p})^{-1} \eta \mu e_{0}=B(\eta \mathbf{p}) A(v) \eta \mu e_{0}=A^{(\mu, \eta)}\left(p^{\eta}\right) \eta \mu e_{0}=p^{\eta}
$$

thus $Q^{(\mu, \eta)}$ is indeed a cross-section. Since $A(v)$ is positive, $Q\left(p^{\eta}\right)$ is positive.

Suppose $Q^{\prime}$ is another positive cross-section. Abbreviate $Q:=Q^{(\mu, \eta)}$. Because $Q^{-1} Q^{\prime} e_{0}=e_{0}$, we have $B:=Q^{-1} Q^{\prime} \in S U(2)$. Therefore

$$
\left(Q^{\prime}\right)^{2}=Q^{\prime}\left(Q^{\prime}\right)^{*}=(Q B)(Q B)^{*}=Q B B^{*} Q^{*}=Q Q^{*}=Q^{2} .
$$

Hence, by positivity, $Q^{\prime}=Q$.

The remaining statements are easy to check. 
3.5 Definitions. A representation $W$ of $I S L(2, \mathbb{C})$ on a complex separable Hilbert space $\mathscr{H}$ is said to be a finite massive representation of $I S L(2, \mathbb{C})$ if $W$ is unitarily equivalent to a finite direct sum of irreducible strongly continuous unitary massive representations of $I S L(2, \mathbb{C})$.

Thus $W$ is a finite massive representation of $I S L(2, \mathbb{C})$ if and only if there exists a finite subset $\Omega$ of $\left\{(\mu, \eta, j): \mu>0, \eta \in\{-1,+1\}, j \in \mathbb{N}_{0} / 2\right\}$, a mapping $\nu: \Omega \rightarrow \mathbb{N}$ and a unitary $S: \mathscr{H} \rightarrow \underset{\omega \in \Omega}{\bigoplus} \nu(\omega) L^{2}\left(\mathbb{R}^{3}, \mathbb{C}^{2 \omega_{3}+1}\right)$ such that

$$
W=S^{-1} \underset{\omega \in \Omega}{\oplus} \nu(\omega) W^{(\omega)} S,
$$

where $W^{(\omega)}$ are the representations given by Lemma 3.3 . The Newton-Wigner localization for $W$ is then the projection-valued measure $E_{W}$ given by $E_{W}:=S^{-1} E S$, where $E$ is the canonical projection-valued measure. To see that $E_{W}$ is well-defined let $T$ be another unitary map such that

$$
W=T^{-1} \underset{\omega \in \Omega}{\oplus} \nu(\omega) W^{(\omega)} T .
$$

Then $T S^{-1}$ commutes with $\oplus_{\omega \in \Omega} \nu(\omega) W^{\omega}$ and by Schur's Lemma [[Fol95] 3.5] we have

$$
T S^{-1}[f]=\left[\left(\bigoplus_{\omega \in \Omega} C^{(\omega)} \otimes 1_{\omega}\right) f\right] \quad \forall[f] \in \underset{\omega \in \Omega}{\oplus} \nu(\omega) L^{2}\left(\mathbb{R}^{3}, \mathbb{C}^{2 \omega_{3}+1}\right),
$$

where $C^{(\omega)} \in \mathbb{C}^{\nu(\omega) \times \nu(\omega)}$ and $1_{\omega}$ is the identity matrix in $\mathbb{C}^{\left(2 \omega_{3}+1\right) \times\left(2 \omega_{3}+1\right)}$. Thus $T S^{-1}$ commutes with $E$. Hence

$$
E_{W}=S^{-1} E S=T^{-1} E T .
$$

The Newton-Wigner position operator for $W$ is the position operator corresponding to the Newton-Wigner localization (see Discussion 1.4).

The mass square operator $C$ for $W$ is defined as

$$
C:=S^{-1} \bigoplus_{\omega \in \Omega} \nu(\omega) \omega_{1}^{2} I_{\omega} S
$$

where $I_{\omega}$ is the identity operator in $L^{2}\left(\mathbb{R}^{3}, \mathbb{C}^{2 \omega_{3}+1}\right)$. The same argument as above shows that this operator is independent of $S$. Its spectrum is $\left\{\omega_{1}^{2}: \omega \in \Omega\right\}$ and hence finite and positive.

Basis independent descriptions for $E_{W}$ and $C$ are provided by Lemma 3.12 and Lemma 3.8 (c), respectively.

The self-adjoint operators $H, P_{a}, J_{a}, N_{a},(a=1,2,3)$, called the energy, momentum, angular momentum and boost operators of $W$, are defined via Stone's Theorem as

$$
\begin{gathered}
W((s, 0), I)=\exp (i s H), \quad W\left(\left(0, s e_{a}\right), I\right)=\exp \left(-i s P_{a}\right), \\
W\left(0, \exp \left(i s \sigma_{a} / 2\right)\right)=\exp \left(i s J_{a}\right), \quad W\left(0, \exp \left(s \sigma_{a} / 2\right)\right)=\exp \left(i s N_{a}\right),
\end{gathered}
$$

where $s \in \mathbb{R}$ (cf. [[Mut84] Eq. (2.1)]). We collect the $P_{a}, J_{a}$ and $N_{a}$ into vectors $\mathbf{P}, \mathbf{J}$ and $\mathbf{N}$, respectively. Moreover, we define $\mathbf{P}^{2}:=P_{1}^{2}+P_{2}^{2}+P_{3}^{2}$. 
The sign of energy for $W$ is defined as

$$
\operatorname{sgn} H:=\int \operatorname{sgn} d L,
$$

where $L: \mathscr{B}(\mathbb{R}) \rightarrow L(\mathscr{H})$ is the spectral measure for $H$, i.e. $H=\int$ id $d L$ and $\operatorname{sgn}(x):=x /|x|$ for $x \neq 0, \operatorname{sgn}(0):=0$. Moreover, we use this definition if $H$ is the energy operator for a time evolution.

The energy operator for $\hat{W}^{(\mu, \eta, j)}$ is given by $\mathscr{D}(\hat{H})=\left\{[f] \in L^{2}:[\xi f] \in L^{2}\right\}$, $\hat{H}[f]=[\xi f]$, where $\xi: \mathbb{R}^{3} \rightarrow \mathbb{R}, \xi(\mathbf{p}):=\eta \sqrt{\mu^{2}+\mathbf{p}^{2}}$. The spectral measure for $\hat{H}$ is $L=F \circ \xi^{-1}$, where $F: \mathscr{B}\left(\mathbb{R}^{3}\right) \rightarrow L\left(L^{2}\right), F(\Delta)[f]=\left[\chi_{\Delta} f\right]$. Hence

$$
\operatorname{sgn} \hat{H}=\sum_{\beta \in\{-1,0,1\}} \beta L\left(\operatorname{sgn}^{-1}(\{\beta\})\right)=L\left(\mathbb{R}_{>0}\right)-L\left(\mathbb{R}_{<0}\right)=\eta I,
$$

whence the sign of energy for $\underset{\omega \in \Omega}{\oplus} \nu(\omega) W^{(\omega)}$ is given by (cf. Eq. (3.4)

$$
\operatorname{sgn} H=\underset{\omega \in \Omega}{\oplus} \nu(\omega) \omega_{2} I_{\omega} .
$$

3.6 Lemma. Let $W$ be a finite massive representation of $I S L(2, \mathbb{C})$ and let $E_{W}$ be the Newton-Wigner localization for $W$. Then the following statements hold.

(a) The sign of energy for $W$ is a self-adjoint unitary bounded operator commuting with $W$ and $E_{W}$.

(b) The mass square operator for $W$ is a self-adjoint bounded operator with finite positive spectrum commuting with $W, E_{W}$ and the sign of energy.

Proof. The proof follows directly from the definitions.

3.7 Lemma. Let $W$ be a finite massive representation of $I S L(2, \mathbb{C})$ on a complex separable Hilbert space $\mathscr{H}$, let $E_{W}$ be its Newton-Wigner localization and let

$$
V(t):=W((t, 0), I), \quad U(\mathbf{b}, B):=W((0, \mathbf{b}), B), \quad t \in \mathbb{R},(\mathbf{b}, B) \in I S U(2) .
$$

Then $\left(U, E_{W}\right)$ is a localization but $\left(V, U, E_{W}\right)$ is not a causal localization.

Proof. The localization property follows from Definition 3.5. Suppose that $\left(V, U, E_{W}\right)$ is causal. Let $P_{ \pm}:=\frac{1}{2}(I \pm \operatorname{sgn}(H))$ be the projection onto positive and negative energy states. Since $V, U$ and $E_{W}$ commute with sgn $H$, they leave $\mathscr{H}_{ \pm}:=P_{ \pm}(\mathscr{H})$ invariant. From Discussion 2.4 we have $V_{ \pm}:=P_{ \pm} V P_{ \pm}=P_{ \pm}$. But then it is $V=V_{+}+V_{-}=I$ which is impossible.

Despite of this Lemma the Newton-Wigner localization is still useful for our objectives, in particular for finding relativistic extensions for $(V, U)$, see Lemma 4.7 and section 6 .

3.8 Lemma. Let $W$ be a finite massive representation of $I S L(2, \mathbb{C})$ on a complex separable Hilbert space $\mathscr{H}$. Then the following statements hold. 
(a) There are unique self-adjoint bounded operators $A$ and $B$ such that for all $[g] \in$ $\mathscr{D}(|H|)$

$$
|H| A[g]=A|H|[g]=[g], \quad B\left(|H|+C^{1 / 2}\right)[g]=\left(|H|+C^{1 / 2}\right) B[g]=[g] .
$$

Moreover, we have

$$
H \operatorname{sgn}(H) A[g]=\operatorname{sgn}(H) A H[g]=[g] \quad \forall[g] \in \mathscr{D}(H) .
$$

and

$$
\operatorname{sgn} H=H A .
$$

(b) The domain for $H^{2}$ is a dense subspace of $\mathscr{H}$ and equals the domain of $\mathbf{P}^{2}$.

(c) The mass square operator $C$ for $W$ is the unique bounded operator satisfying

$$
H^{2}[g]=\left(C+\mathbf{P}^{2}\right)[g] \quad \forall[g] \in \mathscr{D}\left(H^{2}\right) .
$$

Proof. It suffices to consider the representation $\hat{W}^{(\eta, \mu, j)}$.

(a) We have

$$
\mathscr{D}(\hat{H})=\left\{[f] \in L^{2}:\left[\eta \sqrt{\mu^{2}+|\cdot|^{2}} f\right] \in L^{2}\right\}, \quad \hat{H}[f]=\left[\eta \sqrt{\mu^{2}+|\cdot|^{2}} f\right]
$$

and

$$
\mathscr{D}\left(\hat{P}_{a}\right)=\left\{[f] \in L^{2}:\left[\left\langle\cdot, e_{a}\right\rangle f\right] \in L^{2}\right\}, \quad \hat{P}_{a}[f]=\left[\left\langle\cdot, e_{a}\right\rangle f\right]
$$

(cf. Proposition 11.5 . Also, $\mathscr{D}(|\hat{H}|)=\mathscr{D}(\hat{H}),|\hat{H}|[f]=\left[\sqrt{\mu^{2}+|\cdot|{ }^{2}} f\right]$. Plainly,

$$
\hat{A}[f]:=\left[\left(\mu^{2}+|\cdot|^{2}\right)^{-1 / 2} f\right]
$$

and

$$
\hat{B}[f]:=\left[\left(\left(\mu^{2}+|\cdot|^{2}\right)^{1 / 2}+\mu\right)^{-1} f\right]
$$

define bounded self-adjoint operators satisfying the stated equations.

Let $[g] \in \mathscr{D}(|H|)$ and let $A^{\prime}$ be another self-adjoint bounded operator satisfying the same conditions as $A$. Then $[g]=|H| A^{\prime}[g]$ and $A^{\prime}[g] \in \mathscr{D}(|H|)$ imply

$$
A[g]=A|H| A^{\prime}[g]=A^{\prime}[g] .
$$

Since $\mathscr{D}(|H|)$ is dense, it follows that $A$ is unique. Similar arguments show that $B$ is unique.

(b) Let $f \in L^{2}$ such that $\epsilon^{2} f \in L^{2}$, where $\epsilon(\mathbf{p}):=\sqrt{\mu^{2}+|\mathbf{p}|^{2}}$. Then the estimate $\epsilon \leq 1+\epsilon^{2}$ implies

$$
\|\epsilon f\|^{2} \leq\|f\|^{2}+\left\|\epsilon^{2} f\right\|<\infty .
$$

Hence $\mathscr{D}\left(\hat{H}^{2}\right)=\left\{[f] \in L^{2}:\left[\left(\mu^{2}+|\cdot|^{2}\right) f\right] \in L^{2}\right\}$. So this is a dense subspace of $L^{2}$. 
We have $\mathscr{D}\left(\hat{\mathbf{P}}^{2}\right)=\cap_{a=1}^{3} \mathscr{D}\left(\hat{P}_{a}^{2}\right)$, and $[f] \in \mathscr{D}\left(\hat{P}_{a}^{2}\right)$ if and only if $[f] \in \mathscr{D}\left(\hat{P}_{a}\right)$ and $\hat{P}_{a}[f] \in \mathscr{D}\left(\hat{P}_{a}\right)$. Let $[f] \in \mathscr{D}\left(\hat{H}^{2}\right)$. Then

$$
\left\|p_{a} f(\mathbf{p})\right\|^{2} \leq\left(\mu^{2}+|\mathbf{p}|^{2}\right)\|f(\mathbf{p})\|^{2} \leq\|f(\mathbf{p})\|^{2}+\left\|\left(\mu^{2}+|\mathbf{p}|^{2}\right) f(\mathbf{p})\right\|^{2} \quad \forall \mathbf{p} \in \mathbb{R}^{3}
$$

implies that $[f] \in \mathscr{D}\left(\hat{P}_{a}\right)$, and

$$
\left\|p_{a}^{2} f(\mathbf{p})\right\|^{2} \leq\left\|\left(\mu^{2}+|\mathbf{p}|^{2}\right) f(\mathbf{p})\right\|^{2} \quad \forall \mathbf{p} \in \mathbb{R}^{3}
$$

implies that $\hat{P}_{a}[f] \in \mathscr{D}\left(\hat{P}_{a}\right)$. Thus $[f] \in \mathscr{D}\left(\hat{\mathbf{P}}^{2}\right)$.

If $[f] \in \mathscr{D}\left(\hat{\mathbf{P}}^{2}\right)$, then $\left\||\mathbf{p}|^{2} f(\mathbf{p})\right\|^{2} / 3 \leq \sum_{a=1}^{3}\left\|p_{a}^{2} f(\mathbf{p})\right\|^{2}$ for all $\mathbf{p} \in \mathbb{R}^{3}$ shows that $\left[|\cdot|^{2} f\right] \in L^{2}$ and $\left[\left(\mu^{2}+|\cdot|^{2}\right) f\right] \in L^{2}$. Hence $[f] \in \mathscr{D}\left(\hat{H}^{2}\right)$.

(c) Since $\hat{C}[f]=\left[\mu^{2} f\right]$ for all $f \in L^{2}$, we have $\hat{H}^{2}[f]=\left[\left(\mu^{2}+|\cdot|^{2}\right) f\right]=\left(\hat{C}+\hat{P}^{2}\right)[f]$ for all $[f] \in \mathscr{D}\left(\hat{H}^{2}\right)$. Moreover, $\mathscr{D}\left(\hat{H}^{2}\right)$ is dense, so $\hat{C}$ is the only bounded operator with this property.

3.9 Note. The formulas given in part (d) and (e) of the next Theorem for finite massive representations with $\operatorname{sgn} H=+1$ (positive energies) are due to Bakamjian, Thomas and Foldy [[BT53], [Fol61] ] and we will call them the BTF formulas, cf. [[Mut84] Eq. (2.3) fol.]. Part (a) is adapted from [[Mut78 Theorem 1]. But our version holds for positive and negative energies. For the proof of (e) we follow Jor80.

3.10 Theorem. Let $W$ be a finite massive representation of $I S L(2, \mathbb{C})$ on a complex separable Hilbert space $\mathscr{H}$ and let $\mathbf{X}$ be the Newton-Wigner position operator for $W$. Then the following statements hold.

(a) There exists a dense subspace $\mathscr{D}$ of $\mathscr{H}$ such that $W \mathscr{D} \subset \mathscr{D}, \mathscr{D} \subset \mathscr{D}(A), A \mathscr{D} \subset \mathscr{D}$ and $\overline{\left.A\right|_{\mathscr{D}}}=A$ for every $A \in\{\mathbf{X}, H, \mathbf{P}, \mathbf{J}, \mathbf{N}\}$. Moreover, $A \mathscr{D} \subset \mathscr{D}$ for every $A \in\left\{\operatorname{sgn}(H),\left(|H|+C^{1 / 2}\right)^{-1}, H^{-1}, C^{-1 / 2}\right\}$.

(b) Within $\mathscr{D}$ we have the commutations relations

$$
\begin{aligned}
& {\left[P_{i}, P_{j}\right]=0 \quad\left[P_{i}, H\right]=0 \quad\left[J_{i}, H\right]=0} \\
& {\left[J_{i}, J_{j}\right]=i \varepsilon_{i j k} J_{k} \quad\left[J_{i}, P_{j}\right]=i \varepsilon_{i j k} P_{k} \quad\left[J_{i}, N_{j}\right]=i \varepsilon_{i j k} N_{k}} \\
& {\left[H, N_{j}\right]=-i P_{j} \quad\left[N_{i}, N_{j}\right]=-i \varepsilon_{i j k} J_{k} \quad\left[P_{i}, N_{j}\right]=-i \delta_{i j} H} \\
& {\left[X_{i}, X_{j}\right]=0 \quad\left[X_{i}, P_{j}\right]=i \delta_{i j} \quad\left[X_{i}, H\right]=i P_{i} H^{-1}} \\
& {\left[J_{i}, X_{j}\right]=i \varepsilon_{i j k} X_{k}}
\end{aligned}
$$

where a sum over a repeated index is understood and $\varepsilon_{i j k}$ is the Levi-Civita symbol.

(c) There is a unique bounded self-adjoint operator $\mathbf{S}$, called the spin vector for $W$, satisfying

$$
\mathbf{S}[f]=(\mathbf{J}-\mathbf{X} \times \mathbf{P})[f] \quad \forall[f] \in \mathscr{D},
$$

where $(\mathbf{A} \times \mathbf{B})_{k}:=\varepsilon_{k a b} A_{a} B_{b}$. 
(d) For every $[f] \in \mathscr{D}$ it is

$$
\mathbf{N}[f]=\left(\frac{1}{2}(H \mathbf{X}+\mathbf{X} H)+\frac{\operatorname{sgn}(H)}{|H|+C^{1 / 2}} \mathbf{P} \times \mathbf{S}\right)[f] .
$$

(e) For every $[f] \in \mathscr{D}$ it is

$$
\mathbf{X}[f]=\left(\frac{1}{2}\left(H^{-1} \mathbf{N}+\mathbf{N} H^{-1}\right)-\frac{1}{H C^{1 / 2}\left(|H|+C^{1 / 2}\right)} \mathbf{P} \times(H \mathbf{J}+\mathbf{P} \times \mathbf{N})\right)[f] .
$$

(f) $\mathbf{S}^{2}$ commutes with $\left(W, E_{W}\right)$.

(g) The representation $W^{(\eta, \mu, j)}$ occurs in the decomposition of $W$ if and only if $\eta$ is an eigenvalue of $\operatorname{sgn} H, \mu^{2}$ is an eigenvalue of $C$ and $j(j+1)$ is an eigenvalue of $\mathbf{S}^{2}$.

Proof. For (a) - (f) it will be sufficient to prove the claims for $\hat{W}^{(\eta, \mu, j)}$, where $\eta \in$ $\{-1,1\}, \mu>0$ and $j \in \mathbb{N}_{0} / 2$ are arbitrary but fixed.

We will show that $\mathscr{D}:=C_{c}^{\infty}\left(\mathbb{R}^{3}\right)($ cf. [[BR86] Ch.11 $\S 1(6)$ p. 319]) satisfies (a). In this particular representation it is clear that $\hat{W}^{(\eta, \mu, j)} \mathscr{D} \subset \mathscr{D}$ and $\mathscr{D} \subset \mathscr{D}(\hat{A})$ for the generators $\hat{A}$ of $\hat{W}^{(\eta, \mu, j)}$. Then [[RS80] Theorem VIII.11 p. 269] implies that $\mathscr{D}$ is a common core for $\hat{H}, \hat{\mathbf{P}}, \hat{\mathbf{J}}$ and $\hat{\mathbf{N}}$.

By the same arguments as in the proof of Lemma 1.16 we see that $\mathscr{D}$ is a core for $\hat{\mathbf{X}}$ and that $\hat{X}_{k}[f]=\left[i \partial_{k} f\right]$ for all $[f] \in \mathscr{D}$. In particular $\hat{X}_{a} \mathscr{D} \subset \mathscr{D}$. Also, (cf. Proposition 11.5

$$
\hat{H}[f]=[\eta \epsilon(\cdot) f], \quad \hat{P}_{k}[f]=\left[\left\langle\cdot, e_{k}\right\rangle f\right] \quad \forall[f] \in \mathscr{D}
$$

where $\epsilon(\mathbf{p}):=\sqrt{\mu^{2}+|\mathbf{p}|^{2}}$. Clearly, $\hat{H} \mathscr{D} \subset \mathscr{D}$ and $\hat{P}_{a} \mathscr{D} \subset \mathscr{D}$. For $[f] \in \mathscr{D}$ it is

$$
\begin{aligned}
\left(\hat{J}_{k} f\right)(\mathbf{p}) & =-i \partial_{\alpha}\left(D^{(j)}\left(e^{i \alpha \sigma_{k} / 2}\right) f\left(e^{-i \alpha \sigma_{k} / 2} \mathbf{p}\right)\right)_{\alpha=0} \\
& =\left(\left[\hat{S}_{k}-(\hat{\mathbf{P}} \times \hat{\mathbf{X}})_{k}\right] f\right)(\mathbf{p})
\end{aligned}
$$

where $\hat{S}_{k}$ are the matrix multiplication operators corresponding to the matrices $L_{k}$ which are defined by

$$
D^{(j)}\left(e^{i \alpha \sigma_{k} / 2}\right)=e^{i \alpha L_{k}} .
$$

This also shows that $\hat{J}_{a} \mathscr{D} \subset \mathscr{D}$. Since $\left[\hat{X}_{i}, \hat{P}_{j}\right]=i \delta_{i j}$ in $\mathscr{D}$, we have $\hat{\mathbf{P}} \times \hat{\mathbf{X}}=-\hat{\mathbf{X}} \times \hat{\mathbf{P}}$. Thus

$$
\hat{S}_{k}[f]=\left(\hat{J}_{k}-(\hat{\mathbf{X}} \times \hat{\mathbf{P}})_{k}\right)[f] \quad \forall[f] \in \mathscr{D} .
$$

Because $\mathscr{D}$ is dense $\mathbf{S}$ is the unique bounded operator satisfying this equation. This proves (c). The properties of $\mathbf{L}$ imply (f) (see Appendix C). (g) is not difficult to see. 
Let $A_{\alpha}:=\exp \left(\alpha \sigma_{k} / 2\right), p^{\eta}:=(\eta \epsilon(\mathbf{p}), \mathbf{p}), q^{\eta}:=A_{\alpha}^{-1} p^{\eta}$ and $\mathbf{q}^{\eta}:=\left(q^{\eta}\right)^{\downarrow}$. We need to compute

$$
\left(\hat{N}_{k} f\right)(\mathbf{p})=-i \partial_{\alpha}\left(\left(\frac{\epsilon\left(\mathbf{q}^{\eta}\right)}{\epsilon(\mathbf{p})}\right)^{1 / 2} D^{(j)}\left(Q\left(p^{\eta}\right)^{-1} A_{\alpha} Q\left(q^{\eta}\right)\right) f\left(\mathbf{q}^{\eta}\right)\right)_{\alpha=0}
$$

Since $\left.\partial_{\alpha} q^{\eta}\right|_{\alpha=0}=-\left(p_{k}, \eta \epsilon(\mathbf{p}) e_{k}\right)$ and $\epsilon\left(\mathbf{q}^{\eta}\right)=\eta q_{0}^{\eta}$, we find

$$
\left(\hat{N}_{k} f\right)(\mathbf{p})=-i\left(\frac{-\eta p_{k}}{2 \epsilon(\mathbf{p})}+d D^{(j)}(I) \circ \partial_{\alpha}\left[Q\left(p^{\eta}\right)^{-1} A_{\alpha} Q\left(q^{\eta}\right)\right]_{\alpha=0}-\eta \epsilon(\mathbf{p}) \partial_{k}\right) f(\mathbf{p}) .
$$

The first and the third term can be written as $\frac{1}{2}\left(H X_{k}+X_{k} H\right) f$. To evaluate the second term we use

$$
Q\left(p^{\eta}\right)=\frac{1}{\sqrt{2 \mu(\epsilon(\mathbf{p})+\mu)}}\left(\mu \sigma_{0}+\eta \sigma\left(p^{\eta}\right)\right) .
$$

This gives

$$
Q\left(q^{\eta}\right)=\frac{1}{\sqrt{2 \mu\left(\epsilon\left(\mathbf{q}^{\eta}\right)+\mu\right)}}\left(\mu \sigma_{0}+\eta A_{k}^{-1} \sigma\left(p^{\eta}\right) A_{k}^{-1}\right)
$$

and we obtain

$$
\left.\partial_{\alpha} A_{\alpha} Q\left(q^{\eta}\right)\right|_{\alpha=0}=\frac{\eta p_{k}}{2(\epsilon(\mathbf{p})+\mu)} Q\left(p^{\eta}\right)+\frac{1}{2 \sqrt{2 \mu(\epsilon(\mathbf{p})+\mu)}}\left(\mu-\eta \sigma\left(p^{\eta}\right)\right) \sigma_{k} .
$$

Because

$$
Q\left(p^{\eta}\right)^{-1}=\frac{1}{\sqrt{2 \mu(\epsilon(\mathbf{p})+\mu)}}\left((\epsilon(\mathbf{p})+\mu) \sigma_{0}-\eta \sigma(\mathbf{p})\right)
$$

it is not difficult to find that

$\left.\partial_{\alpha}\left(Q\left(p^{\eta}\right)^{-1} A_{\alpha} Q\left(q^{\eta}\right)\right)\right|_{\alpha=0}=\frac{\eta p_{k}}{2(\epsilon(\mathbf{p})+\mu)}-\frac{\eta}{2(\epsilon(\mathbf{p})+\mu)} \sigma(\mathbf{p}) \sigma_{k}=\frac{\eta}{2(\epsilon(\mathbf{p})+\mu)} i(\mathbf{p} \times \sigma)_{k}$.

Using $\frac{1}{2} d D^{(j)}(I) \circ \sigma_{k}=L_{k}$ proves $(\mathrm{d})$. And now it is clear that $\hat{N}_{a} \mathscr{D} \subset \mathscr{D}$.

The operators $\hat{H}^{-1}$ and $\left(|\hat{H}|+C^{1 / 2}\right)^{-1}$ are the self-adjoint bounded operators given by

$$
\hat{H}^{-1}[f]:=\left[\eta\left(\mu^{2}+|\cdot|^{2}\right)^{-1 / 2} f\right], \quad\left(|\hat{H}|+C^{1 / 2}\right)^{-1}[f]:=\left[\left(\left(\mu^{2}+|\cdot|^{2}\right)^{-1 / 2}+\mu\right)^{-1} f\right] .
$$

Since the functions $\mathbf{p} \mapsto \eta\left(\mu^{2}+|\mathbf{p}|^{2}\right)^{-1 / 2}$ and $\mathbf{p} \mapsto\left(\left(\mu^{2}+|\mathbf{p}|^{2}\right)^{-1 / 2}+\mu\right)^{-1}$ have derivatives up to all orders, it is clear that the corresponding operators leave $\mathscr{D}$ invariant. Plainly, $\operatorname{sgn}(\hat{H}) \mathscr{D} \subset \mathscr{D}$ and $C^{-1 / 2} \mathscr{D} \subset \mathscr{D}$. So the proof of (a) is complete.

The commutations relation in (b) are well-known, see, e.g., [Fol61]. 
To prove (e) we follow [Jor80]. The subsequent formulas are understood to hold on the subspace $\mathscr{D}$. By (d) we have

$$
\mathbf{P} \times \mathbf{N}=H(\mathbf{P} \times \mathbf{X})+\frac{\operatorname{sgn} H}{|H|+C^{1 / 2}}\left(\mathbf{P}(\mathbf{P} \cdot \mathbf{S})-\mathbf{P}^{2} \mathbf{S}\right),
$$

where we used $(\mathbf{A} \times(\mathbf{B} \times \mathbf{C}))_{k}=A_{a} B_{k} C_{a}-(\mathbf{A} \cdot \mathbf{B}) C_{k}$. Because $\mathbf{P} \cdot \mathbf{S}=\mathbf{P} \cdot \mathbf{J}$ and $\mathbf{P} \times \mathbf{X}=\mathbf{S}-\mathbf{J}$ we have

$$
\mathbf{S}=\frac{\operatorname{sgn} H}{C^{1 / 2}}\left(H \mathbf{J}+\mathbf{P} \times \mathbf{N}-\frac{\operatorname{sgn} H}{|H|+C^{1 / 2}} \mathbf{P}(\mathbf{P} \cdot \mathbf{J})\right) .
$$

Since $[\mathbf{X}, H]=i \frac{\mathbf{P}}{H}$, it is

$$
\frac{1}{2}(H \mathbf{X}+\mathbf{X} H)=H \mathbf{X}+i \frac{\mathbf{P}}{2 H} .
$$

Now the formula for $\mathbf{N}$ can be solved for $\mathbf{X}$. We find

$$
\mathbf{X}=\frac{1}{H} \mathbf{N}-i \frac{\mathbf{P}}{2 H^{2}}-\frac{1}{H C^{1 / 2}\left(|H|+C^{1 / 2}\right)} \mathbf{P} \times(H \mathbf{J}+\mathbf{P} \times \mathbf{N}) .
$$

Using

$$
\left[\mathbf{N}, \frac{1}{H}\right]=\frac{1}{H}[H, \mathbf{N}] \frac{1}{H}=-i \frac{\mathbf{P}}{H^{2}}
$$

proves (e).

3.11 Note. The spin vector $\mathbf{S}$ for a finite massive representation $W$ is related to the Pauli-Lubanski four-vector $w:=\left(w_{0}, \mathbf{w}\right)$, where

$$
w_{0}:=\mathbf{P} \cdot \mathbf{S}, \quad \mathbf{w}:=H \mathbf{J}+\mathbf{P} \times \mathbf{N} .
$$

Using Eq. (3.7) and $\mathbf{P} \times \mathbf{X}=\mathbf{S}-\mathbf{J}$ we find (cf. [[Jor80] Eq. (2.6)])

$$
\mathbf{w}=\frac{\operatorname{sgn}(H)}{|H|+C^{1 / 2}} \mathbf{P}(\mathbf{P} \cdot \mathbf{S})+\operatorname{sgn}(H) C^{1 / 2} \mathbf{S} .
$$

Because $\left[H, S_{k}\right]=\left[P_{j}, S_{k}\right]=0$ it is then easy to check that $w^{2}:=w_{0}^{2}-\mathbf{w}^{2}=-C \mathbf{S}^{2}$.

3.12 Lemma. Let $W$ be a finite massive representation of $I S L(2, \mathbb{C})$ on a complex separable Hilbert space $\mathscr{H}$. Then the Newton-Wigner localization is the unique projectionvalued measure whose corresponding position operator is given by the BTF formula (3.6.).

Proof. See Discussion 1.4 and Lemma 1.5 .

3.13 Discussion. Another formula for the Newton-Wigner position operator is given by

$$
\begin{aligned}
\mathbf{X} & =\mathbf{Q}-\mathbf{P} \times(\mathbf{J}-\mathbf{Q} \times \mathbf{P}) C^{-1 / 2}\left(|H|+C^{1 / 2}\right)^{-1} \\
& =\mathbf{Q}-C^{-1 / 2}\left(|H|+C^{1 / 2}\right)^{-1} \mathbf{P} \times(\mathbf{J}-\mathbf{Q} \times \mathbf{P})
\end{aligned}
$$


where $\mathbf{Q}:=\frac{1}{2}\left(H^{-1} \mathbf{N}+\mathbf{N} H^{-1}\right)$, cf. [[Mut84] Eq. (2.4)]. These can be easily seen by considering

$$
\mathbf{P} \times \mathbf{N}=-\mathbf{N} \times \mathbf{P}, \quad \frac{1}{H} \mathbf{N}=\mathbf{Q}+\frac{i \mathbf{P}}{2 H^{2}}, \quad[H, \mathbf{Q}]=-\frac{i \mathbf{P}}{H} .
$$




\section{Relativistic Causal Localizations}

4.1 Definition. Let $W$ be a finite massive representation of $I S L(2, \mathbb{C})$ on a complex separable Hilbert space $\mathscr{H}$ and let $E: \mathscr{B}\left(\mathbb{R}^{3}\right) \rightarrow L(\mathscr{H})$ be a projection-valued measure. Then $(W, E)$ is called a relativistic causal localization if $\left(\left.W\right|_{\mathscr{T}},\left.W\right|_{I S U(2)}, E\right)$ is a finite causal localization, where $\left.W\right|_{\mathscr{T}}$ is the time evolution part of $W$, i.e. $t \mapsto$ $W((t, 0), I)$.

Notice, that by means of the representation property and the fact that a matrix $B \in S U(2)$ acts as a rotation in space, $V:=\left.W\right|_{\mathscr{T}}$ always commutes with $U:=\left.W\right|_{I S U(2)}$. Indeed,

$$
\begin{aligned}
V(t) U(\mathbf{b}, B) & =W((t, 0), I) W((0, \mathbf{b}), B)=W((t, \mathbf{b}), B) \\
& =W((0, \mathbf{b}), B) W((t, 0), I)=U(\mathbf{b}, B) V(t) \quad \forall t \in \mathbb{R},(\mathbf{b}, B) \in I S U(2) .
\end{aligned}
$$

A tuple $(V, U, E)$ is said to be a relativistic extendable causal localization if there exists a relativistic causal localization $(W, E)$ such that $\left(\left.W\right|_{\mathscr{T}},\left.W\right|_{I S U(2)}\right)=(V, U)$. Note: Since we only consider finite localizations and massive representations, we do not find the need to repeat the terms finite and massive in these definitions.

4.2 Lemma. Let $(W, E)$ be a relativistic causal localization such that $\left(\left.W\right|_{I S U(2)}, E\right)$ is given in the coordinate space representation form and let $V$ be the time evolution part of $W$. Then there exist self-adjoint matrices $M$ and $N$ such that in the helicity representation

$$
V^{h}(t)[f]=\left[e^{i t(M+|\cdot| N)} f\right] \quad \forall[f] \in \mathscr{H},
$$

$M N+N M=0, N^{2}=I$ and $C^{h}[f]=\left[M^{2} f\right]$ for all $[f] \in \mathscr{H}$. Here, $C^{h}$ is the mass square operator for $W$ given in the helicity representation. In particular, $C$ commutes with $(W, E)$.

Proof. Considering Theorem 2.17 we only need to show that $M N+N M=0, N^{2}=I$ and $C^{h}[f]=\left[M^{2} f\right]$ for all $[f] \in \mathscr{H}$.

We have $\mathscr{D}:=\mathscr{D}\left(\left(\mathbf{P}^{h}\right)^{2}\right)=\left\{[f] \in L^{2}:\left[|\cdot|^{2} f\right] \in L^{2}\right\}$, and by Lemma 3.8

$$
C^{h}[f]=\left(\left(H^{h}\right)^{2}-\sum_{i=1}^{3}\left(P_{i}^{h}\right)^{2}\right)[f] \quad \forall[f] \in \mathscr{D} .
$$

Since $C$ commutes with $U$, there exists an $F \in L^{\infty}\left(\mathbb{R}^{3}, \oplus_{\omega \in \Omega} \nu(\omega) L\left(\mathbb{C}^{2 \omega_{3}+1}\right)\right)$ such that in the helicity representation $C^{h}[f]=[F(\cdot) f]$ (see Theorem G.3). Put $G: \mathbb{R}^{3} \rightarrow$ $\oplus_{\omega \in \Omega} \nu(\omega) L\left(\mathbb{C}^{2 \omega_{3}+1}\right)$,

$$
G(\mathbf{p}):=(M+|\mathbf{p}| N)^{2}-|\mathbf{p}|^{2} I .
$$

Then $[(F-G) f]=0$ for all $[f] \in \mathscr{D}$, since

$$
H^{h}[f]=[(M+|\cdot| N) f] \quad \forall[f] \in \mathscr{D}\left(H^{h}\right) .
$$


We show that $F=G$ a.e. Let $\|\cdot\|_{F}$ be the Frobenius norm, i.e. $\|A\|_{F}^{2}:=\operatorname{tr}\left(A^{*} A\right)$. For $n \in \mathbb{N}$ put

$$
S_{n}:=\left\{\mathbf{p} \in \mathbb{R}^{3}:\|F(\mathbf{p})-G(\mathbf{p})\|_{F}^{2}>\frac{1}{n}\right\} .
$$

Suppose $\lambda\left(S_{n}\right)>0$. Then there exists a compact $K \subset S_{n}$ such that $0<\lambda(K)<\infty$. Let $f_{i}:=\chi_{K} b_{i}$, where $\left\{b_{i}\right\}_{i}$ is an orthonormal basis in $\underset{\omega \in \Omega}{\oplus} \nu(\omega) \mathbb{C}^{2 \omega_{3}+1}$. Since $f_{i} \in \mathscr{D}$, we have

$$
0=\sum_{i}\left\|\left[(F-G) f_{i}\right]\right\|^{2}=\int_{K}\|F(\mathbf{p})-G(\mathbf{p})\|_{F}^{2} d \lambda(\mathbf{p}) \geq \lambda(K) \frac{1}{n}>0,
$$

which is impossible. So $S_{n}$ is a null set for each $n \in \mathbb{N}$. Because the union of all these $S_{n}$ is still a null set, we obtain $F=G$ a.e. This implies $G \in L^{\infty}\left(\mathbb{R}^{3}, \underset{\omega \in \Omega}{\oplus} \nu(\omega) L\left(\mathbb{C}^{2 \omega_{3}+1}\right)\right)$, in other words $\lambda\left(T_{\beta}\right)=0$ for some $\beta>0$, where

$$
T_{\beta}:=\left\{\mathbf{p} \in \mathbb{R}^{3}:\|G(\mathbf{p})\|>\beta\right\} .
$$

Let $C_{0}:=M^{2}, C_{1}:=M N+N M, C_{2}:=N^{2}-I$, then

$$
G(\mathbf{p})=C_{0}+C_{1}|\mathbf{p}|+C_{2}|\mathbf{p}|^{2} \quad \forall \mathbf{p} \in \mathbb{R}^{3} .
$$

Suppose $C_{2} \neq 0$. Then there exists a vector $x$ with $|x|=1$ such that $C_{2} x \neq 0$. But then the estimate

$$
\|G(\mathbf{p})\| \geq\|G(\mathbf{p}) x\|=\left\|C_{0} x+C_{1} x|\mathbf{p}|+C_{2} x|\mathbf{p}|^{2}\right\| \quad \forall \mathbf{p} \in \mathbb{R}^{3}
$$

shows that for each $\beta>0$ there exists an $r>0$ such that $\left\{\mathbf{p} \in \mathbb{R}^{3}:|\mathbf{p}|>r\right\} \subset T_{\beta}$. This contradicts $\lambda\left(T_{\beta}\right)=0$ for some $\beta>0$. Hence $C_{2}=0$. Similarly, we have $C_{1}=0$. Hence, $M N+N M=0, N^{2}=I$ and

$$
C^{h}[f]=\left[M^{2} f\right] \quad \forall[f] \in \mathscr{D} .
$$

Since $\mathscr{D}$ is dense, this holds for every $[f] \in \mathscr{H}$.

4.3 Corollary. Let $(W, E)$ be an irreducible relativistic causal localization. Then the mass square operator for $W$ is given by $C=\mu^{2} I$ for some $\mu>0$.

Proof. By Lemma $4.2 C$ commutes with $(W, E)$. Then by the irreducibility of $(W, E)$ and the Spectral Theorem $C=\mu^{2} I$.

4.4 Corollary. Let $(V, U, E)$ be an irreducible finite causal localization. If there exists a relativistic extension $(W, E)$ for $(V, U, E)$ then the mass square operator for $W$ is given by $C=\mu^{2} I$ for some $\mu>0$.

Proof. If $(V, U, E)$ is irreducible, then $(W, E)$ is irreducible. Thus Corollary 4.3 completes the proof. 
4.5 Lemma. Separation of bosons and fermions. Let $(U, E)$ be a finite localization and let $V$ be a time evolution. If $(V, U, E)$ is irreducible, then $U$ contains only half integer or only integer spins

Moreover, if $(W, E)$ is an irreducible relativistic localization, then $W$ contains only half integer or only integer spins.

Proof. We may assume that $(U, E)$ is given in the coordinate space representation. Let $P_{b}$ and $P_{f}$ be the orthogonal projection onto the boson space and fermion space respectively, i.e. in the momentum space representation

$$
\hat{P}_{b}[f]:=\left[M_{b} f\right], \quad \hat{P}_{f}[f]:=\left[M_{f} f\right],
$$

where $M_{b}$ and $M_{f}$ are the matrices defined as

$$
\begin{aligned}
\left\langle k, \kappa, r\left|M_{b}\right| j, \iota, s\right\rangle & := \begin{cases}\delta_{k j} \delta_{\kappa \iota} \delta_{r s}, & j \in \mathbb{N}_{0}, \\
0, & j \notin \mathbb{N}_{0},\end{cases} \\
\left\langle k, \kappa, r\left|M_{f}\right| j, \iota, s\right\rangle: & = \begin{cases}\delta_{k j} \delta_{\kappa \iota} \delta_{r s}, & j \notin \mathbb{N}_{0}, \\
0, & j \in \mathbb{N}_{0} .\end{cases}
\end{aligned}
$$

Clearly, $P_{b}$ and $P_{f}$ commute with $(U, E)$. Since $V$ commutes with $U$, it is of the form

$$
V^{h}(t)[f]=\left[F_{t}(|\cdot|) f\right]
$$

where for each $t \in \mathbb{R}, \rho \mapsto F_{t}(\rho)$ is a measurable bounded matrix-valued function such that (see Lemma 1.13)

$$
\left\langle k, \kappa, r\left|F_{t}\right| j, \iota, s\right\rangle=0 \quad \forall r \neq s .
$$

This implies that $P_{b}$ and $P_{f}$ commute with $V$. By the irreducibility of $(V, U, E)$ and Schur's Lemma (Appendix I) we have either $P_{b}=0$ or $P_{f}=0$.

Now consider an irreducible relativistic localization $(W, E)$. We may assume that $W=\oplus_{\omega \in \Omega} \nu(\omega) W^{(\omega)}$. Since $(U, E):=\left(\left.W\right|_{I S U(2)}, E\right)=\left(\oplus_{\omega \in \Omega} \nu(\omega) U_{D^{\left(\omega_{3}\right)}}, E\right)$ is a localization, Mackey's Imprimitivity Theorem implies that there exists a unitary $T$ commuting with $U$ such that $T E T^{-1}$ is the canonical projection-valued measure. By the same reason as above $P_{b}$ and $P_{f}$ commute with $T$. Thus $P_{b}$ and $P_{f}$ commute with $E$, and it is easy to see that they commute with $W$. Again by Schur's Lemma we have $P_{b}=0$ or $P_{f}=0$.

4.6 Lemma. Let $(U, E)$ be a finite localization and let $V$ be a time evolution. If there exists a bounded operator $C \geq 0$ such that $H^{2}=C+\mathbf{P}^{2}$ then $C$ commutes with $U$ and $V$. Moreover, the sign of energy, $\operatorname{sgn} H$, is a self-adjoint unitary bounded operator commuting with $C, V$ and $U$. 
Proof. For $f \in C_{c}^{\infty}\left(\mathbb{R} \times \mathbb{R}^{3} \times S U(2)\right), \phi \in \mathscr{H}$ put

$$
\phi_{f}:=\int_{\Sigma} f(t, \mathbf{b}, B) V(t) U(\mathbf{b}, B) \phi d t d \mathbf{b} d B, \quad \Sigma:=\mathbb{R} \times \mathbb{R}^{3} \times S U(2),
$$

where $d B$ denotes the Haar measure on $S U(2)$, and $d \mathbf{x}$ denotes the Lebesgue measure on $\mathbb{R}^{3}$. Let

$$
\mathscr{D}:=\operatorname{span}\left\{\phi_{f}: f \in C_{c}^{\infty}\left(\mathbb{R} \times \mathbb{R}^{3} \times S U(2)\right), \phi \in \mathscr{H}\right\} .
$$

With some minor modifications to the proof of Stone's Theorem [[RS80] VIII.8] we see that $\mathscr{D}$ is a dense subset of $\mathscr{H}$ such that $V \mathscr{D} \subset \mathscr{D}, U \mathscr{D} \subset \mathscr{D}, H \mathscr{D} \subset \mathscr{D}$ and $P_{i} \mathscr{D} \subset \mathscr{D}$. Thus for each $\psi \in \mathscr{D}$ the expression $[H, U] \psi$ makes sense and by Stone's Theorem it is

$$
[H, U] \psi=\lim _{s \rightarrow 0}(-i)\left[\frac{V(s)-I}{s}, U\right] \psi=0 \quad \forall \psi \in \mathscr{D} .
$$

Hence $\left[H^{2}, U\right] \psi=0$ for all $\psi \in \mathscr{D}$. By the same reasoning $\left[H^{2}, V\right] \psi=0$ for all $\psi \in \mathscr{D}$. Since $U \mathscr{D}\left(\mathbf{P}^{2}\right) \subset \mathscr{D}\left(\mathbf{P}^{2}\right)$ and $\left[\mathbf{P}^{2}, U\right] f=0$ for all $f \in \mathscr{D}\left(\mathbf{P}^{2}\right)$, which can be verified easily in the momentum space representation, we have $\left[\mathbf{P}^{2}, U\right] \psi=0$ for all $\psi \in \mathscr{D}$. As $V$ commutes with $U$, each $V(t)$ is in the momentum space representation a matrix multiplication operator commuting with $\mathbf{P}^{2}$ on $\mathscr{D}\left(\mathbf{P}^{2}\right)$. Hence $\left[\mathbf{P}^{2}, V\right] \psi=0$ for all $\psi \in$ $\mathscr{D}$. Considering that $C \psi=\left(H^{2}-\mathbf{P}^{2}\right) \psi$ for all $\psi \in \mathscr{D}$, we have $[C, V] \psi=[C, U] \psi=0$ for all $\psi \in \mathscr{D}$ and by continuity $[C, V]=[C, U]=0$.

Let $L$ be the projection-valued measure on $(\mathbb{R}, \mathscr{B}(\mathbb{R}))$ such that $H=\int$ id $d L$. Then $\operatorname{sgn} H=\int \operatorname{sgn}(\cdot) d L$. In order to show that $[\operatorname{sgn} H, A]=0$ for $A \in\{C, V, U\}$ it suffices to proof that $[L, A]=0$, see, e.g. [Cas11] Ch. $2(11)(\mathrm{g})]$. By the Spectral Theorem [ Cas11] Ch. $5(7)]$ we have $[L, A]=0$ if and only if $A H \subset H A$. The last condition is true, since for $\psi \in \mathscr{D}(H)$ we have

$$
\lim _{t \rightarrow 0} \frac{V(t)-I}{i t} A \psi=A \lim _{t \rightarrow 0} \frac{V(t)-I}{i t} \psi,
$$

which implies that $A \psi \in \mathscr{D}(H)$ and $H A \psi=A H \psi$.

Because $\mathbf{P}^{2}>0$ and $C \geq 0$ it is $H^{2}=C+\mathbf{P}^{2}>0$. Then, from $H^{2}=\int \mathrm{id}^{2} d L$ we have $L\left(\left\{\operatorname{id}^{2} \leq 0\right\}\right)=0$, whence $L(\{0\})=0$, which then implies that $\operatorname{sgn} H$ is unitary (cf. Cas11] Ch. $4(2)(\mathrm{f})$ and a minor modification of (i)).

4.7 Lemma. Let $(U, E)$ be a finite localization and let $V$ be a time evolution. Then $(V, U)$ has a relativistic extension $W$ if and only if

(i) There exists a bounded operator $C$ having finite positive spectrum such that $H^{2}=$ $C+\mathbf{P}^{2}$.

(ii) There exists a projection-valued measure $F: \mathscr{B}\left(\mathbb{R}^{3}\right) \rightarrow L(\mathscr{H})$ such that $(U, F)$ is a localization and $[\operatorname{sgn} H, F]=[C, F]=0$.

Moreover, if (i) and (ii) are satisfied then the following statements are true. 
(a) There exists one and only one relativistic extension $W_{F}$ such that $F$ is the NewtonWigner localization for $W_{F}$.

(b) If there is a unitary $S$ commuting with $U$ and $V$, then $S W_{F} S^{-1}$ is also a relativistic extension and $S W_{F} S^{-1}=W_{S F S^{-1}}$.

(c) If $W$ is a relativistic extension of $(V, U)$, then there exists a unitary $S$ commuting with $U$ and $V$ such that $W=W_{S F S^{-1}}=S W_{F} S^{-1}$.

Proof. The "if" part of the Lemma: Lemma 4.6 implies that $\operatorname{sgn} H$ is a self-adjoint unitary bounded operator commuting with $V$ and $U$, and $C$ commutes with $V, U$ and sgn $H$. By the Spectral Theorem there are orthogonal projections $C_{\gamma}$ commuting with $(V, U, F)$ such that $C=\sum_{\gamma \in \sigma(C)} \gamma C_{\gamma}, C_{\gamma} C_{\gamma^{\prime}}=\delta_{\gamma \gamma^{\prime}} C_{\gamma}$ and $\sum_{\gamma \in \sigma(C)} C_{\gamma}=I$. For $\eta \in\{-1,1\}$ let $P_{\eta}:=\frac{1}{2}(I+\eta \operatorname{sgn} H)$ be the orthogonal projections onto positive and negative energy states. The $P_{\eta}$ commute with $(V, U, F), P_{\eta} P_{-\eta}=0$ and $P_{\eta}+P_{-\eta}=I$. Also, by the Spectral Theorem, $P_{\eta}$ and $C_{\gamma}$ commute.

We may assume that $(U, F)$ is in the following form:

$$
U=\bigoplus_{j \in \Omega} I_{j} \otimes U_{D^{(j)}}, \quad F=\bigoplus_{j \in \Omega} I_{j} \otimes E^{(j)},
$$

where $I_{j} \in \mathbb{C}^{\nu_{j} \times \nu_{j}}$ is the identity matrix. The projections are then given by

$$
C_{\gamma}=\bigoplus_{j \in \Omega} M_{\gamma}^{(j)} \otimes I^{(j)}, \quad P_{\eta}=\bigoplus_{j \in \Omega} N_{\eta}^{(j)} \otimes I^{(j)},
$$

where, for each $j, M_{\gamma}^{(j)}, N_{\eta}^{(j)} \in \mathbb{C}^{\nu_{j} \times \nu_{j}}$ are orthogonal projections commuting with each other and $I^{(j)}$ is the identity operator acting on $L^{2}\left(\mathbb{R}^{3}, \mathbb{C}^{2 j+1}\right)$. Put

$$
W_{F}:=\sum_{\eta, \gamma}\left(\oplus_{j \in \Omega} I_{j} \otimes W^{(\sqrt{\gamma}, \eta, j)}\right) P_{\eta} C_{\gamma}
$$

Plainly, $P_{\eta}$ and $C_{\gamma}$ commute with $\underset{j \in \Omega}{\oplus} I_{j} \otimes W^{(\sqrt{\gamma}, \eta, j)}$, so $W_{F}$ is a finite massive representation of $I S L(2, \mathbb{C})$ and $\left.W_{F}\right|_{I S U(2)}=U$. The energy operator $H_{F}$ for $W_{F}$ is then given by

$$
H_{F}=\sum_{\eta, \gamma}\left(\oplus_{j \in \Omega} I_{j} \otimes \eta \sqrt{\gamma I^{(j)}+\left(P^{(j)}\right)^{2}}\right) P_{\eta} C_{\gamma}
$$

By means of

$$
\begin{aligned}
\left(\sum_{\gamma}\left(\oplus_{j \in \Omega} I_{j} \otimes \sqrt{\gamma I^{(j)}+\left(P^{(j)}\right)^{2}}\right) C_{\gamma}\right)^{2} & =\sum_{\gamma} \oplus_{j \in \Omega} M_{\gamma}^{(j)} \otimes\left(\gamma I^{(j)}+\left(P^{(j)}\right)^{2}\right) \\
& =\sum_{\gamma} \gamma C_{\gamma}+\sum_{\gamma} C_{\gamma}\left(\oplus_{j \in \Omega} I_{j} \otimes\left(P^{(j)}\right)^{2}\right) \\
& =C+\mathbf{P}^{2}
\end{aligned}
$$


and the uniqueness of the positive square root we have

$$
H_{F}=\sum_{\eta} \eta \sqrt{C+\mathbf{P}^{2}} P_{\eta}=\sum_{\eta} \eta|H| P_{\eta}=\sum_{\eta} H P_{\eta}=H .
$$

Hence $W_{F}$ is a relativistic extension for $(V, U)$.

If we simultaneously diagonalize $M_{\gamma}^{(j)}$ and $N_{\eta}^{(j)}$, then we see that $W_{F}$ is unitarily equivalent to a finite direct sum of $W^{(\mu, \eta, j)}$, and since this transform leaves $U$ and $F$ invariant, we see that $F$ is the Newton-Wigner localization for $W_{F}$.

The "only if" part of the Lemma: Let $C$ be the mass square operator for $W$ and let $F$ be the Newton-Wigner localization for $W$. Then (i) and (ii) follow from Lemma 3.8 and Definition 3.5 .

(a) The existence has already been shown above. To show uniqueness let $W^{\prime}$ be another extension such that $F$ is the Newton-Wigner localization for $W^{\prime}$. From the BTF formula the boosts for $W^{\prime}$ and $W_{F}$ must be identical, hence $W^{\prime}=W_{F}$.

(b) Let $S$ be a unitary operator commuting with $(V, U)$ and let $\mathbf{N}$ be the boost of $W_{F}$. Then from the BTF formula $S \mathbf{N} S^{-1}$ must be the boost for $W_{S F S^{-1}}$, hence $S W_{F} S^{-1}=W_{S F S^{-1}}$.

(c) Let $W$ be a relativistic extension for $(V, U)$ and let $E_{W}$ be its Newton-Wigner localization. Then $C$ is the mass square operator for $W$. Thus $C$ and $\operatorname{sgn} H$ commute with $\left(W, E_{W}\right)$ and $\left(W_{F}, F\right)$, which is to say that both pairs decompose in the same manner and we may assume in the following that $W$ and $W_{F}$ contain a single mass $\mu$ and a single sign of energy $\eta$.

Assume that $\left(W_{F}, F\right)$ is in standard form, i.e.

$$
W_{F}=\underset{j \in \Omega_{3}}{\oplus} \nu(j) W^{(\mu, \eta, j)}, \quad F=\underset{j \in \Omega_{3}}{\oplus} \nu(j) E^{(j)},
$$

where $\Omega_{3}=\left\{j_{1}, j_{2}, \ldots, j_{n}\right\}$ is a finite subset of $\mathbb{N}_{0} / 2$ such that $j_{1}<j_{2}<\ldots<$ $j_{n}$ and $E^{(j)}$ is the canonical projection-valued measure, i.e. multiplication with the characteristic function.

By the definition of a finite massive representation there exists a unitary operator $S$ such that

$$
S\left(W, E_{W}\right) S^{*}=\left(\underset{j^{\prime} \in \Omega_{3}^{\prime}}{\oplus} \nu^{\prime}\left(j^{\prime}\right) W^{\left(\mu, \eta, j^{\prime}\right)}, \underset{j^{\prime} \in \Omega_{3}^{\prime}}{\oplus} \nu\left(j^{\prime}\right) E^{\left(j^{\prime}\right)}\right),
$$

where $\Omega_{3}^{\prime}=\left\{j_{1}^{\prime}, j_{2}^{\prime}, \ldots, j_{m}^{\prime}\right\}$ is a finite subset of $\mathbb{N}_{0} / 2$ such that $j_{1}^{\prime}<j_{2}^{\prime}<\ldots<j_{m}^{\prime}$. Plainly,

$$
F=\underset{j^{\prime} \in \Omega_{3}^{\prime}}{\bigoplus} \nu\left(j^{\prime}\right) E^{\left(j^{\prime}\right)}
$$

Let

$$
U^{\prime}:=\left.\underset{j^{\prime} \in \Omega_{3}^{\prime}}{\bigoplus} \nu^{\prime}\left(j^{\prime}\right) W^{\left(\mu, \eta, j^{\prime}\right)}\right|_{I S U(2)} .
$$


By Mackey's Imprimitivity Theorem $\left(U, E_{W}\right)$ and $\left(U^{\prime}, F\right)$ are induced representations of unitarily equivalent $S U(2)$ representations. Hence, $\Omega_{3}^{\prime}=\Omega_{3}$ and the multiplicities $\nu^{\prime}$ and $\nu$ coincide. In other words it is

$$
W_{F}=\underset{j^{\prime} \in \Omega_{3}^{\prime}}{\bigoplus} \nu^{\prime}\left(j^{\prime}\right) W^{\left(\mu, \eta, j^{\prime}\right)},
$$

whence

$$
S\left(W, E_{W}\right) S^{*}=\left(W_{F}, F\right) .
$$

Clearly, $S$ commutes with $U$ and $V$, since $W$ and $W_{F}$ are extensions of $U$ and $V$. Now (b) completes the proof.

4.8 Lemma. Let $A_{i}, B_{i} \in L\left(\mathscr{H}_{i}\right)$ be orthogonal projections such that $A_{i} \leq B_{i}, \quad(i=$ 1,2). Then $A_{1} \otimes A_{2} \leq B_{1} \otimes B_{2}$.

Proof. By Lemma 2.9 we have

$$
\left(B_{1} \otimes B_{2}\right)\left(A_{1} \otimes A_{2}\right)=\left(B_{1} A_{1}\right) \otimes\left(B_{2} A_{2}\right)=A_{1} \otimes A_{2} .
$$

Since $A_{1} \otimes A_{2}$ and $B_{1} \otimes B_{2}$ are orthogonal projections, Lemma 2.9 completes the proof.

4.9. Theorem. Causal localizations via tensor products. Let $(U, E)$ be a localization and let $V$ be a time evolution on a separable complex Hilbert space $\mathscr{H}$. Let $D: S U(2) \rightarrow \mathbb{C}^{d \times d}$ be a finite dimensional unitary representation of $S U(2)$. Put

$$
\left(V^{\prime}, U^{\prime}, E^{\prime}\right):=\left(V \otimes I, U \otimes D^{\prime}, E \otimes I\right),
$$

where $D^{\prime}(\mathbf{b}, B):=D(B)$ for $(\mathbf{b}, B) \in I S U(2)$ and I denotes the identity operator acting on $\mathbb{C}^{d}$. More precisely,

$$
V^{\prime}(t):=V(t) \otimes I, \quad U^{\prime}(\mathbf{b}, B):=U(\mathbf{b}, B) \otimes D(B), \quad E^{\prime}(\Delta):=E(\Delta) \otimes I,
$$

for all $t \in \mathbb{R},(\mathbf{b}, B) \in I S U(2)$ and $\Delta \in \mathscr{B}\left(\mathbb{R}^{3}\right)$. Then the following holds.

(a) $\left(U^{\prime}, E^{\prime}\right)$ is a localization on $\mathscr{H} \otimes \mathbb{C}^{d}$.

(b) If $(V, U, E)$ is a causal finite localization, then $\left(V^{\prime}, U^{\prime}, E^{\prime}\right)$ is also a causal finite localization.

(c) If $(V, U, E)$ is a relativistic extendable causal localization, then $\left(V^{\prime}, U^{\prime}, E^{\prime}\right)$ is also a relativistic extendable causal localization.

Proof. (a) It is easy to see that $U^{\prime}$ is a strongly continuous unitary representation of $I S U(2)$ and that $E^{\prime}$ is a projection-valued measure. Let $\Delta \in \mathscr{B}\left(\mathbb{R}^{3}\right)$ and $(\mathbf{b}, B) \in$ $I S U(2)$. Since $(U, E)$ is a localization, we have

$$
\begin{aligned}
U^{\prime}(\mathbf{b}, B) E^{\prime}(\Delta) U^{\prime}(\mathbf{b}, B)^{-1} & =\left(U(\mathbf{b}, B) \otimes D^{\prime}(B)\right)(E(\Delta) \otimes I)\left(U(\mathbf{b}, B) \otimes D^{\prime}(B)\right)^{-1} \\
& =\left(U(\mathbf{b}, B) E(\Delta) U(\mathbf{b}, B)^{-1}\right) \otimes I \\
& =E((\mathbf{b}, B) \cdot \Delta) \otimes I=E^{\prime}((\mathbf{b}, B) \cdot \Delta) .
\end{aligned}
$$


Hence $\left(U^{\prime}, E^{\prime}\right)$ is a localization.

(b) Because $U$ is a finite localization, there exists a finite dimensional representation $\tilde{D}$ of $S U(2)$ such that $U \cong U_{\tilde{D}}$. Then $U^{\prime} \cong U_{\tilde{D} \otimes D}$ and since $\tilde{D} \otimes D$ is a finite dimensional representation, $\left(U^{\prime}, E^{\prime}\right)$ is a finite localization.

Lemma 4.8 implies that

$$
\begin{aligned}
V^{\prime}(t) E^{\prime}(\Delta) V^{\prime}(-t) & =(V(t) \otimes I)(E(\Delta) \otimes I)(V(-t) \otimes I) \\
& =(V(t) E(\Delta) V(-t)) \otimes I \leq E\left(\Delta_{t}\right) \otimes I=E^{\prime}\left(\Delta_{t}\right) .
\end{aligned}
$$

Thus $\left(V^{\prime}, U^{\prime}, E^{\prime}\right)$ is a causal finite localization.

(c) Let $W$ be a finite massive representation of $\operatorname{ISL}(2, \mathbb{C})$ extending $(V, U)$ and let $F$ be its Newton-Wigner localization. The energy operator $H^{\prime}$ for $V^{\prime}$ is given by $H^{\prime}=H \otimes I$, and the momentum operator $\mathbf{P}^{\prime}$ for $U^{\prime}$ is given by $\mathbf{P}^{\prime}=\mathbf{P} \otimes I$. Let $C$ be the mass square operator for $W$. We have $H^{2}=C+\mathbf{P}^{2}$ and by Lemma 4.7 $[\operatorname{sgn} H, F]=[C, F]=0$. Then $C^{\prime}:=C \otimes I$ is a bounded operator with finite positive spectrum satisfying $\left(H^{\prime}\right)^{2}=C^{\prime}+\mathbf{P}^{\prime 2}$. Put $F^{\prime}:=F \otimes I$. By the same arguments as above $\left(U^{\prime}, F^{\prime}\right)$ is localization. Applying Lemma 4.7 for $\left(V^{\prime}, U^{\prime}\right)$ completes the proof, since it is clear that $\left[\operatorname{sgn} H^{\prime}, F^{\prime}\right]=\left[C^{\prime}, F^{\prime}\right]=0$.

4.10 Remark. This result implies that if there exists a causal finite localization which is indeed the case as we will see - then there are infinitely many inequivalent causal localizations.

4.11 Remark. Theorem 4.9 leads directly to our main result. In the next section we show that the Dirac system is a relativistic extendable causal localization. By applying this Theorem to the Dirac system we obtain the Dirac tensor systems. Moreover, it is shown that these and the Dirac system are up to unitary equivalence the only irreducible relativistic extendable causal localizations. 


\section{Relativistic Extendable Causal Localizations and the Dirac System}

In this section we determine all relativistic extendable causal localizations (Theorem 5.10. These are up to unitary equivalence direct sums of Dirac- and Dirac tensor systems, which are defined in 5.4 and 5.7. We like to stress that we consider only finite localizations and massive representations of $I S L(2, \mathbb{C})$.

5.1 Theorem. Let $h: \mathbb{C} \rightarrow \mathbb{C}^{d \times d}$ be a matrix-valued function such that

(a) $h(x)$ is self-adjoint for all $x \in \mathbb{R}$.

(b) $h(z)=A z+B$ for all $z \in \mathbb{C}$, for some matrices $A, B \in \mathbb{C}^{d \times d}$.

(c) There exists a positive matrix $C$ such $h(z)^{2}=z^{2}+C$ for all $z \in \mathbb{C}$.

Then $d=2 m$ for some integer $m \in \mathbb{N}$ and there exists a unitary matrix $U$ independent of z such that

$$
U^{*} h(z) U=\bigoplus_{i=1}^{m}\left(\begin{array}{cc}
\sqrt{c_{i}} & z \\
z & -\sqrt{c_{i}}
\end{array}\right) \quad \forall z \in \mathbb{C},
$$

where $c_{1}, \ldots, c_{m}$ are the eigenvalues with multiplicities of $C$.

Proof. (a) implies that $A$ and $B$ are self-adjoint. By (b) and (c) we must have $A^{2}=I_{d}$, $A B+B A=0$ and $B^{2}=C$. Thus $A$ is unitarily equivalent to $\left(\begin{array}{cc}I_{l} & 0 \\ 0 & -I_{m}\end{array}\right)$ for some $l, m \in \mathbb{N}_{0}$ such that $l+m=d$, where $I_{a}$ denotes the identity matrix on $\mathbb{C}^{a \times a}$. In this basis we may write

$$
B=\left(\begin{array}{ll}
B_{1} & B_{2} \\
B_{3} & B_{4}
\end{array}\right)
$$

where $B_{1} \in \mathbb{C}^{l \times l}, B_{2} \in \mathbb{C}^{l \times m}, B_{3} \in \mathbb{C}^{m \times l}, B_{4} \in \mathbb{C}^{m \times m}$. Since $B$ is self-adjoint, we have $B_{3}=B_{2}^{*}$. The condition $A B+B A=0$ implies $B_{1}=0$ and $B_{4}=0$. So, from $B^{2}=C$ we obtain

$$
C=\left(\begin{array}{cc}
C_{1} & 0 \\
0 & C_{2}
\end{array}\right)
$$

where $C_{1}:=B_{2} B_{2}^{*}$ and $C_{2}=B_{2}^{*} B_{2}$. Since $C$ is positive, $C_{1}$ and $C_{2}$ are positive and they are unitarily equivalent to some diagonal matrices. The unitary transform that diagonalizes both matrices respects the block form of $A$ and $B$. Therefore, without loss of generality, we may assume that

$$
A=\left(\begin{array}{cc}
I_{l} & 0 \\
0 & -I_{m}
\end{array}\right), \quad B=\left(\begin{array}{cc}
0 & B_{2} \\
B_{2}^{*} & 0
\end{array}\right), \quad C=\left(\begin{array}{cc}
\operatorname{diag}\left(c_{1}, \ldots, c_{l}\right) & 0 \\
0 & \operatorname{diag}\left(c_{l+1}, \ldots, c_{d}\right)
\end{array}\right),
$$

where $c_{1}, \ldots, c_{d}>0$. 
Assume that $l>m$. Then there exists a nonzero vector $v \in \mathbb{C}^{l}$ such that $B_{2}^{*} v=0$. But then $C_{1} v=B_{2} B_{2}^{*} v=0$ which is impossible. Similarly the case $l<m$ can be excluded. Hence, we must have $l=m$, in particular $d=2 m$.

Let $P \in \mathbb{R}^{m \times m}$ be the positive diagonal matrix such that $P^{2}=C_{1}^{-1}$. Then $Y:=P B_{2}$ is unitary: $Y Y^{*}=P B_{2} B_{2}^{*} P^{*}=C_{1} P^{2}=I$ which also implies that $Y^{*} Y=I$. We have $C_{1} Y=C_{1} P B_{2}=P C_{1} B_{2}=P B_{2} B_{2}^{*} B_{2}=Y C_{2}$, hence $C_{2}$ is unitarily equivalent to $C_{1}$. Thus, using the unitary transform $\left(\begin{array}{cc}I_{m} & 0 \\ 0 & Y\end{array}\right)$, we may assume that $C_{1}=C_{2}=$ $\operatorname{diag}\left(c_{1}, \ldots, c_{m}\right)$.

Moreover, this implies that $B_{2}$ is normal. By the Spectral Theorem $B_{2}=V K V^{*}$ for some unitary $V$ and diagonal $K$. Since $B_{2} B_{2}^{*}=\operatorname{diag}\left(c_{1}, \ldots, c_{m}\right)$, we may assume that

$$
K=\operatorname{diag}\left(\sqrt{c_{1}} e^{i \varphi_{1}}, \ldots, \sqrt{c_{m}} e^{i \varphi_{m}}\right),
$$

for some $\varphi_{k} \in \mathbb{R}$. Put $W:=\operatorname{diag}\left(e^{i \varphi_{1} / 2}, \ldots, e^{i \varphi_{m} / 2}\right)$, then

$$
h(z)=\left(\begin{array}{cc}
V W & 0 \\
0 & V W^{*}
\end{array}\right)\left(\begin{array}{cc}
I_{m} z & D \\
D & -I_{m} z
\end{array}\right)\left(\begin{array}{cc}
W^{*} V^{*} & 0 \\
0 & W V^{*}
\end{array}\right),
$$

where $D:=\operatorname{diag}\left(\sqrt{c_{1}}, \ldots, \sqrt{c_{m}}\right)$.

Finally, we observe that

$$
\frac{1}{\sqrt{2}}\left(\begin{array}{cc}
I_{m} & I_{m} \\
I_{m} & -I_{m}
\end{array}\right)\left(\begin{array}{cc}
I_{m} z & D \\
D & -I_{m} z
\end{array}\right) \frac{1}{\sqrt{2}}\left(\begin{array}{cc}
I_{m} & I_{m} \\
I_{m} & -I_{m}
\end{array}\right)=\left(\begin{array}{cc}
D & I_{m} z \\
I_{m} z & -D
\end{array}\right),
$$

and

$$
\left(\begin{array}{cc}
D & I_{m} z \\
I_{m} z & -D
\end{array}\right) \cong \bigoplus_{i=1}^{m}\left(\begin{array}{cc}
\sqrt{c_{i}} & z \\
z & -\sqrt{c_{i}}
\end{array}\right)
$$

5.2 Lemma. Let $M, N \in \mathbb{C}^{m \times m}$ be self-adjoint matrices such that $M N+N M=0$, $N^{2}=I$ and $M^{2}>0$. Let $h, Y: \mathbb{R}_{\geq 0} \rightarrow \mathbb{C}^{m \times m}$,

$$
h(\rho):=M+\rho N, \quad Y(\rho):=\frac{1}{\sqrt{2|h(\rho)|(|h(\rho)|+|M|)}}\left(\frac{|h(\rho)|+|M|}{|M|} M+\rho N\right) .
$$

Then for all $\rho \geq 0$ we have

(a) $Y(\rho)$ is self-adjoint and unitary.

(b) $Y(\rho) h(\rho) Y(\rho)^{-1}=\frac{|h(\rho)|}{|M|} M$.

(c) $\left[Y(\rho), M^{2}\right]=0$.

Proof. Since $|h(\rho)|=\sqrt{M^{2}+\rho^{2}}$, the Square Root Lemma implies that $|h(\rho)|$ commutes with $|M|$, so $Y$ is well-defined. Obviously, $Y(\rho)$ is self-adjoint. Since $\left[M^{2}, N\right]=0$, we have $[|M|, N]=[|h(\rho)|, N]=0$ again by the Square Root Lemma. Then it is easy to prove the unitarity, (b) and (c). 
5.3 Theorem. Let $(U, E)$ be the coordinate space representation of a finite localization and let $V$ be a time evolution. Then there exists a relativistic causal localization $(W, E)$ extending $(V, U, E)$ if and only if there are self-adjoint matrices $M$ and $N$ satisfying the following conditions:

(a) In the helicity representation it is

$$
V^{h}(t)[f]=\left[e^{i t(M+|\cdot| N)} f\right] \quad \forall[f] \in \mathscr{H} .
$$

(b) $M N+N M=0, N^{2}=I$ and $M^{2}>0$.

(c) There are constants $c_{\kappa \iota}^{(k)} \in \mathbb{C}$ such that

$$
\langle k, \kappa, r|M| j, \iota, s\rangle=c_{\kappa \iota}^{(k)} \delta_{r s} \delta_{k j} .
$$

(d) There are constants $A_{\kappa \iota}^{(k, j)} \in \mathbb{C}$ such that

$$
\langle k, \kappa, r|N| j, \iota, s\rangle=\delta_{r s} D_{s, 1}^{(k, j)} A_{\kappa \iota}^{(k, j)},
$$

where

$$
D_{s, l}^{(k, j)}:=(-1)^{j-s} \sqrt{2 l+1}\left(\begin{array}{rrr}
j & k & l \\
-s & s & 0
\end{array}\right) .
$$

Proof. In the following we will occasionally omit the multiplicity indices $\kappa$ and $\iota$ - we may think of them as matrix-indices.

The "only if" part: $M N+N M=0$ and $N^{2}=I$ have been proven in Lemma 4.2. Since the mass square operator is positive and $C^{h}[f]=\left[M^{2} f\right]$ for all $[f] \in \mathscr{H}$, we have $M^{2}>0$. This proves (a) and (b). (c) is part of Theorem 2.17 .

(d) By Lemma $4.2 C$ commutes with $(W, E)$, so we may assume that $C=\mu^{2} I$ for some $\mu>0$. Theorem 2.14 gives

$$
\left(\Psi_{r s}^{(k, j)}\right)_{\kappa \iota}(z):=\left\langle k, \kappa, r\left|e^{i t h(z)}\right| j, \iota, s\right\rangle=\delta_{r s} \sum_{l=|j-k|}^{j+k} D_{s, l}^{(k, j)} z^{l} f_{t, \kappa, l}^{(k, j, l)}\left(z^{2}\right),
$$

where $h(z):=M+z N$ for $z \in \mathbb{C}$. The derivative with respect to $z$ gives

$$
\Psi_{r s}^{(k, j) \prime}(z)=\delta_{r s} \sum_{l=|j-k|}^{j+k} D_{s, l}^{(k, j)}\left(l z^{l-1} f_{t}^{(k, j, l)}\left(z^{2}\right)+2 z^{l+1} f_{t}^{\prime(k, j, l)}\left(z^{2}\right)\right),
$$

and for $z=0$ we have

$$
\Psi_{r s}^{(k, j) \prime}(0)=\delta_{r s} D_{s, 1}^{(k, j)} f_{t}^{(k, j, 1)}(0) .
$$

On the other hand, since $h(z)^{2}=\left(\mu^{2}+z^{2}\right) I$, we have

$$
\begin{aligned}
\Psi(z) & =e^{i t h(z)}=\sum_{n=0}^{\infty}(-1)^{n} \frac{t^{2 n}}{(2 n) !}\left(\mu^{2}+z^{2}\right)^{n} I+i h(z) \sum_{n=0}^{\infty}(-1)^{n} \frac{t^{2 n+1}}{(2 n+1) !}\left(\mu^{2}+z^{2}\right)^{n}, \\
\Psi^{\prime}(0) & =i N \sum_{n=0}^{\infty}(-1)^{n} \frac{t^{2 n+1}}{(2 n+1) !}\left(\mu^{2}\right)^{n}=i t N S\left(t^{2} \mu^{2}\right),
\end{aligned}
$$


where

$$
S(x):=\sum_{n=0}^{\infty}(-1)^{n} \frac{x^{n}}{(2 n+1) !} .
$$

Choose $t>0$ such that $S\left(t^{2} \mu^{2}\right) \neq 0$. Then we find

$$
N=\Psi^{\prime}(0) \frac{1}{i t S\left(t^{2} \mu^{2}\right)}
$$

and therefore

$$
\langle k, \kappa, r|N| j, \iota, s\rangle=\delta_{r s} D_{s, 1}^{(k, j)} f_{t, \kappa, \iota}^{(k, j)}(0) \frac{1}{i t S\left(t^{2} \mu^{2}\right)} .
$$

This proves $(d)$.

The "if" part: By (a) we have $H^{h}[f]=[(M+|\cdot| N) f]$ for all $[f] \in \mathscr{D}\left(H^{h}\right)$. (b) implies $H^{2}=C+\mathbf{P}^{2}$, where $C^{h}[f]=\left[M^{2} f\right]$ for all $[f] \in L^{2}$. Since $M^{2}>0$ and $M$ is self-adjoint, $C$ has a finite positive spectrum.

Put $T^{h}[f]:=[Y(\cdot) f]$, with $Y$ from Lemma 5.2 , and $F:=T E T^{-1}$. Using (c), (d) and Lemma 1.13, we find that $T$ commutes with $U$, hence $(U, F)$ is a finite localization. Since $C$ commutes with $T$ and $E$, it commutes with $F$. We have $\operatorname{sgn}\left(H^{h}\right)[f]=\left[\frac{h(|\cdot|)}{|h(|\cdot|)|} f\right]$, thus $\left(T^{h}\right)^{-1} \operatorname{sgn}\left(H^{h}\right) T^{h}[f]=[M /|M| f]$. This implies that $[\operatorname{sgn}(H), F]=0$. By Lemma $4.7(V, U)$ has a relativistic extension.

We show that $(V, U, E)$ is a causal localization. Let $\Psi_{t}(z):=e^{i t(M+z N)}$. Clearly, (a) implies part (a) of Theorem 2.14. By (b) we have $\|N\|^{2}=\left\|N^{2}\right\|=1$, hence

$$
\left\|e^{i t(M+z N)}\right\| \leq e^{|t| \mid M \|} e^{|t||z|} \quad \forall z \in \mathbb{C} .
$$

This shows part (b) of Theorem 2.14.

It remains to show that there are entire functions $f_{t}^{(k, j, l)}$ such that

$$
\left\langle k, \kappa, r\left|\Psi_{t}(z)\right| j, \iota, s\right\rangle=\delta_{r s} \sum_{l=|j-k|}^{j+k} D_{s, l}^{(k, j)} z^{l} f_{t, \kappa, \iota}^{(k, j, l)}\left(z^{2}\right) \quad \forall z \in \mathbb{C} .
$$

We have

$$
\Psi_{t}(z)=C\left(t^{2}\left(M^{2}+z^{2}\right)\right)+i t(M+z N) S\left(t^{2}\left(M^{2}+z^{2}\right)\right)
$$

where

$$
C(x):=\sum_{n=0}^{\infty}(-1)^{n} \frac{x^{n}}{(2 n) !}, \quad S(x):=\sum_{n=0}^{\infty}(-1)^{n} \frac{x^{n}}{(2 n+1) !} .
$$

Because of (c) we have

$$
\begin{aligned}
\left\langle k, \kappa, r\left|C\left(t^{2}\left(M^{2}+z^{2}\right)\right)\right| j, \iota, s\right\rangle & =a_{\kappa \iota}^{(k)}\left(z^{2}\right) \delta_{r s} \delta_{k j}, \\
\left\langle k, \kappa, r\left|S\left(t^{2}\left(M^{2}+z^{2}\right)\right)\right| j, \iota, s\right\rangle & =b_{\kappa \iota}^{(k)}\left(z^{2}\right) \delta_{r s} \delta_{k j},
\end{aligned}
$$

where $a_{\kappa \iota}^{(k)}$ and $b_{\kappa \iota}^{(k)}$ are entire functions (the time dependency is omitted). Then (d) implies

$$
\left\langle k, \kappa, r\left|\Psi_{t}(z)\right| j, \iota, s\right\rangle=g_{\kappa \iota}^{(k)}\left(z^{2}\right) \delta_{r s} \delta_{k j}+i t z \delta_{r s} D_{s, 1}^{(k, j)} \tilde{A}_{\kappa \iota}^{(k, j)}\left(z^{2}\right)
$$


where

$$
g_{\kappa \iota}^{(k)}:=a_{\kappa \iota}^{(k)}+i t \sum_{\lambda} c_{\kappa \lambda}^{(k)} b_{\lambda \iota}^{(k)}, \quad \tilde{A}_{\kappa \iota}^{(k, j)}:=\sum_{\lambda} A_{\kappa \lambda}^{(k, j)} b_{\lambda \iota}^{(k)} .
$$

Using

$$
D_{s, 0}^{(k, k)}=\frac{(-1)^{2 k}}{\sqrt{2 k+1}}
$$

(cf. E.1), it is easy to see that

$$
f^{(k, j, l)}:=(-1)^{2 k} \sqrt{2 k+1} g^{(k)} \delta_{k j} \delta_{l 0}+i t \tilde{A}^{(k, j)} \delta_{l 1},
$$

solves Eq. (5.1). This completes the proof, but we show how the $f_{t}^{(k, j, l)}$ have been found.

Inverting Eq. (5.1) (see Remark 2.15) and using (5.2) gives

$$
z^{l} f^{(k, j, l)}\left(z^{2}\right)=g^{(k)}\left(z^{2}\right) \delta_{k j} \sum_{s} D_{s l}^{(k, k)}+i t z \tilde{A}^{(k, j)}\left(z^{2}\right) \sum_{s} D_{s l}^{(k, j)} D_{s 1}^{(k, j)} .
$$

Since $\sum_{s} D_{s, l}^{(k, k)}=0$ for $l \geq 1$ (see Lemma E.4) and

$$
\sum_{s} D_{s l}^{(k, j)} D_{s 1}^{(k, j)}=\delta_{l 1} \quad \text { for }|j-k| \leq l \leq j+k
$$

we have

$$
z^{l} f^{(k, j, l)}\left(z^{2}\right)=g^{(k)}\left(z^{2}\right) \delta_{k j}(-1)^{2 k} \sqrt{2 k+1} \delta_{l 0}+i t z \tilde{A}^{(k, j)}\left(z^{2}\right) \delta_{l 1}
$$

for $|j-k| \leq l \leq j+k$.

5.4. The Dirac system. Let $U:=U_{D^{(1 / 2)}} \oplus U_{D^{(1 / 2)}}$ and let $E$ be the canonical projection-valued measure. Consider the time evolution $V$, which in the momentum space representation is given by $\hat{V}(t):=e^{i t \hat{H}}$, where $\hat{H}$ is the Dirac Operator [Tha92] Eq. (1.41)] defined as

$$
\hat{H}[f]:=[h(\cdot) f]
$$

and $h: \mathbb{R}^{3} \rightarrow \mathbb{C}^{4 \times 4}$,

$$
h(\mathbf{p}):=\left(\begin{array}{cc}
\mu I_{2} & \sigma(\mathbf{p}) \\
\sigma(\mathbf{p}) & -\mu I_{2}
\end{array}\right)
$$

where $\sigma(\mathbf{p}):=\sum_{i=1}^{3} p_{i} \sigma_{i}$ and $\mu>0$. More precisely,

$$
\left\langle\frac{1}{2}, \kappa, r|h(\mathbf{p})| \frac{1}{2}, \iota, s\right\rangle=\left\{\begin{array}{cl}
\mu \delta_{r s}, & \kappa=\iota=1 \\
-\mu \delta_{r s}, & \kappa=\iota=2 \\
\sigma(\mathbf{p})_{r s} & \kappa \neq \iota .
\end{array}\right.
$$

Every system that is unitarily equivalent to $(V, U, E)$ is then called a Dirac system with mass $\mu$. The system $(V, U, E)$ itself is called the standard Dirac system with mass $\mu$. 
In the helicity representation the Dirac operator has the form $H^{h}[f]=[\Phi(|\cdot|) f]$, where $\Phi: \mathbb{R}_{\geq 0} \rightarrow \mathbb{C}^{4 \times 4}$,

$$
\langle 1 / 2, \kappa, r|\Phi(\rho)| 1 / 2, \iota, s\rangle:=\delta_{r s} \mu\left(\sigma_{3}\right)_{\kappa \iota}+\delta_{r s} 2 s \rho\left(\sigma_{1}\right)_{\kappa \iota} .
$$

In Theorem 5.6 it is proven that the Dirac system is an irreducible relativistic extendable causal localization. Moreover, we show that the Dirac system is the only irreducible finite causal localization with spin $1 / 2$ that has a relativistic extension.

5.5. Notation. Consider

$$
H_{0}:=\nu_{\ell} \mathbb{C}^{2 \ell+1} \oplus \nu_{\ell+1} \mathbb{C}^{2(\ell+1)+1} \oplus \ldots \oplus \nu_{J} \mathbb{C}^{2 J+1},
$$

where $\nu_{k} \in \mathbb{N}_{0}, J \in \mathbb{N}_{0} / 2$ and $\ell:=0$ if $J \in \mathbb{N}$, and $\ell:=1 / 2$ otherwise (cf. 1.6). Let $A \in L\left(H_{0}\right)$ be a matrix satisfying

$$
\langle k, \kappa, r|A| j, \iota, s\rangle=0 \quad \text { for } r \neq s,
$$

such as $M$ or $N$ in Theorem 5.3 . It will be convenient to use the following matrix notation. For $s \in[k] \cap[j]$, where $[k]:=\{-k,-k+1, \ldots,+k\}$, define the matrices $A_{s}^{(k, j)} \in \mathbb{C}^{\nu_{k} \times \nu_{j}}$ as

$$
\left(A_{s}^{(k, j)}\right)_{\kappa \iota}:=\langle k, \kappa, s|A| j, \iota, s\rangle .
$$

Put

$$
A_{s}:=\left(\begin{array}{cccc}
A_{s}^{(J, J)} & A_{s}^{(J, J-1)} & \cdots & A_{s}^{(J,|s|)} \\
A_{s}^{(J-1, J)} & A_{s}^{(J-1, J-1)} & \cdots & A_{s}^{(J-1,|s|)} \\
\vdots & \vdots & \ddots & \vdots \\
A_{s}^{(|s|, J)} & A_{s}^{(|s|, J-1)} & \cdots & A_{s}^{(|s|,|s|)}
\end{array}\right) .
$$

Note that $A_{s}^{(k, j)}$ for $k<|s|$ or $j<|s|$ makes no sense. Also, if $\nu_{k}=0$ or $\nu_{j}=0$ then $A_{s}^{(k, j)}$ is not defined and should not appear in $A_{s}$.

If $B \in L\left(H_{0}\right)$ is another matrix satisfying Eq. (5.6) then it is easy to see that $A_{s} B_{s}=(A B)_{s}$. Moreover, if $A$ is self-adjoint and $B$ is unitary, then $A_{s}$ and $B_{s}$ are selfadjoint and unitary, respectively. In this notation Lemma 1.14 states that an operator commutes with $(U, E)$ if and only if it is a matrix multiplication operator corresponding to a matrix $M$ satisfying Eq. (5.6) and

$$
M_{s}=\operatorname{diag}\left(M^{(J)}, \ldots, M^{(|s|)}\right),
$$

where $\left(M^{(k)}\right)_{\kappa \iota}=\langle k, \kappa, s|M| k, \iota, s\rangle$ for some $s \in[k]$. If $\nu_{k}=0$ then $M^{(k)}$ is not defined and should not appear in $M_{s}$.

5.6 Theorem. The following statements hold.

(a) The Dirac system is an irreducible relativistic extendable causal localization.

(b) If $(V, U, E)$ is an irreducible relativistic extendable causal localization such that $1 / 2$ is the highest spin occurring in $U$, then $(V, U, E)$ is unitarily equivalent to the Dirac System. 
Proof. Let us start with (b). We may assume that $(U, E)$ is in the coordinate space representation form. By Corollary $4.4(V, U, E)$ contains a single mass $\mu>0$ and by Lemma 4.5 we may assume that

$$
U=\nu_{1 / 2} U_{D^{(1 / 2)}}
$$

for some $\nu_{1 / 2} \in \mathbb{N}$.

We use Notation 5.5. By Theorem 5.3 there are self-adjoint matrices $M$ and $N$ such that $V^{h}(t)[f]=\left[e^{i t(M+|\cdot| N)} f\right], N_{s}^{2}=I, N_{s} M_{s}+M_{s} N_{s}=0, M_{s}^{2}=\mu^{2} I$, and

$$
M_{s}=M^{(1 / 2)}, \quad N_{s}^{(1 / 2,1 / 2)}=D_{s, 1}^{(1 / 2,1 / 2)} A^{(1 / 2,1 / 2)},
$$

for $s \in\{-1 / 2,1 / 2\}$. Using the symmetries of the Wigner $3 j$ symbol (see Appendix E) we find

$$
N_{-s}^{(1 / 2,1 / 2)}=-N_{s}^{(1 / 2,1 / 2)} .
$$

Theorem 5.1 for $z \mapsto N_{s}^{(1 / 2,1 / 2)} z+M_{s}$ implies $\nu_{1 / 2}=2 \nu$ for some $\nu \in \mathbb{N}$ and there exists a unitary matrix $R^{(1 / 2)}$ such that

$$
R^{(1 / 2)} N_{1 / 2}^{(1 / 2,1 / 2)} R^{(1 / 2) *}=S_{1}:=\left(\begin{array}{cc}
0 & I_{\nu} \\
I_{\nu} & 0
\end{array}\right), \quad R^{(1 / 2)} M^{(1 / 2)} R^{(1 / 2) *}=\mu S_{3}:=\mu\left(\begin{array}{cc}
I_{\nu} & 0 \\
0 & -I_{\nu}
\end{array}\right) .
$$

Since the matrix multiplication operator corresponding to $R_{s}:=R^{(1 / 2)}$ commutes with $(U, E)$, we may assume that $M^{(1 / 2)}$ and $N_{1 / 2}^{(1 / 2,1 / 2)}$ are already in this form.

If $\nu>1$, then $(V, U, E)$ is reducible, since $T_{s}:=T$, where $T_{\kappa \iota}:=\delta_{\kappa \iota}\left(\delta_{\kappa, 1}+\delta_{\kappa, \nu+1}\right)$, defines an orthogonal projection onto an invariant closed subspace for $(V, U, E)$ (cf. Appendix (I). So we must have $\nu=1$ and

$$
N_{1 / 2}=\sigma_{1}, \quad N_{-1 / 2}=-\sigma_{1} \quad M_{1 / 2}=\mu \sigma_{3}, \quad M_{-1 / 2}=\mu \sigma_{3} .
$$

In other words it is

$$
\langle 1 / 2, \kappa, r|N| 1 / 2, \iota, s\rangle=\delta_{r s} 2 s\left(\sigma_{1}\right)_{\kappa \iota}, \quad\langle 1 / 2, \kappa, r|M| 1 / 2, \iota, s\rangle=\delta_{r s} \mu\left(\sigma_{3}\right)_{\kappa \iota} .
$$

Clearly, the tuple $(V, U, E)$ is irreducible, since the only operators commuting with $(V, U, E)$ are multiples of the identity.

$\operatorname{Put}\left(\Phi^{(\kappa, \iota)}\right)_{r s}:=\left\langle\frac{1}{2}, \kappa, r|\Phi| \frac{1}{2}, \iota, s\right\rangle$, where $\Phi(\mathbf{p}):=M+|\mathbf{p}| N$. It is

$$
\left(\begin{array}{ll}
\Phi^{(1,1)}(\mathbf{p}) & \Phi^{(1,2)}(\mathbf{p}) \\
\Phi^{(2,1)}(\mathbf{p}) & \Phi^{(2,2)}(\mathbf{p})
\end{array}\right)=\left(\begin{array}{cc}
\mu I_{2} & |\mathbf{p}| \sigma_{3} \\
|\mathbf{p}| \sigma_{3} & -\mu I_{2}
\end{array}\right)
$$

and $D^{(1 / 2)}(B)=B$ for all $B \in S U(2)$. Thus in the momentum space representation the energy operator is given by

$$
\left(\begin{array}{cc}
h^{(1,1)} & h^{(1,2)} \\
h^{(2,1)} & h^{(2,2)}
\end{array}\right)=\left(\begin{array}{cc}
B(\cdot) & 0 \\
0 & B(\cdot)
\end{array}\right)\left(\begin{array}{cc}
\Phi^{(1,1)} & \Phi^{(1,2)} \\
\Phi^{(2,1)} & \Phi^{(2,2)}
\end{array}\right)\left(\begin{array}{cc}
B(\cdot)^{-1} & 0 \\
0 & B(\cdot)^{-1}
\end{array}\right)=\left(\begin{array}{cc}
\mu I_{2} & \sigma(\cdot) \\
\sigma(\cdot) & -\mu I_{2}
\end{array}\right),
$$

where $\sigma(\mathbf{p}):=\sum_{i=1}^{3} p_{i} \sigma_{i}$. This proves (b).

It also follows (a), since the conditions of Theorem 5.3 hold. 
5.7 Definition. Let $\left(V^{D}, U^{D}, E^{D}\right)$ be the standard Dirac system with mass $\mu$ and let $1 \leq J \in \mathbb{N}_{0} / 2$. The Dirac tensor system with mass $\mu$ and spins $(J-1, J)$ is defined as $(V, U, E)$, where

$$
V(t):=V^{D}(t) \otimes I \quad U(\mathbf{b}, B):=U^{D}(\mathbf{b}, B) \otimes D^{(J-1 / 2)}(B) \quad E(\Delta):=E^{D}(\Delta) \otimes I
$$

for $t \in \mathbb{R},(\mathbf{b}, B) \in I S U(2)$ and $\Delta \in \mathscr{B}\left(\mathbb{R}^{3}\right)$. The energy operator for $V$ will be called the Dirac tensor operator.

In the next Theorem we will decompose the tensor product.

5.8 Theorem. Let $1 \leq J \in \mathbb{N}_{0} / 2$. The Dirac tensor system with mass $\mu$ and spins $(J-1, J)$ is a relativistic extendable causal localization and it is unitarily equivalent to

$$
\left(V, 2 U_{D^{(J-1)}} \oplus 2 U_{D^{(J)}}, E\right)
$$

where $E$ is the canonical projection-valued measure and the helicity representation of the energy operator of $V$ is the matrix multiplication operator corresponding to

$$
\langle k, \kappa, r|\Phi(\rho)| j, \iota, s\rangle=\delta_{r s}\left(\mu \delta_{k j}\left(\sigma_{3}\right)_{\kappa \iota}+\frac{\rho}{J}\left((-1)^{J-k} s \delta_{k j}+\sqrt{J^{2}-s^{2}} \delta_{|k-j|, 1}\right)\left(\sigma_{1}\right)_{\kappa \iota}\right),
$$

for $k, j \in\{J-1, J\}, r \in[k]$ and $s \in[j]$. Using Notation 5.5 we have

$$
\langle k, \kappa, r|\Phi(\rho)| j, \iota, s\rangle=\delta_{r s}\left(M_{s}^{(k, j)}+\rho N_{s}^{(k, j)}\right)_{\kappa \iota},
$$

where

$$
\begin{array}{ll}
N_{ \pm J}= \pm \sigma_{1}, & N_{s}=\frac{1}{J}\left(\begin{array}{cc}
s \sigma_{1} & \sqrt{(J-s)(J+s)} \sigma_{1} \\
\sqrt{(J-s)(J+s)} \sigma_{1} & -s \sigma_{1}
\end{array}\right) \\
M_{ \pm J}=\mu \sigma_{3}, & M_{s}=\left(\begin{array}{cc}
\mu \sigma_{3} & 0 \\
0 & \mu \sigma_{3}
\end{array}\right),
\end{array}
$$

for $|s| \neq J$.

Proof. Let $\left(V^{\prime}, U^{\prime}, E^{\prime}\right)$ be the Dirac tensor system with mass $\mu$ and spins $(J-1, J)$. By Theorem 4.9 we know that $\left(V^{\prime}, U^{\prime}, E^{\prime}\right)$ is a relativistic extendable causal localization. We note that the Dirac tensor operator is given by $H^{\prime}=H^{D} \otimes I$, where $H^{D}$ is the Dirac operator.

Let $T:\left(2 \mathbb{C}^{2}\right) \otimes \mathbb{C}^{2 J} \rightarrow 2\left(\mathbb{C}^{2 J-1} \oplus \mathbb{C}^{2 J+1}\right)$ be the unitary map which transforms the tensor product $\left(2 D^{(1 / 2)}\right) \otimes D^{(J-1 / 2)}$ into the direct sum $2\left(D^{(J-1)} \oplus D^{(J)}\right)$. Its inverse is given by

$$
T^{-1}|j, \iota, s\rangle:=\sum_{m} T_{m, s}^{(j)}\left|\frac{1}{2}, \iota, m\right\rangle \otimes\left|J-\frac{1}{2}, s-m\right\rangle
$$

where

$$
T_{m, s}^{(j)}:=(-1)^{1-J+s} \sqrt{2 j+1}\left(\begin{array}{ccc}
\frac{1}{2} & J-\frac{1}{2} & j \\
m & s-m & -s
\end{array}\right),
$$


see Appendix D. Let $S$ be the matrix multiplication operator corresponding to $T$. Then $S$ transforms $\left(U^{\prime}, E^{\prime}\right)$ into the standard form $(U, E)$, where $U:=2 U_{D^{(J-1)}} \oplus 2 U_{D^{(J)}}$. Let $V:=S V^{\prime} S^{-1}$ and let $H$ denote the energy operator for $V$. Since the helicity transform $X$ can be written as

$$
X[f]=\left[T\left(\left(2 D^{(1 / 2)}\right)\left(B(\cdot)^{-1}\right) \otimes D^{(J-1 / 2)}\left(B(\cdot)^{-1}\right)\right) T^{-1} f\right] \quad\left([f] \in L^{2}\right),
$$

we see that

$$
H^{h}=X \hat{H} X^{-1}=X S \hat{H}^{\prime} S^{-1} X^{-1}=S\left(\left(H^{D}\right)^{h} \otimes I\right) S^{-1},
$$

where $\left(H^{D}\right)^{h}$ is the helicity representation of the Dirac operator, i.e.

$$
\left(H^{D}\right)^{h}[f]=\left[\Phi^{D}(|\cdot|) f\right] \quad\left(f \in L^{2}\right)
$$

and $\Phi^{D}$ is given by Eq. (5.5). Hence $H^{h}[f]=[\Phi(|\cdot|) f]$, where

$$
\langle k, \kappa, r|\Phi| j, \iota, s\rangle=\sum_{m, m^{\prime}} T_{m, r}^{(k)} T_{m^{\prime}, s}^{(j)}\left\langle\frac{1}{2}, \kappa, m\left|\Phi^{D}\right| \frac{1}{2}, \iota, m^{\prime}\right\rangle \delta_{r-m, s-m^{\prime}}
$$

Put

$$
\left(\Phi_{m}^{D}\right)_{\kappa \iota}:=\left\langle\frac{1}{2}, \kappa, m\left|\Phi^{D}\right| \frac{1}{2}, \iota, m\right\rangle .
$$

Then, omitting the multiplicity indices $\kappa$ and $\iota$, we find

$$
\langle k, r|\Phi| j, s\rangle=\sum_{m, m^{\prime}} T_{m, r}^{(k)} T_{m^{\prime}, s}^{(j)} \delta_{m, m^{\prime}} \Phi_{m}^{D} \delta_{r-m, s-m^{\prime}}=\delta_{r s} \sum_{m} T_{m, s}^{(k)} T_{m, s}^{(j)} \Phi_{m}^{D} .
$$

Using

$$
\left(\begin{array}{ccc}
\frac{1}{2} & J-\frac{1}{2} & j \\
m & s-m & -s
\end{array}\right)=(-1)^{J-s+1}\left(\frac{J+(-1)^{J-j} 2 m s}{2 J(2 j+1)}\right)^{1 / 2} a_{m}^{(j)} \quad \forall s \in[j], m \in\left[\frac{1}{2}\right]
$$

where

$$
a_{m}^{(j)}:= \begin{cases}1, & j=J \\ 2 m, & j=J-1\end{cases}
$$

(cf. Lemma E.5) we obtain

$$
\langle k, r|\Phi| j, s\rangle=\delta_{r s} \frac{1}{2 J} \sum_{m}\left(\left(J+(-1)^{J-k} 2 m s\right)\left(J+(-1)^{J-j} 2 m s\right)\right)^{1 / 2} a_{m}^{(k)} a_{m}^{(j)} \Phi_{m}^{D} .
$$

If we write $\langle k, r|\Phi(\rho)| j, s\rangle=\delta_{r s}\left(M_{s}^{(k, j)}+\rho N_{s}^{(k, j)}\right)$ and use Notation 5.5 it is not difficult to find Eq. (5.7).

5.9 Lemma. Every Dirac tensor System is an irreducible relativistic extendable causal localization. 
Proof. Let $(V, U, E)$ be a Dirac tensor System. According to Theorem 5.8 we only need to show the irreducibility. In the helicity representation we may assume that the energy operator corresponds to $M+|\cdot| N$, where $M$ and $N$ are given by Eq. (5.7).

If $R^{h}$ is a bounded operator commuting with $\left(V^{h}, U^{h}, E^{h}\right)$ then $R^{h}$ is a matrix multiplication operator and by Notation 5.5 the corresponding matrix (denoted as $R$ ) is given by $R_{ \pm J}=S$ and

$$
R_{s}=\left(\begin{array}{cc}
S & 0 \\
0 & T
\end{array}\right), \quad \text { for } s \neq \pm J,
$$

for some matrices $S$ and $T$. Since $R^{h}$ commutes with $V^{h}$, it leaves $\mathscr{D}\left(H^{h}\right)$ invariant and $\left[H^{h}, R^{h}\right][f]=0$ for all $f \in \mathscr{D}(H)$. Therefore $[M+|\cdot| N, R]=0$ a.e. (note that $C_{c}\left(\mathbb{R}^{3}\right) \subset \mathscr{D}\left(H^{h}\right)$ and apply Lemma G.5). By continuity this holds everywhere, hence $[M, R]=[N, R]=0$. By means of Eq. (5.7) it is now easy to check that $R$ must be a multiple of the identity. Schur's Lemma (see Appendix I) then completes the proof.

5.10 Theorem. Every irreducible relativistic extendable causal localization is unitarily equivalent to the Dirac system or a Dirac tensor system.

Proof. Let $(V, U, E)$ be an irreducible relativistic extendable causal localization. By Corollary 4.4 and Lemma $4.5(V, U, E)$ contains a single mass $\mu>0$ and only bosons or fermions. Therefore, we may assume that $E$ is the canonical projection-valued measure and that

$$
U=\nu_{\ell} U_{D^{(\ell)}} \oplus \nu_{\ell+1} U_{D^{(\ell+1)}} \oplus \ldots \oplus \nu_{J} U_{D^{(J)}},
$$

where $\nu_{k} \in \mathbb{N}_{0}, J \in \mathbb{N}_{0} / 2$ and $\ell=0$ if $J \in \mathbb{N}$, else $\ell=1 / 2$.

If $J=0$ then Theorem 5.3 (d) and the selection rules of the Wigner $3 j$ symbols imply $N=0$, in contradiction to condition (b) of the same Theorem, i.e. $N^{2}=I$. If $J=1 / 2$ then Theorem 5.6 implies that $(V, U, E)$ is unitarily equivalent to the Dirac system. Thus let $J \geq 1$.

We use Notation 5.5. By Theorem 5.3 there are self-adjoint matrices $M$ and $N$ such that $V^{h}(t)[f]=\left[e^{i t(M+|\cdot| N)} f\right], N_{s}^{2}=I, N_{s} M_{s}+M_{s} N_{s}=0, M_{s}^{2}=\mu^{2} I$, and

$$
M_{s}=\operatorname{diag}\left(M^{(J)}, \ldots, M^{(|s|)}\right), \quad N_{s}^{(k, j)}=D_{s, 1}^{(k, j)} A^{(k, j)} .
$$

In the following we will introduce several unitary transforms commuting with $(U, E)$ to simplify the form of $M$ and $N$. After each step we will assume that the simplified form was given to begin with. Although being distinct transforms, we will denote them by the same letter $R$.

We have

$$
N_{J}=N_{J}^{(J, J)}, \quad M_{J}=M^{(J)} .
$$

Theorem 5.1 for $z \mapsto N_{J} z+M_{J}$ implies $\nu_{J}=2 \nu$ for some $\nu \in \mathbb{N}$ and there exists a unitary matrix $R^{(J)}$ such that

$$
R^{(J)} N_{J}^{(J, J)} R^{(J) *}=S_{1}:=\left(\begin{array}{cc}
0 & I_{\nu} \\
I_{\nu} & 0
\end{array}\right), \quad R^{(J)} M^{(J)} R^{(J) *}=\mu S_{3}:=\mu\left(\begin{array}{cc}
I_{\nu} & 0 \\
0 & -I_{\nu}
\end{array}\right) .
$$

\footnotetext{
${ }^{4}$ Here, $I_{a}$ for $a \in \mathbb{N}$ denotes the identity matrix acting on $\mathbb{C}^{a}$. However, sometimes, when there is no confusion possible, we will omit this subscript.
} 
Since the matrix multiplication operator corresponding to $R_{s}:=\operatorname{diag}\left(R^{(J)}, \ldots, R^{(|s|)}\right)$, where $R^{(k)}:=I$ for $k \neq J$, commutes with $(U, E)$, we may assume that $M^{(J)}$ and $N_{J}^{(J, J)}$ are already in this form.

The case $\nu_{J-1}=0$ can be excluded, otherwise we would have $N_{J-1}=N_{J-1}^{(J, J)}=$ $D_{J-1,1}^{(J, J)} A^{(J, J)}=\frac{D_{J-1,1}^{(J, J)}}{D_{J, 1}^{(J, J)}} N_{J}^{(J, J)}=\frac{J-1}{J} N_{J}$, but $N_{J-1}^{2}=I$ and $N_{J}^{2}=I$ implies $J=1 / 2$, which is impossible. Define $B \in \mathbb{C}^{\nu_{J} \times \nu_{J-1}}$ and $C \in \mathbb{C}^{\nu_{J-1} \times \nu_{J-1}}$ as

$$
N_{J-1}=\left(\begin{array}{cc}
N_{J-1}^{(J, J)} & N_{J-1}^{(J, J-1)} \\
N_{J-1}^{(J-1)} & N_{J-1}^{(J-1, J-1)}
\end{array}\right)=:\left(\begin{array}{cc}
\alpha S_{1} & B \\
B^{*} & C
\end{array}\right), \quad \alpha:=\frac{J-1}{J} .
$$

Since $C$ is self-adjoint, there exists a unitary matrix $R^{(J-1)}$ such that $R^{(J-1)} C R^{(J-1) *}$ is diagonal. Consider $R_{s}:=\operatorname{diag}\left(R^{(J)}, \ldots, R^{(|s|)}\right)$, where $R^{(k)}:=I$ for $k \neq J-1$. The transform corresponding to this matrix leaves $(U, E), M^{(J)}$ and $N_{J}^{(J, J)}$ unchanged, thus we may assume that $C$ is already diagonal.

Because

$$
\left(N_{J-1}\right)^{2}=\left(\begin{array}{cc}
\alpha S_{1} & B \\
B^{*} & C
\end{array}\right)\left(\begin{array}{cc}
\alpha S_{1} & B \\
B^{*} & C
\end{array}\right)=\left(\begin{array}{cc}
\alpha^{2} I_{\nu_{J}}+B B^{*} & \alpha S_{1} B+B C \\
a B^{*} S_{1}+C B^{*} & B^{*} B+C^{2}
\end{array}\right)=I_{\nu_{J}+\nu_{J+1}},
$$

we must have

$$
B B^{*}=\frac{2 J-1}{J^{2}} I_{\nu_{J}}
$$

Thus $B B^{*}$ is invertible and diagonal. This and the fact that $B \in \mathbb{C}^{\nu_{J} \times \nu_{J-1}}$ implies that $\nu_{J-1} \geq \nu_{J}$. Also, $B^{*} B$ is diagonal, since $B^{*} B=I-C^{2}$. The column vectors of $B$ are orthogonal and there are $\nu_{J-1}-\nu_{J}$ column vectors of $B$ which are zero. Let us interchange the column vectors via a permutation matrix $R^{(J-1)}$ such that

$$
B R^{(J-1) *}=\left(\begin{array}{ll}
B_{1} & 0
\end{array}\right) \quad B_{1} \in \mathbb{C}^{\nu_{J} \times \nu_{J}}, \quad 0 \in \mathbb{C}^{\nu_{J} \times\left(\nu_{J-1}-\nu_{J}\right)} .
$$

We note that $R^{(J-1)} C R^{(J-1) *}$ is still diagonal. Let

$$
\left(\begin{array}{cc}
C_{1} & 0 \\
0 & C_{2}
\end{array}\right):=R^{(J-1)} C R^{(J-1) *}, \quad C_{1} \in \mathbb{C}^{\nu_{J} \times \nu_{J}}, \quad C_{2} \in \mathbb{C}^{\left(\nu_{J-1}-\nu_{J}\right) \times\left(\nu_{J-1}-\nu_{J}\right)} .
$$

If $R_{s}:=\operatorname{diag}\left(R^{(J)}, \ldots, R^{(|s|)}\right)$, where $R^{(k)}:=I$ for $k \neq J-1$, then the transformation corresponding to this matrix leaves $(U, E), M^{(J)}$ and $N_{J}^{(J, J)}$ unchanged and

$$
\begin{aligned}
R_{J-1} N_{J-1} R_{J-1}^{*} & =\left(\begin{array}{cc}
I & 0 \\
0 & R^{(J-1)}
\end{array}\right)\left(\begin{array}{cc}
\alpha S_{1} & B \\
B^{*} & C
\end{array}\right)\left(\begin{array}{cc}
I & 0 \\
0 & R^{(J-1)^{*}}
\end{array}\right) \\
& =\left(\begin{array}{cc}
\alpha S_{1} & B R^{(J-1) *} \\
R^{(J-1)} B^{*} & R^{(J-1)} C R^{(J-1) *}
\end{array}\right)=\left(\begin{array}{ccc}
\alpha S_{1} & B_{1} & 0 \\
B_{1}^{*} & C_{1} & 0 \\
0 & 0 & C_{2}
\end{array}\right) .
\end{aligned}
$$


Therefore we can assume that $N_{J-1}$ is already in this form, where $C_{1}$ and $C_{2}$ are self-adjoint and diagonal. Again $\left(N_{J-1}\right)^{2}=I$ implies

$$
B_{1} B_{1}^{*}=\frac{2 J-1}{J^{2}} I, \quad \alpha S_{1} B_{1}+B_{1} C_{1}=0, \quad B_{1}^{*} B_{1}+C_{1}^{2}=I, \quad C_{2}^{2}=I,
$$

and since $B_{1}$ is a square matrix, we must have $B_{1}^{*} B_{1}=\frac{2 J-1}{J^{2}} I$. Hence $C_{1}^{2}=\alpha^{2} I$. For $M_{J-1}$ we have

$$
M_{J-1}=\left(\begin{array}{cc}
M^{(J)} & 0 \\
0 & M^{(J-1)}
\end{array}\right)=\left(\begin{array}{ccc}
\mu S_{3} & 0 & 0 \\
0 & M_{1} & M_{2} \\
0 & M_{2}^{*} & M_{4}
\end{array}\right),
$$

where $M_{1} \in \mathbb{C}^{\nu_{J} \times \nu_{J}}, M_{4} \in \mathbb{C}^{\left(\nu_{J-1}-\nu_{J}\right) \times\left(\nu_{J-1}-\nu_{J}\right)}$ are self-adjoint matrices and $M_{2}$ is a $\nu_{J} \times\left(\nu_{J-1}-\nu_{J}\right)$ matrix. Then $N_{J-1} M_{J-1}+M_{J-1} N_{J-1}=0$ implies

$$
\begin{gathered}
S_{1} S_{3}+S_{3} S_{1}=0, \quad B_{1} M_{1}+\mu S_{3} B_{1}=0, \quad B_{1} M_{2}=0, \\
B_{1}^{*} \mu S_{3}+M_{1} B_{1}^{*}=0, \quad C_{1} M_{1}+M_{1} C_{1}=0, \quad C_{1} M_{2}+M_{2} C_{2}=0, \\
M_{2}^{*} B_{1}^{*}=0, \quad C_{2} M_{2}^{*}+M_{2}^{*} C_{1}=0, \quad C_{2} M_{4}+M_{4} C_{2}=0 .
\end{gathered}
$$

But since $B_{1}$ is invertible, we must have $M_{2}=0$. From $M_{J-1}^{2}=\mu^{2} I$ we find $M_{1}^{2}=\mu^{2} I_{\nu_{J}}$ and $M_{4}^{2}=\mu^{2} I_{\nu_{J-1}-\nu_{J}}$.

By applying Theorem 5.1 for $z \mapsto-\left(C_{1} / \alpha\right) z+M_{1}$ we find that there exists a unitary matrix $R$ such that $R C_{1} R^{*}=-\alpha S_{1}$ and $R M_{1} R^{*}=\mu S_{3}$. Then

$$
R_{s}:=\operatorname{diag}\left(R^{(J)}, \ldots, R^{(|s|)}\right),
$$

where $R^{(k)}:=I$ for $k \neq J-1$ and

$$
R^{(J-1)}:=\operatorname{diag}(R, I),
$$

defines a unitary transform that leaves $(U, E), M^{(J)}$ and $N_{J}^{(J, J)}$ invariant. Therefore, we may assume that

$$
N_{J-1}=\left(\begin{array}{ccc}
\alpha S_{1} & B_{2} & 0 \\
B_{2}^{*} & -\alpha S_{1} & 0 \\
0 & 0 & C_{2}
\end{array}\right), \quad M_{J-1}=\left(\begin{array}{ccc}
\mu S_{3} & 0 & 0 \\
0 & \mu S_{3} & 0 \\
0 & 0 & M_{4}
\end{array}\right)
$$

Again $\left(N_{J-1}\right)^{2}=I_{\nu_{J}}$ and $N_{J-1} M_{J-1}+M_{J-1} N_{J-1}=0$ implies

$$
B_{2} B_{2}^{*}=\frac{2 J-1}{J^{2}} I, \quad S_{1} B_{2}-B_{2} S_{1}=0, \quad B_{2} S_{3}+S_{3} B_{2}=0 .
$$

Thus we must have

$$
B_{2}=\left(\begin{array}{ll}
0 & b \\
b & 0
\end{array}\right), \quad b b^{*}=\beta^{2} I_{\nu}
$$

where $b \in \mathbb{C}^{\nu \times \nu}$ and $\beta:=\frac{\sqrt{2 J-1}}{J}$. Put

$$
R:=\frac{1}{\beta}\left(\begin{array}{cc}
b & 0 \\
0 & b
\end{array}\right), \quad R^{(J-1)}:=\left(\begin{array}{cc}
R & 0 \\
0 & I
\end{array}\right),
$$


$R^{(k)}:=I$ for $k \neq J-1$ and $R_{s}:=\operatorname{diag}\left(R^{(J)}, \ldots, R^{(|s|)}\right)$. Then it is easy to see that $R$ is a unitary matrix commuting with $S_{1}$ and $S_{3}$. Thus the unitary transformation corresponding to $R_{s}$ leaves $(U, E), M_{J}, M_{J-1}$ and $N_{J}$ unchanged, and since $R^{*} B_{2}=$ $R B_{2}^{*}=\beta S_{1}$, we may assume that

$$
N_{J-1}=\left(\begin{array}{ccc}
\alpha S_{1} & \beta S_{1} & 0 \\
\beta S_{1} & -\alpha S_{1} & 0 \\
0 & 0 & C_{2}
\end{array}\right)
$$

In particular,

$$
N_{J-1}^{(J, J)}=\alpha S_{1}, \quad N_{J-1}^{(J, J-1)}=N_{J-1}^{(J-1, J)_{*}}=\left(\begin{array}{ll}
\beta S_{1} & 0
\end{array}\right), \quad N_{J-1}^{(J-1, J-1)}=\left(\begin{array}{cc}
-\alpha S_{1} & 0 \\
0 & C_{2}
\end{array}\right) .
$$

Now suppose $\nu_{J-2}>0$. This also means that $J \geq 2$. Because

$$
N_{s}^{(k, j)}=D_{s, 1}^{(k, j)} A^{(k, j)}
$$

and $D_{s, 1}^{(k, j)}=0$ for $|k-j|>1$, we have

$$
N_{J-2}=\left(\begin{array}{ccc}
N_{J-2}^{(J, J)} & N_{J-2}^{(J, 1)} & 0 \\
N_{J-2}^{(J-1, J)} & N_{J-1, J-1)}^{(J-2} & N_{J-2, J-2)}^{(J-1,2} \\
0 & N_{J-2}^{(J-2, J-1)} & N_{J-2}^{(J-2, J-2)}
\end{array}\right)
$$

The values of $D_{s, 1}^{(k, j)}$ can be calculated using E.1 and the symmetries of the Wigner $3 j$ symbol. Because

$$
N_{J-2}^{(k, j)}=\frac{D_{J-2,1}^{(k, j)}}{D_{J-1,1}^{(k, j)}} N_{J-1}^{(k, j)}, \quad \text { for } k, j \in\{J-1, J\}
$$

we find

$$
N_{J-2}=\left(\begin{array}{cccc}
\alpha^{\prime} S_{1} & \beta^{\prime} S_{1} & 0 & 0 \\
\beta^{\prime} S_{1} & -\alpha^{\prime} S_{1} & 0 & D_{1} \\
0 & 0 & \gamma^{\prime} C_{2} & D_{2} \\
0 & D_{1}^{*} & D_{2}^{*} & N_{J-2}^{(J-2, J-2)}
\end{array}\right)
$$

where

$$
\left(\begin{array}{c}
D_{1} \\
D_{2}
\end{array}\right):=N_{J-2}^{(J-1, J-2)}
$$

and

$$
\alpha^{\prime}:=\frac{J-2}{J}, \quad \beta^{\prime}:=2 \frac{J-1}{J}, \quad \gamma^{\prime}:=\frac{J-2}{J-1} .
$$

The condition $\left(N_{J-2}\right)^{2}=I$ implies $\beta^{\prime} S_{1} D_{1}=0$, hence $D_{1}=0$. Thus $N_{J-2}$ is block diagonal. Using Eq. (5.10) shows that this structure holds for all $N_{s},|s| \leq J-1$. So 
by the irreducibility we must have $\nu_{J}=\nu_{J-1}=2 \nu$ and $\nu_{J-2}=\ldots=\nu_{\ell}=0$. Moreover, if $\nu>1$ then $(V, U, E)$ is reducible, since

$$
P_{ \pm J}:=T, \quad P_{s}:=\operatorname{diag}(T, T) \text { for }|s| \neq J, \quad T_{\kappa \iota}:=\delta_{\kappa \iota}\left(\delta_{\kappa, 1}+\delta_{\kappa, \nu+1}\right)
$$

defines an orthogonal projection onto an invariant closed subspace for $(V, U, E)$. Therefore it is $\nu_{J}=\nu_{J-1}=2$.

So far we have

$$
N_{J}=\sigma_{1}, \quad N_{J-1}=\left(\begin{array}{cc}
\alpha \sigma_{1} & \beta \sigma_{1} \\
\beta \sigma_{1} & -\alpha \sigma_{1}
\end{array}\right), \quad M_{J}=\mu \sigma_{3}, \quad M_{J-1}=\left(\begin{array}{cc}
\mu \sigma_{3} & 0 \\
0 & \mu \sigma_{3}
\end{array}\right) .
$$

Note that $N_{s}^{(k, j)}=D_{s, 1}^{(k, j)} A^{(k, j)}$ is satisfied by

$$
\begin{gathered}
A^{(J, J)}=\frac{(-1)^{2 J+1}}{\sqrt{3}} \sqrt{\frac{(J+1)(2 J+1)}{J}} \sigma_{1}, \quad A^{(J, J-1)}=\frac{(-1)^{2 J+1}}{\sqrt{3}} \sqrt{\frac{(2 J+1)(2 J-1)}{J}} \sigma_{1} \\
A^{(J-1, J)}=-A^{(J, J-1)}, \quad A^{(J-1, J-1)}=-\frac{(-1)^{2 J+1}}{\sqrt{3}} \sqrt{\frac{(J-1)(2 J-1)}{J}} \sigma_{1} .
\end{gathered}
$$

(Although $D_{s, 1}^{(J-1, J-1)}=0$ for $J=1$ this is not a problem to find a suitable $A^{(J-1, J-1)}$ since in this case it is arbitrary.) Hence

$$
\begin{gathered}
N_{s}^{(J, J)}=\frac{s}{J} \sigma_{1}, \quad N_{s}^{(J, J-1)}=\frac{\sqrt{(J-s)(J+s)}}{J} \sigma_{1} \\
N_{s}^{(J-1, J)}=\frac{\sqrt{(J-s)(J+s)}}{J} \sigma_{1}, \quad N_{s}^{(J-1, J-1)}=-\frac{s}{J} \sigma_{1},
\end{gathered}
$$

and

$$
M_{s}^{(k, j)}=\delta_{k j} \mu \sigma_{3}
$$

Now Theorem 5.8 shows that $(V, U, E)$ is unitarily equivalent to the Dirac tensor system with mass $\mu$ and spins $(J-1, J)$.

5.11 Corollary. Every relativistic extendable causal localization is unitarily equivalent to a direct sum of Dirac systems and/or Dirac tensor systems.

Proof. Let $(V, U, E)$ be a relativistic extendable causal localization and let $(W, E)$ be an relativistic extension of $(V, U, E)$. Then $(W, E)$ decomposes as a direct sum of a Boson and Fermion systems, and each further decomposes into a direct sum of single mass systems. Plainly, $(V, U, E)$ decomposes in the same way and these components are relativistic extendable causal localizations. So we may assume that $(V, U, E)$ contains a single mass $\mu>0$ and only bosons or fermions.

Now start with the highest spin $J$ occurring in $U$. If $J=1 / 2$, then the proof of Theorem 5.6 shows that $(V, U, E)$ is a direct sum of Dirac systems. If $J>1 / 2$, then the proof of Theorem 5.10 shows that $(V, U, E)$ contains Dirac tensor systems with spins $(J-1, J)$. After separating these systems one repeats the above steps. 
5.12. The Dirac tensor system in the coordinate space representation. Let $(V, U, E)$ be the Dirac tensor system with mass $\mu$ and spins $(J-1, J)$. The helicity representation of the Dirac tensor operator $H$ was given in Theorem 5.8. By means of Eq. (5.8) it was not necessary to calculate $H$ in the momentum or coordinate space representation. So let us catch up on this.

In the coordinate space representation $U$ has the form

$$
U=2 U_{D^{(J-1)}} \oplus 2 U_{D^{(J)}}
$$

and $E$ is the canonical projection-valued measure. The form of $H$ is more complex: We note that in the momentum space representation $\hat{H}[f]=[h f]$, where

$$
\langle k, \kappa, r|h| j, \iota, s\rangle=\sum_{m, m^{\prime}} T_{m, r}^{(k)} T_{m^{\prime}, s}^{(j)}\left\langle\frac{1}{2}, \kappa, m\left|h^{D}\right| \frac{1}{2}, \iota, m^{\prime}\right\rangle \delta_{r-m, s-m^{\prime}}
$$

and $h^{D}$ is given by Eq. (5.4) (see the proof of Theorem 5.8). The block matrix structure of $h$ is given by

$$
h=\left(\begin{array}{llll}
h_{11}^{(J-1, J-1)} & h_{12}^{(J-1, J-1)} & h_{11}^{(J-1, J)} & h_{12}^{(J-1, J)} \\
h_{21}^{(J-1, J-1)} & h_{22}^{(J-1, J-1)} & h_{21}^{(J-1, J)} & h_{22}^{(J-1, J)} \\
h_{11}^{(J, J-1)} & h_{12}^{(J, J-1)} & h_{11}^{(J, J)} & h_{12}^{(J, J)} \\
h_{21}^{(J, J-1)} & h_{22}^{(J, J-1)} & h_{21}^{(J, J)} & h_{22}^{(J, J)}
\end{array}\right),
$$

where

$$
\left(h_{\kappa \iota}^{(k, j)}\right)_{r s}:=\langle k, \kappa, r|h| j, \iota, s\rangle
$$

We then obtain

$$
H=\left(\begin{array}{cccc}
\mu I_{2 J-1} & a & 0 & b \\
a & -\mu I_{2 J-1} & b & 0 \\
0 & b^{*} & \mu I_{2 J+1} & c \\
b^{*} & 0 & c & -\mu I_{2 J+1}
\end{array}\right)
$$

where

$$
a:=\sum_{k=1}^{3} a_{k}\left(-i \partial_{k}\right), \quad b:=\sum_{k=1}^{3} b_{k}\left(-i \partial_{k}\right), \quad c:=\sum_{k=1}^{3} c_{k}\left(-i \partial_{k}\right)
$$

and

$$
\begin{aligned}
& \left(a_{1}\right)_{r s}:=\delta_{r, s-1} \phi_{r, s}^{(+,-)}+\delta_{r, s+1} \phi_{r, s}^{(-,+)} \quad\left(a_{2}\right)_{r s}:=-i \delta_{r, s-1} \phi_{r, s}^{(+,-)}+i \delta_{r, s+1} \phi_{r, s}^{(-,+)} \\
& \left(a_{3}\right)_{r s}:=\delta_{r, s}\left(\phi_{r, s}^{(+,+)}-\phi_{r, s}^{(-,-)}\right) \\
& \left(b_{1}\right)_{r s}:=-\delta_{r, s-1} \phi_{r, s}^{(+,+)}+\delta_{r, s+1} \phi_{r, s}^{(-,-)} \quad\left(b_{2}\right)_{r s}:=i \delta_{r, s-1} \phi_{r, s}^{(+,+)}+i \delta_{r, s+1} \phi_{r, s}^{(-,-)} \\
& \left(b_{3}\right)_{r s}:=-\delta_{r, s}\left(\phi_{r, s}^{(+,-)}+\phi_{r, s}^{(-,+)}\right) \\
& \left(c_{1}\right)_{r s}:=\delta_{r, s-1} \phi_{r, s}^{(-,+)}+\delta_{r, s+1} \phi_{r, s}^{(+,-)} \quad\left(c_{2}\right)_{r s}:=-i \delta_{r, s-1} \phi_{r, s}^{(-,+)}+i \delta_{r, s+1} \phi_{r, s}^{(+,-)} \\
& \left(c_{3}\right)_{r s}:=-\delta_{r, s}\left(\phi_{r, s}^{(+,+)}-\phi_{r, s}^{(-,-)}\right)
\end{aligned}
$$


where

$$
\phi_{r s}^{(u, v)}:=\frac{\sqrt{(J+u r)(J+v s)}}{2 J} .
$$

By interchanging the second row of blocks with the third row and the second column with the third column we obtain

$$
H \rightarrow\left(\begin{array}{cc}
\mu I & S \\
S^{*} & -\mu I
\end{array}\right)
$$

where $I$ is the identity matrix in $\mathbb{C}^{2(J-1)+1} \oplus \mathbb{C}^{(2 J+1)}$ and

$$
S:=\sum_{k=1}^{3} S_{k}\left(-i \partial_{k}\right), \quad S_{k}:=\left(\begin{array}{ll}
a_{k} & b_{k} \\
b_{k}^{*} & c_{k}
\end{array}\right)
$$

In this modified coordinate space representation $U$ becomes

$$
U \rightarrow 2\left(U_{D^{(J-1)}} \oplus U_{D^{(J)}}\right) .
$$

The similarity to the Dirac system now is obvious.

5.13 Discussion. In the Schrödinger picture states become time depending, i.e. for $\hat{\psi}_{0} \in \mathscr{D}(\hat{H})$ one has

$$
\hat{\psi}(t):=e^{-i t \hat{H}} \hat{\psi}_{0}, \quad t \in \mathbb{R} .
$$

Then $\hat{\psi}$ satisfies Schrödinger's equation $i \partial_{t} \hat{\psi}=\hat{H} \hat{\psi}$. For the Dirac operator we obtain in the coordinate space representation

$$
H^{D}=\mu\left(\begin{array}{cc}
I_{2} & 0 \\
0 & -I_{2}
\end{array}\right)+\sum_{k=1}^{3}\left(\begin{array}{cc}
0 & \sigma_{k} \\
\sigma_{k} & 0
\end{array}\right)\left(-i \partial_{k}\right)
$$

So the Dirac equation is Schrödinger's equation for the Dirac operator. Since the Dirac tensor operator is $H=H^{D} \otimes I$, there is a unitary transformation $S$ such that $S H S^{-1}$ is a direct sum of Dirac operators, and we see that Schrödinger's equation for a relativistic extendable causal localization is always unitarily equivalent to a direct sum of Dirac equations. But the same does not hold for the $I S U(2)$ representation $U$ of the Dirac tensor system $(V, U, E)$, where $U=U^{D} \otimes D^{J-1}$. Clearly, $S U S^{-1}$ is not a direct sum of $U_{D^{(1 / 2)}}$, since the Dirac tensor system is irreducible.

5.14. Higher dimensional Dirac equations. The Dirac tensor operator is

$$
H=\sum_{k=1}^{3} \alpha_{n, k}\left(-i \partial_{k}\right)+\beta_{n} \mu
$$

with

$$
\alpha_{n, k}=\left(\begin{array}{cc}
0 & \sigma_{k} \\
\sigma_{k} & 0
\end{array}\right) \otimes I_{n}, \quad \beta_{n}=\left(\begin{array}{cc}
I_{2} & 0 \\
0 & -I_{2}
\end{array}\right) \otimes I_{n}, \quad n \in \mathbb{N}
$$


For $n=1$ we obtain the Dirac operator. The rotations are represented as

$$
(U(B) g)(\mathbf{x})=\left(\operatorname{diag}(B, B) \otimes D^{(n / 2-1 / 2)}(B)\right) g\left(B^{-1} \mathbf{x}\right) .
$$

It is clear that $\alpha_{n, k}$ and $\beta_{n}$ satisfy the known anti-commutation relations

$$
\left\{\alpha_{n, j}, \alpha_{n, k}\right\}=2 \delta_{j k} I, \quad\left\{\alpha_{n, j}, \beta_{n}\right\}=0, \quad \alpha_{n, j}^{2}=\beta_{n}^{2}=I .
$$

The momentum operator $\mathbf{P}$ is given by $P_{j}=-i \partial_{j}$ and the causal position operator $\mathbf{X}^{c}$ is the multiplication with $\mathbf{x}$. Every irreducible relativistic extendable causal localization is up to unitary equivalence of the above form. For every $n \in \mathbb{N}$ there is a Dirac equation for mass $\mu$ and spin spectrum $\{n / 2-1, n / 2\}$ for $n>1$ and $\{1 / 2\}$ for $n=1$.

Similarly to Dirac's ansatz the anti-commutation relations are necessary to obtain a relativistic description. However, Dirac chose a linear equation in order to obtain a positive definite density, in our approach the linearity is a consequence of the causality condition.

In the next section we will see that the boosts for these systems are given by

$$
\mathbf{N}:=\frac{1}{2}\left\{H, \mathbf{X}^{c}\right\}+\frac{\operatorname{sgn}(H)}{|H|+\mu} \mathbf{P} \times\left(\mathbf{S}^{c}-\frac{1}{4 i} \mathbf{A} \times \mathbf{A}\right), \quad \mathbf{A}:=-i\left[\mathbf{X}^{c}, H\right], \quad \mathbf{S}^{c}:=\mathbf{J}-\mathbf{X}^{c} \times \mathbf{P} .
$$




\section{Relativistic Extensions for the Dirac Systems}

In section 5 we have seen that the Dirac system and the Dirac tensor system are elementary building blocks of every relativistic extendable causal localization. Using a Foldy-Wouthuysen transformation and the BTF formula (3.5) we can determine their relativistic extensions.

6.1 Theorem. Let $(V, U, E)$ be the Dirac system. Then there exists a unique representation $W$ of $I S L(2, \mathbb{C})$ extending $(V, U)$ such that the boosts for $W$ are given by

$$
\mathbf{N} \psi=\frac{1}{2}\left(H \mathbf{X}^{c}+\mathbf{X}^{c} H\right) \psi \quad \forall \psi \in \mathscr{D},
$$

where $\mathbf{X}^{c}$ is the position operator corresponding to $E$ and $\mathscr{D}$ is a dense subspace satisfying $\mathscr{D} \subset \mathscr{D}(A), A \mathscr{D} \subset \mathscr{D}$ and $\overline{\left.A\right|_{\mathscr{D}}}=A$ for every $A \in\left\{\mathbf{X}^{c}, \mathbf{X}, H, \mathbf{P}, \mathbf{J}, \mathbf{N}\right\}$ (cf. Theorem 3.10 (a)). Here, $\mathbf{X}$ denotes the Newton-Wigner position operator for $W$. Moreover, $W$ is a finite massive representation.

Proof. We may assume that the Dirac system is given in standard form. Throughout this proof we will work in the momentum space representation so there will be no need to use the hat notation.

The Dirac operator corresponds to the matrix-valued function $h: \mathbb{R}^{3} \rightarrow \mathbb{C}^{4 \times 4}$, which can be written as $h(\mathbf{p})=M+K(\mathbf{p})$, where

$$
M=\left(\begin{array}{cc}
\mu I_{2} & 0 \\
0 & -\mu I_{2}
\end{array}\right), \quad K(\mathbf{p})=\left(\begin{array}{cc}
0 & \sigma(\mathbf{p}) \\
\sigma(\mathbf{p}) & 0
\end{array}\right)
$$

(cf. Eq. (5.4)). In this form it is easy to see that the matrix multiplication operator corresponding to $M$ commutes with $(U, E)$. Moreover, we have $M^{2}=\mu^{2} I_{4}, M K(\mathbf{p})=$ $-K(\mathbf{p}) M$ and $K(\mathbf{p})^{2}=\mathbf{p}^{2}$.

Since $U=U_{D^{(1 / 2)}} \oplus U_{D^{(1 / 2)}}$, it is clear that $W^{\prime}:=W^{(\mu,+1,1 / 2)} \oplus W^{(\mu,-1,1 / 2)}$ is a finite massive representation of $I S L(2, \mathbb{C})$ extending $U$ - here we need a positive and a negative energy representation, because $h$ has positive and negative eigenvalues. But this is not an extension for $V$, since the energy operator for $W^{\prime}$ is diagonal and $H$ is not. We thus need to diagonalize $H$ by means of a unitary operator that commutes with $U$. To this end consider the matrix-valued function $Y: \mathbb{R}^{3} \rightarrow \mathbb{C}^{4 \times 4}$,

$$
Y:=\frac{1}{\sqrt{2 \epsilon(\epsilon+\mu)}}\left(\frac{\epsilon+\mu}{\mu} M+K\right)
$$

where $\epsilon(\mathbf{p}):=\sqrt{\mu^{2}+\mathbf{p}^{2}}$. It is easy to check that $Y(\mathbf{p})=Y(\mathbf{p})^{*}=Y(\mathbf{p})^{-1}$. Because $[H, U] \psi=0$ for all $\psi \in C_{c}$, it is clear that the matrix multiplication operator corresponding to $K$ commutes with $U$ on $C_{c}$. Hence, the matrix multiplication operator $Y_{\text {op. }}$. 
corresponding to $Y$, commutes with $U$ on $C_{c}$. Since $Y_{\text {op. }}$ is bounded and $C_{c}$ is dense in $L^{2}, Y_{\text {op. }}$ commutes with $U$. Also,

$$
\begin{aligned}
Y h Y^{-1} & =\frac{1}{2 \epsilon(\epsilon+\mu)}\left(\frac{\epsilon}{\mu} M+h\right) h\left(\frac{\epsilon}{\mu} M+h\right) \\
& =\frac{1}{2 \epsilon(\epsilon+\mu)}\left(\frac{\epsilon^{2}}{\mu^{2}}\left(\mu^{2} M-\mu^{2} K\right)+2 \frac{\epsilon}{\mu} \epsilon^{2} M+\epsilon^{2} M+\epsilon^{2} K\right)=\frac{\epsilon}{\mu} M .
\end{aligned}
$$

Hence $W:=Y_{\mathrm{op}}^{-1} W^{\prime} Y_{\mathrm{op}}$. is an extension for $(V, U)$. Since $Y_{\mathrm{op}} C_{c}^{\infty}=C_{c}^{\infty}$, the proof of Theorem 3.10 shows that $C_{c}^{\infty}$ is a common core for $\mathbf{X}, \mathbf{X}^{c}, H, \mathbf{P}, \mathbf{J}, \mathbf{N}$ and each of these operators leaves $C_{c}^{\infty}$ invariant.

For the remaining part of the proof it is understood that all equations hold within $C_{c}^{\infty}$. Moreover, we will not introduce new symbols to distinguish between a matrix multiplication operator and its corresponding matrix-valued function. From the context it should be clear which object we consider.

The Newton-Wigner position operator for $W^{\prime}$ in the momentum space representation is $X_{j}^{c}=i \partial_{j}$. Thus the Newton-Wigner position operator for $W$ is given by

$$
X_{j}=Y^{-1} X_{j}^{c} Y=X_{j}^{c}+Y^{-1}\left(i \partial_{j} Y\right)=: X_{j}^{c}+F_{j} .
$$

By means of the BTF formula (3.5) we can compute the boost $\mathbf{N}$ for $W$. The spin vector for $(U, E)$ in the momentum space representation is given by (see Lemma 1.16)

$$
S_{k}^{c}=\left(\mathbf{J}-\mathbf{X}^{c} \times \mathbf{P}\right)_{k}=\frac{1}{2}\left(\begin{array}{cc}
\sigma_{k} & 0 \\
0 & \sigma_{k}
\end{array}\right) .
$$

If we define

$$
A_{k}:=\left(\begin{array}{cc}
0 & \sigma_{k} \\
\sigma_{k} & 0
\end{array}\right)
$$

we may write $K=\mathbf{P} \cdot \mathbf{A}$ and

$$
\mathbf{P} \times \mathbf{S}^{c}=\frac{1}{2 i}(\mathbf{P}-K \mathbf{A}) .
$$

This implies $K \mathbf{A}=\mathbf{P}-2 i \mathbf{P} \times \mathbf{S}^{c}$. Taking the adjoint of this equation shows that

$$
[\mathbf{A}, K]=4 i \mathbf{P} \times \mathbf{S}^{c}=2(\mathbf{P}-K \mathbf{A}) .
$$

Since $\mathbf{S}=\mathbf{J}-\left(\mathbf{X}^{c}+\mathbf{F}\right) \times \mathbf{P}=\mathbf{S}^{c}-\mathbf{F} \times \mathbf{P}$, we have to calculate

$$
\mathbf{N}=\frac{1}{2}\left\{H, \mathbf{X}^{c}\right\}+\frac{1}{2}\{H, \mathbf{F}\}+H \frac{1}{\epsilon(\epsilon+\mu)}\left(\mathbf{P} \times\left(\mathbf{S}^{c}-\mathbf{F} \times \mathbf{P}\right)\right),
$$

where $\{A, B\}:=A B+B A$. It is

$$
\mathbf{F}=\frac{i}{2 \epsilon}\left(\frac{1}{\mu} M+\frac{1}{\epsilon+\mu} K\right) \mathbf{A}+\frac{i}{2 \epsilon(\epsilon+\mu)}\left(\frac{1}{\epsilon \mu} K M-1\right) \mathbf{P} .
$$


Using $M K=-K M, M \mathbf{A}=-\mathbf{A} M, M^{2}=\mu^{2}, K^{2}=\mathbf{P}^{2}$ and the commutation relation for $\mathbf{A}$ and $K$ we find

$$
\{H, M \mathbf{A}\}=2 M(\mathbf{P}-K \mathbf{A}), \quad\{H, K \mathbf{A}\}=2 M K \mathbf{A}+2 K \mathbf{P}, \quad\{H, K M\}=0 .
$$

Thus

$$
\frac{1}{2}\{H, \mathbf{F}\}=\frac{i}{2 \mu(\epsilon+\mu)} M(\mathbf{P}-K \mathbf{A})
$$

By means of

$$
\begin{aligned}
\mathbf{P} \times(\mathbf{F} \times \mathbf{P}) & =\frac{i}{2 \epsilon}\left(\frac{1}{\mu} M+\frac{1}{\epsilon+\mu} K\right) \mathbf{P} \times(\mathbf{A} \times \mathbf{P}) \\
& =\frac{i}{2 \epsilon}\left(\frac{1}{\mu} \mathbf{P}^{2} M \mathbf{A}-\frac{1}{\mu} M K \mathbf{P}+\frac{1}{\epsilon+\mu} \mathbf{P}^{2}(K \mathbf{A}-\mathbf{P})\right)
\end{aligned}
$$

we obtain

$$
\begin{aligned}
\mathbf{P} \times \mathbf{S}^{c}-\mathbf{P} \times(\mathbf{F} \times \mathbf{P}) & =-\frac{i}{2 \epsilon \mu}\left(\left(\mu^{2}-M K\right) \mathbf{P}+\left(\mathbf{P}^{2} M-\mu^{2} K\right) \mathbf{A}\right) \\
& =-\frac{i}{2 \epsilon \mu}\left(H M \mathbf{P}+\left(\mathbf{P}^{2} M-\mu^{2} K\right) \mathbf{A}\right) \\
& =-\frac{i}{2 \epsilon \mu} H M(\mathbf{P}-K \mathbf{A}) .
\end{aligned}
$$

Hence $\mathbf{N}=\left\{H, \mathbf{X}^{c}\right\} / 2$.

6.2 Note. The unitary transformation $Y$ given by $(6.2)$ is similar to a Foldy-Wouthuysen transformation [FW50], [[Tha92] Sec. 1.4.3.]. There are many unitary $Y$ which diagonalize $H$ and commute with $U$. Some of these yield the same $\mathbf{N}$ but some yield different $\mathbf{N}$, e.g., consider $e^{i \epsilon / \mu} Y$ instead of $Y$. This means that there are different finite massive relativistic extensions, say $W$ and $W^{\prime}$, for the Dirac system. Although $W$ and $W^{\prime}$ are unitarily equivalent, $(W, E)$ and $\left(W^{\prime}, E\right)$ are not, which is easy to see, since the Dirac system is irreducible. But if there are different extensions for the Dirac system, then which extension describes the electron? At the time of this writing we can give no satisfactory answer. However, good reasons to choose $\mathbf{N}=\left\{H, \mathbf{X}^{c}\right\} / 2$ is its simplicity and the fact that this expression is also obtained by symmetrizing the classical expression $\mathbf{x} H$ for $\mathbf{N}$ (see [[Pry48 Eq. (6.5)] and [[Tha92] Eq. (1.39) and (2.71)]).

Similarly, it is not possible to talk about a unique Newton-Wigner localization when considering the Dirac system, at least by our definition: By the BTF formula the Newton-Wigner localization is uniquely defined for every massive relativistic representation. For the Dirac system there exist different massive relativistic extensions. Consequently there are different Newton-Wigner localization for the Dirac system.

6.3 Theorem. Let $(V, U, E)$ be a Dirac tensor system. Then there exists a unique representation $W$ of $I S L(2, \mathbb{C})$ extending $(V, U)$ such that the boosts for $W$ are given by

$$
\mathbf{N} \psi=\left(\frac{1}{2}\left\{H, \mathbf{X}^{c}\right\}+\frac{\operatorname{sgn}(H)}{|H|+\mu} \mathbf{P} \times\left(\mathbf{S}^{c}-\frac{1}{4 i} \mathbf{A} \times \mathbf{A}\right)\right) \psi \quad \forall \psi \in \mathscr{D},
$$


where $\mathbf{X}^{c}$ is the position operator corresponding to $E, \mathbf{A}:=-i\left[\mathbf{X}^{c}, H\right], \mathbf{S}^{c}=\mathbf{J}-\mathbf{X}^{c} \times \mathbf{P}$ is the spin vector of $(U, E)$ and $\mathscr{D} i s$ a dense subspace satisfying $\mathscr{D} \subset \mathscr{D}(A), A \mathscr{D} \subset \mathscr{D}$ and $\overline{\left.A\right|_{\mathscr{D}}}=A$ for every $A \in\left\{\mathbf{X}^{c}, \mathbf{X}, H, \mathbf{P}, \mathbf{J}, \mathbf{N}\right\}$ (cf. Theorem 3.10 (a)). Here, $\mathbf{X}$ denotes the Newton-Wigner position operator for $W$. Moreover, $W$ is a finite massive representation.

Proof. We declare the same conventions as in the proof of Theorem 6.1.

Since $(V, U, E)$ is a Dirac tensor system, there exists a $J \geq 1$ such that $U(\mathbf{b}, B)=$ $U^{D}(\mathbf{b}, B) \otimes D^{(J-1 / 2)}(B)$ for every $(\mathbf{b}, B) \in I S U(2)$. Let $\mathbf{L}$ denote the generator of the $S U(2)$ representation $D^{(J-1 / 2)}$. Then the generators for the Dirac tensor system are given by

$$
H=H^{D} \otimes I_{2 J}, \quad \mathbf{P}=\mathbf{P}^{D} \otimes I_{2 J}, \quad \mathbf{J}=\mathbf{J}^{D} \otimes I_{2 J}+I \otimes \mathbf{L}, \quad \mathbf{X}^{c}=\mathbf{X}^{D} \otimes I_{2 J},
$$

where $H^{D}, \mathbf{P}^{D}$ and $\mathbf{J}^{D}$ are the generators of the Dirac system and $\mathbf{X}^{D}$ is the position operator corresponding to the projection-valued measure of the Dirac system.

Since $H^{D}$ can be written as $H^{D}=M^{D}+\mathbf{P}^{D} \cdot \mathbf{A}^{D}$, we have $H=M+\mathbf{P} \cdot \mathbf{A}$, where $M:=M^{D} \otimes I_{2 J}$ and $\mathbf{A}:=\mathbf{A}^{D} \otimes I_{2 J}$. Thus $\mathbf{A}=-i\left[\mathbf{X}^{c}, H\right]$.

Put $Y:=Y^{D} \otimes I_{2 J}$, where $Y^{D}$ is the Foldy-Wouthuysen transformation defined in Eq. 6.2). Then $Y$ commutes with $U$ and diagonalizes the Dirac tensor operator. Thus a Newton-Wigner position operator for $(V, U, E)$ is given by

$$
\mathbf{X}:=Y^{-1} \mathbf{X}^{c} Y=\mathbf{X}^{c}+\mathbf{F} \otimes I_{2 J} .
$$

Then the BTF formula for $\mathbf{N}$ gives

$$
\mathbf{N}=\frac{1}{2}\left\{H, \mathbf{X}^{c}\right\}+\frac{\operatorname{sgn}(H)}{|H|+\mu} \mathbf{P} \times(I \otimes \mathbf{L}) .
$$

Note that

$$
\begin{aligned}
I \otimes \mathbf{L} & =\mathbf{J}-\mathbf{J}^{D} \otimes I_{2 J}=\mathbf{J}-\left(\mathbf{S}^{D} \otimes I_{2 J}+\left(\mathbf{X}^{D} \times \mathbf{P}^{D}\right) \otimes I_{2 J}\right) \\
& =\mathbf{J}-\mathbf{X}^{c} \times \mathbf{P}-\mathbf{S}^{D} \otimes I_{2 J}=\mathbf{S}^{c}-\mathbf{S}^{D} \otimes I_{2 J},
\end{aligned}
$$

where $\mathbf{S}^{c}=\mathbf{J}-\mathbf{X}^{c} \times \mathbf{P}$ is the spin vector of $(U, E)$. Since

$$
\mathbf{S}^{D}=\frac{1}{4 i} \mathbf{A}^{D} \times \mathbf{A}^{D}
$$

(cf. [[Tha92] Eq. (1.152)]), we have

$$
I \otimes \mathbf{L}=\mathbf{S}^{c}-\frac{1}{4 i} \mathbf{A} \times \mathbf{A} .
$$

Hence

$$
\mathbf{N}=\frac{1}{2}\left\{H, \mathbf{X}^{c}\right\}+\frac{\operatorname{sgn}(H)}{|H|+\mu} \mathbf{P} \times\left(\mathbf{S}^{c}-\frac{1}{4 i} \mathbf{A} \times \mathbf{A}\right)
$$

and the proof is complete. 
6.4 Discussion. Let $(V, U, E)$ be a finite causal localization. By Lemma 4.7 we have necessary and sufficient conditions for the existence of a finite massive representation extending $(V, U)$. If the existence of such an extension is given then the boosts

$$
\mathbf{N}:=\frac{1}{2}\left\{H, \mathbf{X}^{c}\right\}+\frac{\operatorname{sgn}(H)}{|H|+C^{1 / 2}} \mathbf{P} \times\left(\mathbf{S}^{c}-\frac{1}{4 i} \mathbf{A} \times \mathbf{A}\right), \quad \mathbf{A}:=-i\left[\mathbf{X}^{c}, H\right]
$$

define such an extension. Note that for the Dirac system

$$
\mathbf{S}^{c}-\frac{1}{4 i} \mathbf{A} \times \mathbf{A}=0 .
$$

Thus this extension holds for finite direct sums of Dirac systems and Dirac tensor systems, and since these are the only irreducible finite causal localization, it holds for every finite causal localization which has an extension. We summarize this in the following Corollary.

6.5 Corollary. Let $(V, U, E)$ be a finite causal localization and let $\mathbf{X}^{c}$ be the position operator corresponding to E. Suppose the following conditions hold.

(i) There exists a bounded operator $C$ having finite positive spectrum such that $H^{2}=$ $C+\mathbf{P}^{2}$

(ii) There exists a projection-valued measure $F: \mathscr{B}\left(\mathbb{R}^{3}\right) \rightarrow L(\mathscr{H})$ such that $(U, F)$ is a localization and $[\operatorname{sgn} H, F]=[C, F]=0$.

Then the boosts in Eq. (6.4) define a finite massive relativistic extension for $(V, U, E)$. 


\section{Non-Relativistic Causal Localizations with a Sin- gle Spin of Multiplicity One}

Causal localizations with a single spin of multiplicity one, i.e. $\nu_{k}=\delta_{j k}$, are studied. We already know that these do not have finite massive relativistic extensions. But we think it is worthwhile to see that there are causal localizations besides the Dirac- and the Dirac tensor-system.

7.1 Theorem. Let $U:=U_{D^{(j)}}$, let $E$ be the canonical projection-valued measure and let $V$ be a time evolution on $L^{2}\left(\mathbb{R}^{3}, \mathbb{C}^{2 j+1}\right)$. Then $(V, U, E)$ is a causal localization if and only if in the helicity representation $V^{h}(t)[f]=\left[\Phi_{t}(|\cdot|) f\right]$ the matrix-valued function $\Phi_{t}: \mathbb{R}_{\geq 0} \rightarrow \mathbb{C}^{2 j+1}$ satisfies

$$
\left\langle j, r\left|\Phi_{t}(\rho)\right| j, s\right\rangle=\delta_{r s} e^{i t(c+a s \rho)},
$$

with $a, c \in \mathbb{R}$ and $|a| \leq 1 / j$ for $j \neq 0$. (For $j=0$ the constant a does not appear).

Proof. The "only if" part: By Theorem 2.17 there are self-adjoint matrices $M, N$ such that in the helicity representation

$$
V^{h}(t)[f]=\left[e^{i t(M+|\cdot| N)} f\right]
$$

and

$$
\langle j, r|N| j, s\rangle=0 \quad \forall r \neq s, \quad\langle j, r|M| j, s\rangle=c \delta_{r s}
$$

for some constant $c \in \mathbb{R}$. By Theorem 2.14 there are for each $t \in \mathbb{R}$ entire functions $f_{t}^{(j, j, l)}: \mathbb{C} \rightarrow \mathbb{C}$ such that

$$
\left\langle j, r\left|e^{i t(M+z N)}\right| j, s\right\rangle=\delta_{r s} \sum_{l=0}^{2 j}(-1)^{j-s} \sqrt{2 l+1}\left(\begin{array}{ccc}
j & j & l \\
-s & s & 0
\end{array}\right) z^{l} f_{t}^{(j, j, l)}\left(z^{2}\right) .
$$

Let us abbreviate $N_{s}:=\langle j, s|N| j, s\rangle$ and $V_{s}(z, t):=\left\langle j, s\left|e^{i t(M+z N)}\right| j, s\right\rangle=e^{i t\left(c I+z N_{s}\right)}$. If we take the derivative of $V_{s}$ with respect to $z$ at $z=0$, we obtain

$$
i t N_{s} e^{i t c}= \begin{cases}(-1)^{j-s} \sqrt{3}\left(\begin{array}{ccc}
j & j & 1 \\
s & -s & 0
\end{array}\right) f_{t}^{(j, j, 1)}(0), & j \geq 1 / 2 \\
0, & j=0 .\end{cases}
$$

Since

$$
(-1)^{j-s} \sqrt{3}\left(\begin{array}{ccc}
j & j & 1 \\
s & -s & 0
\end{array}\right)=(-1)^{2 j-1} \sqrt{3} \frac{s}{\sqrt{(2 j+1)(j+1) j}},
$$

we have $N_{s}=s a$ for some $a \in \mathbb{R}$. It is

$$
\left\|e^{i t(M+z N)}\right\|=\max _{s \in[j]}\left|e^{i t(c+z s a)}\right|=\max _{s \in[j]}\left|e^{i t z s a}\right|=\max _{s \in[j]} e^{-t s a \operatorname{Im} z}=e^{j|t a||\operatorname{Im} z|} .
$$


Thus to satisfy condition (b) of Theorem 2.14 we must have $|a| \leq 1 / j$ if $j \neq 0$. For $j=0$, the helicity index $s$ is always zero and the constant $a$ does not appear.

The "if" part: Conditions (a) and (b) of Theorem 2.14 are easily verified. It remains to proof condition (c) of the same Theorem. To this end we invert Eq. (7.1).

$$
\begin{aligned}
\rho^{l} f_{t}^{(j, j, l)}\left(\rho^{2}\right) & =\sqrt{2 l+1} \sum_{s \in[j]} V_{s}(\rho, t)(-1)^{j-s}\left(\begin{array}{ccc}
j & j & l \\
-s & s & 0
\end{array}\right) \\
& =\sqrt{2 l+1} e^{i t c} \sum_{s \in[j]} e^{i t a s \rho}(-1)^{j-s}\left(\begin{array}{ccc}
j & j & l \\
-s & s & 0
\end{array}\right) \\
& =\sqrt{2 l+1} e^{i t c} \sum_{n=0}^{\infty} \frac{(i t a)^{n}}{n !} \rho^{n} \sum_{s \in[j]} s^{n}(-1)^{j-s}\left(\begin{array}{ccc}
j & j & l \\
-s & s & 0
\end{array}\right) .
\end{aligned}
$$

By Lemma E.4 and the orthogonality relations for the Wigner $3 j$ symbols we have, for $n<l$,

Hence

$$
\sum_{s \in[j]} s^{n}(-1)^{j-s}\left(\begin{array}{ccc}
j & j & l \\
-s & s & 0
\end{array}\right)=0
$$

$$
f_{t}^{(j, j, l)}\left(\rho^{2}\right)=\sqrt{2 l+1} e^{i t c} \sum_{n=l}^{\infty} \frac{(i t a)^{n}}{n !} \rho^{n-l} \sum_{s \in[j]} s^{n}(-1)^{j-s}\left(\begin{array}{ccc}
j & j & l \\
-s & s & 0
\end{array}\right) .
$$

Using the symmetries of the Wigner $3 j$ symbol we see that

$$
\begin{aligned}
\sum_{s \in[j]} s^{n}(-1)^{j-s}\left(\begin{array}{ccc}
j & j & l \\
-s & s & 0
\end{array}\right) & =\sum_{s \in[j]}(-s)^{n}(-1)^{j+s}\left(\begin{array}{lll}
j & j & l \\
s & -s & 0
\end{array}\right) \\
& =\sum_{s \in[j]}(-s)^{n}(-1)^{j+s}(-1)^{2 j+l}\left(\begin{array}{ccc}
j & j & l \\
-s & s & 0
\end{array}\right) \\
& =\sum_{s \in[j]}(-1)^{l-n} s^{n}(-1)^{j-s}\left(\begin{array}{ccc}
j & j & l \\
-s & s & 0
\end{array}\right) .
\end{aligned}
$$

Thus if $l-n$ is odd, then this expression vanishes. Hence the right hand side of 7.2 is also a function of $\rho^{2}$. Whence $f_{t}^{(j, j, l)}$ has an entire extension.

7.2 Remark. The condition $j|a| \leq 1$ in Theorem 7.1 arises from the causality condition, i.e. the fact that the maximum speed of propagation equals 1.

7.3 Discussion. Let $V$ be the time evolution in Theorem 7.1. With the following Lemma we can determine the energy operator for $V$ in the momentum space representation. It is the matrix multiplication operator corresponding to the function $h: \mathbb{R}^{3} \rightarrow \mathbb{C}^{2 j+1}$,

$$
\begin{aligned}
h(\mathbf{p})_{r s}= & \delta_{r s} c+a\left(r p_{3} \delta_{r s}+\frac{1}{2}((j+r)(j-r+1))^{1 / 2}\left(p_{1}-i p_{2}\right) \delta_{r, s+1}\right. \\
& \left.+\frac{1}{2}((j+r+1)(j-r))^{1 / 2}\left(p_{1}+i p_{2}\right) \delta_{r, s-1}\right) .
\end{aligned}
$$


where $a, c \in \mathbb{R}$ and $|a| \leq 1 / j$ for $j \neq 0$.

For $j=1 / 2$ we have

$$
h(\mathbf{p})=c 1_{2}+\frac{1}{2} a \sigma(\mathbf{p}) .
$$

Hence for $c=0$ and $a= \pm 2$ we obtain the irreducible parts of the Dirac operator with mass zero. Note that the Dirac operator with mass zero corresponds to

$$
h_{0}^{D}(\mathbf{p})=\left(\begin{array}{cc}
0 & \sigma(\mathbf{p}) \\
\sigma(\mathbf{p}) & 0
\end{array}\right) .
$$

The unitary transform corresponding to the matrix

$$
Y:=\frac{1}{\sqrt{2}}\left(\begin{array}{cc}
1_{2} & 1_{2} \\
1_{2} & -1_{2}
\end{array}\right)
$$

commutes with $U_{D^{(1 / 2)}} \oplus U_{D^{(1 / 2)}}$ and satisfies

$$
Y h(\mathbf{p}) Y^{-1}=\left(\begin{array}{cc}
\sigma(\mathbf{p}) & 0 \\
0 & -\sigma(\mathbf{p})
\end{array}\right) .
$$

7.4 Lemma. Let $j \in \mathbb{N} / 2$ and let $M_{r s}=r \delta_{r s}$ for $r, s \in[j]$. Then

$$
\begin{aligned}
\left(D^{(j)}(B(\mathbf{p}))|\mathbf{p}| M D^{(j)}\left(B(\mathbf{p})^{-1}\right)\right)_{r s}= & r p_{3} \delta_{r s}+\frac{1}{2}((j+r)(j-r+1))^{1 / 2}\left(p_{1}-i p_{2}\right) \delta_{r, s+1} \\
& +\frac{1}{2}((j+r+1)(j-r))^{1 / 2}\left(p_{1}+i p_{2}\right) \delta_{r, s-1} .
\end{aligned}
$$

Proof. We have

$$
D^{(j)}\left(\left(\begin{array}{cc}
a & 0 \\
0 & b
\end{array}\right)\right)_{r s}=a^{j+r} b^{j-r} \delta_{r s}
$$

for every $a, b \in \mathbb{C}$. Hence

$$
M=\left.\partial_{\alpha} D^{(j)}\left(\left(\begin{array}{cc}
\alpha & 0 \\
0 & 1
\end{array}\right)\right)\right|_{\alpha=1}-j I
$$

whence

$$
D^{(j)}(B(\mathbf{p})) M D^{(j)}\left(B(\mathbf{p})^{-1}\right)=\left.\partial_{\alpha} D^{(j)}\left(B(\mathbf{p})\left(\begin{array}{ll}
\alpha & 0 \\
0 & 1
\end{array}\right) B(\mathbf{p})^{-1}\right)\right|_{\alpha=1}-j I .
$$

Using

$$
\left.\partial_{\alpha}\left(B(\mathbf{p})\left(\begin{array}{cc}
\alpha & 0 \\
0 & 1
\end{array}\right) B(\mathbf{p})^{-1}\right)\right|_{\alpha=1}=\frac{1}{2|\mathbf{p}|}\left(\begin{array}{cc}
|\mathbf{p}|+p_{3} & p_{1}-i p_{2} \\
p_{1}+i p_{2} & |\mathbf{p}|-p_{3}
\end{array}\right)
$$

and

$$
\begin{aligned}
\left(d D^{(j)}(I) \circ M\right)_{r s}= & (j+r) M_{11} \delta_{r s}+((j+r)(j-r+1))^{1 / 2} M_{12} \delta_{r, s+1} \\
& +((j+r+1)(j-r))^{1 / 2} M_{21} \delta_{r, s-1}+(j-m) M_{22} \delta_{r s} \quad \forall M \in \mathbb{C}^{2 \times 2}
\end{aligned}
$$

completes the proof. 


\section{$8 \quad$ Asymptotically Localized States and Hegerfeldt's Theorem}

The occurrence of negative energies in causal localizations (see 2.4) will be discussed in more detail. After reviewing Hegerfeldt's Theorem, which says that positive energy states cannot be localized, we show that there is still a meaningful notion of localization for such states.

8.1 Theorem (Hegerfeldt Heg74). Let (W,E) be a relativistic causal localization. Then there are no non-zero positive energy states which are localized in a bounded set $\Delta \in \mathscr{B}\left(\mathbb{R}^{3}\right)$, i.e.

$$
M:=\left\{\psi \in \mathscr{H}: P_{+} \psi=\psi, E(\Delta) \psi=\psi, \text { for some bounded } \Delta \in \mathscr{B}\left(\mathbb{R}^{3}\right)\right\}=\{0\},
$$

where $P_{+}:=\frac{1}{2}(I+\operatorname{sgn}(H))$. The same statement holds for negative energy states.

Proof. (Adapted from [Heg74]). By Discussion 2.5 we may assume that $E$ is complete, so that $E\left(\Delta_{t}\right)$ is well-defined for every $\Delta \subset \mathbb{R}^{3}$ and $t>0$.

Suppose there exists a $\psi \in M$ such that $\|\psi\|=1$. Let $t>0$ and $\mathbf{a} \in \mathbb{R}^{3}$. By means of covariance and causality, $U(\mathbf{a}) V(t) \psi$ must be localized in $\left(\Delta_{t}+\mathbf{a}\right)$. Indeed, using Corollary 2.10 we find

$$
\begin{aligned}
U(\mathbf{a}) V(t) \psi & =U(\mathbf{a}) V(t) E(\Delta) \psi=U(\mathbf{a}) E\left(\Delta_{t}\right) V(t) E(\Delta) \psi \\
& =E\left(\Delta_{t}+\mathbf{a}\right) U(\mathbf{a}) V(t) \psi
\end{aligned}
$$

Because $\Delta$ is bounded, there exists an $R_{t}>0$ such that $\Delta \cap\left(\Delta_{t}+\mathbf{a}\right)=\varnothing$ for all $|\mathbf{a}|>R_{t}$. This implies that

$$
E(\Delta) U(\mathbf{a}) V(t) \psi=0 \quad \forall|\mathbf{a}|>R_{t} .
$$

The mass square operator $C$ commutes with $V, U$ and $E$, thus if $P_{\mu}$ are the projections onto the eigenspaces of $C$ and $\psi_{\mu}:=P_{\mu} \psi$, then

$$
E(\Delta) U(\mathbf{a}) V(t) \psi_{\mu}=0 \quad \forall|\mathbf{a}|>R_{t} .
$$

Taking the scalar product with $\psi_{\mu}$ and using $E(\Delta) \psi_{\mu}=\psi_{\mu}$ we find

$$
\left\langle\psi_{\mu}, U(\mathbf{a}) V(t) \psi_{\mu}\right\rangle=0 \quad \forall|\mathbf{a}|>R_{t} .
$$

Since this expression is basis independent, we may use the representation in which $H$ is diagonal. Then Eq. (8.1) becomes

$$
\int_{\mathbb{R}^{3}} \sum_{\sigma}\left|\psi_{\sigma, \mu}(\mathbf{p})\right|^{2} e^{-i \mathbf{p} \cdot \mathbf{a}} e^{i t \sqrt{\mu^{2}+\mathbf{p}^{2}}} \mathrm{~d} \mathbf{p}=0 \quad \forall|\mathbf{a}|>R_{t},
$$

where $\sigma$ denotes multiplicity and helicity indices.

By the Paley-Wiener Theorem $f_{t}(\mathbf{p}):=\sum_{\sigma}\left|\psi_{\sigma, \mu}(\mathbf{p})\right|^{2} e^{i t \sqrt{\mu^{2}+\mathbf{p}^{2}}}$ has an entire extension on $\mathbb{C}^{3}$. But because of the square root this is impossible, which can be seen by standard methods. 
8.2 Discussion. Let $\Delta$ be a bounded set in $\mathscr{B}\left(\mathbb{R}^{3}\right)$. By Hegerfeldt's Theorem we know that if $0 \neq \psi \in \mathscr{H}$ is a positive energy state, then it cannot be localized in $\Delta$. It is therefore natural to ask if there are positive energy states that can be localized arbitrarily good, i.e. given $\varepsilon>0$ is it possible to find a normalized $\psi \in \mathscr{H}$ such that $P_{+} \psi=\psi$ and $\left\|E\left(\Delta^{c}\right) \psi\right\|<\varepsilon$.

To answer this question we need some basic facts about the dilation operator.

8.3. Definition and Discussion. Let $\mathscr{H}:=L^{2}\left(\mathbb{R}^{3}, \mathbb{C}^{m}\right)$. For $\lambda>0$ the dilation operator is defined as $D_{\lambda}: \mathscr{H} \rightarrow \mathscr{H}$,

$$
D_{\lambda}[g]:=\lambda^{-3 / 2}[g(\cdot / \lambda)] \quad \forall[g] \in \mathscr{H} .
$$

We have the following properties:

(i) $\lambda \mapsto D_{\lambda}$ is a unitary group homomorphism for $\left(\mathbb{R}_{>0}, \cdot\right)$ in $L(\mathscr{H})$.

(ii) The Fourier transform of $D_{\lambda}$ satisfies $\hat{D}_{\lambda}:=\mathscr{F} D_{\lambda} \mathscr{F}^{-1}=D_{1 / \lambda}$ for all $\lambda>0$.

(iii) In the helicity representation $D_{\lambda}$ is given by $D_{\lambda}^{h}=D_{1 / \lambda}$.

(iv) Let $E: \mathscr{B}\left(\mathbb{R}^{3}\right) \rightarrow L(\mathscr{H})$ be the canonical projection-valued measure. Then we have the covariance

$$
D_{\lambda} E(\Delta) D_{\lambda}^{-1}=E(\lambda \Delta) \quad \forall \lambda>0, \Delta \in \mathscr{B}\left(\mathbb{R}^{3}\right)
$$

(v) If $S \in L(\mathscr{H})$ is a matrix multiplication operator, i.e. $S[g]=[h g]$, for some measurable bounded matrix-valued function $h: \mathbb{R}^{3} \rightarrow \mathbb{C}^{m \times m}$, then

$$
D_{\lambda} S D_{\lambda}^{-1}[g]=[h(\cdot / \lambda) g] \quad \forall[g] \in \mathscr{H} .
$$

Note that if $\hat{T}$ is a matrix multiplication operator in the momentum space representation, i.e. $\hat{T}[f]=[k f]$ for some measurable bounded matrix-valued function $k: \mathbb{R}^{3} \rightarrow \mathbb{C}^{m \times m}$, then

$$
\hat{D}_{\lambda} \hat{T} \hat{D}_{\lambda}^{-1}[f]=D_{1 / \lambda} \hat{T} D_{1 / \lambda}^{-1}[f]=[k(\lambda \cdot) f] \quad \forall[f] \in \mathscr{H} .
$$

Proof. (i) It is easy to see that $D_{1}=I, D_{\alpha} D_{\beta}=D_{\alpha \beta}$ and $D_{\alpha}^{*}=D_{1 / \alpha}$ for all $\alpha, \beta>0$. Thus $D_{\alpha}^{*} D_{\alpha}=D_{\alpha} D_{\alpha}^{*}=D_{1}=I$.

(ii) For $f \in L^{1} \cap L^{2}, \lambda>0$ we have $D_{\lambda} \mathscr{F}^{-1}[f]=\mathscr{F}^{-1} D_{1 / \lambda}[f]$, where $\mathscr{F}$ denotes the Fourier transform. Since $L^{1} \cap L^{2}$ is dense in $L^{2}$, we have $\mathscr{F} D_{\lambda} \mathscr{F}^{-1}=D_{1 / \lambda}$.

(iii) This follows from (ii) and the fact that $B(\lambda \mathbf{p})=B(\mathbf{p})$ for all $\lambda>0, \mathbf{p} \in \mathbb{R}^{3}$, see Eq. (1.2) fol.

(iv) and (v) are obvious. 
8.4 Lemma. Let $(V, U, E)$ be the standard Dirac system and let $P_{+}:=\frac{1}{2}(I+\operatorname{sgn}(H))$ be the projection onto the positive energy states, i.e. in the momentum space representation

$$
\hat{P}_{+}[f]=[k f], \quad k(\mathbf{p}):=\frac{1}{2 \epsilon(\mathbf{p})}\left(\begin{array}{cc}
(\epsilon(\mathbf{p})+m) 1_{2} & \sigma(\mathbf{p}) \\
\sigma(\mathbf{p}) & (\epsilon(\mathbf{p})-m) 1_{2}
\end{array}\right),
$$

for $f \in \mathscr{H}:=L^{2}\left(\mathbb{R}^{3}, \mathbb{C}^{4}\right)$. Let $D_{\lambda}$ be the dilation operator. Then for every $f \in \mathscr{H}$,

$$
\hat{D}_{n} \hat{P}_{+} \hat{D}_{n}^{-1}[f] \stackrel{n \rightarrow \infty}{\longrightarrow} \hat{Q}_{+}[f]
$$

where $\hat{Q}_{+} \in L(\mathscr{H})$ is the self-adjoint projection given by $\hat{Q}_{+}[f]:=[q f]$,

$$
q(\mathbf{p}):=\frac{1}{2}\left(\begin{array}{cc}
1_{2} & \sigma(\mathbf{p}) /|\mathbf{p}| \\
\sigma(\mathbf{p}) /|\mathbf{p}| & 1_{2}
\end{array}\right) \quad \forall \mathbf{p} \in \mathbb{R}^{3} \backslash\{0\}, \quad q(0):=1_{4} .
$$

However, it is $\left\|\hat{D}_{\lambda} \hat{P}_{+} \hat{D}_{\lambda}^{-1}-\hat{Q}_{+}\right\|^{2} \geq 1 / 8$ for all $\lambda>0$.

Proof. It is clear that $q(\mathbf{p})$ is a self-adjoint projection for each $\mathbf{p} \in \mathbb{R}^{3}$ and that each component of $q$ is a bounded measurable function. Thus $\hat{Q}_{+}$is a self-adjoint projection. Let $f \in \mathscr{H}$. By $8.3(\mathrm{v})$ we have $\hat{D}_{n} \hat{P}_{+} \hat{D}_{n}^{-1}[f]=[k(n \cdot) f]$. Clearly, $k(n \mathbf{p}) \stackrel{n \rightarrow \infty}{\longrightarrow} q(\mathbf{p})$ for all $\mathbf{p} \in \mathbb{R}^{3}$. Since $k(n \mathbf{p})$ is a self-adjoint projection for all $n \in \mathbb{N}$ and $\mathbf{p} \in \mathbb{R}^{3}$, we have

$$
g_{n}(\mathbf{p}):=\|(k(n \mathbf{p})-q(\mathbf{p})) f(\mathbf{p})\|^{2} \leq 4\|f(\mathbf{p})\|^{2} \quad \forall n \in \mathbb{N}, \mathbf{p} \in \mathbb{R}^{3} .
$$

Hence, by Lebesgue's Dominated Convergence Theorem

$$
\lim _{n \rightarrow \infty}\left\|\left(\hat{D}_{n} \hat{P}_{+} \hat{D}_{n}^{-1}-\hat{Q}_{+}\right) f\right\|^{2}=\lim _{n \rightarrow \infty} \int_{\mathbb{R}^{3}} g_{n}(\mathbf{p}) d \mathbf{p}=\int_{\mathbb{R}^{3}} \lim _{n \rightarrow \infty} g_{n}(\mathbf{p}) d \mathbf{p}=0 .
$$

Let $\lambda>0$ and consider the function $f_{\lambda}: \mathbb{R}^{3} \backslash\{0\} \rightarrow \mathbb{R}^{4}$,

$$
f_{\lambda}(\mathbf{p}):=\left(\frac{m \lambda}{2 \pi^{2}}\right)^{1 / 2} \frac{1}{|\mathbf{p}| \sqrt{\lambda^{2} \mathbf{p}^{2}+m^{2}}} e_{1}
$$

Then $\left\|f_{\lambda}\right\|=1$ and

$$
\begin{aligned}
\left\|\hat{D}_{\lambda} \hat{P}_{+} \hat{D}_{\lambda}^{-1}-\hat{Q}_{+}\right\|^{2} & \geq\left\|\left(\hat{D}_{\lambda} \hat{P}_{+} \hat{D}_{\lambda}^{-1}-\hat{Q}_{+}\right) f_{\lambda}\right\|^{2} \\
& =\frac{m \lambda}{8 \pi^{2}} \int_{\mathbb{R}^{3}} \frac{m^{2}+(\lambda|\mathbf{p}|-\epsilon(\lambda \mathbf{p}))^{2}}{|\mathbf{p}|^{2} \epsilon(\lambda \mathbf{p})^{4}} d \mathbf{p} \\
& \geq \frac{m \lambda}{2 \pi} \int_{0}^{\infty}\left(\frac{m}{\lambda^{2} \rho^{2}+m^{2}}\right)^{2} d \rho=\frac{1}{8},
\end{aligned}
$$

where we used $\epsilon(\lambda \mathbf{p})^{2}-\lambda|\mathbf{p}| \epsilon(\lambda \mathbf{p}) \geq m^{2} / 2$. This completes the proof.

8.5 Note. Let $(V, U, E)$ be a relativistic extendable causal localization. From Theorem 5.8 and 5.10 it follows that $(H, E)$ is unitarily equivalent to $\left(H^{D} \otimes I, E^{D} \otimes I\right)$, where $H^{D}$ is the Dirac operator and $E^{D}$ is the canonical projection-valued measure. So by means of this equivalence it is possible to define a dilation operator $D_{\lambda}$ for every relativistic extendable causal localization. Moreover, $D_{\lambda}$ will satisfy all previous statements. 
8.6 Definition. Let $(V, U, E)$ be a causal localization on a complex separable Hilbert space $\mathscr{H}$. Let $K_{r}:=\left\{\mathbf{x} \in \mathbb{R}^{3}:|\mathbf{x}| \leq r\right\}$ for $r>0$. A sequence $\left(\psi_{n}\right)_{n \in \mathbb{N}}$ in $\mathscr{H}$ is said to be an asymptotically localized state of positive energy if each $\psi_{n}$ is a normalized state of positive energy, i.e.

$$
\left\|\psi_{n}\right\|=1, \quad P_{+} \psi_{n}=\psi_{n} \quad \forall n \in \mathbb{N},
$$

and if

$$
\left\|E\left(K_{1}\right) \psi_{n}\right\| \stackrel{n \rightarrow \infty}{\longrightarrow} 1 .
$$

Note that the last condition is equivalent to

$$
\left\|E\left(K_{1}\right) \psi_{n}-\psi_{n}\right\| \stackrel{n \rightarrow \infty}{\longrightarrow} 0 .
$$

One can also consider a set $\Delta \in \mathscr{B}\left(\mathbb{R}^{3}\right)$ other than $K_{1}$ and say $\left(\psi_{n}\right)_{n \in \mathbb{N}}$ is an asymptotically localized state of positive energy for $\Delta$ if each $\psi_{n}$ is a normalized state of positive energy and if

$$
\left\|E(\Delta) \psi_{n}\right\| \stackrel{n \rightarrow \infty}{\longrightarrow} 1 .
$$

However, if $\left(\psi_{n}\right)_{n}$ is an asymptotically localized state of positive energy and if $\mathbf{a} \in \mathbb{R}^{3}$, then, since by covariance $E\left(K_{r}+\mathbf{a}\right)=U(\mathbf{a}, I) D_{r} E\left(K_{1}\right) D_{r}^{-1} U(\mathbf{a}, I)^{-1}$, we have

$$
\left\|E\left(K_{r}+\mathbf{a}\right) \psi_{n}^{\prime}\right\| \stackrel{n \rightarrow \infty}{\longrightarrow} 1 \text {, }
$$

where $\psi_{n}^{\prime}:=U(\mathbf{a}, I) D_{r} \psi_{n}$. That is to say that $\left(\psi_{n}^{\prime}\right)_{n \in \mathbb{N}}$ is an asymptotically localized state of positive energy for $K_{r}+\mathbf{a}$.

The following Lemma shows that asymptotically localized state of positive energy obey the causality condition.

8.7 Lemma. Let $(V, U, E)$ be a causal localization on a complex separable Hilbert space $\mathscr{H}$ and let $\left(\psi_{n}\right)_{n \in \mathbb{N}}$ be an asymptotically localized state of positive energy for some $\Delta \in \mathscr{B}\left(\mathbb{R}^{3}\right)$. Then for every $t \in \mathbb{R},\left(V(t) \psi_{n}\right)_{n \in \mathbb{N}}$ is an asymptotically localized state of positive energy for $\Delta_{t}$.

Proof. By the unitarity of $V(t)$ it is $\left\|V(t) \psi_{n}\right\|=1$ for all $n \in \mathbb{N}$, and since $P_{+}$commutes with $V(t),\left(V(t) \psi_{n}\right)_{n \in \mathbb{N}}$ is a sequence of positive energy states. Using Corollary 2.10 we find

$$
\begin{aligned}
& \left\|E_{c}\left(\Delta_{t}\right) V(t) \psi_{n}-V(t) \psi_{n}\right\| \\
& \quad=\left\|E_{c}\left(\Delta_{t}\right) V(t)\left(\psi_{n}-E(\Delta) \psi_{n}\right)+E_{c}\left(\Delta_{t}\right) V(t) E(\Delta) \psi_{n}-V(t) \psi_{n}\right\| \\
& \quad \leq\left\|E_{c}\left(\Delta_{t}\right) V(t)\left(\psi_{n}-E(\Delta) \psi_{n}\right)\right\|+\left\|E_{c}\left(\Delta_{t}\right) V(t) E(\Delta) \psi_{n}-V(t) \psi_{n}\right\| \\
& \quad \leq\left\|\psi_{n}-E(\Delta) \psi_{n}\right\|+\left\|V(t) E(\Delta) \psi_{n}-V(t) \psi_{n}\right\| \\
& \quad=2\left\|E(\Delta) \psi_{n}-\psi_{n}\right\| \stackrel{n \rightarrow \infty}{\longrightarrow} 0 .
\end{aligned}
$$

8.8 Theorem. Let $(V, U, E)$ be a relativistic extendable causal localization on a complex separable Hilbert space $\mathscr{H}$. Let $\psi \in \mathscr{H}$ such that $Q_{+} \psi \neq 0$. Then there exists a $k \in \mathbb{N}$ such that $\left\|P_{+} D_{n}^{-1} \psi\right\| \neq 0$ for all $n \geq k$ and

$$
\left(\left\|P_{+} D_{n}^{-1} \psi\right\|^{-1} P_{+} D_{n}^{-1} \psi\right)_{n \geq k}
$$

is an asymptotically localized state of positive energy. 
Proof. By Note 8.5 it suffices to consider the Dirac case. We have

$$
\left\|P_{+} D_{n}^{-1} \psi\right\|=\left\|D_{n} P_{+} D_{n}^{-1} \psi\right\| \stackrel{n \rightarrow \infty}{\longrightarrow}\left\|Q_{+} \psi\right\| \neq 0
$$

thus $\left\|P_{+} D_{n}^{-1} \psi\right\| \neq 0$ for $n$ large enough. Moreover,

$$
\left\|P_{+} D_{n}^{-1} \psi\right\|^{-1} \stackrel{n \rightarrow \infty}{\longrightarrow}\left\|Q_{+} \psi\right\|^{-1} .
$$

Then

$$
\begin{aligned}
\left\|E\left(K_{1}\right) P_{+} D_{n}^{-1} \psi\right\| & =\left\|D_{n} E\left(K_{1}\right) D_{n}^{-1} D_{n} P_{+} D_{n}^{-1} \psi\right\| \\
& =\left\|E\left(K_{n}\right) D_{n} P_{+} D_{n}^{-1} \psi\right\| \stackrel{n \rightarrow \infty}{\longrightarrow}\left\|Q_{+} \psi\right\|,
\end{aligned}
$$

since

$$
\begin{aligned}
\left\|E\left(K_{n}\right) D_{n} P_{+} D_{n}^{-1} \psi-Q_{+} \psi\right\| \leq & \left\|E\left(K_{n}\right) D_{n} P_{+} D_{n}^{-1} \psi-E\left(K_{n}\right) Q_{+} \psi\right\| \\
& +\left\|E\left(K_{n}\right) Q_{+} \psi-Q_{+} \psi\right\| \\
\leq & \left\|D_{n} P_{+} D_{n}^{-1} \psi-Q_{+} \psi\right\|+\left\|E\left(\mathbb{R}^{3} \backslash K_{n}\right) Q_{+} \psi\right\| \stackrel{n \rightarrow \infty}{\longrightarrow} 0 .
\end{aligned}
$$

This completes the proof.

8.9 Discussion. We can change the perspective from positive energy states to localized states by interchanging $P_{+}$and $E(\Delta)$.

Let $(V, U, E)$ be a relativistic extendable causal localization on a complex separable Hilbert space $\mathscr{H}$ and let $\psi_{n} \in \mathscr{H}$ for each $n \in \mathbb{N}$. Then $\left(\psi_{n}\right)_{n \in \mathbb{N}}$ is said to be a localized state of asymptotically positive energy if for all $n \in \mathbb{N},\left\|\psi_{n}\right\|=1$, $E\left(K_{1}\right) \psi_{n}=\psi_{n}$ and

$$
\left\|P_{+} \psi_{n}\right\| \stackrel{n \rightarrow \infty}{\longrightarrow} 1 \text {. }
$$

Note that the last condition is equivalent to

$$
\left\|P_{+} \psi_{n}-\psi_{n}\right\| \stackrel{n \rightarrow \infty}{\longrightarrow} 0 .
$$

The analogous version of Lemma 8.7 is obvious. The corresponding version of Theorem 8.8 is stated in the next Theorem.

8.10 Theorem. Let $\phi \in \mathscr{H}$ such that $Q_{+} \phi \neq 0$. Put $\psi:=Q_{+} \phi$. Then there exists a $k \in \mathbb{N}$ such that $\left\|E\left(K_{1}\right) D_{n}^{-1} \psi\right\| \neq 0$ for all $n \geq k$ and

$$
\left(\left\|E\left(K_{1}\right) D_{n}^{-1} \psi\right\|^{-1} E\left(K_{1}\right) D_{n}^{-1} \psi\right)_{n \geq k}
$$

is a localized state of asymptotically positive energy.

Proof. Again, we may assume that $H$ is the Dirac operator. By Lemma 8.4 it is

$$
\left\|E\left(K_{1}\right) D_{n}^{-1} \psi\right\|=\left\|D_{n} E\left(K_{1}\right) D_{n}^{-1} \psi\right\|=\left\|E\left(K_{n}\right) \psi\right\| \stackrel{n \rightarrow \infty}{\longrightarrow}\|\psi\| \neq 0,
$$


thus $\left\|E\left(K_{1}\right) D_{n}^{-1} \psi\right\| \neq 0$ for $n$ large enough. Moreover,

$$
\left\|E\left(K_{1}\right) D_{n}^{-1} \psi\right\|^{-1} \stackrel{n \rightarrow \infty}{\longrightarrow}\|\psi\|^{-1} .
$$

Then

$$
\begin{aligned}
\left\|P_{+} E\left(K_{1}\right) D_{n}^{-1} \psi\right\| & =\left\|D_{n} P_{+} D_{n}^{-1} D_{n} E\left(K_{1}\right) D_{n}^{-1} \psi\right\| \\
& =\left\|D_{n} P_{+} D_{n}^{-1} E\left(K_{n}\right) \psi\right\| \stackrel{n \rightarrow \infty}{\longrightarrow}\left\|Q_{+} \psi\right\|=\|\psi\|,
\end{aligned}
$$

since

$$
\begin{aligned}
\left\|D_{n} P_{+} D_{n}^{-1} E\left(K_{n}\right) \psi-Q_{+} \psi\right\| & \leq\left\|D_{n} P_{+} D_{n}^{-1}\left(E\left(K_{n}\right) \psi-\psi\right)+\left(D_{n} P_{+} D_{n}^{-1}-Q_{+}\right) \psi\right\| \\
& \leq\left\|E\left(K_{n}\right) \psi-\psi\right\|+\left\|\left(D_{n} P_{+} D_{n}^{-1}-Q_{+}\right) \psi\right\| \stackrel{n \rightarrow \infty}{\longrightarrow} 0 .
\end{aligned}
$$

This completes the proof. 


\section{Open Problems}

At this point we like to address some open problems.

9.1. Let $(V, U, F)$ be a causal unsharp localization on a complex separable Hilbert space $\mathscr{H}$. Then $(V, U, F)$ is said to have a sharp extension if there exists a causal localization $\left(V^{\prime}, U^{\prime}, E\right)$ on a complex separable Hilbert space $\mathscr{H}^{\prime}$ such that $\mathscr{H} \subset \mathscr{H}^{\prime}$, $\left.V^{\prime}\right|_{\mathscr{H}}=V,\left.U^{\prime}\right|_{\mathscr{H}}=U$ and $F(\Delta)=i n^{*} E(\Delta)$ in for every $\Delta \in \mathscr{B}\left(\mathbb{R}^{3}\right)$, where in $: \mathscr{H} \hookrightarrow$ $\mathscr{H}^{\prime}$ is the inclusion map. The hypothesis then is: every relativistic extendable causal unsharp localization has a sharp extension.

In this context Naimark's Dilation Theorem (see the Appendix of [RSN82]) and [[Scu77] (15) and (16)] might be useful.

9.2. If $(W, U, E)$ is an irreducible relativistic causal localization, then $(V, U, E)$, where $V$ is the time evolution part of $W$, is in general not irreducible. We know all irreducible relativistic extendable causal localizations, but we do not know in what ways these can be combined to result in irreducible relativistic causal localizations. So the problem is to characterize all relativistic causal localizations.

9.3. Since we only considered finite localization, it might be interesting to study infinite localizations, i.e. countable direct sums of finite localizations. The main problem here is to adapt 12.6 to infinite dimensions.

9.4. In Note 6.2 we concluded that there are different extensions for the Dirac system. Still there is no real satisfactory answer weather these extensions are physically equivalent or not. However, we have a convenient formula (see Eq. (6.4)) for the boosts of the Dirac system and the Dirac tensor system.

9.5. Characterize all finite causal localizations. As seen in section 7 there are nonrelativistic causal localizations. These may describe causal propagation in a solid-state. We have seen that the energy operator for a finite causal localization corresponds in the helicity representation to a linear matrix-valued function $\mathbb{R}_{\geq 0} \ni \rho \mapsto M+\rho N$, where $M$ and $N$ are self-adjoint matrices (cf. Theorem 2.17). The relativistic condition then implies $M N+N M=0, N^{2}=I$ and $M^{2}>0$. Without this condition it will be more difficult, but certainly not impossible, to study the consequences of part (c) of Theorem 2.14 .

9.6. In Theorem 8.8 the existence of asymptotically localized state of positive energy was proven only for closed balls (in fact only for the closed unit ball, but by means of the covariance the existence applies to every closed ball). So it might be interesting to study the general case. 


\section{Part II}

\section{Paley-Wiener Theorems}

10.1. Motivation. Consider the abstract the causality condition for a bounded operator $V$ in $\mathscr{H}:=L^{2}\left(\mathbb{R}^{d}, \mathbb{C}^{m}\right)$ :

$$
V E(\Delta)=E\left(\Delta^{\prime}\right) V E(\Delta)
$$

for some open balls $\Delta \subset \Delta^{\prime}$ centered at the origin, where $E$ is the canonical projectionvalued measure (cf. Lemma 2.11). Suppose $V$ satisfies

$$
V[g]:=\left[\mathscr{F}^{-1} A \mathscr{F} g\right] \quad \forall[g] \in \mathscr{H},
$$

for some measurable bounded matrix-valued function $A: \mathbb{R}^{d} \rightarrow \mathbb{C}^{m \times m}$, i.e. the Fourier transform of $V$ is a matrix multiplication operator. Note that every time evolution is of this from. To show what we are aiming for assume $m=1$ and suppose $V$ satisfies the abstract causality condition. For some $q \in L^{2}$ with support in $\Delta$ the classical PaleyWiener Theorem (see below) implies that $\mathscr{F} q$ has an entire exponentially bounded extension. From Eq. (10.1) it follows that $\mathscr{F}^{-1} A \mathscr{F} q$ is supported in $\Delta^{\prime}$. Thus $A \mathscr{F} q$ also has an entire exponentially bounded extension. The obvious questions then are: does $A$ have an entire extension and, if so, is it exponentially bounded? As we will see in this section, both questions can be answered affirmatively.

10.2. Notation. Let $d \in \mathbb{N}$. We use the following definitions for the scalar product and the norm in $\mathbb{C}^{d}$ :

$$
\langle z, w\rangle:=\sum_{k=1}^{d} \overline{z_{k}} w_{k}, \quad|z|:=\langle z, z\rangle^{1 / 2} \quad \text { for } z, w \in \mathbb{C}^{d}
$$

where $\overline{z_{k}}$ denotes the complex conjugate of $z_{k}$. The scalar product $\langle\cdot, \cdot\rangle$ we are using is linear in the second variable - this is the convention used in quantum mechanics. The space $\mathbb{R}^{d}$ will be considered as a subspace of $\mathbb{C}^{d}$, i.e. $\mathbb{R}^{d}=\left\{z \in \mathbb{C}^{d}: \bar{z}=z\right\}$. Then $|x|$ coincides with the usual Euclidean norm of $x \in \mathbb{R}^{d}$, and $\langle x, y\rangle$ coincides with the usual scalar product for $x, y \in \mathbb{R}^{d}$. By means of this embedding it is clear what we mean by $\langle x, z\rangle,\langle z, x\rangle$ and $|\operatorname{Im} z|$ for $x \in \mathbb{R}^{d}$ and $z \in \mathbb{C}^{d}$.

If $A \in \mathbb{C}^{m \times m}$ is a matrix, $\|A\|$ will denote the operator norm of $A$, i.e.

$$
\|A\|:=\sup _{|z|=1}|A z| .
$$

If $A: \mathbb{R}^{d} \rightarrow \mathbb{C}^{m \times m}$ is a bounded continuous matrix-valued function we will use the following definition

$$
\|A\|_{\infty}:=\sup _{x \in \mathbb{R}^{d}}\|A(x)\|
$$


10.3 Definition. An entire function $f: \mathbb{C}^{d} \rightarrow \mathbb{C}^{m \times m}$ is said to be exponentially bounded if

$$
\|f(z)\| \leq C e^{R|z|} \quad \forall z \in \mathbb{C}^{d},
$$

for some constants $C>0$ and $R>0$. Such functions are also called functions of exponential type.

The following Theorem due to Plancherel and Pólya [PP37] is a generalization of the Paley-Wiener Theorem. The version presented here is adapted from [ Ron74] Chapter $3 \S 4$ p. 171].

10.4. Theorem (Plancherel-Pólya). Let $f: \mathbb{C}^{d} \rightarrow \mathbb{C}$. Then $f$ is an entire function of exponential type and $\left.f\right|_{\mathbb{R}^{d}} \in L^{2}\left(\mathbb{R}^{d}\right)$ if and only if there exists a $\phi \in L^{2}\left(\mathbb{R}^{d}\right)$ which vanishes outside some bounded set such that

$$
f(z)=(2 \pi)^{-d / 2} \int_{\mathbb{R}^{d}} e^{-i\langle p, z\rangle} \phi(p) d p \quad \forall z \in \mathbb{C}^{d} .
$$

Moreover, if (10.2) holds, then the support function $H_{D}$ of $D$, where $D$ is the smallest convex set in $\mathbb{R}^{d}$ such that $\left.\phi\right|_{D^{c}}=0$, coincides with the P-indicator $h_{f}$ of $f$.

Recall that $H_{B}: \mathbb{R}^{d} \rightarrow \mathbb{R}$

$$
H_{B}(\lambda):=\sup _{x \in B}\langle\lambda, x\rangle
$$

and $h_{f}: \mathbb{R}^{d} \rightarrow \mathbb{R}$

$$
h_{f}(\lambda):=\sup _{x \in \mathbb{R}^{d}} h_{f}(\lambda, x),
$$

where

$$
h_{f}(\lambda, x):=\limsup _{r \rightarrow \infty} \frac{\log |f(x+i r \lambda)|}{r} \quad\left(x, \lambda \in \mathbb{R}^{d}\right) .
$$

The "if" part of this Theorem has the following useful form:

10.5 Lemma. Let $\phi \in L^{2}\left(\mathbb{R}^{d}\right)$ vanish almost everywhere outside the compact ball with radius $R>0$ centered at the origin. Then $f: \mathbb{C}^{d} \rightarrow \mathbb{C}$,

$$
f(z):=(2 \pi)^{-d / 2} \int_{\mathbb{R}^{d}} e^{-i\langle p, z\rangle} \phi(p) d p
$$

is entire, $\left.f\right|_{\mathbb{R}^{d}} \in L^{2}\left(\mathbb{R}^{d}\right)$ and there exists a constant $C>0$ such that

$$
|f(z)| \leq C e^{R|\operatorname{Im} z|} \quad \forall z \in \mathbb{C}^{d} .
$$

Proof. Since $p \mapsto \phi(p) e^{\langle p, y\rangle}$ is a function in $L^{1}$ for all $y \in \mathbb{R}^{d}$, we see that

$$
f(x+i y):=(2 \pi)^{-d / 2} \int_{\mathbb{R}^{d}} e^{-i\langle p, x+i y\rangle} \phi(p) d p
$$

is a well-defined function on $\mathbb{C}^{d}$. If $\left(z_{n}\right)_{n \in \mathbb{N}}$ converges to $z$ then there exists an $S>0$ such that $\left|\operatorname{Im} z_{n}\right|<S$ for all $n \in \mathbb{N}$, hence $e^{|\cdot| S} \phi(\cdot)$ is an integrable dominating function 
for $e^{-i\left\langle\cdot, z_{n}\right\rangle} \phi(\cdot)$, whence, by Lebesgue's Dominated Convergence Theorem, $f\left(z_{n}\right) \stackrel{n \rightarrow \infty}{\longrightarrow}$ $f(z)$. Thus $f$ is continuous.

Since $\left[\left.f\right|_{\mathbb{R}^{d}}\right]$ is the Fourier transform of $\phi$, we have $\left.f\right|_{\mathbb{R}^{d}} \in L^{2}$. To show that $f$ is entire it suffices to see that $\psi: \mathbb{C} \rightarrow \mathbb{C}$,

$$
\psi(z):=f\left(a_{1}, \ldots, a_{k-1}, z, a_{k+1}, \ldots, a_{d}\right)
$$

is entire [[Die69] (9.9.4)], where $a_{j} \in \mathbb{C}$. Fix $z \in \mathbb{C}$ and let $\left(\xi_{n}\right)_{n \in \mathbb{N}}$ be a null sequence in $\mathbb{C} \backslash\{0\}$. Then there exists a constant $r>0$ such that

$$
\begin{aligned}
\frac{\left|e^{-i p_{k}\left(z+\xi_{n}\right)}-e^{-i p_{k} z}\right|}{\left|\xi_{n}\right|}|\phi(p)| & \leq\left|e^{-i p_{k} z}\right| \frac{\left|e^{-i p_{k} \xi_{n}}-1\right|}{\left|\xi_{n}\right|}|\phi(p)| \leq\left|e^{-i p_{k} z}\right| \frac{e^{\left|p_{k} \xi_{n}\right|}-1}{\left|\xi_{n}\right|}|\phi(p)| \\
& \leq\left|e^{-i p_{k} z}\right| \frac{e^{\left|R \xi_{n}\right|}-1}{\left|\xi_{n}\right|}|\phi(p)| \leq r\left|e^{-i p_{k} z}\right||\phi(p)|
\end{aligned}
$$

for all $n \in \mathbb{N}$ and for all $p \in \mathbb{R}^{d}$. Thus by Lebesgue's Theorem of Dominated Convergence $\left(\psi\left(z+\xi_{n}\right)-\psi(z)\right) / \xi_{n}$ converges.

Finally, for $x, y \in \mathbb{R}^{d}$ we have

$$
\begin{aligned}
|f(x+i y)| & \leq(2 \pi)^{-d / 2} \int_{\mathbb{R}^{d}}\left|e^{-i\langle p, x+i y\rangle} \phi(p)\right| d p \\
& =(2 \pi)^{-d / 2} \int_{\mathbb{R}^{d}}\left|e^{\langle p, y\rangle} \phi(p)\right| d p \leq(2 \pi)^{-d / 2} \int_{\mathbb{R}^{d}} e^{|p||y|}|\phi(p)| d p \\
& \leq(2 \pi)^{-d / 2} e^{R|y|}\|\phi\|_{1} .
\end{aligned}
$$

10.6 Corollary. Let $f \in L^{2}\left(\mathbb{R}^{d}\right)$ such that its Fourier transform $\hat{f}$ vanishes almost everywhere outside the compact ball centered at the origin with radius $R>0$. Then there exists an entire function $F: \mathbb{C}^{d} \rightarrow \mathbb{C}$ such that $\left.F\right|_{\mathbb{R}^{d}}=f$ almost everywhere and

$$
|F(z)| \leq C e^{R|\operatorname{Im} z|} \quad \forall z \in \mathbb{C}^{d},
$$

for some constant $C>0$.

Proof. Let $\phi \in[\hat{f}]$ and let $\varphi(p):=\phi(-p)$ for $p \in \mathbb{R}^{d}$. Then $\varphi \in L^{2}\left(\mathbb{R}^{d}\right)$ and $\varphi$ vanishes almost everywhere outside the compact ball centered at the origin with radius $R>0$. Therefore, by Lemma $10.5, F: \mathbb{C}^{d} \rightarrow \mathbb{C}$,

$$
F(z):=(2 \pi)^{-d / 2} \int_{\mathbb{R}^{d}} e^{-i\langle p, z\rangle} \varphi(p) d p
$$

is entire with

$$
|F(z)| \leq C e^{R|\operatorname{Im} z|} \quad \forall z \in \mathbb{C}^{d},
$$

for some $C>0$. Since

$$
F(x)=(2 \pi)^{-d / 2} \int_{\mathbb{R}^{d}} e^{-i\langle p, x\rangle} \phi(-p) d p=(2 \pi)^{-d / 2} \int_{\mathbb{R}^{d}} e^{i\langle p, x\rangle} \hat{f}(p) d p
$$

and $\hat{f} \in L^{1}\left(\mathbb{R}^{d}\right) \cap L^{2}\left(\mathbb{R}^{d}\right)$, we have $\left.F\right|_{\mathbb{R}^{d}}=f$ almost everywhere. 
To state some important Corollaries of the Plancherel-Pólya Theorem we need two simple Lemmata.

10.7 Lemma. Let $C, R>0$ and let $f: \mathbb{C}^{d} \rightarrow \mathbb{C}$ be an entire function such that

$$
|f(z)| \leq C e^{R|z|} \quad \forall z \in \mathbb{C}^{d} .
$$

Then the P-indicator of $f$ satisfies the estimate

$$
h_{f}(\lambda) \leq R \quad \forall \lambda \in \mathbb{R}^{d} \text { with }|\lambda|=1 .
$$

Proof. Let $|\lambda|=1, x \in \mathbb{R}^{d}$. Then

$$
h_{f}(\lambda, x)=\limsup _{r \rightarrow \infty} \frac{\log |f(x+i \lambda r)|}{r} \leq \limsup _{r \rightarrow \infty} \frac{\log \left(C e^{R|x+i \lambda r|}\right)}{r} \leq R|\lambda|=R,
$$

thus $h_{f}(\lambda) \leq R$.

10.8 Lemma. Let $R>0$ and let $D \subset \mathbb{R}^{d}$ such that $H_{D}(\lambda) \leq R$ for all $\lambda \in \mathbb{R}^{d}$ with $|\lambda|=1$. Then

$$
D \subset\left\{x \in \mathbb{R}^{d}:|x| \leq R\right\} .
$$

Proof. Let $x_{0} \in D \backslash\{0\}$. Then for $\lambda:=x_{0} /\left|x_{0}\right|$ we have

$$
\left|x_{0}\right|=\left\langle\lambda, x_{0}\right\rangle \leq \sup _{x \in D}\langle\lambda, x\rangle=H_{D}(\lambda) \leq R .
$$

10.9 Corollary. Let $C, R>0$ and let $f: \mathbb{C}^{d} \rightarrow \mathbb{C}$ be an entire function such that $\left.f\right|_{\mathbb{R}^{d}} \in L^{2}\left(\mathbb{R}^{d}\right)$ and

$$
|f(z)| \leq C e^{R|z|} \quad \forall z \in \mathbb{C}^{d} .
$$

Then there exists a $\phi \in L^{2}\left(\mathbb{R}^{d}\right)$ which vanishes outside the compact ball centered at the origin with radius $R>0$, such that

$$
f(z)=(2 \pi)^{-d / 2} \int_{\mathbb{R}^{d}} e^{-i\langle p, z\rangle} \phi(p) d p \quad \forall z \in \mathbb{C}^{d} .
$$

Moreover,

$$
|f(z)| \leq C^{\prime} e^{R|\operatorname{Im} z|} \quad \forall z \in \mathbb{C}^{d}
$$

for some constant $C^{\prime}>0$.

Proof. By Theorem 10.4 there exists a $\phi \in L^{2}\left(\mathbb{R}^{d}\right)$ which vanishes outside some bounded set such that

$$
f(z)=(2 \pi)^{-d / 2} \int_{\mathbb{R}^{d}} e^{-i\langle p, z\rangle} \phi(p) d p \quad \forall z \in \mathbb{C}^{d} .
$$

Let $D$ be the smallest convex set in $\mathbb{R}^{d}$ such that $\left.\phi\right|_{D^{c}}=0$. Since $H_{D}=h_{f}$ and, by Lemma $10.7, h_{f}(\lambda) \leq R$ for all $|\lambda|=1$, Lemma 10.8 implies

$$
D \subset B:=\left\{x \in \mathbb{R}^{d}:|x| \leq R\right\} .
$$

Hence $\left.\phi\right|_{B^{c}}=0$. Now Lemma 10.5 completes the proof. 
10.10 Corollary. Let $R_{0}>0$ and let $f$ be an entire function on $\mathbb{C}^{d}$ such that $\left.f\right|_{\mathbb{R}^{d}} \in$ $L^{2}\left(\mathbb{R}^{d}\right)$. If for each $R>R_{0}$ there exists a $C_{R}>0$ such that

$$
|f(z)| \leq C_{R} e^{R|z|} \quad \forall z \in \mathbb{C}^{d},
$$

then

$$
|f(z)| \leq C e^{R_{0}|\operatorname{Im} z|} \quad \forall z \in \mathbb{C}^{d}
$$

for some constant $C>0$.

Proof. By Lemma 10.7 we have

$$
h_{f}(\lambda) \leq R \quad \forall R>R_{0} \quad \forall \lambda \in \mathbb{R}^{d} \text { with }|\lambda|=1 .
$$

Hence

$$
h_{f}(\lambda) \leq R_{0} \quad \forall \lambda \in \mathbb{R}^{d} \text { with }|\lambda|=1 .
$$

By Theorem 10.4 there exists a $\phi \in L^{2}\left(\mathbb{R}^{d}\right)$ which vanishes outside some bounded set such that

$$
f(z)=(2 \pi)^{-d / 2} \int_{\mathbb{R}^{d}} e^{-i\langle p, z\rangle} \phi(p) d p \quad \forall z \in \mathbb{C}^{d} .
$$

Let $D$ be the smallest convex set in $\mathbb{R}^{d}$ such that $\left.\phi\right|_{D^{c}}=0$. Since $H_{D}(\lambda)=h_{f}(\lambda) \leq R_{0}$ for all $|\lambda|=1$, Lemma 10.8 implies

$$
D \subset B:=\left\{x \in \mathbb{R}^{d}:|x| \leq R_{0}\right\} .
$$

Hence $\phi$ vanishes outside $B$. $\forall z \in \mathbb{C}^{d}$

By Lemma 10.5 there exists a constant $C>0$ such that $|f(z)| \leq C e^{R_{0}|\operatorname{Im} z|}$

Before we present a Paley-Wiener type Theorem for bounded measurable functions we need some Lemmata.

The following Lemma and its proof is adapted from [[Rud91] Exercise 7.15].

10.11 Lemma. Let $f: \mathbb{C}^{d} \rightarrow \mathbb{C}$ be an entire function, $N \in \mathbb{N}, r \geq 0$ and

$$
\begin{array}{ll}
|f(z)| \leq(1+|z|)^{N} e^{r|\operatorname{Im} z|} & \forall z \in \mathbb{C}^{d}, \\
|f(x)| \leq 1 & \forall x \in \mathbb{R}^{d} .
\end{array}
$$

Then we have

$$
|f(z)| \leq e^{r|\operatorname{Im} z|} \quad \forall z \in \mathbb{C}^{d} .
$$

Proof. Fix $z=x+i y \in \mathbb{C}^{d}$ and let $s>0$. Define $g_{s}:\{\lambda \in \mathbb{C}: \operatorname{Im} \lambda \geq 0\} \rightarrow \mathbb{C}$ as

$$
g_{s}(\lambda):=(1-i s \lambda)^{-N-1} e^{i r|y| \lambda} f(x+\lambda y)
$$

Choose $R>1$ such that $(1+|x|+R|y|)^{N}|s R-1|^{-N-1}<1$ and let

$$
\Omega:=\{\lambda \in \mathbb{C}:|\lambda| \leq R \text { and } \operatorname{Im} \lambda \geq 0\}
$$


Then $\left|g_{s}(\lambda)\right| \leq 1$ for all $\lambda \in \partial \Omega$. Indeed: For $\lambda \in[-R, R]$ we have

$$
\left|g_{s}(\lambda)\right| \leq|1-i s \lambda|^{-N-1}=\left(1+s^{2} \lambda^{2}\right)^{(-N-1) / 2} \leq 1,
$$

and for $\lambda=R e^{i \phi}, \phi \in[0, \pi]$, we have

$$
\left|g_{s}(\lambda)\right| \leq \frac{\left(1+\left|x+R e^{i \phi} y\right|\right)^{N}}{\left|1-i s R e^{i \phi}\right|^{N+1}} \underbrace{e^{-r|y| R \sin (\phi)} e^{r|R \sin (\phi) y|}}_{=1} .
$$

Since

$$
\left|x+R e^{i \phi} y\right| \leq|x|+R|y|
$$

and

$$
|s R-1|=|| i s R e^{i \phi}|-| 1|| \leq\left|1-i s R e^{i \phi}\right|,
$$

we have $\left|g_{s}(\lambda)\right| \leq 1$.

Then the Maximum Modulus Theorem (see, e.g., [[Con78] Theorem VI.1.4]) implies $\left|g_{s}(i)\right| \leq 1$. So we have

$$
|f(z)| \leq(1+s)^{N+1} e^{r|\operatorname{Im} z|} .
$$

Now $s \rightarrow 0$ completes the proof.

10.12 Corollary. Let $C, r \geq 0$ and let $f: \mathbb{C}^{d} \rightarrow \mathbb{C}$ be an entire function such that

$$
\begin{array}{ll}
|f(z)| \leq C e^{r|\operatorname{Im} z|} & \forall z \in \mathbb{C}^{d}, \\
|f(x)| \leq 1 & \forall x \in \mathbb{R}^{d} .
\end{array}
$$

Then we have

$$
|f(z)| \leq e^{r|\operatorname{Im} z|} \quad \forall z \in \mathbb{C}^{d}
$$

Proof. Let $\delta>1$. Then

$$
\frac{|f(x)|}{\delta}<1 \quad \forall x \in \mathbb{R}^{d}
$$

By continuity there exists an $R>0$ such that

$$
\frac{|f(z)|}{\delta}<1 \quad \forall|z| \leq R
$$

Choose an $N \in \mathbb{N}$ such that

$$
\frac{C}{\delta} \leq(1+|z|)^{N} \quad \forall|z|>R
$$

If we apply Lemma 10.11 to $\delta^{-1} f$ we obtain

$$
|f(z)| \leq \delta e^{r|\operatorname{Im} z|} \quad \forall z \in \mathbb{C}^{d} .
$$

Now $\delta \rightarrow 1$ completes the proof. 
10.13 Lemma. Let $\left(x_{n}\right)_{n \in \mathbb{N}}$ be an unbounded sequence in $\mathbb{R}$ and let $\alpha \in \mathbb{R} \backslash\{0\}$. Then there exists $a \xi \in(0,1)$ and a subsequence $\left(x_{n_{k}}\right)_{k \in \mathbb{N}}$ such that

$$
\left|\sin \left(\alpha \xi x_{n_{k}}\right)\right| \geq \frac{1}{2} \quad \forall k \in \mathbb{N} .
$$

Proof. It suffices to show the assertion for $\alpha=\pi$, since then for the unbounded sequence $\left(\tilde{x}_{n}\right)_{n \in \mathbb{N}}$, where $\tilde{x}_{n}:=\alpha x_{n} / \pi$ we have

$$
\left|\sin \left(\alpha \xi x_{n_{k}}\right)\right|=\left|\sin \left(\pi \xi \tilde{x}_{n_{k}}\right)\right| \geq \frac{1}{2} \quad \forall k \in \mathbb{N} .
$$

Without loss of generality we may assume that $x_{n}>1$ for all $n$. Define $T$ : $[0, \infty) \rightarrow[0,1]$ by

$$
T(x):= \begin{cases}x \bmod 1, & x \bmod 1 \in[0,1 / 2], \\ 1-(x \bmod 1), & x \bmod 1 \in(1 / 2,1] .\end{cases}
$$

Because $|\sin (\pi x)| \geq 2 T(x)$ for all $x \geq 0$, it suffices to show that there exists a $\xi \in(0,1)$ and a subsequence $\left(x_{n_{k}}\right)_{k}$ such that $T\left(\xi x_{n_{k}}\right) \geq 1 / 4$ for all $k$. Put $x_{n_{1}}:=x_{1}$ and

$$
I_{1}:=\left[\frac{1}{4 x_{n_{1}}}, \frac{3}{4 x_{n_{1}}}\right] \text {. }
$$

Then we have

$$
T\left(\xi x_{n_{1}}\right) \geq \frac{1}{4} \quad \forall \xi \in I_{1} .
$$

Now suppose $x_{n_{1}}, x_{n_{2}}, \ldots x_{n_{j}}$ and $I_{1} \supset I_{2} \supset \ldots \supset I_{j}$ are given, and that $I_{j}=\left[a_{j}, b_{j}\right]$, where $0<a_{j}<b_{j}<1$. Let $x_{n_{j+1}}$ be the first element in the sequence $\left(x_{n}\right)_{n}$ such that $n_{j+1}>n_{j}$ and $x_{n_{j+1}}>\frac{7 / 4}{b_{j}-a_{j}}$. Put

$$
I_{j+1}:=\left[\frac{\left\lceil a_{j} x_{n_{j+1}}\right\rceil}{x_{n_{j+1}}}+\frac{1}{4 x_{n_{j+1}}}, \frac{\left\lceil a_{j} x_{n_{j+1}}\right\rceil}{x_{n_{j+1}}}+\frac{3}{4 x_{n_{j+1}}}\right],
$$

where $\lceil x\rceil$ is the smallest integer which is greater than or equal to $x$. It is easy to see that $I_{j+1} \subset I_{j}$ and that we have $T\left(\xi x_{n_{j+1}}\right) \geq 1 / 4$ for all $\xi \in I_{j+1}$. Hence we obtain a subsequence $\left(x_{n_{k}}\right)_{k}$ and a sequence of compact sets $\left(I_{k}\right)_{k}$ such that $I_{k+1} \subset I_{k} \neq \varnothing$ for all $k$, whence there exists a $\xi \in \cap_{j \in \mathbb{N}} I_{j}$ such that $T\left(\xi x_{n_{k}}\right) \geq 1 / 4$ for all $k$.

10.14 Lemma. Let $r>0$. Then there exists a constant $C>0$ such that for each $z \in \mathbb{C}$ there exists an $\varepsilon \in(0, r]$ with $s(\varepsilon z) \neq 0$ and

$$
\frac{1}{|s(\varepsilon z)|} \leq C(1+|z|) e^{-r|\operatorname{Im} z|},
$$

where $s: \mathbb{C} \rightarrow \mathbb{C}, s(z):=\sin (z) / z$ if $z \neq 0$ and $s(0):=1$. 
Proof. Let $z=x+i y$. If $y=0$ choose $\varepsilon \in(0, r]$ such that $|\varepsilon x| \leq 1$. Then $C:=\sin (1)^{-1}$ satisfies the estimate. It thus remains to show the assertion for all $z \in \mathbb{C} \backslash \mathbb{R}$. Note that $\sin (z)=0$ if and only if $z \in \pi \mathbb{Z}$. If the assertion fails, then for every $n \in \mathbb{N}$ exists a $z_{n} \in \mathbb{C} \backslash \mathbb{R}$ such that

$$
\frac{\left|\varepsilon z_{n}\right|}{\left|\sin \left(\varepsilon z_{n}\right)\right|}>n\left(1+\left|z_{n}\right|\right) e^{-r\left|\operatorname{Im} z_{n}\right|} \quad \forall n \in \mathbb{N} \quad \forall \varepsilon \in(0, r] .
$$

Then the sequence $\left(z_{n}\right)_{n \in \mathbb{N}}$ must be unbounded, since otherwise there exists an $\varepsilon \in(0, r]$ such that $\left|\varepsilon z_{n}\right| \leq \pi / 2$ for all $n \in \mathbb{N}$, and the left-hand side of $(\star)$ is bounded but the right-hand side is not, which is a contradiction. we have

For each $n \in \mathbb{N}$ let $z_{n}=x_{n}+i y_{n}$. Since $\left|\sin \left(\varepsilon z_{n}\right)\right|^{2}=\frac{1}{2}\left(\cosh \left(2 \varepsilon y_{n}\right)-\cos \left(2 \varepsilon x_{n}\right)\right)$,

$$
\varepsilon^{2}>\frac{n^{2}}{2}\left(\frac{1+\left|z_{n}\right|}{\left|z_{n}\right|}\right)^{2} e^{-2 r\left|y_{n}\right|}\left(\cosh \left(2 \varepsilon y_{n}\right)-\cos \left(2 \varepsilon x_{n}\right)\right) \quad \forall n \in \mathbb{N} \quad \forall \varepsilon \in(0, r] .
$$

Suppose $\left(y_{n}\right)_{n \in \mathbb{N}}$ is bounded. In that case $\left(x_{n}\right)_{n \in \mathbb{N}}$ is unbounded. The estimate

$$
\cosh (2 \varepsilon y)-\cos (2 \varepsilon x) \geq 1-\cos (2 \varepsilon x)=2 \sin ^{2}(\varepsilon x) \quad \forall x, y \in \mathbb{R}
$$

implies

$$
\varepsilon^{2}>n^{2} e^{-2 r\left|y_{n}\right|} \sin ^{2}\left(\varepsilon x_{n}\right) \geq D n^{2} \sin ^{2}\left(\varepsilon x_{n}\right) \quad \forall n \in \mathbb{N} \quad \forall \varepsilon \in(0, r],
$$

for some constant $D>0$. But by Lemma 10.13 there exists an $\varepsilon_{*} \in(0, r]$ and a subsequence $\left(x_{n_{k}}\right)_{k \in \mathbb{N}}$ such that $\sin ^{2}\left(\varepsilon_{*} x_{n_{k}}\right) \geq 1 / 4$ for all $k \in \mathbb{N}$, and thus

$$
\varepsilon_{*}^{2}>\frac{D}{4} n_{k}^{2} \quad \forall k \in \mathbb{N},
$$

which is impossible. Thus $\left(y_{n}\right)_{n \in \mathbb{N}}$ is unbounded. Since

$$
\cosh (2 \varepsilon y)-\cos (2 \varepsilon x) \geq \cosh (2 \varepsilon y)-1=2 \sinh ^{2}(\varepsilon y) \quad \forall x, y \in \mathbb{R},
$$

we have

$$
\varepsilon^{2}>n^{2} e^{-2 r\left|y_{n}\right|} \sinh ^{2}\left(\varepsilon y_{n}\right) \quad \forall n \in \mathbb{N} \quad \forall \varepsilon \in(0, r] .
$$

which is impossible, because $e^{-2 r|y|} \sinh ^{2}(r y)>\frac{1}{16}$ for all large enough $|y|$.

We are now ready to prove the following Paley-Wiener type Theorem for bounded matrix multiplication operators. It is strongly motivated by [[Cas84] Lemma 2]. The Theorem must not be confused with Schwartz's Paley-Wiener Theorem for distributions.

10.15 Theorem. For a measurable and bounded matrix-valued function $A: \mathbb{R}^{d} \rightarrow$ $\mathbb{C}^{m \times m}$ let $T_{A}$ be the bounded linear operator on $L^{2}\left(\mathbb{R}^{d}, \mathbb{C}^{m}\right)$ given by

$$
T_{A}[g]:=\mathscr{F}^{-1} A \mathscr{F}[g],
$$


where $\mathscr{F}$ denotes the Fourier transform. For $R>0$ let $E_{R}$ denote the multiplication operator on $L^{2}\left(\mathbb{R}^{d}, \mathbb{C}^{m}\right)$ given by

$$
E_{R}[g]:=\left[\chi_{B_{R}(0)} g\right]
$$

where $\chi_{B_{R}(0)}$ is the characteristic function of $B_{R}(0):=\left\{x \in \mathbb{R}^{d}:|x|<R\right\}$.

(a) Let $R>0$. If there exists an $R^{\prime}>R$ such that $\left(I-E_{R^{\prime}}\right) T_{A} E_{R}=0$, then there exists an entire function $\Phi: \mathbb{C}^{d} \rightarrow \mathbb{C}^{m \times m}$ such that $\left.\Phi\right|_{\mathbb{R}^{d}}=A$ almost everywhere and

$$
\|\Phi(z)\| \leq\left\|\left.\Phi\right|_{\mathbb{R}^{d}}\right\|_{\infty} e^{\left(R^{\prime}-R\right)|\operatorname{Im} z|} \quad \forall z \in \mathbb{C}^{d} .
$$

(b) Let $\delta>0$ and let $\Phi: \mathbb{C}^{d} \rightarrow \mathbb{C}^{m \times m}$ be an entire function such that $\left.\Phi\right|_{\mathbb{R}^{d}}$ is bounded and

$$
\|\Phi(z)\| \leq C e^{\delta|z|} \quad \forall z \in \mathbb{C}^{d}
$$

for some constant $C>0$. Then $A:=\left.\Phi\right|_{\mathbb{R}^{d}}$ satisfies $\left(I-E_{R+\delta}\right) T_{A} E_{R}=0$ for all $R>0$.

Proof. (a) Let us first consider the case $m=1$.

Let $Q_{r, c}:=\left[c_{1}-r_{1}, c_{1}+r_{1}\right] \times \ldots \times\left[c_{d}-r_{d}, c_{d}+r_{d}\right]$ be the cuboid centered at $c \in \mathbb{R}^{d}$ with edge lengths $2 r_{1}, \ldots, 2 r_{d}>0$ and sides parallel to the axes. The characteristic function of $Q_{r, c}$, denoted by $q_{r, c}$, vanishes outside a bounded region, therefore its Fourier-Laplace transform $g_{r, c}$ is an exponentially bounded entire function given by

$$
g_{r, c}(z)=(2 \pi)^{-d / 2} \int_{\mathbb{R}^{d}} e^{-i\langle p, z\rangle} q_{r, c}(p) d p=K_{r} e^{-i\langle c, z\rangle} \prod_{k=1}^{d} s\left(r_{k} z_{k}\right) \quad \forall z \in \mathbb{C}^{d},
$$

where $K_{r}:=(2 \pi)^{-d / 2} 2^{d}\left(r_{1} \cdot \ldots \cdot r_{d}\right)$ and $s: \mathbb{C} \rightarrow \mathbb{C}, s(z):=\sin (z) / z$ for $z \neq 0$ and $s(0):=1$.

Let $|c|<R$ and choose $r$ such that $q_{r, c}$ vanishes outside of $B_{R}(0)$. Then by the assumption there exists an $R^{\prime}>R$ such that

$$
0=\left(I-E_{R^{\prime}}\right) T_{A} E_{R} q_{r, c}=\left(I-E_{R^{\prime}}\right) T_{A} q_{r, c} .
$$

Thus $T_{A} q_{r, c}$ vanishes almost everywhere outside the compact ball of radius $R^{\prime}$ centered at the origin. By Lemma 10.5

$$
h_{r, c}(z):=(2 \pi)^{-d / 2} \int_{\mathbb{R}^{d}} e^{-i\langle p, z\rangle}\left(T_{A} q_{r, c}\right)(p) d p
$$

is an exponentially bounded entire function satisfying

$$
\left|h_{r, c}(z)\right| \leq C_{r, c} e^{R^{\prime}|\operatorname{Im} z|} \quad \forall z \in \mathbb{C}^{d},
$$

for some constant $C_{r, c}$. Since

$$
\left[\left.h_{r, c}\right|_{\mathbb{R}^{d}}\right]=\mathscr{F} T_{A}\left[q_{r, c}\right]=\mathscr{F} \mathscr{F}^{-1} A \mathscr{F}\left[q_{r, c}\right]=\left[\left.A g_{r, c}\right|_{\mathbb{R}^{d}}\right],
$$


we have $\left.h_{r, c}\right|_{\mathbb{R}^{d}}=\left.A g_{r, c}\right|_{\mathbb{R}^{d}}$ almost everywhere.

To show that $A$ has an entire extension let

$$
c=0, \quad r(\alpha):=(\alpha, \ldots, \alpha), \quad g_{\alpha}:=g_{r(\alpha), 0}, \quad h_{\alpha}:=h_{r(\alpha), 0},
$$

where $\alpha \in(0, R / \sqrt{d})$, and let $N_{\alpha} \subset \mathbb{C}^{d}$ denote the set of zeros of $g_{\alpha}$. Note that $g_{\alpha}(z)=0$ if and only if $z_{k} \in \pi \alpha^{-1} \mathbb{Z} \backslash\{0\}$ for at least one $k \in\{1, \ldots, d\}$. For every $\alpha, \alpha^{\prime} \in(0, R / \sqrt{d})$ it is

$$
\left.\left.g_{\alpha^{\prime}} h_{\alpha}\right|_{\mathbb{R}^{d}} \stackrel{\text { a.e. }}{=} g_{\alpha^{\prime}} A g_{\alpha}\right|_{\mathbb{R}^{d}}=\left.\left.g_{\alpha} A g_{\alpha^{\prime}}\right|_{\mathbb{R}^{d}} \stackrel{\text { a.e. }}{=} g_{\alpha} h_{\alpha^{\prime}}\right|_{\mathbb{R}^{d}} .
$$

Because every continuous function on $\mathbb{R}^{d}$ which vanishes almost everywhere is identically zero, we obtain

$$
\left.g_{\alpha^{\prime}} h_{\alpha}\right|_{\mathbb{R}^{d}}=\left.g_{\alpha} h_{\alpha^{\prime}}\right|_{\mathbb{R}^{d}} .
$$

Moreover, since both sides of this equation consist of entire functions (restricted to the real numbers), we must have

$$
g_{\alpha^{\prime}}(z) h_{\alpha}(z)=g_{\alpha}(z) h_{\alpha^{\prime}}(z) \quad \forall z \in \mathbb{C}^{d}
$$

(see [[Die69] (9.4.4)]). Hence,

$$
\Phi_{\alpha}(z):=\frac{h_{\alpha}(z)}{g_{\alpha}(z)}
$$

is almost everywhere an analytic extension of $A$ for $|z|<\pi / \alpha$, and for these $z$ it is $\Phi_{\alpha}(z)=\Phi_{\alpha^{\prime}}(z)$ for all $\alpha^{\prime} \in(0, \alpha)$. By letting $\alpha$ tend to zero we obtain an entire function $\Phi: \mathbb{C}^{d} \rightarrow \mathbb{C}$ such that $\left.\Phi\right|_{\mathbb{R}^{d}}=A$ almost everywhere. Note that, since every continuous function that is bounded almost everywhere is bounded, we have $|\Phi(x)| \leq\left\|\left.\Phi\right|_{\mathbb{R}^{d}}\right\|_{\infty}$ for all $x \in \mathbb{R}^{d}$.

To prove that $\Phi$ is exponentially bounded let $|c|<R$. Then $Q_{r, c} \subset B_{R}(0)$ as long as $|c|+\sqrt{d} r_{k}<R$ for all $k \in\{1, \ldots, d\}$. We still have

$$
\left|h_{r, c}(z)\right| \leq C_{r, c} e^{R^{\prime}|\operatorname{Im} z|} \quad \forall z \in \mathbb{C}^{d},
$$

for some constant $C_{r, c}$. Since $h_{r, c}=\Phi g_{r, c}$, we find

$$
\left|h_{r, c}(x)\right|=|\Phi(x)|\left|g_{r, c}(x)\right| \leq\left\|\left.\Phi\right|_{\mathbb{R}^{d}}\right\|_{\infty} K_{r} \quad \forall x \in \mathbb{R}^{d},
$$

and using Corollary 10.12 we obtain the estimate

$$
\left|h_{r, c}(z)\right| \leq\left\|\left.\Phi\right|_{\mathbb{R}^{d}}\right\|_{\infty} K_{r} e^{R^{\prime}|\operatorname{Im} z|} \quad \forall z \in \mathbb{C}^{d} .
$$

Hence, for all $r, c$ satisfying $Q_{r, c} \subset B_{R}(0)$ we have

$$
|\Phi(z)| \prod_{k=1}^{d}\left|s\left(r_{k} z_{k}\right)\right| \leq\left\|\left.\Phi\right|_{\mathbb{R}^{d}}\right\|_{\infty} e^{-\langle c, \operatorname{Im} z\rangle} e^{R^{\prime}|\operatorname{Im} z|} \quad \forall z \in \mathbb{C}^{d}
$$


Let $\delta \in(0, R)$ and let $y:=\operatorname{Im} z \neq 0$. Put $c:=(R-\delta) y /|y|$. Then $|c|=R-\delta$, $\langle c, y\rangle=(R-\delta)|y|$ and Eq. 10.3 implies that

$$
|\Phi(z)| \prod_{k=1}^{d}\left|s\left(r_{k} z_{k}\right)\right| \leq\left\|\left.\Phi\right|_{\mathbb{R}^{d}}\right\|_{\infty} e^{\left(R^{\prime}-R+\delta\right)|\operatorname{Im} z|} \quad \forall z \in \mathbb{C}^{d} \text { with } \operatorname{Im} z \neq 0
$$

holds for all $r_{k}$ satisfying $0<r_{k} \leq \delta / \sqrt{d}$. By continuity the estimate holds true for all $z \in \mathbb{C}^{d}$. From Lemma 10.14 we obtain constants $C_{k}>0$ such that for each $z \in \mathbb{C}^{d}$ there exists an $r \in(0, \delta / \sqrt{d}]^{d}$ with $s\left(r_{k} z_{k}\right) \neq 0$ and

$$
\frac{1}{\left|s\left(r_{k} z_{k}\right)\right|} \leq C_{k}\left(1+\left|z_{k}\right|\right) e^{-\delta\left|y_{k}\right| / \sqrt{d}} \quad(1 \leq k \leq d) .
$$

Therefore

$$
|\Phi(z)| \leq\left\|\left.\Phi\right|_{\mathbb{R}^{d}}\right\|_{\infty} C_{\delta}(1+|z|)^{d} e^{\left(R^{\prime}-R+\delta\right)|y|} e^{-\delta|y|_{1} / \sqrt{d}} \quad \forall z \in \mathbb{C}^{d},
$$

for some constant $C_{\delta}>0$. Since $|y| \leq|y|_{1}$, we have

$$
|\Phi(z)| \leq\left\|\left.\Phi\right|_{\mathbb{R}^{d}}\right\|_{\infty} C_{\delta}(1+|z|)^{d} e^{\left(R^{\prime}-R+\delta \kappa\right)|\operatorname{Im} z|} \quad \forall z \in \mathbb{C}^{d},
$$

where $\kappa:=1-1 / \sqrt{d}$. From Lemma 10.11 and Corollary 10.12 we find

$$
|\Phi(z)| \leq\left\|\left.\Phi\right|_{\mathbb{R}^{d}}\right\|_{\infty} e^{\left(R^{\prime}-R+\delta \kappa\right)|\operatorname{Im} z|} \quad \forall z \in \mathbb{C}^{d} .
$$

Now $\delta \rightarrow 0$ proves (a) for $m=1$.

The case $m>1$ : For $u, v \in \mathbb{C}^{m}$ with $|u|=|v|=1$ put $A_{u, v}: \mathbb{R}^{d} \rightarrow \mathbb{C}$, $A_{u, v}(x):=\langle u, A(x) v\rangle$. Then for every $g \in L^{2}\left(\mathbb{R}^{d}\right)$ we have

$$
0=\left\langle u,\left(I-E_{R^{\prime}}\right) T_{A} E_{R}[v g]\right\rangle=\left(I-E_{R^{\prime}}\right) T_{A_{u, v}} E_{R}[g] .
$$

Thus the case $m=1$ applies to $A_{u, v}$. If we let $u, v$ be standard basis vectors, we see that for every matrix element $A_{i j}$ there exists an entire function $\Phi_{i j}$ such that $\left.\Phi_{i j}\right|_{\mathbb{R}^{d}}=A_{i j}$ almost everywhere. Thus if $\Phi$ is the matrix-valued function with matrix elements $\Phi_{i j}$ then $\left.\Phi\right|_{\mathbb{R}^{d}}=A$ almost everywhere, since a finite union of null sets is a null set. Moreover, we have

$$
|\langle u, \Phi(z) v\rangle| \leq\left\|\left(\left.\Phi\right|_{\mathbb{R}^{d}}\right)_{u, v}\right\|_{\infty} e^{\left(R^{\prime}-R\right)|\operatorname{Im} z|} \quad \forall z \in \mathbb{C}^{d} .
$$

Since

$$
\left\|\left(\left.\Phi\right|_{\mathbb{R}^{d}}\right)_{u, v}\right\|_{\infty}=\sup _{x \in \mathbb{R}^{d}}|\langle u, \Phi(x) v\rangle| \leq\left\|\left.\Phi\right|_{\mathbb{R}^{d}}\right\|_{\infty}
$$

we have

$$
|\langle u, \Phi(z) v\rangle| \leq\left\|\left.\Phi\right|_{\mathbb{R}^{d}}\right\|_{\infty} e^{\left(R^{\prime}-R\right)|\operatorname{Im} z|} \quad \forall z \in \mathbb{C}^{d},
$$

for all $u, v$ with $|u|=|v|=1$. Fix $z \in \mathbb{C}^{d}$ and let $|v|=1$. If $\Phi(z) v \neq 0$ put $w:=|\Phi(z) v|^{-1} \Phi(z) v$, then we have

$$
|\Phi(z) v|=|\langle w, \Phi(z) v\rangle| \leq\left\|\left.\Phi\right|_{\mathbb{R}^{d}}\right\|_{\infty} e^{\left(R^{\prime}-R\right)|\operatorname{Im} z|} .
$$


It follows

$$
\|\Phi(z)\| \leq\left\|\left.\Phi\right|_{\mathbb{R}^{d}}\right\|_{\infty} e^{\left(R^{\prime}-R\right)|\operatorname{Im} z|}
$$

(b) Let us first consider the case $m=1$. Let $[g] \in L^{2}\left(\mathbb{R}^{d}\right)$ and $R>0$. Then $\phi:=E_{R} g$ vanishes outside the compact ball of radius $R$ centered at the origin. Hence,

$$
h(z):=(2 \pi)^{-d / 2} \int_{\mathbb{R}^{d}} e^{-i\langle p, z\rangle} \phi(p) d p
$$

is an entire function satisfying $\left.h\right|_{\mathbb{R}^{d}}=\mathscr{F} \phi$ almost everywhere and

$$
|h(z)| \leq C_{h} e^{R|\operatorname{Im} z|} \quad \forall z \in \mathbb{C}^{d},
$$

for some constant $C_{h}>0$. Thus

$$
|\Phi(z) h(z)| \leq C C_{h} e^{(R+\delta)|z|} \quad \forall z \in \mathbb{C}^{d} .
$$

Since $\left.\Phi h\right|_{\mathbb{R}^{d}} \in L^{2}$, Corollary 10.9 implies that there exists a $\tilde{g}$ which vanishes outside the compact ball of radius $R+\delta$ centered at the origin such that $\mathscr{F} \tilde{g}=\left.A h\right|_{\mathbb{R}^{d}}$ almost everywhere. Because $[\tilde{g}]=\mathscr{F}^{-1} A \mathscr{F} E_{R}[g]$, it follows that $\left(I-E_{R+\delta}\right) T_{A} E_{R}[g]=0$.

The case $m>1$ : Since $\left|\Phi_{i j}(z)\right| \leq\|\Phi(z)\|$ for all $z \in \mathbb{C}^{d}$, the case $m=1$ applies to $\Phi_{i j}(z)$. Thus for $f=\left(f_{1}, \ldots, f_{m}\right) \in L^{2}\left(\mathbb{R}^{d}, \mathbb{C}^{m}\right)$ we have

$$
\left\langle e_{i},\left(I-E_{R+\delta}\right) T_{A} E_{R}[f]\right\rangle=\sum_{j=1}^{d}\left(I-E_{R+\delta}\right) T_{A_{i j}} E_{R}\left[f_{j}\right]=0 \quad \forall 1 \leq i \leq m .
$$

This completes the proof.

If we combine both parts of the Theorem we obtain two important Corollaries. 10.16 Corollary. Let $R>0$ and let $A: \mathbb{R}^{d} \rightarrow \mathbb{C}^{m \times m}$ be a bounded measurable matrixvalued function such that $\left(I-E_{R^{\prime}}\right) T_{A} E_{R}=0$ for some $R^{\prime}>R$. Then

$$
\left(I-E_{S+\delta}\right) T_{A} E_{S}=0 \quad \forall S>0
$$

where $\delta:=R^{\prime}-R$.

Proof. Apply Theorem 10.15 (a) and then (b).

10.17 Corollary. Let $\Phi: \mathbb{C}^{d} \rightarrow \mathbb{C}^{m \times m}$ be an entire matrix-valued function such that $\left.\Phi\right|_{\mathbb{R}^{d}}$ is bounded and

$$
\|\Phi(z)\| \leq C e^{\delta|z|} \quad \forall z \in \mathbb{C}^{d},
$$

for some constants $C, \delta>0$. Then

$$
\|\Phi(z)\| \leq\left\|\left.\Phi\right|_{\mathbb{R}^{d}}\right\|_{\infty} e^{\delta|\operatorname{Im} z|} \quad \forall z \in \mathbb{C}^{d} .
$$


Proof. Put $A:=\left.\Phi\right|_{\mathbb{R}^{d}}$. Theorem 10.15 (b) implies that $\left(I-E_{R+\delta}\right) T_{A} E_{R}=0$ for all $R>0$. By part (a) of the same Theroem there exists an entire function $\tilde{\Phi}: \mathbb{C}^{d} \rightarrow \mathbb{C}$ such that $\left.\tilde{\Phi}\right|_{\mathbb{R}^{d}}=A$ almost everywhere and

$$
\|\tilde{\Phi}(z)\| \leq\left\|\left.\tilde{\Phi}\right|_{\mathbb{R}^{d}}\right\|_{\infty} e^{\delta|\operatorname{Im} z|} \quad \forall z \in \mathbb{C}^{d} .
$$

Since every continuous function on $\mathbb{R}^{d}$ which vanishes almost everywhere is identically zero, we must have $\left.\tilde{\Phi}\right|_{\mathbb{R}^{d}}=\left.\Phi\right|_{\mathbb{R}^{d}}$. Hence, $\tilde{\Phi}$ and $\Phi$ must be identical (see [[Die69] $(9.4 .4)])$. 


\section{Unitary One-Parameter Groups of Matrix Mul- tiplication Operators}

11.1. Motivation. Let $(V, U, E)$ be the coordinate space representation of a finite causal localization. From Theorem 2.14 we have that in the helicity representation $V^{h}(t)[f]=\left[\Psi_{t}(|\cdot|) f\right]$, where for each $t \in \mathbb{R}, \Psi_{t}: \mathbb{C} \rightarrow L\left(\oplus_{j} \nu_{j} \mathbb{C}^{2 j+1}\right)$ is an exponentially bounded entire matrix-valued function. Then Stone's Theorem leads us to the conjecture that $\Psi_{t}=e^{i t h}$ for some entire matrix-valued function $h$ such that $h(\rho)$ is self-adjoint for all $\rho>0$. That this is indeed the case is the subject of Theorem 11.10. which is needed in the proof of Theorem 2.17 .

11.2. Notation. In this section $\lambda$ will always denote the Lebesgue measure on $\mathbb{R}^{d}$. Also, when no confusion is possible, we will abbreviate $L^{2}\left(\mathbb{R}^{d}, \mathbb{C}^{m}, \lambda\right)$ by $L^{2}$.

11.3 Lemma. Let $H$ be a densely defined self-adjoint linear operator in $L^{2}\left(\mathbb{R}^{d}, \mathbb{C}^{m}, \lambda\right)$ such that for all $[f] \in \mathscr{D}(H)$,

$$
H[f]=[h f], \quad(h f)(p):=h(p) f(p),
$$

where $h: \mathbb{R}^{d} \rightarrow \mathbb{C}^{m \times m}$ is a measurable matrix-valued function. Then $h^{*}=h \lambda$-a.e. and the domain of $H$ is

$$
\mathscr{D}(H)=\left\{[f] \in L^{2}\left(\mathbb{R}^{d}, \mathbb{C}^{m}, \lambda\right):[h f] \in L^{2}\left(\mathbb{R}^{d}, \mathbb{C}^{m}, \lambda\right)\right\} .
$$

Proof. Consider the operator $G$ in $L^{2}, G[f]:=[h f]$ with domain

$$
\mathscr{D}(G):=\left\{[f] \in L^{2}:[h f] \in L^{2}\right\} .
$$

Obviously, $H \subset G$. Since $H$ is densely defined, so is $G$, thus $G^{*} \subset H^{*}=H$.

We show that $G$ is closed. For $(f, g) \in \overline{\operatorname{graph}(G)}$ let $\left(f_{n}, G f_{n}\right)_{n \in \mathbb{N}}$ be a sequence in $\operatorname{graph}(G)$ converging to $(f, g)$. After having chosen a suitable subsequence, $\left(f_{n(k)}\right)_{k \in \mathbb{N}}$ and $\left(h f_{n(k)}\right)_{k \in \mathbb{N}}$ converge pointwise almost everywhere to $f$ and $g$, respectively. Since $\left(h f_{n(k)}\right)_{k \in \mathbb{N}}$ also converges pointwise almost everywhere to $h f$, we have that $g$ equals $h f$ almost everywhere, hence $f \in \mathscr{D}(G)$ and $G f=g$. Thus $G$ is closed.

This implies (cf., e.g., Theorem 13.12 in Rud91) that $\mathscr{D}\left(G^{*}\right)$ is dense. For $f \in \mathscr{D}\left(G^{*}\right)$ and $g \in \mathscr{D}(G)$ we have $\langle f, G g\rangle=\left\langle G^{*} f, g\right\rangle=\langle H f, g\rangle$, thus

$$
\int_{\mathbb{R}^{d}}\left\langle f(p),\left(h^{*}(p)-h(p)\right) g(p)\right\rangle d \lambda(p)=0 .
$$

For $n \in \mathbb{N}$ put $g_{k}^{(n)}:=\xi_{n} b_{k}$, where $\left\{b_{1}, \ldots, b_{m}\right\}$ is some basis for $\mathbb{C}^{m}$ and $\xi_{n}$ is the characteristic function of

$$
A_{n}:=\left\{p \in \mathbb{R}^{d}:\|h(p)\|<n \text { and }|p|<n\right\} .
$$


Note that $g_{k}^{(n)}, h g_{k}^{(n)}$ and $h^{*} g_{k}^{(n)}$ are $L^{2}$ functions, in particular $g_{k}^{(n)} \in \mathscr{D}(G)$. Hence

$$
\left\langle f,\left(h^{*}-h\right) g_{k}^{(n)}\right\rangle=0 \quad \forall f \in \mathscr{D}\left(G^{*}\right) .
$$

Since $\mathscr{D}\left(G^{*}\right)$ is dense, this implies that for each $k \in\{1, \ldots, m\}$ and each $n \in \mathbb{N}$ there exists a set $N_{k, n}$ of measure zero such that

$$
\left(h^{*}(p)-h(p)\right) g_{k}^{(n)}(p)=0 \quad \forall p \in \mathbb{R}^{d} \backslash N_{k, n} .
$$

Let $N$ be the union of all $N_{k, n}$. Then $N$, being a countable union of null sets, is itself a set of measure zero. If $p \in \mathbb{R}^{d} \backslash N$, choose $n \in \mathbb{N}$ such that $p \in A_{n}$. So we have $\left(h^{*}(p)-h(p)\right) b_{k}=0$ for all $k$. Hence $h^{*}=h \lambda$-a.e.

Finally, let $g \in \mathscr{D}(G)$. Then for all $f \in \mathscr{D}(H)$ we have

$$
\langle H f, g\rangle=\int_{\mathbb{R}^{d}}\langle h(p) f(p), g(p)\rangle d \lambda(p)=\int_{\mathbb{R}^{d}}\langle f(p), h(p) g(p)\rangle d \lambda(p)=\langle f, G g\rangle,
$$

hence $g \in \mathscr{D}\left(H^{*}\right)=\mathscr{D}(H)$, whence $H=G$.

11.4 Lemma. Let $h: \mathbb{R}^{d} \rightarrow \mathbb{C}^{m \times m}$ be a measurable self-adjoint matrix-valued function and let $\mathscr{H}:=L^{2}\left(\mathbb{R}^{d}, \mathbb{C}^{m \times m}, \lambda\right)$. Then $W: \mathbb{R} \rightarrow L(\mathscr{H}), W(t)[f]:=\left[e^{i t h(\cdot)} f\right]$ defines a continuous one-parameter unitary group on $\mathscr{H}$.

Proof. For each $t, W(t)$ is a well-defined linear bounded operator, and it is easy to see that $W(t)$ is unitary. Let $[f] \in L^{2}$ and let $t, r \in \mathbb{R}$. Then

$$
W(t)(W(r)[f])=\left[p \mapsto e^{i t h(p)} e^{i r h(p)} f(p)\right]=\left[p \mapsto e^{i(t+r) h(p)} f(p)\right]=W(t+r)[f] .
$$

Hence $W(t) W(r)=W(t+r)$ for all $t, r \in \mathbb{R}$. It remains to show that $W$ is continuous. Let $t_{n} \stackrel{n \rightarrow \infty}{\longrightarrow} 0$. Obviously the integrand in

$$
\left\|\left(W\left(t_{n}\right)-I\right) f\right\|^{2}=\int_{\mathbb{R}^{d}}\left\|\left(e^{i t_{n} h(p)}-I_{m}\right) f(p)\right\|^{2} d \lambda(p),
$$

vanishes pointwisely for $n \rightarrow \infty$, and $4\|f(\cdot)\|^{2}$ is an integrable dominating function. By Lebesgue's Theorem of Dominated Convergence $\left\|\left(W\left(t_{n}\right)-I\right) f\right\| \stackrel{n \rightarrow \infty}{\longrightarrow} 0$.

11.5 Proposition. Let $V$ be a continuous one-parameter unitary group on $L^{2}\left(\mathbb{R}^{d}, \mathbb{C}^{m}, \lambda\right)$ and suppose that for each $t \in \mathbb{R}$ there exists a measurable matrix-valued function $v_{t}: \mathbb{R}^{d} \rightarrow \mathbb{C}^{m \times m}$ such that $V(t)[f]=\left[v_{t} f\right]$ for all $[f] \in L^{2}\left(\mathbb{R}^{d}, \mathbb{C}^{m}, \lambda\right)$. Then there exists a measurable self-adjoint matrix-valued function $h: \mathbb{R}^{d} \rightarrow \mathbb{C}^{m \times m}$ such that for every $t \in \mathbb{R}$,

$$
v_{t}(p)=\exp (i t h(p)) \quad \lambda \text {-a.e. }
$$

In particular, $v_{t}(p)$ is unitary $\lambda$-a.e. Moreover, if Eq. (11.1) holds, then the generator $H$ for $V$ has the domain

$$
\mathscr{D}(H)=\left\{[f] \in L^{2}\left(\mathbb{R}^{d}, \mathbb{C}^{m}, \lambda\right):[h f] \in L^{2}\left(\mathbb{R}^{d}, \mathbb{C}^{m}, \lambda\right)\right\}
$$

and satisfies $H[f]=[h f]$ for every $[f]$ in $\mathscr{D}(H)$. 
Proof. By Stone's Theorem (cf., e.g., Theorem 13.38 in [Rud91]) there exists a selfadjoint operator $H$ in $L^{2}$ such that

$$
\left\|\frac{V(t)[f]-[f]}{t}-i H[f]\right\| \stackrel{t \rightarrow 0}{\longrightarrow} 0 \quad \forall[f] \in \mathscr{D}(H) .
$$

Thus if $\left(t_{n}\right)_{n \in \mathbb{N}}$ is a sequence with $0 \neq t_{n} \stackrel{n \rightarrow \infty}{\longrightarrow} 0$, and $[f] \in \mathscr{D}(H)$ then

$$
\int_{\mathbb{R}^{d}}\left\|\frac{v_{t_{n}}(p) f(p)-f(p)}{t_{n}}-i(H f)(p)\right\|^{2} d \lambda(p) \stackrel{n \rightarrow \infty}{\longrightarrow} 0 .
$$

Since $\operatorname{graph}(H)$ is a subspace of a separable metric space, it is separable, so there exists a countable set of functions $A:=\left\{f_{1}, f_{2}, \ldots\right\}$ such that $\left\{\left[f_{1}\right],\left[f_{2}\right], \ldots\right\} \subset \mathscr{D}(H)$ and $\left\{\left(\left[f_{k}\right], H\left[f_{k}\right]\right): k \in \mathbb{N}\right\}$ is dense in $\operatorname{graph}(H)$.

By the Riesz-Fischer Theorem, applied for $f_{1}$, there exists a subsequence $\left(t_{n}^{(1)}\right)_{n \in \mathbb{N}}$ of $\left(t_{n}\right)_{n \in \mathbb{N}}$ such that

$$
\frac{v_{t_{n}^{(1)}}(p) f_{1}(p)-f_{1}(p)}{t_{n}^{(1)}} \stackrel{n \rightarrow \infty}{\longrightarrow} i\left(H f_{1}\right)(p) \quad \lambda \text {-a.e. }
$$

If the subsequences $\left(t_{n}^{(1)}\right)_{n \in \mathbb{N}}, \ldots,\left(t_{n}^{(k-1)}\right)_{n \in \mathbb{N}}$ have been chosen, then the limit in Eq. (11.2 holds for $\left(t_{n}^{(k-1)}\right)_{n \in \mathbb{N}}$ and $f_{k}$. Hence there exists a subsequence $\left(t_{n}^{(k)}\right)_{n \in \mathbb{N}}$ of $\left(t_{n}^{(k-1)}\right)_{n \in \mathbb{N}}$ such that Eq. (11.3) holds for $\left(t_{n}^{(k)}\right)_{n \in \mathbb{N}}$ and $f_{1}, \ldots, f_{k}$. Because the countable union of null sets is a null set, there exists a null set $N$ such that for all $k \in \mathbb{N}$ we have

$$
\frac{v_{d_{n}}(p) f_{k}(p)-f_{k}(p)}{d_{n}} \stackrel{n \rightarrow \infty}{\longrightarrow} i\left(H f_{k}\right)(p) \quad \forall p \in \mathbb{R}^{d} \backslash N,
$$

where $\left(d_{n}\right)_{n \in \mathbb{N}}$ is the diagonal sequence given by $d_{n}:=t_{n}^{(n)}$.

Now put $h_{n}:=-i\left(v_{d_{n}}-I\right) d_{n}^{-1}, I$ being the identity matrix in $\mathbb{C}^{m \times m}$, and let $p \in \mathbb{R}^{d} \backslash N$. Suppose there is a matrix-valued function $F \in A^{m}$, i.e. the columns of $F$ are functions from $A$, such that $\operatorname{det}(F(p)) \neq 0$. Then

$$
\begin{aligned}
\left\|h_{m}(p)-h_{n}(p)\right\| \leq \| & \left(h_{m}(p) F(p)-(H F)(p)\right) F(p)^{-1} \| \\
& +\left\|\left(h_{n}(p) F(p)-(H F)(p)\right) F(p)^{-1}\right\| \stackrel{\min (m, n) \rightarrow \infty}{\longrightarrow} 0 .
\end{aligned}
$$

So in this case $\left(h_{n}(p)\right)_{n \in \mathbb{N}}$ converges for $n \rightarrow \infty$.

In order to see that $\left(h_{n}\right)_{n \in \mathbb{N}}$ converges $\lambda$-a.e., we show that

$$
M:=\left\{p \in \mathbb{R}^{d}: \operatorname{det}(F(p))=0 \quad \forall F \in A^{m}\right\}
$$

is a null set. Suppose $\lambda(M)>0$, then there exists a measurable set $S \subset M$ such that $0<\lambda(S)<\infty$. Consider the function $G: \mathbb{R}^{d} \rightarrow \mathbb{C}^{m \times m}, G(p):=\chi_{S}(p) I$. Since $G \in\left(L^{2}\right)^{m}$ and $A^{m}$ is dense in $\left(L^{2}\right)^{m}$, there is a sequence $\left(F_{n}\right)_{n \in \mathbb{N}}$ with $F_{n} \in A^{m}$ satifying $F_{n} \stackrel{n \rightarrow \infty}{\longrightarrow} G$. Thus there exists a null set $N_{0}$ and a subsequence $\left(F_{n_{k}}\right)_{k \in N}$ such that

$$
F_{n_{k}}(p) \stackrel{k \rightarrow \infty}{\longrightarrow} G(p) \quad \forall p \in \mathbb{R}^{d} \backslash N_{0} .
$$


But then for $p \in S \backslash N_{0}$ we have $0=\operatorname{det} F_{n_{k}}(p) \stackrel{k \rightarrow \infty}{\longrightarrow} \operatorname{det} G(p)=1$, which is impossible. Hence $M$ must be a null set, and $h_{n}$ converges $\lambda$-a.e. to a measurable matrix-valued function $h: \mathbb{R}^{d} \rightarrow \mathbb{C}^{m \times m}$, and for all $f \in A$ we have

$$
(H f)(p)=h(p) f(p) \quad \forall p \in \mathbb{R}^{d} \backslash(N \cup M) .
$$

Now for $g \in \mathscr{D}(H)$ let $\left(g_{n}\right)_{n \in \mathbb{N}}$ be a sequence in $A$ such that $\left(g_{n}, H g_{n}\right) \stackrel{n \rightarrow \infty}{\longrightarrow}$ $(g, H g)$. Then, by Riesz-Fischer, there exists a subsequence $\left(g_{a(n)}\right)_{n \in \mathbb{N}}$ of $\left(g_{n}\right)_{n \in \mathbb{N}}$ such that

$$
h(p) g_{a(n)}(p) \stackrel{n \rightarrow \infty}{\longrightarrow}(H g)(p) \quad \lambda \text {-a.e. }
$$

Because $g_{a(n)} \stackrel{n \rightarrow \infty}{\longrightarrow} g$ (again by Riesz-Fischer) there exists a subsequence $\left(g_{b(n)}\right)_{n \in \mathbb{N}}$ of $\left(g_{a(n)}\right)_{n \in \mathbb{N}}$ such that

$$
g_{b(n)}(p) \stackrel{n \rightarrow \infty}{\longrightarrow} g(p) \quad \lambda \text {-a.e. }
$$

hence

$$
(H g)(p)=\lim _{n \rightarrow \infty} h(p) g_{b(n)}(p)=h(p) g(p) \quad \lambda \text {-a.e. }
$$

By Lemma 11.3 we have $h=h^{*} \lambda$-a.e. and

$$
\mathscr{D}(H)=\left\{[f] \in L^{2}:[h f] \in L^{2}\right\} .
$$

Thus by changing $h$ on a set of measure zero we may consider $h(p)$ to be a self-adjoint matrix for all $p \in \mathbb{R}^{d}$.

Finally, Lemma 11.4 implies that $W(t)[f]:=\left[p \mapsto e^{i t h(p)} f(p)\right]$ is a continuous one-parameter unitary group on $L^{2}$. Let $K$ be the generator for $W$. The preceding arguments show that for some null sequence $\left(s_{n}\right)_{n \in \mathbb{N}}, k_{n}:=-i\left(e^{i s_{n} h}-I\right) / s_{n}$ converges pointwise $\lambda$-a.e. to a measurable matrix-valued function $k$ such that $\mathscr{D}(K)=$ $\left\{[f] \in L^{2}:[k f] \in L^{2}\right\}$ and $K[f]=[k f]$ for all $f \in \mathscr{D}(K)$. But on the other hand we have $k(p)=h(p) \lambda$-a.e., hence $K=H$, whence $V(t)=W(t)$. This completes the proof.

11.6 Lemma. Let $v: \mathbb{R} \rightarrow \mathbb{C}^{m \times m}$ be a matrix-valued function such that $v(0)=I$ and

$$
v(t) v(r)=v(t+r) \quad \forall t, r \in \mathbb{R} .
$$

If $v$ is continuous at some point $t_{0} \in \mathbb{R}$ then there exists a unique matrix $A \in \mathbb{C}^{m \times m}$ such that

$$
v(t)=e^{t A} \quad \forall t \in \mathbb{R} .
$$

Proof. Let $t \in \mathbb{R}$ and let $\left(t_{n}\right)_{n \in \mathbb{N}}$ be a sequence in $\mathbb{R}$ such that $t_{n} \stackrel{n \rightarrow \infty}{\longrightarrow} t$. Then

$$
v\left(t_{n}\right)=v\left(t_{n}+t_{0}-t\right) v\left(t-t_{0}\right) \stackrel{n \rightarrow \infty}{\longrightarrow} v\left(t_{0}\right) v\left(t-t_{0}\right)=v(t),
$$

so $v$ is continuous. Let $F: \mathbb{R} \rightarrow \mathbb{C}^{m \times m}$ be given by

$$
F(t):=\int_{0}^{t} v(r) d r
$$


Since $F(0)=0, F^{\prime}(t)=v(t)$ and $F^{\prime}(0)=I$, we have $t^{-1} F(t)-I \stackrel{t \rightarrow 0}{\longrightarrow} 0$. Hence there exists an $s>0$ such that $\left\|s^{-1} F(s)-I\right\|<1$. In particular, $F(s)$ is invertible. Then for $t \in \mathbb{R}$ we have

$$
v(t) F(s)=\int_{0}^{s} v(t) v(r) d r=\int_{0}^{s} v(t+r) d r=\int_{t}^{t+s} v(r) d r=F(t+s)-F(t),
$$

thus

$$
v(t)=(F(t+s)-F(t)) F(s)^{-1} \quad \forall t \in \mathbb{R} .
$$

This implies that $v$ is differentiable. Since $v^{\prime}(t)=v(t) A$, where $A:=(v(s)-I) F(s)^{-1}$, we have $\left(v(t) e^{-t A}\right)^{\prime}=v(t) A e^{-t A}-v(t) A e^{-t A}=0$. Hence $v(t) e^{-t A}=v(0) e^{-0 A}=I$, whence $v(t)=e^{t A}$ for all $t \in \mathbb{R}$. From $v^{\prime}(0)=A$ we see that $A$ is unique.

11.7. The logarithm of an operator. Let $X$ be a complex Banach space. For a bounded linear operator $A$ on $X$ denote by $\sigma(A)$ the spectrum of $A$. If $\sigma(A) \subset \mathbb{C} \backslash \mathbb{R}_{\leq 0}$ then, since $\log$ is holomorphic on $\mathbb{C} \backslash \mathbb{R}_{\leq 0}, \log (A)$ can be defined by means of a Dunford integral [[Yos65] VIII. 7.]

$$
\log (A):=\frac{1}{2 \pi i} \int_{C} \log (\lambda)(\lambda-A)^{-1} d \lambda
$$

where $C$ is a rectifiable Jordan curve in $\mathbb{C} \backslash \mathbb{R}_{\leq 0}$ oriented in the positive sense surrounding $\sigma(A)$. We then have

$$
\exp (\log (A))=(\exp \circ \log )(A)=A
$$

see [[Yos65] VIII. 7. Corollary 2].

If $B$ is a bounded linear operator on $X$ such that $\sigma(B) \subset \mathbb{R}+i(-\pi, \pi)$, the Spectral Mapping Theorem [[Yos65] VIII. 7. Corollary 1] implies $\sigma(\exp (B))=\exp (\sigma(B)) \subset$ $\mathbb{C} \backslash \mathbb{R}_{\leq 0}$, hence

$$
\log (\exp (B))=(\log \circ \exp )(B)=B .
$$

Let $A$ be a bounded linear operator on $X$ such that $\|A-I\|<1$. Then for $\lambda \in \mathbb{C}$ with $|\lambda-1| \geq 1$ we have that $A-\lambda I$ is invertible. Indeed: since $\left\|(\lambda-1)^{-1}(A-I)\right\|<1$, $B:=I-(\lambda-1)^{-1}(A-I)$ is invertible, hence $(1-\lambda) B=A-\lambda I$ is invertible. Thus $\sigma(A) \subset U:=\{z \in \mathbb{C}:|z-1|<1\}$. By means of

$$
\log (z)=\sum_{k=1}^{\infty} \frac{(-1)^{k+1}}{k}(z-1)^{k} \quad \forall z \in U,
$$

and [[Yos65] VIII. 7. Theorem (N. Dunford)] it follows that

$$
\log (A)=\sum_{k=1}^{\infty} \frac{(-1)^{k+1}}{k}(A-I)^{k} .
$$

By the submultiplicativity of the operator norm it is easy to see that the series converges uniformly on compact subsets of $\mathcal{U}:=\left\{A \in \mathbb{C}^{m \times m}:\|A-I\|<1\right\}$, thus log is continuous on $\mathcal{U}$. 
If $B$ is a bounded linear operator on $X$ such that $\|B\|<\log (2)$, then $\left\|e^{B}-I\right\| \leq$ $e^{\|B\|}-1<1$, hence $\sigma\left(e^{B}\right) \subset U \subset \mathbb{R}+i(-\pi, \pi)$ and

$$
\log e^{B}=B
$$

11.8 Lemma. Let $v: \mathbb{C} \rightarrow \mathbb{C}^{m \times m}$ be an entire matrix-valued function. If $\|v(z)-I\|<$ 1 for all $z$ in some non-empty open set $U$, then $z \mapsto \log v(z)$ is a holomorphic matrixvalued function on $U$, and $v(z)=e^{\log v(z)}$ for all $z \in U$.

Proof. For $n \in \mathbb{N}$ define the holomorphic matrix-valued functions $A_{n}: U \rightarrow \mathbb{C}^{m \times m}$,

$$
A_{n}(z):=\sum_{k=1}^{n} \frac{(-1)^{k+1}}{k}(v(z)-I)^{k} .
$$

If $K$ is a compact subset of $U$, then there exists an $\alpha \in(0,1)$ such that $\|v(z)-I\| \leq \alpha$ for all $z \in K$. By the submultiplicativity of the operator norm we have $\left\|(v(z)-I)^{k}\right\| \leq$ $\|v(z)-I\|^{k} \leq \alpha^{k}$, hence

$$
\sup _{z \in K}\left\|A_{n}(z)-\log (v(z))\right\| \leq \sum_{k=n+1}^{\infty} \frac{\alpha^{k}}{k} \stackrel{n \rightarrow \infty}{\longrightarrow} 0
$$

whence $z \mapsto \log (v(z))$ is holomorphic on $U$ (see, e.g., [[Rud70] Theorem 10.27] and note that $\left|A_{i j}\right| \leq\|A\|$ for all $\left.A \in \mathbb{C}^{m \times m}\right)$. $e^{\log v(z)}$.

From $\|v(z)-I\|<1$ it follows $\sigma(v(z)) \subset \mathbb{C} \backslash \mathbb{R}_{\leq 0}$. So by 11.7 we have $v(z)=$

11.9 Lemma. Let $\left(y_{n}\right)_{n \in \mathbb{N}}$ be an unbounded sequence in $\mathbb{R}$. Then there exists a $t \in \mathbb{R}$ such that $\left(e^{i t y_{n}}\right)_{n \in \mathbb{N}}$ does not converge.

Proof. Assume the contrary. Then $f(t):=\lim _{n \rightarrow \infty} e^{i t y_{n}}$ defines a function on $\mathbb{R}$ with

$$
f(0)=1, \quad f(s+t)=f(s) f(t) \quad \forall s, t \in \mathbb{R} .
$$

By [ [SS11] Chapter 4 Theorem 1.3] - which states that the pointwise limit of continuous complex valued functions is continuous except on a set of first category - and Lemma 11.6 there exists a $y \in \mathbb{R}$ such that $f(t)=e^{i t y}$ for all $t \in \mathbb{R}$. By Lebesgue's Dominated Convergence Theorem it is

$$
0=\lim _{n \rightarrow \infty} \frac{1}{i y_{n}}\left(e^{i t y_{n}}-1\right)=\lim _{n \rightarrow \infty} \int_{0}^{t} e^{i \tau y_{n}} d \tau=\int_{0}^{t} e^{i \tau y} d \tau .
$$

But this implies $y \neq 0$ and

$$
0=\frac{1}{i y}\left(e^{i t y}-1\right) \quad \forall t \in \mathbb{R},
$$

which is impossible. 
11.10 Theorem. Suppose that $V: \mathbb{R} \rightarrow L\left(L^{2}\left(\mathbb{R}^{d}, \mathbb{C}^{m}\right)\right)$,

$$
V(t)[f]:=\left[w_{t}(|\cdot|) f\right]
$$

defines a continuous one-parameter unitary group on $L^{2}\left(\mathbb{R}^{d}, \mathbb{C}^{m}, \lambda\right)$, where for each $t \in \mathbb{R}, w_{t}: \mathbb{C} \rightarrow \mathbb{C}^{m \times m}$ is an entire matrix-valued function. Then there exists an entire matrix-valued function $h_{c}: \mathbb{C} \rightarrow \mathbb{C}^{m \times m}$ such that $h_{c}(\rho)$ is self-adjoint for all $\rho \in \mathbb{R}$ and

$$
w_{t}(z)=\exp \left(i t h_{c}(z)\right) \quad \forall z \in \mathbb{C}, t \in \mathbb{R} .
$$

Moreover, the generator $H$ for $V$ has the domain

$$
\mathscr{D}(H)=\left\{[f] \in L^{2}\left(\mathbb{R}^{d}, \mathbb{C}^{m}, \lambda\right):\left[h_{c}(|\cdot|) f\right] \in L^{2}\left(\mathbb{R}^{d}, \mathbb{C}^{m}, \lambda\right)\right\},
$$

and satisfies $H[f]=\left[h_{c}(|\cdot|) f\right]$ for every $[f]$ in $\mathscr{D}(H)$.

Proof. By Proposition 11.5 there exists a measurable self-adjoint matrix-valued function $h: \mathbb{R}^{d} \rightarrow \mathbb{C}^{m \times m}$ such that for every $t \in \mathbb{R}$,

$$
w_{t}(|p|)=\exp (i \operatorname{th}(p)) \quad \lambda \text {-a.e. }
$$

Moreover, the generator $H$ for $V$ has the domain

$$
\mathscr{D}(H)=\left\{[f] \in L^{2}:[h f] \in L^{2}\right\}
$$

and satisfies $H[f]=[h f]$ for every $[f]$ in $\mathscr{D}(H)$.

For $n \in \mathbb{N}$ put $h_{n}:=-i n\left(w_{1 / n} \circ \eta-I\right)$, where $\eta: \mathbb{R}^{d} \rightarrow \mathbb{R}, \eta(p):=|p|$ and $I$ is the identity matrix on $\mathbb{C}^{m \times m}$. For each $n$ there exists a null set $N_{n}$ such that

$$
w_{1 / n}(|p|)=\exp (i h(p) / n) \quad \forall p \in \mathbb{R}^{d} \backslash N_{n} .
$$

Since the countable union of null sets is a null set, there exists a null set $N$ such that

$$
w_{1 / n}(|p|)=\exp (i h(p) / n) \quad \forall p \in \mathbb{R}^{d} \backslash N, n \in \mathbb{N} .
$$

In particular, $h_{n}(p) \stackrel{n \rightarrow \infty}{\longrightarrow} h(p)$ for all $p \in \mathbb{R}^{d} \backslash N$. And for all $p, p^{\prime} \in \mathbb{R}^{d} \backslash N$ with $|p|=\left|p^{\prime}\right|$ we have

$$
h(p)=\lim _{n \rightarrow \infty} h_{n}(p)=\lim _{n \rightarrow \infty} h_{n}\left(p^{\prime}\right)=h\left(p^{\prime}\right) .
$$

Hence, there exists a self-adjoint matrix-valued function $k: \mathbb{R}_{\geq 0} \rightarrow \mathbb{C}^{m \times m}$ such that

$$
k(|p|)=h(p) \quad \forall p \in \mathbb{R}^{d} \backslash N .
$$

Let $\mu:=\lambda \circ \eta^{-1}$ be the image measure on $\mathbb{R}_{\geq 0}$. For a fixed $t \in \mathbb{R}$ let

$$
S:=\left\{\rho \geq 0:\left\|w_{t}(\rho)-e^{i t k(\rho)}\right\|>0\right\} .
$$


Since $\lambda$ is complete and

$$
\eta^{-1}(S) \subset\left(\eta^{-1}(S) \backslash N\right) \cup N=\left\{p \in \mathbb{R}^{d} \backslash N:\left\|w_{t}(|p|)-e^{i \operatorname{th}(p)}\right\|>0\right\} \cup N,
$$

we have $\mu(S)=0$. Thus for each fixed $t \in \mathbb{R}$,

$$
w_{t}(\rho)=\exp (i t k(\rho)) \quad \mu \text {-a.e. }
$$

For $t, r \in \mathbb{R}$ there exists a $\mu$-null set $S_{t, r}$ such that

$$
w_{t}(\rho) w_{r}(\rho)=w_{t+r}(\rho) \quad \forall \rho \in \mathbb{R}_{\geq 0} \backslash S_{t, r} .
$$

Let $\rho \in R:=\mathbb{R}_{\geq 0} \backslash S_{t, r}$ and assume there exists an open neighborhood $U(\rho)$ of $\rho$ in $\mathbb{R}_{\geq 0}$ such that $U(\rho) \cap R=\{\rho\}$. But then $0<\mu(U(\rho))=\mu(U(\rho) \cap R)=\mu(\{\rho\})=0$, which is impossible. Hence every point of $R$ is a limit point of $R$. The Identity Theorem for holomorphic functions, see, e.g., [[Rud70] Theorem 10.18 fol.], implies

$$
w_{t}(z) w_{r}(z)=w_{t+r}(z) \quad \forall z \in \mathbb{C} .
$$

Moreover, since $w_{0}(\rho)=I \mu$-a.e., we have $w_{0}(z)=I$ for all $z \in \mathbb{C}$.

Consider the set

$$
G:=\left\{z \in \mathbb{C}: \exists B(z) \in \mathbb{C}^{m \times m} \text { such that } w_{t}(z)=e^{t B(z)} \forall t \in \mathbb{R}\right\} .
$$

Note that if $z \in G$, then $B(z)$ is unique by Lemma 11.6. Thus the set $G$ defines a function $B: G \rightarrow \mathbb{C}^{m \times m}$ satisfying $w_{t}(z)=e^{t B(z)}$ for all $z \in G, t \in \mathbb{R}$.

Claim 1. We have $\mathbb{R}_{>0} \subset G$.

Let $\rho>0$. For $n \in \mathbb{N}$ let $R_{n}:=\{r \geq 0:|r-\rho|<1 / n\}$ and $f_{n}: \mathbb{R} \rightarrow \mathbb{C}^{m \times m}$,

$$
f_{n}(t):=\mu\left(R_{n}\right)^{-1} \int_{R_{n}} w_{t}(q) d \mu(q) .
$$

Let $t \in \mathbb{R}$ be fixed and let $\varepsilon>0$. Since $w_{t}$ is continuous, there is an $N \in \mathbb{N}$ such that

$$
\left\|w_{t}(q)-w_{t}(\rho)\right\| \leq \varepsilon \quad \forall q \in R_{N} .
$$

Then for all $n \geq N$ we have

$$
\begin{aligned}
\left\|f_{n}(t)-w_{t}(\rho)\right\| & =\| \mu\left(R_{n}\right)^{-1} \int_{R_{n}}\left(w_{t}(q)-w_{t}(\rho) d \mu(q) \|\right. \\
& \leq \mu\left(R_{n}\right)^{-1} \int_{R_{n}}\left\|w_{t}(q)-w_{t}(\rho)\right\| d \mu(q) \leq \varepsilon .
\end{aligned}
$$

Thus $f_{n}(t) \stackrel{n \rightarrow \infty}{\longrightarrow} w_{t}(\rho)$ for each fixed $t$.

On the other hand, since

$$
f_{n}(t)=\mu\left(R_{n}\right)^{-1} \int_{R_{n}} e^{i t k(q)} d \mu(q),
$$


we see that the $f_{n}$ are continuous, indeed: Consider $u(t, q):=\mu\left(R_{n}\right)^{-1} e^{i t k(q)} \chi_{R_{n}}(q)$. Let $t_{j} \rightarrow t$. Because $\left\|u\left(t_{j}, q\right)\right\| \leq C \chi_{R_{n}}(q)$ for some constant $C$, we can apply Lebesgue's Dominated Convergence Theorem and get

$$
f_{n}(t)=\int u(t, q) d \mu(q)=\int \lim _{j \rightarrow \infty} u\left(t_{j}, q\right) d \mu(q)=\lim _{j \rightarrow \infty} \int u\left(t_{j}, q\right) d \mu(q)=\lim _{j \rightarrow \infty} f_{n}\left(t_{j}\right) .
$$

If we apply [[SS11] Chapter 4 Theorem 1.3] - which states that the pointwise limit of continuous complex valued functions is continuous except on a set of first category - to the matrix entries of $f_{n}$, we see that $t \mapsto w_{t}(\rho)$ is continuous at some point $t_{0} \in \mathbb{R}$. Then Lemma 11.6 implies that there exists a unique matrix $B(\rho)$ such that $w_{t}(\rho)=e^{t B(\rho)}$ for all $t \in \mathbb{R}$. Hence $\rho \in G$.

Claim 2. $G$ is closed.

Let $z \in \bar{G}$ and let $\left(z_{n}\right)_{n \in \mathbb{N}}$ be a sequence in $G$ converging to $z$. Then $t \mapsto$ $f_{n}(t):=w_{t}\left(z_{n}\right)=e^{t B\left(z_{n}\right)}$ is a sequence of continuous functions. Since $z \mapsto w_{t}(z)$ is entire, we have $\lim _{n \rightarrow \infty} f_{n}(t)=w_{t}(z)$ for each $t \in \mathbb{R}$. Thus $t \mapsto w_{t}(z)$ is the pointwise limit of continuous functions, and by the same argument as in the previous claim, we see that $w_{t}(z)=e^{t B(z)}$ for all $t \in \mathbb{R}$, for a unique matrix $B(z)$. Hence $z \in G$.

Claim 3. (a) For $z_{0} \in G$ and $\varepsilon>0$ there exists an open neighborhood $U$ of $z_{0}$ such that

$$
\left\|B(z)-B\left(z_{0}\right)\right\|<\varepsilon \quad \forall z \in U \cap G .
$$

(b) If $z_{0} \in \mathbb{C}$ is a limit point of $G$, then there exists an open neighborhood $U$ of $z_{0}$ such that $U \subset G$ and $B$ is holomorphic on $U$.

(a) It suffices to show that for every converging sequence $\left(z_{n}\right)_{n \in \mathbb{N}}$ in $G$ with limit $z_{0}$ we have $B\left(z_{n}\right) \stackrel{n \rightarrow \infty}{\longrightarrow} B\left(z_{0}\right)$.

Let $\sigma(B)$ denote the spectrum of $B \in \mathbb{C}^{m \times m}$.

Case 1. Suppose

$$
\bigcup_{n \geq 0} \operatorname{Im} \sigma\left(B\left(z_{n}\right)\right)
$$

is bounded. Then there exists a $t>0$ such that

$$
t \bigcup_{n \geq 0} \operatorname{Im} \sigma\left(B\left(z_{n}\right)\right) \subset(-\pi, \pi) .
$$

Thus $\log e^{t B\left(z_{n}\right)}=t B\left(z_{n}\right)$ for all $n \geq 0$ (see 11.7). We may also assume that $t$ is small enough such that

$$
\left\|e^{t B\left(z_{0}\right)}-I\right\|<1 .
$$

Since $w_{t}\left(z_{0}\right)=e^{t B\left(z_{0}\right)}$ and $w_{t}$ is continuous, there is an open neighborhood $\tilde{U}$ of $z_{0}$ such that

$$
\left\|w_{t}(z)-I\right\|<1 \quad \forall z \in \tilde{U}
$$

Thus $z \mapsto \log w_{t}(z)$ is holomorphic on $\tilde{U}$. By the convergence of $\left(z_{n}\right)_{n \in \mathbb{N}}$ we have $z_{n} \in \tilde{U}$ for all $n$ greater than some integer. So for all $n$ large enough it is

$$
t B\left(z_{n}\right)=\log e^{t B\left(z_{n}\right)}=\log w_{t}\left(z_{n}\right) \stackrel{n \rightarrow \infty}{\longrightarrow} \log w_{t}\left(z_{0}\right)=t B\left(z_{0}\right) .
$$


Case 2. Suppose

$$
\bigcup_{n \geq 0} \operatorname{Im} \sigma\left(B\left(z_{n}\right)\right)
$$

is unbounded. Then there exist $\lambda_{n} \in \mathbb{C}$ and $u_{n} \in \mathbb{C}^{m}$ such that

$$
\left\|u_{n}\right\|=1, \quad B\left(z_{n}\right) u_{n}=\lambda_{n} u_{n} \quad \forall n \in \mathbb{N}
$$

and $\left(\operatorname{Im} \lambda_{n}\right)_{n \in \mathbb{N}}$ is unbounded. Since $\left\|u_{n}\right\|=1$, we may assume that $\left(u_{n}\right)_{n \in \mathbb{N}}$ converges, otherwise we consider an appropriate subsequence. Let $x_{n}:=\operatorname{Re} \lambda_{n}$, and $y_{n}:=\operatorname{Im} \lambda_{n}$. Then for each $t \in \mathbb{R}$ we have

$$
w_{t}\left(z_{n}\right) u_{n}=e^{t B\left(z_{n}\right)} u_{n}=e^{t \lambda_{n}} u_{n}=e^{t x_{n}} e^{i t y_{n}} u_{n} \quad \forall n \in \mathbb{N} .
$$

Thus

$$
e^{t x_{n}}=\left\|e^{t x_{n}} e^{i t y_{n}} u_{n}\right\|=\left\|w_{t}\left(z_{n}\right) u_{n}\right\| \quad \forall n \in \mathbb{N} .
$$

Since the right-hand side converges, $\left(e^{t x_{n}}\right)_{n \in \mathbb{N}}$ converges too, and so does $\left(e^{-t x_{n}}\right)_{n \in \mathbb{N}}$. But then the right-hand side of

$$
e^{i t y_{n}}=e^{-t x_{n}}\left\langle u_{n}, e^{t x_{n}} e^{i t y_{n}} u_{n}\right\rangle=e^{-t x_{n}}\left\langle u_{n}, w_{t}\left(z_{n}\right) u_{n}\right\rangle
$$

also converges, and therefore $\left(e^{i t y_{n}}\right)_{n \in \mathbb{N}}$ converges for all $t \in \mathbb{R}$, which is impossible by Lemma 11.9.

(b) Let $z_{0}$ be a limit point of $G$. Since $G$ is closed, $z_{0} \in G$, thus $w_{t}\left(z_{0}\right)=e^{t B\left(z_{0}\right)}$ for all $t \in \mathbb{R}$. Hence, there exists an $r>0$ such that $\left\|w_{r}\left(z_{0}\right)-I\right\|<1$ and $\left\|r B\left(z_{0}\right)\right\|<$ $\log (2)$. By the continuity of $w_{r}$ and by (a) there exists an open connected neighborhood $U \subset \mathbb{C}$ of $z_{0}$ such that

$$
\left\|w_{r}(z)-I\right\|<1 \quad \forall z \in U
$$

and

$$
\|r B(z)\|<\log (2) \quad \forall z \in U \cap G .
$$

Put $A: U \rightarrow \mathbb{C}^{m \times m}$

$$
A(z):=\frac{\log w_{r}(z)}{r}
$$

and for $t \in \mathbb{R}$ define $f_{t}: U \rightarrow \mathbb{C}^{m \times m}$,

$$
f_{t}(z):=e^{t A(z)}
$$

By Lemma $11.8 A$ is holomorphic, thus $f_{t}$ is holomorphic. Moreover, we have

$$
B(z)=\frac{\log w_{r}(z)}{r}=A(z) \quad \forall z \in U \cap G .
$$

Hence $f_{t}(z)=e^{t A(z)}=e^{t B(z)}=w_{t}(z)$ for all $z \in U \cap G$. Since $z_{0}$ is a limit point of $U \cap G$, the Identity Theorem for holomorphic functions implies that $f_{t}(z)=w_{t}(z)$ for all $z \in U$. Hence $U \subset G$. Since $B(z)=A(z)$ for all $z \in U, B$ is holomorphic on $U$. 
Now we can show that $G=\mathbb{C}$. Since $\mathbb{R}_{\geq 0} \subset G$, claim 3 implies that $G$ contains a non-empty open set. Thus the interior of $G$, denoted as $G^{\circ}$, is non-empty. If $z$ is a limit point of $G^{\circ}$, then, because $G$ is closed, it follows by claim 3 that $z \in G^{\circ}$. So $G^{\circ}$ is closed. Since $\mathbb{C}$ is connected we must have $G^{\circ}=\mathbb{C}$, hence $G=\mathbb{C}$.

Finally, if we define $h_{c}: \mathbb{C} \rightarrow \mathbb{C}^{m \times m}, h_{c}(z):=-i B(z)$ we have $w_{t}(z)=e^{i t h_{c}(z)}$ for all $z \in \mathbb{C}$. Claim 3 implies that $h_{c}$ is holomorphic. Thus $g(z):=h_{c}(z)-h_{c}(\bar{z})^{*}$ is also holomorphic. Since $\left.w_{t}\right|_{\mathbb{R}_{\geq 0}}$ is unitary $\mu$-a.e., $\left.g\right|_{\mathbb{R}_{\geq 0}}=0 \mu$-a.e. and by continuity $\left.g\right|_{\mathbb{R}_{>0}}=0$. The Identity Theorem for holomorphic functions then implies $h_{c}(z)=h_{c}(\bar{z})^{*}$ for all $z \in \mathbb{C}$, thus $h_{c}(\rho)$ is self-adjoint for all $\rho \in \mathbb{R}$. 


\section{Growth Conditions on $e^{i t h}$ and the Linearity of $h$}

We show that if $e^{i t h}$, where $t \in \mathbb{R}$ and $h$ is a self-adjoint entire matrix-valued function, satisfies certain growth conditions, then $h$ must be a linear function. This is a result inspired by [[Cas84] Lemma 7 fol.].

12.1 Definition. For an exponentially bounded entire function $f: \mathbb{C}^{d} \rightarrow \mathbb{C}^{m \times m}$ let

$$
\delta(f):=\inf \left\{R \geq 0: \exists C \geq 0 \text { such that }\|f(z)\| \leq C e^{R|z|} \quad \forall z \in \mathbb{C}^{d}\right\} .
$$

12.2 Discussion. If $f: \mathbb{C}^{d} \rightarrow \mathbb{C}$ is an exponentially bounded entire function and $\left.f\right|_{\mathbb{R}^{d}} \in L^{2}$, then Corollary 10.10 implies that

$$
|f(z)| \leq C e^{\delta(f)|\operatorname{Im} z|} \quad \forall z \in \mathbb{C}^{d},
$$

for some constant $C>0$. The following Lemma shows a similar conclusion if $\left.f\right|_{\mathbb{R}^{d}}$ is bounded.

12.3 Lemma. Let $f: \mathbb{C}^{d} \rightarrow \mathbb{C}^{m \times m}$ be an exponentially bounded entire function such that $\left.f\right|_{\mathbb{R}^{d}}$ is bounded. Then

$$
\|f(z)\| \leq\left\|\left.f\right|_{\mathbb{R}^{d}}\right\|_{\infty} e^{\delta(f)|\operatorname{Im} z|} \quad \forall z \in \mathbb{C}^{d} .
$$

Proof. Put $\delta:=\delta(f)$ and let $\varepsilon>0$. Then Corollary 10.17 implies

$$
\|f(z)\| \leq\left\|\left.f\right|_{\mathbb{R}^{d}}\right\|_{\infty} e^{(\delta+\varepsilon)|\operatorname{Im} z|} \quad \forall z \in \mathbb{C}^{d} .
$$

Now $\varepsilon \rightarrow 0$ completes the proof.

12.4 Lemma. Let $f, g, h: \mathbb{C} \rightarrow \mathbb{C}$ be entire functions such that $f=g h \neq 0$. If $f$ and $g$ are exponentially bounded, then so is $h$. More precisely, if

$$
\max (|f(z)|,|g(z)|) \leq C e^{\tau|z|} \quad \forall z \in \mathbb{C},
$$

for some constants $C, \tau>0$, then

$$
|h(z)| \leq C^{\prime} e^{12 \tau|z|} \quad \forall z \in \mathbb{C},
$$

for some constant $C^{\prime}$.

Proof. (The proof makes use of Nevanlinna's Theory, for a brief introduction and detailed proofs see Appendix $\mathrm{H}$ and Lemma $\mathrm{H.4}$. Let $M(r, f):=\max _{|z|=r}|f(z)|$ and let

$$
\ln ^{+}(\alpha):=\max (0, \ln (\alpha)) \quad \text { for } \alpha>0 \text {. }
$$


Then for all $|z|=r>0$ we have, see [[Lev96] Lecture 2 (13) fol.],

$$
\ln M(r, h) \leq 3 \ln ^{+} M(2 r, f / C)+3 \ln ^{+} M(2 r, g / C)-3 \ln |c| \leq 12 \tau r-3 \ln |c|,
$$

for some constant $c \neq 0$. Hence, for all $|z|=r>0$ we have

$$
|h(z)| \leq M(r, h) \leq|c|^{-3} e^{12 \tau|z|} .
$$

By continuity the inequality also holds for $z=0$.

12.5 Proposition. Let $h: \mathbb{C} \rightarrow \mathbb{C}^{m \times m}$ be an entire matrix-valued function such that $h(x)$ is self-adjoint for all $x \in \mathbb{R}$. If

$$
\left\|e^{i t h(z)}\right\| \leq C e^{s|z|^{N}} \quad \forall z \in \mathbb{C},
$$

for some constants $C, t, s>0$ and $N \in \mathbb{N}$, then there exists a constant $k>0$ such that

$$
\|h(x)\| \leq k\left(1+|x|^{N}\right) \quad \forall x \in \mathbb{R} .
$$

Moreover, if $\delta(h)=0$, then $h$ is a polynomial of degree $N$ at most.

Proof. For $z \in \mathbb{C}$ let $\mu(z)$ be an eigenvalue of $h(z)$. Then $e^{i t \mu(z)}$ is an eigenvalue of $e^{i t h(z)}$ and

$$
\left|e^{i t \mu(z)}\right| \leq\left\|e^{i t h(z)}\right\| \leq C e^{s|z|^{N}} .
$$

So we obtain the estimate

$$
-\operatorname{Im} \mu(z) \leq \frac{\ln C}{t}+\frac{s}{t}|z|^{N}
$$

Since $h(x)^{*}=h(x)$ for all $x \in \mathbb{R}$, the entire function $z \mapsto h(\bar{z})^{*}-h(z)$ vanishes on $\mathbb{R}$. By the Identity Theorem for analytic functions we have $h(\bar{z})^{*}=h(z)$ for all $z \in \mathbb{C}$. This implies

$$
\left\|e^{-i t h(z)}\right\|=\left\|\left(e^{-i t h(z)}\right)^{*}\right\|=\left\|e^{i t h(z)^{*}}\right\|=\left\|e^{i t h(\bar{z})}\right\| \leq C e^{s|z|^{N}} \quad \forall z \in \mathbb{C} .
$$

Thus $\operatorname{Im} \mu(z) \leq \frac{\ln C}{t}+\frac{s}{t}|z|^{N}$, and we have

$$
|\operatorname{Im} \mu(z)| \leq c p(z),
$$

where $p(z):=1+|z|^{N}$ and $c>0$ is some constant independent of $\mu$. Let $u(z):=\operatorname{Re} \mu(z)$ and $v(z):=\operatorname{Im} \mu(z)$. Then

$$
\operatorname{Re}\left(-\mu^{2}\right)=\operatorname{Re}\left(v^{2}-u^{2}-2 i u v\right) \leq v^{2} \leq c^{2} p^{2}
$$

implies

$$
\operatorname{Re}\left(-\operatorname{tr}\left(h^{2}(z)\right)\right) \leq m c^{2} p^{2}(z) \quad \forall z \in \mathbb{C},
$$

and thus we find

$$
\left|e^{-\operatorname{tr}\left(h^{2}(z)\right)}\right|=e^{\operatorname{Re}\left(-\operatorname{tr}\left(h^{2}(z)\right)\right)} \leq e^{m c^{2} p^{2}(z)} .
$$


This shows that $e^{-\operatorname{tr}\left(h^{2}(\cdot)\right)}$ is an entire function of finite order, and by Hadamard's Factorization Theorem [[Con78] XI.3.4] $\operatorname{tr}\left(h^{2}(\cdot)\right)$ must be a polynomial of degree $2 \mathrm{~N}$ at most. For $x \in \mathbb{R}$ the matrix $h(x)$ is self-adjoint, therefore $\|h(x)\|=\max |\sigma(h(x))|$, where $\sigma(h(x))$ denotes the spectrum of $h(x)$. Since the eigenvalues for self-adjoint matrices are real, we have

$$
\|h(x)\|^{2}=\max |\sigma(h(x))|^{2}=\max \sigma(h(x))^{2}=\max \sigma\left(h^{2}(x)\right) \leq \operatorname{tr} h^{2}(x) \quad \forall x \in \mathbb{R} .
$$

Thus there exists a constant $k>0$ such that

$$
\|h(x)\| \leq k p(x) \quad \forall x \in \mathbb{R} .
$$

Let $\delta(h)=0$ and $1 \leq a, b \leq m$. Put $\xi_{ \pm}(z):=h_{a b}(z) /( \pm i+z)^{N}$. Then for every $\varepsilon>0$ there exists a $C_{\varepsilon}>0$ such that

$$
\left|\xi_{ \pm}(z)\right| \leq C_{\varepsilon} e^{\varepsilon|z|} \quad \forall z \in G_{ \pm}:=\{w \in \mathbb{C}: \pm \operatorname{Im} w \geq 0\} .
$$

Since

$$
\left|\xi_{ \pm}(x)\right|^{2} \leq k^{2} \frac{\left(1+x^{N}\right)^{2}}{\left(1+x^{2}\right)^{N}} \leq 2 k^{2} \quad \forall x \in \mathbb{R}
$$

a Corollary of the Phragmén-Lindelöf Theorem (see [[Con78] Corollary VI.4.4 p. 140]) implies that $\xi_{ \pm}$is bounded in $G_{ \pm}$. Hence there exists a constant $k^{\prime}>0$ such that $\left|h_{a b}(z)\right| \leq k^{\prime} p(z)$ for all $z \in \mathbb{C}$. By Liouville's Theorem $h_{a b}(z)$ is a polynomial of degree $N$ at most.

The following Theorem is inspired by [[Cas84] Lemma 7 fol.].

12.6 Theorem. Let $T>0$ and let $h: \mathbb{C} \rightarrow \mathbb{C}^{m \times m}$ be an entire matrix-valued function such that

(a) $h(\rho)$ is self-adjoint for all $\rho \in \mathbb{R}$.

(b) $e^{i t h(\cdot)}$ is exponentially bounded for every $t \in[0, T]$.

(c) There exists a function $f:[0, T] \rightarrow \mathbb{R}$ such that $f(0)=0, f$ is continuous at 0 and $\delta\left(e^{i t h(\cdot)}\right) \leq f(t)$ for all $t \in[0, T]$.

Then $\delta(h)=0$ and there are self-adjoint matrices $A, B \in \mathbb{C}^{m \times m}$ such that $h(z)=$ $A+B z$ for all $z \in \mathbb{C}$.

Proof. In view of Proposition 12.5 we only need to show that $\delta(h)=0$.

By continuity there exists a $t_{0} \in(0, T]$ such that $f(t)<1$ for all $t \in\left[0, t_{0}\right]$. Put $g:\left[0, t_{0}\right] \rightarrow \mathbb{R}, g(t):=\sup _{\tau \in[0, t]} f(\tau)$. Then $f(t) \leq g(t)$ for all $t \in\left[0, t_{0}\right], g$ is monotonically increasing, $g(0)=0$ and $g$ is continuous at 0 .

For $t \in\left[0, t_{0}\right]$ define $W_{t}: \mathbb{C} \rightarrow \mathbb{C}^{m \times m}$,

$$
W_{t}(z):=\int_{0}^{t} e^{i \tau h(z)} d \tau .
$$


If $\left(z_{n}\right)_{n \in \mathbb{N}}$ is a converging sequence in $\mathbb{C}$ with limit $z$, then there exists an $R \geq 0$ such that $\left\|h\left(z_{n}\right)\right\| \leq R$ for all $n \in \mathbb{N}$, hence $\left\|e^{i \tau h\left(z_{n}\right)}\right\| \leq e^{\tau R}$ for all $n \in \mathbb{N}, \tau \in\left[0, t_{0}\right]$. Since $e^{(\cdot) R}$ is an integrable dominating function, Lebesgue's Dominated Convergence Theorem implies that $W_{t}\left(z_{n}\right) \stackrel{n \rightarrow \infty}{\longrightarrow} W_{t}(z)$, whence $W_{t}$ is continuous. Using the power series of $e^{i t h(z)}$ we find

$$
i h(z) W_{t}(z)=e^{i t h(z)}-I \quad \forall z \in \mathbb{C} \quad \forall t \in\left[0, t_{0}\right] .
$$

Let $\tilde{h}(z)$ denote the adjugate 5 matrix of $h(z)$. Using the property $A \tilde{A}=\tilde{A} A=\operatorname{det}(A) I$ we obtain

$$
i \operatorname{det}(h(z)) W_{t}(z)=\tilde{h}(z)\left(e^{i t h(z)}-I\right) .
$$

Note that $\operatorname{det}(h(\cdot)), \tilde{h}$ and $e^{i t h(\cdot)}$ are entire functions. This shows that the matrix elements of $W_{t}$ are meromorphic functions, and since $W_{t}$ is continuous, these functions must be entire. Hence $W_{t}$ is entire. Because $h(x)$ is self-adjoint, we have $\left\|e^{i \tau h(x)}\right\|=1$ for all $x \in \mathbb{R}$. By the assumption $\delta\left(e^{i \tau h(\cdot)}\right) \leq f(\tau)$ it follows from Lemma 12.3 that

$$
\left\|e^{i \tau h(z)}\right\| \leq e^{f(\tau)|\operatorname{Im} z|} \quad \forall z \in \mathbb{C} \quad \forall \tau \in[0, T] .
$$

Let $t \in\left[0, t_{0}\right]$. Since $f(\tau) \leq g(t)$ for all $\tau \in[0, t]$, we have

$$
\left\|W_{t}(z)\right\| \leq \int_{0}^{t} e^{g(t)|\operatorname{Im} z|} d \tau=t e^{g(t)|\operatorname{Im} z|} \quad \forall z \in \mathbb{C} .
$$

If $\tilde{W}_{t}(z)$ is the adjugate matrix of $W_{t}(z)$, then

$$
i h(z) \operatorname{det}\left(W_{t}(z)\right)=\left(e^{i t h(z)}-I\right) \tilde{W}_{t}(z) \quad \forall z \in \mathbb{C} \quad \forall t \in\left[0, t_{0}\right] .
$$

Let $i, j \in\{1, \ldots, m\}$. Since the determinant of an $n \times n$ matrix has $n$ ! terms, and each term contains $n$ products, we have

$$
\left|\tilde{W}_{t}(z)_{i j}\right| \leq(m-1) ! t^{m-1} e^{(m-1) g(t)|\operatorname{Im} z|} \quad \forall z \in \mathbb{C} \quad \forall t \in\left[0, t_{0}\right] .
$$

This estimate and $\left|\left(e^{i t h(z)}-I\right)_{i j}\right| \leq 2 e^{g(t)|\operatorname{Im} z|}$ gives

$$
\left|h(z)_{i j} \operatorname{det}\left(W_{t}(z)\right)\right| \leq \sum_{n=1}^{m}\left|\left(e^{i t h(z)}-I\right)_{i n}\right|\left|\tilde{W}_{t}(z)_{n j}\right| \leq 2 m ! t^{m-1} e^{m g(t)|\operatorname{Im} z|}
$$

for all $z \in \mathbb{C}, t \in\left[0, t_{0}\right]$.

In order to apply Lemma 12.4 we show that $\operatorname{det}\left(W_{t}(\cdot)\right) \neq 0$ for sufficiently small $t>0$. Put $F: \mathbb{R}_{\geq 0} \rightarrow \mathbb{C}^{m \times m}, F(t):=W_{t}(0)$. Then $F(0)=0$ and $F^{\prime}(0)=I$ implies

$$
\frac{F(t)}{t}=\frac{F(t)-F(0)}{t-0} \stackrel{t \rightarrow 0}{\longrightarrow} I \text {. }
$$

\footnotetext{
${ }^{5}$ The adjugate $\tilde{A}$ of a matrix $A$ is given by the matrix elements $\tilde{A}_{i j}:=(-1)^{i+j} \operatorname{det}\left(A^{(j, i)}\right)$, where $A^{(i, j)}$ denotes the $(m-1) \times(m-1)$ matrix obtained by removing the $i$-th row and $j$-th column of $A$.
} 
Thus there exists an $s \in\left(0, t_{0}\right]$ such that

$$
\left\|t^{-1} W_{t}(0)-I\right\|<1 \quad \forall t \in(0, s) .
$$

Hence $\operatorname{det} W_{t}(0) \neq 0$ for all $t \in(0, s)$, whence $\operatorname{det} W_{t}(\cdot) \neq 0$ for all $t \in(0, s)$. Suppose $h_{i j} \neq 0$. Then $h(\cdot)_{i j} \operatorname{det}\left(W_{t}(\cdot)\right) \neq 0$ for all $t \in(0, s)$. Since

$$
\left|\operatorname{det}\left(W_{t}(z)\right)\right| \leq m ! t^{m} e^{m g(t)|\operatorname{Im} z|},
$$

Lemma 12.4 implies that for each $t \in(0, s)$ there exists a $C_{t}>0$ such that

$$
\left|h(z)_{i j}\right| \leq C_{t} e^{12 m g(t)|z|} \quad \forall z \in \mathbb{C}
$$

If $h_{i j}=0$ then this estimate holds trivially. Because $\|h(z)\| \leq \kappa \sum_{i, j=1}^{m}\left|h(z)_{i j}\right|$ for some constant $\kappa>0$ we have $\delta(h) \leq 12 m g(t)$ for all $t \in(0, s)$. Now $t \rightarrow 0$ completes the proof.

12.7. Remark and Examples. In the one dimensional case, i.e. $m=1$, Hadamard's Factorization Theorem shows that the condition $\delta\left(e^{i t h(\cdot)}\right)<\infty$ for some $t>0$ already implies that $h$ is a polynomial of degree 1 at most. Considering $h(z):=\left(\begin{array}{cc}0 & e^{i z} \\ e^{-i z} & 0\end{array}\right)$ we see that this is not the case for $m>1$. Here we have $\left\|e^{i t h(z)}\right\| \leq 2 e^{|\operatorname{Im} z|}$, thus $\delta\left(e^{i t h(\cdot)}\right) \leq 1$ for all $t \geq 0$, but $\delta(h)=1$.

The example $h(z):=\left(\begin{array}{cc}0 & z^{2} \\ 0 & 0\end{array}\right)$ shows that the self-adjointness of $h(x)$ for $x \in \mathbb{R}$ is crucial for Proposition 12.5, we have $\delta\left(e^{i t h(\cdot)}\right)=0$ for all $t \geq 0$ and $\delta(h)=0$, but $h$ is not a polynomial of degree 1 . 


\section{Appendices}

\section{A Projection- and Positive Operator-Valued Measures}

A.1 Definition. Let $(\Omega, \mathscr{A})$ be a measurable space and let $\mathscr{H}$ be a complex Hilbert space. We say $E: \mathscr{A} \rightarrow L(\mathscr{H})$ is a projection-valued measure (PVM) if

(a) $E(A)$ is an orthogonal projection for all $A \in \mathscr{A}$.

(b) $E(\Omega)=I$, where $I$ denotes the identity operator on $\mathscr{H}$.

(c) For any sequence $\left(A_{n}\right)_{n \in \mathbb{N}}$ of mutually disjoint $A_{n} \in \mathscr{A}$ we have

$$
E\left(\bigcup_{n \in \mathbb{N}} A_{n}\right) \psi=\sum_{n \in \mathbb{N}} E\left(A_{n}\right) \psi \quad \forall \psi \in \mathscr{H} .
$$

If $E$ satisfies

(a') $0 \leq E(A)$ for all $A \in \mathscr{A}$,

instead of (a), then $E$ is called a positive operator-valued measure (POVM). Since every self-adjoint projection is positive, it is clear that every PVM is a POVM.

The tuple $(\mathscr{A}, E)$ will be called complete if $N \in \mathscr{A}$ and $E(N)=0$ implies $S \in \mathscr{A}$ for every $S \subset N$.

A.2 Lemma. Let $(\Omega, \mathscr{A})$ be a measurable space, let $\mathscr{H}$ be a complex Hilbert space and let $E: \mathscr{A} \rightarrow L(\mathscr{H})$ be a PVM or a POVM. Then for all $A, B \in \mathscr{A}$ we have

(a) $E\left(A^{c}\right)=I-E(A)$.

(b) $E(\varnothing)=0$.

(c) $A \subset B$ implies $E(A) \leq E(B)$.

(d) $E(A) \leq I$.

(e) $E(A \cup B)=E(A)+E(B)-E(A \cap B)$.

Proof. (a) follows from $I=E(\Omega)=E(A)+E\left(A^{c}\right)$. (b) follows from (a). Let $A \subset B$. Then

$$
E(B)=E(A \cup(B \backslash A))=E(A)+E(B \backslash A)
$$

implies (c). (d) follows from (c), since $E(A) \leq E(\Omega)=I$. We have

$$
\begin{aligned}
E(A \cup B) & =E(A)+E(B \backslash A)=E(A)+E(B \backslash A)+E(A \cap B)-E(A \cap B) \\
& =E(A)+E(B)-E(A \cap B) .
\end{aligned}
$$

This proves (e). 
A.3 Lemma. Let $P$ and $Q$ be projections (not necessarily orthogonal projections). If $P Q+Q P=0$ then $P Q=0$.

Proof. We have $P Q=P P Q Q=-P Q P Q=P Q Q P=P Q P=-P P Q=-P Q$, hence $P Q=0$.

A.4 Lemma. Let $(\Omega, \mathscr{A})$ be a measurable space, let $\mathscr{H}$ be a complex Hilbert space and let $E: \mathscr{A} \rightarrow L(\mathscr{H})$ be a projection-valued measure. Then

$$
E(A \cap B)=E(A) E(B) \quad \forall A, B \in \mathscr{A} .
$$

Proof. For $A \in \mathscr{A}$ we have $E(A) E\left(A^{c}\right)=E(A)(I-E(A))=0$. If $A$ and $B$ are disjoint we have

$$
\begin{aligned}
E(A \cup B) & =(E(A)+E(B))^{2}=E(A)+E(A) E(B)+E(B) E(A)+E(B) \\
& =E(A \cup B)+E(A) E(B)+E(B) E(A) .
\end{aligned}
$$

Thus $E(A) E(B)+E(B) E(A)=0$ and from Lemma A.3 we have $E(A) E(B)=0$. For arbitrary $A, B \in \mathscr{A}$ we have

$$
\begin{aligned}
E(A) E(B) & =(E(A \backslash(A \cap B))+E(A \cap B))(E(B \backslash(A \cap B))+E(A \cap B)) \\
& =E(A \cap B) E(A \cap B)=E(A \cap B) .
\end{aligned}
$$

A.5 Remark. Note that for the proof of Lemma A.4 the projections $E(A)$ for $A \in \mathscr{A}$ need not be orthogonal.

A.6 Lemma. Let $\mathscr{H}$ be a complex Hilbert space, and let $A, B \in L(\mathscr{H})$. If $0 \leq A \leq$ $B \leq A$ then $A=B$.

Proof. Put $C:=B-A$. Then we have $0 \leq\langle\psi, C \psi\rangle \leq 0$ for all $\psi \in \mathscr{H}$, hence $C=0$, see, e.g., [[Rud91] Theorem 12.7 p. 310], or use $0=\langle\psi, C \psi\rangle=\|\sqrt{C} \psi\|^{2}$.

A.7 Definition. Let $(\Omega, \mathscr{A})$ be a measurable space, let $\mathscr{H}$ be a complex Hilbert space and let $E: \mathscr{A} \rightarrow L(\mathscr{H})$ be a PVM or a POVM. Then $(\mathscr{A}, E)$ is called complete, if $S \subset N \in \mathscr{A}$ and $E(N)=0$ implies $S \in \mathscr{A}$.

A.8. The completion of a PVM (or a POVM). Let $(\Omega, \mathscr{A}$ ) be a measurable space, let $\mathscr{H}$ be a complex Hilbert space and let $E: \mathscr{A} \rightarrow L(\mathscr{H})$ be a PVM (or a POVM).

Similar to the completion of a measure space [[HS65] Theorem (11.21) p. 155] we consider the following completion of $\mathscr{A}$ and $E$. Let

$$
\mathscr{N}:=\{S \subset \Omega: \exists N \in \mathscr{A}: S \subset N, E(N)=0\} .
$$

Define

$$
\mathscr{A}_{c}:=\{A \cup S: A \in \mathscr{A}, S \in \mathscr{N}\},
$$

and put

$$
E_{c}(A \cup S):=E(A),
$$


for $A \in \mathscr{A}$ and $S \in \mathscr{N}$.

By the following statements it is justified to call $\left(\mathscr{A}_{c}, E_{c}\right)$ the completion of $(\mathscr{A}, E)$.

(a) $\mathscr{A}_{c}$ is a $\sigma$-algebra.

(b) $E_{c}$ is a PVM (or a POVM).

(c) $\left(\mathscr{A}_{c}, E_{c}\right)$ is complete, $\mathscr{A} \subset \mathscr{A}_{c}$ and $\left.E_{c}\right|_{\mathscr{A}}=E$.

(d) If $\left(\mathscr{A}^{\prime}, E^{\prime}\right)$ is complete such that $\mathscr{A} \subset \mathscr{A}^{\prime}$ and $\left.E^{\prime}\right|_{\mathscr{A}}=E$ then $\mathscr{A}_{c} \subset \mathscr{A}^{\prime}$ and $\left.E^{\prime}\right|_{\mathscr{A}_{c}}=E_{c}$.

Proof. (a) Obviously, $\Omega \in \mathscr{A}_{c}$. Let $A \in \mathscr{A}, S \in \mathscr{N}$ and $N \in \mathscr{A}$ such that $S \subset N$ and $E(N)=0$. Then $(A \cup S)^{c}=\left(A^{c} \cap N^{c}\right) \cup R$, where $R:=N \cap(A \cup S)^{c}$, shows that $(A \cup S)^{c} \in \mathscr{A}_{c}$. Let $A_{1}^{\prime}, A_{2}^{\prime}, \ldots \in \mathscr{A}_{c}$. Then for each $k \in \mathbb{N}$ let $A_{k}^{\prime}=A_{k} \cup S_{k}$, where $A_{k} \in \mathscr{A}$ and $S_{k} \in \mathscr{N}$. Let $N_{k} \in \mathscr{A}$ such that $S_{k} \subset N_{k}$ and $E\left(N_{k}\right)=0$ for all $k \in \mathbb{N}$. Put

$$
A:=\bigcup_{k \in N} A_{k}, \quad S:=\bigcup_{k \in N} S_{k} \subset N:=\bigcup_{k \in N} N_{k} .
$$

Let $M_{1}:=N_{1}$ and put $M_{k}:=N_{k} \backslash\left(N_{1} \cup \ldots \cup N_{k-1}\right)$ for $k>1$. Then $M_{k} \cap M_{j}=\varnothing$ for $k \neq j$ and $N=\bigcup_{k \in \mathbb{N}} M_{k}$. Thus for every $\psi \in \mathscr{H}$ we have $E(N) \psi=E\left(\bigcup_{k \in \mathbb{N}} M_{k}\right) \psi=$ $\lim _{n \rightarrow \infty} \sum_{k=1}^{n} E\left(M_{k}\right) \psi=0$, since $M_{k} \subset N_{k}$ implies $E\left(M_{k}\right) \leq E\left(N_{k}\right)=0$, thus $E\left(M_{k}\right)=$ 0 for all $k$. Now $\bigcup_{k \in N} A_{k}^{\prime}=A \cup S$ and $E(N)=0$ implies that $\bigcup_{k \in N} A_{k}^{\prime} \in \mathscr{A}_{c}$.

(b) We show that $E_{c}$ is well-defined. Suppose $A_{1} \cup S_{1}=A_{2} \cup S_{2}$, where $A_{1}, A_{2} \in \mathscr{A}$ and $S_{1}, S_{2} \in \mathscr{N}$. We have to show that $E\left(A_{1}\right)=E\left(A_{2}\right)$. Let $S_{1} \subset N_{1}$ and $S_{2} \subset N_{2}$, where $N_{1}, N_{2} \in \mathscr{A}$ such that $E\left(N_{1}\right)=E\left(N_{2}\right)=0$. Then $A_{2} \subset A_{2} \cup S_{2}=A_{1} \cup S_{1} \subset$ $A_{1} \cup N_{1}$ implies

$$
E\left(A_{2}\right) \leq E\left(A_{1} \cup N_{1}\right)=E\left(A_{1} \cup\left(N_{1} \backslash A_{1}\right)\right)=E\left(A_{1}\right)+E\left(N_{1} \backslash A_{1}\right)=E\left(A_{1}\right) .
$$

By the same reasoning $E\left(A_{1}\right) \leq E\left(A_{2}\right)$. Hence $E\left(A_{1}\right)=E\left(A_{2}\right)$.

The PVM (or a POVM) properties: By definition $E_{c}(B)$ is a self-adjoint projection (or positive) for all $B \in \mathscr{A}_{c}$. Clearly $E_{c}(\Omega)=I$. Let $\left(B_{n}\right)_{n \in \mathbb{N}}$ be a sequence of mutually disjoint sets in $\mathscr{A}_{c}$. Let $B_{n}=A_{n} \cup S_{n}$, where $A_{n} \in \mathscr{A}$ and $S_{n} \in \mathscr{N}$, and let $N_{n} \in \mathscr{A}$ such that $S_{n} \subset N_{n}$ and $E\left(N_{n}\right)=0$. Then $A_{k} \cap A_{j}=\varnothing$ for all $k \neq j$. For $\psi \in \mathscr{H}$ we have

$$
\begin{aligned}
E_{c}\left(\bigcup_{n \in \mathbb{N}} B_{n}\right) \psi & =E_{c}\left(\left(\bigcup_{n \in \mathbb{N}} A_{n}\right) \cup\left(\bigcup_{n \in \mathbb{N}} S_{n}\right)\right) \psi=E\left(\bigcup_{n \in \mathbb{N}} A_{n}\right) \psi=\sum_{n \in \mathbb{N}} E\left(A_{n}\right) \psi \\
& =\sum_{n \in \mathbb{N}} E_{c}\left(A_{n} \cup S_{n}\right) \psi=\sum_{n \in \mathbb{N}} E_{c}\left(B_{n}\right) \psi,
\end{aligned}
$$

where we used $\bigcup_{k \in N} S_{k} \subset N:=\bigcup_{k \in N} N_{k}$ and $E(N)=0$, as shown in (a). 
(c) Let $E_{c}(M)=0$ for some $M \in \mathscr{A}_{c}$ and let $T \subset M$. Then there exist $A, N \in \mathscr{A}$, $S \in \mathscr{N}$ such that $M=A \cup S, S \subset N$ and $E(N)=0$. By definition $E_{c}(M)=E(A)=0$. Since $T \subset M=A \cup S \subset A \cup N$ and

$$
0 \leq E(A \cup N)=E(A)+E(N \backslash A) \leq E(A)+E(N)=0,
$$

we have $T \in \mathscr{N}$, hence $T \in \mathscr{A}_{c} . \mathscr{A} \subset \mathscr{A}_{c}$ and $\left.E_{c}\right|_{\mathscr{A}}=E$ are obviously true.

(d) Let $B \in \mathscr{A}_{c}$. Then we may write $B=A \cup S$, where $A, N \in \mathscr{A}, S \in \mathscr{N}$ such that $S \subset N$ and $E(N)=0$. Since $E^{\prime}(N)=E(N)=0$ and by the completeness of $\mathscr{A}^{\prime}$, we have $S \in \mathscr{A}^{\prime}$. Because $A \in \mathscr{A} \subset \mathscr{A}^{\prime}$, we have $B=A \cup S \in \mathscr{A}^{\prime}$. Moreover, $0 \leq E^{\prime}(S \backslash A) \leq E^{\prime}(N)=0$, therefore

$$
E^{\prime}(B)=E^{\prime}(A \cup S)=E^{\prime}(A)+E^{\prime}(S \backslash A)=E(A)=E_{c}(B) .
$$

This completes the proof. 


\section{B The Covering Groups for $L_{+}^{\uparrow}$ and $S O(3)$}

We summarize, loosely following [[Tha92 Sec. 2.5], some important facts about the $S O(3)$ and the proper orthochronous Lorentz group $L_{+}^{\uparrow}$ (also denoted as $S O^{+}(1,3)$ ) and their covering groups, the $S U(2)$ and the $S L(2, \mathbb{C})$.

The Pauli matrices $\sigma_{i}(i=1,2,3)$ and $\sigma_{0}$ are the self-adjoint $2 \times 2$ matrices given by

$$
\sigma_{0}:=\left(\begin{array}{cc}
1 & 0 \\
0 & 1
\end{array}\right), \quad \sigma_{1}:=\left(\begin{array}{cc}
0 & 1 \\
1 & 0
\end{array}\right), \quad \sigma_{2}:=\left(\begin{array}{cc}
0 & -i \\
i & 0
\end{array}\right), \quad \sigma_{3}:=\left(\begin{array}{cc}
1 & 0 \\
0 & -1
\end{array}\right) .
$$

They satisfy

$$
\left[\sigma_{a}, \sigma_{b}\right]=2 i \sum_{c=1}^{3} \varepsilon_{a b c} \sigma_{c}, \quad\left\{\sigma_{a}, \sigma_{b}\right\}=2 \delta_{a b} 1_{2} \quad(a, b=1,2,3),
$$

where $[A, B]:=A B-B A$ and $\{A, B\}:=A B+B A$. Both relations are equivalent to

$$
\sigma_{a} \sigma_{b}=\delta_{a b} 1_{2}+i \sum_{c=1}^{3} \varepsilon_{a b c} \sigma_{c} \quad(a, b=1,2,3) .
$$

The mapping $(M, N) \mapsto\langle M, N\rangle:=\frac{1}{2} \operatorname{tr}\left(M^{*} N\right)$ defines a scalar product on $\mathbb{C}^{2 \times 2}$ for which $\sigma_{\mu}(\mu=0,1,2,3)$ is an orthonormal base.

Define $\sigma: \mathbb{C}^{4} \rightarrow \mathbb{C}^{2 \times 2}$ by

$$
\sigma(x):=\sum_{\mu=0}^{3} x_{\mu} \sigma_{\mu}
$$

For $\mathbf{x} \in \mathbb{C}^{3}$ we put $\sigma(\mathbf{x}):=\sum_{i=1}^{3} x_{i} \sigma_{i}$. Since the $\sigma_{\mu}$ form a base, $\sigma$ is a bijection: For $N \in \mathbb{C}^{2 \times 2}$ and $y \in \mathbb{C}^{4}$ it is

$$
\sigma(y)=N \Longleftrightarrow y_{\lambda}=\left\langle\sigma_{\lambda}, N\right\rangle
$$

Thus

$$
\sigma(\Lambda(M) x)=M \sigma(x) M^{*} \quad\left(M^{*}:=\bar{M}^{T}\right)
$$

defines a mapping $\Lambda: \mathbb{C}^{2 \times 2} \rightarrow \mathbb{C}^{4 \times 4}$. In fact, $\Lambda$ is an algebra homomorphism, for if $M, N \in \mathbb{C}^{2 \times 2}$ we have

$$
\sigma(\Lambda(M N) x)=M N \sigma(x) N^{*} M^{*}=M \sigma(\Lambda(N) x) M^{*}=\sigma(\Lambda(M) \Lambda(N) x),
$$

hence $\Lambda(M N)=\Lambda(M) \Lambda(N)$.

It is useful to know that

$$
\Lambda(M)_{\mu \nu}=\left\langle\sigma_{\mu}, M \sigma_{\nu} M^{*}\right\rangle=\frac{1}{2} \operatorname{tr}\left(\sigma_{\mu} M \sigma_{\nu} M^{*}\right)
$$


and

$$
\Lambda\left(M^{*}\right)=\Lambda(M)^{T}, \quad M \sigma_{\mu} M^{*}=\left(\Lambda\left(M^{*}\right) \sigma\right)_{\mu}:=\sum_{\nu=0}^{3} \Lambda\left(M^{*}\right)_{\mu \nu} \sigma_{\nu}, \quad \Lambda(M) \in \mathbb{R}^{4 \times 4},
$$

for all $M \in \mathbb{C}^{2 \times 2}$.

The fact that $\operatorname{det}(\sigma(x))=x_{0}^{2}-x_{1}^{2}-x_{2}^{2}-x_{3}^{2}$ for all $x \in \mathbb{R}^{4}$ leads to the following Theorem, which shows that the restrictions of $\Lambda$ to $S U(2)$ and $S L(2, \mathbb{C})$ are the covering homomorphisms for the associated groups.

B.1 Theorem. The homomorphism $\Lambda$ maps $S L(2, \mathbb{C})$ onto the proper orthochronous Lorentz group $L_{+}^{\uparrow}$ and $S U(2)$ onto $S O(3)$. Moreover, $\Lambda^{-1}\left(\left\{1_{4}\right\}\right)=\left\{-1_{2}, 1_{2}\right\}$. 


\section{Finite Dimensional Representations of $S U(2)$}

In this section we review some well-known results. The $S U(2)$ is the group of all unitary matrices in $\mathbb{C}^{2 \times 2}$ with determinant 1 , and the group multiplication is just the ordinary matrix multiplication. Every $B \in S U(2)$ can be written as

$$
B=\left(\begin{array}{cc}
a & -\bar{b} \\
b & \bar{a}
\end{array}\right)
$$

for some $a, b \in \mathbb{C}$ with $|a|^{2}+|b|^{2}=1$. A complete system of irreducible strongly continuous unitary representations of $S U(2)$ is given by $D^{(j)}: S U(2) \rightarrow L\left(\mathcal{P}_{j}\right)$,

$$
\left(D^{(j)}(B) f\right)(z, w):=f\left(B^{-1}(z, w)\right) \quad\left(j \in \mathbb{N}_{0} / 2\right),
$$

where $\mathcal{P}_{j}$ is the set of all homogeneous polynomial of degree $2 j$ in two variables, i.e.

$$
\mathcal{P}_{j}:=\left\{(z, w) \mapsto \sum_{k=0}^{2 j} c_{k} z^{k} w^{2 j-k}: c_{0}, c_{1}, \ldots, c_{2 j} \in \mathbb{C}\right\}
$$

and $B(z, w):=\left(B_{11} z+B_{12} w, B_{21} z+B_{22} w\right)$, see, e.g., [[Fol95] Sec. 5.4]. We note that $D^{(j)}$ is not only a representation of $S U(2)$ but also a representation of $G L(2, \mathbb{C})$, i.e. the set of all invertible matrices in $\mathbb{C}^{2 \times 2}$.

Let $D$ be a strongly continuous unitary representation of $S U(2)$ on a finite dimensional vector space $V$. Define the self-adjoint matrices $L_{1}, L_{2}, L_{3}$ by

$$
D\left(\exp \left(i \alpha \sigma_{a} / 2\right)\right)=\exp \left(i \alpha L_{a}\right)
$$

The existence and uniqueness of these matrices is guaranteed by Stone's Theorem (see also Lemma 11.6.

C.1 Lemma. We have the commutator relations

$$
\left[L_{a}, L_{b}\right]=i \sum_{c=1}^{3} \varepsilon_{a b c} L_{c}
$$

Proof. Since $D$ is a direct sum of $D^{(j)} \mathrm{s}$, it suffices to consider the representation (C.1) which is differentiable. Thus

$$
L_{a}=-\left.i \partial_{\alpha} \exp \left(i \alpha L_{a}\right)\right|_{\alpha=0}=d D^{(j)}(1) \circ \sigma_{a} / 2,
$$

hence

$$
\left[L_{a}, L_{b}\right]=d D^{(j)}(1) \circ\left[\sigma_{a}, \sigma_{b}\right] / 4=d D^{(j)}(1) \circ i \sum_{c=1}^{3} \varepsilon_{a b c} \sigma_{c} / 2=i \sum_{c=1}^{3} \varepsilon_{a b c} L_{c} .
$$


The diagonalization of $L_{3}$ then leads to the following well-known Theorem (see, e.g., Mes61] or almost any book on quantum mechanics)

C.2 Theorem. Let $D$ be an irreducible strongly continuous unitary representation of $S U(2)$ on a finite dimensional vector space $V$. Then there exists a $j \in \mathbb{N}_{0} / 2$ such that $\operatorname{dim} V=2 j+1$ and there are vectors $|j,-j\rangle,|j,-j+1\rangle, \ldots,|j, j\rangle$ forming an orthonormal basis of $V$ satisfying

$$
\begin{aligned}
L_{3}|j, m\rangle & =m|j, m\rangle, \\
L_{+}|j, m\rangle & =2^{-1 / 2}((j+m+1)(j-m))^{1 / 2}|j, m+1\rangle, \\
L_{-}|j, m\rangle & =2^{-1 / 2}((j+m)(j-m+1))^{1 / 2}|j, m-1\rangle,
\end{aligned}
$$

for $m \in[j]:=\{-j,-j+1, \ldots, j\}$, where

$$
L_{ \pm}:=\frac{L_{1} \pm i L_{2}}{\sqrt{2}}
$$

In this case we denote the representation $D$ as $D^{(j)}$.

C.3 Lemma. Considering $D^{(j)}$ we have

$$
\mathbf{L}^{2}:=\sum_{k=1}^{3} L_{k}^{2}=j(j+1) I .
$$

In particular, if $D$ is a unitary representation of $S U(2)$ on a finite dimensional vector space $V$ then $\mathbf{L}^{2}$ commutes with $D$, and $D^{(j)}$ occurs $\nu$ times in the decomposition of $D$ if and only if $j(j+1)$ is an eigenvalue of $\mathbf{L}^{2}$ with multiplicity $(2 j+1) \nu$.

Proof. Using

$$
L_{1}=\frac{1}{\sqrt{2}}\left(L_{+}+L_{-}\right) \quad L_{2}=-i \frac{1}{\sqrt{2}}\left(L_{+}-L_{-}\right)
$$

and the commutations relation $\left[L_{+}, L_{-}\right]=L_{3}$ we find

$$
\mathbf{L}^{2}=L_{3}^{2}+L_{+} L_{-}+L_{-} L_{+}=L_{3}^{2}+L_{3}+2 L_{-} L_{+} .
$$

Then by Theorem C.2 it is easy to see that $\mathbf{L}^{2}|j, m\rangle=j(j+1)|j, m\rangle$. 


\section{Tensor Products of $S U(2)$ Representations}

Let $D:=D^{\left(j_{1}\right)} \otimes D^{\left(j_{2}\right)}$. The generators for $D$ are then

$$
L_{3}=L_{3}^{(1)} \otimes 1+1 \otimes L_{3}^{(2)}, \quad L_{ \pm}=L_{ \pm}^{(1)} \otimes 1+1 \otimes L_{ \pm}^{(2)},
$$

where $L_{n}^{(k)}$ are the generators for the representations $D^{\left(j_{k}\right)}$.

If $\psi^{(1)}$ is a vector of weight ${ }^{6} m_{1}$ with respect to $L_{3}^{(1)}$ and if $\psi^{(2)}$ is a vector of weight $m_{2}$ with respect to $L_{3}^{(2)}$, then $\psi^{(1)} \otimes \psi^{(2)}$ is a vector of weight $m_{1}+m_{2}$ with respect to $L_{3}$, thus we see that in the tensor representation the weights add.

The representation $D$ is (for $\min \left(j_{1}, j_{2}\right)>0$ ) reducible. By the general theory of representations of compact groups, $D$ is a direct sum of irreducible representations. Because $\left\langle j, m\left|D^{(j)}\left(e^{i \phi \sigma_{3} / 2}\right)\right| j, m^{\prime}\right\rangle=\delta_{m m^{\prime}} e^{i \phi m}$ we have

$$
D\left(e^{i \phi \sigma_{3} / 2}\right)=\operatorname{diag}\left(\alpha^{j_{1}}, \alpha^{j_{1}-1}, \ldots, \alpha^{-j_{1}}\right) \otimes \operatorname{diag}\left(\alpha^{j_{2}}, \alpha^{j_{2}-1}, \ldots, \alpha^{-j_{2}}\right), \quad \alpha:=e^{i \phi} .
$$

By looking at the highest exponent we see that $D^{\left(j_{1}+j_{2}\right)}$ must be a part of this direct sum. The second highest exponent is $j_{1}+j_{2}-1$ and it occurs two times, but one of them is already accounted for by $D^{\left(j_{1}+j_{2}\right)}$. Hence $D^{\left(j_{1}+j_{2}-1\right)}$ is a part of the direct sum. If we continue in this manner we find

$$
D^{\left(j_{1}\right)} \otimes D^{\left(j_{2}\right)} \cong \oplus_{k=\left|j_{1}-j_{2}\right|}^{j_{1}+j_{2}} D^{(k)}
$$

We now decompose $D^{(1 / 2)} \otimes D^{(J-1 / 2)}$ into $D^{(J-1)} \oplus D^{(J)}$, where $J \geq 1$. Since the weights add, a vector $|k, r\rangle$ in the $D^{(J-1)} \oplus D^{(J)}$ representation corresponds to

$$
|k, r\rangle^{\prime}=\sum_{s=-1 / 2}^{1 / 2} C_{s, r}^{(1 / 2, J-1 / 2, k)}|1 / 2, s\rangle \otimes|J-1 / 2, r-s\rangle \quad \text { for } k \in\{J-1, J\} .
$$

Usually, we omit the prime on $|k, r\rangle^{\prime}$, although $|k, r\rangle$ does not really live in $\mathbb{C}^{2} \otimes \mathbb{C}^{2 J}$. To be more precise one would consider the unitary map $T: \mathbb{C}^{2} \otimes \mathbb{C}^{2 J} \rightarrow \mathbb{C}^{2 J-1} \oplus \mathbb{C}^{2 J+1}$,

$$
T^{-1}|k, r\rangle:=\sum_{s=-1 / 2}^{1 / 2} C_{s, r}^{(1 / 2, J-1 / 2, k)}|1 / 2, s\rangle \otimes|J-1 / 2, r-s\rangle \quad \text { for } k \in\{J-1, J\} .
$$

To define the coefficients $C_{s, r}^{(1 / 2, J-1 / 2, k)}$ we must specify the highest weight vectors:

$$
\begin{aligned}
|J, J\rangle:= & \gamma|1 / 2,1 / 2\rangle \otimes|J-1 / 2, J-1 / 2\rangle, \\
|J-1, J-1\rangle:= & \alpha|1 / 2,1 / 2\rangle \otimes|J-1 / 2, J-3 / 2\rangle \\
& +\beta|1 / 2,-1 / 2\rangle \otimes|J-1 / 2, J-1 / 2\rangle,
\end{aligned}
$$

\footnotetext{
${ }^{6}$ Weight is just a synonym for eigenvalue.
} 
where $\alpha, \beta, \gamma \in \mathbb{C}$ will be defined by means of a phase convention (see below). Since $L_{+}|J-1, J-1\rangle=0$, we must have $\beta=-\alpha(2 J-1)^{1 / 2}$, and

$$
\langle J-1, J-1 \mid J-1, J-1\rangle=1
$$

implies that $|\alpha|^{2}+|\beta|^{2}=1$, i.e. $|\alpha|=(2 J)^{-1 / 2}$. Here we will use the convention [[Mes61] Eq. (XIII.109)]

$$
\langle 1 / 2,1 / 2| \otimes\langle J-1 / 2, m|| k, k\rangle \geq 0
$$

which leads to

$$
\alpha=(2 J)^{-1 / 2}, \quad \beta=-\left(\frac{2 J-1}{2 J}\right)^{1 / 2}, \quad \gamma=1 .
$$

In this convention the Wigner $3 j$ symbol satisfies

$$
C_{s, r}^{(1 / 2, J-1 / 2, k)}=(-1)^{1-J+r} \sqrt{2 k+1}\left(\begin{array}{ccc}
1 / 2 & J-1 / 2 & k \\
s & r-s & -r
\end{array}\right) .
$$

D.1 Lemma. We have

$$
C_{s, r}^{(1 / 2, J-1 / 2, J)}=\left(\frac{J+2 s r}{2 J}\right)^{1 / 2}
$$

Proof by induction on $r$. Let us abbreviate $C_{s, r}:=C_{s, r}^{(1 / 2, J-1 / 2, J)}$. Because the highest weight vector in $D^{(J)}$ is given by

$$
|J, J\rangle=|1 / 2,1 / 2\rangle \otimes|J-1 / 2, J-1 / 2\rangle,
$$

we must have $C_{1 / 2, J}=1$ and $C_{-1 / 2, J}=0$. Thus the claim is true for $r=J$. Now suppose the formula holds for some $r \leq J$. Then

$|J, r\rangle=C_{1 / 2, r}|1 / 2,1 / 2\rangle \otimes|J-1 / 2, r-1 / 2\rangle+C_{-1 / 2, r}|1 / 2,-1 / 2\rangle \otimes|J-1 / 2, r+1 / 2\rangle$. Applying $L_{-}=L_{-}^{(1 / 2)} \otimes 1+1 \otimes L_{-}^{(J-1 / 2)}$ on both sides of this equation we obtain

$$
\begin{aligned}
\left.\frac{1}{\sqrt{2}}((J) r)(J-r+1)\right)^{1 / 2}|J, r-1\rangle \\
=C_{1 / 2, r} \frac{1}{\sqrt{2}}|1 / 2,-1 / 2\rangle \otimes|J-1 / 2, r-1 / 2\rangle \\
\quad+C_{1 / 2, r} \frac{1}{\sqrt{2}}((J+r-1)(J-r+1))^{1 / 2}|1 / 2,1 / 2\rangle \otimes|J-1 / 2, r-3 / 2\rangle \\
\quad+C_{-1 / 2, r} \frac{1}{\sqrt{2}}((J+r)(J-r))^{1 / 2}|1 / 2,-1 / 2\rangle \otimes|J-1 / 2, r-1 / 2\rangle .
\end{aligned}
$$

Since

$$
\begin{aligned}
C_{-1 / 2, r-1} & =\frac{C_{1 / 2, r}+C_{-1 / 2, r}((J+r)(J-r))^{1 / 2}}{((J+r)(J-r+1))^{1 / 2}} \\
C_{1 / 2, r-1} & =\frac{C_{1 / 2, r}((J+r-1)(J-r+1))^{1 / 2}}{((J+r)(J-r+1))^{1 / 2}}
\end{aligned}
$$

the formula also holds for $C_{s, r-1}$ and the proof is complete. 
D.2 Lemma. We have

$$
C_{s, r}^{(1 / 2, J-1 / 2, J-1)}=(-1)^{s-1 / 2}\left(\frac{J-2 s r}{2 J}\right)^{1 / 2}
$$

Proof by induction on $r$. Let us abbreviate $E_{s, r}:=C_{s, r}^{(1 / 2, J-1 / 2, J-1)}$. The highest weight vector in $D^{(J-1)}$ is given by

$|J-1, J-1\rangle=\alpha|1 / 2,1 / 2\rangle \otimes|J-1 / 2, J-3 / 2\rangle+\beta|1 / 2,-1 / 2\rangle \otimes|J-1 / 2, J-1 / 2\rangle$.

Since $E_{1 / 2, J-1}=\alpha$ and $E_{-1 / 2, J-1}=\beta$ the claim is true for $r=J-1$. Now suppose the formula holds for some $r \leq J-1$. Then

$$
\begin{aligned}
|J-1, r\rangle= & E_{1 / 2, r}|1 / 2,1 / 2\rangle \otimes|J-1 / 2, r-1 / 2\rangle \\
& +E_{-1 / 2, r}|1 / 2,-1 / 2\rangle \otimes|J-1 / 2, r+1 / 2\rangle .
\end{aligned}
$$

Applying $L_{-}=L_{-}^{(1 / 2)} \otimes 1+1 \otimes L_{-}^{(J-1 / 2)}$ on both sides of the equation gives

$$
\begin{aligned}
\frac{1}{\sqrt{2}}(( & J-1+r)(J-r))^{1 / 2}|J-1, r-1\rangle \\
= & E_{1 / 2, r} \frac{1}{\sqrt{2}}|1 / 2,-1 / 2\rangle \otimes|J-1 / 2, r-1 / 2\rangle \\
& +E_{1 / 2, r} \frac{1}{\sqrt{2}}((J+r-1)(J-r+1))^{1 / 2}|1 / 2,1 / 2\rangle \otimes|J-1 / 2, r-3 / 2\rangle \\
& +E_{-1 / 2, r} \frac{1}{\sqrt{2}}((J+r)(J-r))^{1 / 2}|1 / 2,-1 / 2\rangle \otimes|J-1 / 2, r-1 / 2\rangle .
\end{aligned}
$$

Because

$$
\begin{aligned}
E_{-1 / 2, r-1} & =\frac{E_{1 / 2, r}+E_{-1 / 2, r}((J+r)(J-r))^{1 / 2}}{((J-1+r)(J-r))^{1 / 2}} \\
E_{1 / 2, r-1} & =\frac{E_{1 / 2, r}((J+r-1)(J-r+1))^{1 / 2}}{((J-1+r)(J-r))^{1 / 2}}
\end{aligned}
$$

the formula also holds for $E_{s, r-1}$ and the proof is complete. 


\section{E The Wigner $3 j$ Symbols}

We already encountered some Wigner $3 j$ symbols in Appendix D, where we decomposed $D^{(1 / 2)} \otimes D^{(J-1 / 2)}$. Considering the general case $D^{\left(j_{1}\right)} \otimes D^{\left(j_{2}\right)}$ one has the decomposition

$$
|J, M\rangle=\sum_{m_{1}, m_{2}}\left\langle j_{1} j_{2} m_{1} m_{2} \mid J M\right\rangle\left|j_{1}, m_{1}\right\rangle \otimes\left|j_{2}, m_{2}\right\rangle,
$$

where $J \in\left\{\left|j_{1}-j_{2}\right|, \ldots, j_{1}+j_{2}\right\}$ and $\left\langle j_{1} j_{2} m_{1} m_{2} \mid J M\right\rangle$ are the Clebsch-Gordon coefficients for which one usually stipulates the phase convention [[Mes61] Eq. (XIII.109)]

$$
\left\langle j_{1} j_{2} j_{1} m_{2} \mid J J\right\rangle \geq 0
$$

in addition to the $L_{ \pm}$relations of Theorem $\bar{C} .2$ for $\left|j_{1}, m_{1}\right\rangle,\left|j_{2}, m_{2}\right\rangle$ and $|J, M\rangle$. Since the weights add, we must have $\left\langle j_{1} j_{2} m_{1} m_{2} \mid J M\right\rangle=0$ if $m_{1}+m_{2} \neq M$.

Let us summarize the most important properties of the Wigner $3 j$ symbols [Wei13], [[Mes61] (C.12) and fol.]. The Wigner $3 j$ symbols are defined as

$$
\left(\begin{array}{ccc}
j_{1} & j_{2} & J \\
m_{1} & m_{2} & -M
\end{array}\right):=\frac{(-1)^{j_{1}-j_{2}+M}}{\sqrt{2 J+1}}\left\langle j_{1} j_{2} m_{1} m_{2} \mid J M\right\rangle
$$

(a) They are all real.

(b) Selection rules: If one or more of the following conditions are not satisfied then the symbol vanishes:

$$
\begin{gathered}
m_{1} \in\left[j_{1}\right], \quad m_{2} \in\left[j_{2}\right], \quad M \in[J], \\
m_{1}+m_{2}=M, \\
\left|j_{1}-j_{2}\right| \leq J \leq j_{1}+j_{2},
\end{gathered}
$$

where $[j]:=\{-j,-j+1, \ldots,+j\}$.

(c) Symmetries:

$$
(-1)^{j_{1}+j_{2}+j_{3}}\left(\begin{array}{ccc}
j_{1} & j_{2} & j_{3} \\
m_{1} & m_{2} & m_{3}
\end{array}\right)=\left(\begin{array}{ccc}
j_{\pi(1)} & j_{\pi(2)} & j_{\pi(3)} \\
m_{\pi(1)} & m_{\pi(2)} & m_{\pi(3)}
\end{array}\right)=\left(\begin{array}{ccc}
j_{1} & j_{2} & j_{3} \\
-m_{1} & -m_{2} & -m_{3}
\end{array}\right),
$$

whenever $\pi$ is an odd permutation. In particular, the symbol is invariant under an even permutation of its columns.

(d) Orthogonality relations: We have

$$
\sum_{m_{1}, m_{2}}\left(\begin{array}{ccc}
j_{1} & j_{2} & j_{3} \\
m_{1} & m_{2} & m_{3}
\end{array}\right)\left(\begin{array}{ccc}
j_{1} & j_{2} & j_{3}^{\prime} \\
m_{1} & m_{2} & m_{3}^{\prime}
\end{array}\right)=\frac{1}{2 j_{3}+1} \delta_{j_{3} j_{3}^{\prime}} \delta_{m_{3} m_{3}^{\prime}}
$$

for $m_{3} \in\left\{-j_{3}, \ldots, j_{3}\right\}$ and $\left|j_{1}-j_{2}\right| \leq j_{3} \leq j_{1}+j_{2}$. Moreover,

$$
\sum_{j_{3}, m_{3}}\left(2 j_{3}+1\right)\left(\begin{array}{ccc}
j_{1} & j_{2} & j_{3} \\
m_{1} & m_{2} & m_{3}
\end{array}\right)\left(\begin{array}{ccc}
j_{1} & j_{2} & j_{3} \\
m_{1}^{\prime} & m_{2}^{\prime} & m_{3}
\end{array}\right)=\delta_{m_{1} m_{1}^{\prime}} \delta_{m_{2} m_{2}^{\prime}}
$$

for $m_{1} \in\left\{-j_{1}, \ldots, j_{1}\right\}$ and $m_{2} \in\left\{-j_{2}, \ldots, j_{2}\right\}$. 
E.1. Some special values. For $s \in[j]$ we have

$$
\begin{aligned}
\left(\begin{array}{ccc}
j & j & 0 \\
-s & s & 0
\end{array}\right) & =\frac{(-1)^{j+s}}{\sqrt{2 j+1}} \\
\left(\begin{array}{ccc}
j & j & 1 \\
-s & s & 0
\end{array}\right) & =-(-1)^{j+s} \frac{2 s}{\sqrt{(2 j+2)(2 j+1) 2 j}} \quad \text { for } j \geq 1 / 2 \\
\left(\begin{array}{ccc}
j & j+1 & 1 \\
-s & s & 0
\end{array}\right) & =-(-1)^{j+s} \sqrt{2}\left(\frac{(j+1+s)(j+1-s)}{(2 j+3)(2 j+2)(2 j+1)}\right)^{1 / 2}
\end{aligned}
$$

see $[[$ Mes61] (C.27)].

E.2. Recursion relations for the Wigner $3 j$ symbol. We have [SG75]

$$
\begin{aligned}
j_{3} A\left(j_{3}+1\right)\left(\begin{array}{ccc}
j_{1} & j_{2} & j_{3}+1 \\
m_{1} & m_{2} & m_{3}
\end{array}\right)= & B\left(j_{3}\right)\left(\begin{array}{ccc}
j_{1} & j_{2} & j_{3} \\
m_{1} & m_{2} & m_{3}
\end{array}\right) \\
& -\left(j_{3}+1\right) A\left(j_{3}\right)\left(\begin{array}{ccc}
j_{1} & j_{2} & j_{3}-1 \\
m_{1} & m_{2} & m_{3}
\end{array}\right),
\end{aligned}
$$

where

$$
\begin{aligned}
& A\left(j_{3}\right):=\left(j_{3}^{2}-\left(j_{2}-j_{1}\right)^{2}\right)^{1 / 2}\left(\left(j_{1}+j_{2}+1\right)^{2}-j_{3}^{2}\right)^{1 / 2}\left(j_{3}^{2}-m_{3}^{2}\right)^{1 / 2}, \\
& B\left(j_{3}\right):=-\left(2 j_{3}+1\right)\left(\left(j_{2}\left(j_{2}+1\right)-j_{1}\left(j_{1}+1\right)\right) m_{3}-j_{3}\left(j_{3}+1\right)\left(m_{1}-m_{2}\right)\right) .
\end{aligned}
$$

E.3 Lemma. Let $j \in \mathbb{N}_{0} / 2$, let $n \in\{0,1, \ldots, 2 j\}$ and let $s \in[j]$, then

$$
(-1)^{j-s} s^{n}=\sum_{k=0}^{n} \alpha_{n, k, j}\left(\begin{array}{ccc}
j & j & k \\
-s & s & 0
\end{array}\right)
$$

for some $\alpha_{n, k, j} \in \mathbb{R}$.

Proof. We have

$$
\begin{aligned}
& \left(\begin{array}{ccc}
j & j & 0 \\
-s & s & 0
\end{array}\right)=(-1)^{j+s} \frac{1}{\sqrt{2 j+1}} \\
& \left(\begin{array}{ccc}
j & j & 1 \\
-s & s & 0
\end{array}\right)=-(-1)^{j+s} \frac{s}{\sqrt{j(j+1)(2 j+1)}},
\end{aligned}
$$

and the recursion relation (E.1) gives

$$
\begin{aligned}
\left(\begin{array}{ccc}
j & j & k+1 \\
-s & s & 0
\end{array}\right)= & -\frac{2(2 k+1) s}{(k+1) \sqrt{(2 j+1)^{2}-(k+1)^{2}}}\left(\begin{array}{ccc}
j & j & k \\
-s & s & 0
\end{array}\right) \\
& -\frac{k}{k+1}\left(\frac{(2 j+1)^{2}-k^{2}}{(2 j+1)^{2}-(k+1)^{2}}\right)^{1 / 2}\left(\begin{array}{ccc}
j & j & k-1 \\
-s & s & 0
\end{array}\right) .
\end{aligned}
$$

This shows that $(-1)^{j+s}\left(\begin{array}{ccc}j & j & k \\ -s & s & 0\end{array}\right)$ is a polynomial in $s$ of degree $k$ where the coefficients depend on $k$ and $j$. 
E.4 Lemma. For $l>n \in \mathbb{N}_{0}$ we have

$$
\sum_{s \in[j]} s^{n}(-1)^{j-s}\left(\begin{array}{ccc}
j & j & l \\
-s & s & 0
\end{array}\right)=0
$$

Proof. For $l>2 j$ the Lemma is trivial, since the selection rules implies that the symbols are zero. Let $0 \leq l \leq 2 j$. Using Lemma E.3 and the orthogonality relation (and $l>n$ ) we obtain

$$
\begin{aligned}
\sum_{s \in[j]} s^{n}(-1)^{j-s}\left(\begin{array}{ccc}
j & j & l \\
-s & s & 0
\end{array}\right) & =\sum_{k=0}^{n} \alpha_{n, k, j} \sum_{s \in[j]}\left(\begin{array}{ccc}
j & j & k \\
-s & s & 0
\end{array}\right)\left(\begin{array}{ccc}
j & j & l \\
-s & s & 0
\end{array}\right) \\
& =\sum_{k=0}^{n} \alpha_{n, k, j} \frac{\delta_{k l}}{2 l+1}=0 .
\end{aligned}
$$

E.5 Lemma. Let $m \in\left[\frac{1}{2}\right]$. Then

$$
\left(\begin{array}{ccc}
1 / 2 & J-1 / 2 & J-1 \\
m & s-m & -s
\end{array}\right)=2 m(-1)^{J-s+1}\left(\frac{J-2 m s}{2 J(2 J-1)}\right)^{1 / 2}, \quad s \in[J-1], J \geq 1,
$$

note that $m \in\left[\frac{1}{2}\right]$ and $s \in[J-1]$ implies $s-m \in\left[J-\frac{1}{2}\right]$. And

$$
\left(\begin{array}{ccc}
1 / 2 & J-1 / 2 & J \\
m & s-m & -s
\end{array}\right)=(-1)^{J-s+1}\left(\frac{J+2 m s}{2 J(2 J+1)}\right)^{1 / 2}, \quad s \in[J], J \geq 1 / 2,
$$

note that if $m \in\left[\frac{1}{2}\right]$ and $s \in[J]$, then $s-m \notin\left[J-\frac{1}{2}\right]$ implies $J+2 m s=0$, so there is no need to restrict $s-m$.

Proof. See Appendix D or use [[Mes61] (C.27)]. 


\section{F Causal Transformations}

Causal transformations have been introduced by Castrigiano [Cas84, they are more general than causal localizations, which are the main subject of this thesis, so we give a brief overview of the main results.

F.1 Definition. Let $(U, E)$ be a localization on a complex separable Hilbert space $\mathscr{H}$. A bounded operator $T$ commuting with $U$ is called a causal transformation if there is a compact ball $K$ of positive radius such that for each state $\psi \in \mathscr{H}$ localized within $K$, i.e. $E(K) \psi=\psi$, there is a compact region $\Delta$ such that the transformed state $T \psi$ is localized within $\Delta$, i.e. $E(\Delta) T \psi=T \psi$.

The following Lemma shows that the $\Delta$ s can be found in a uniform manner, i.e. they only depend on $K$ and do not depend on the specific state.

F.2 Lemma (Cas84 Lemma 1). Let $T$ be a causal transformation. Then there exists a compact ball $K^{\prime} \supset K$ concentric with $K$ such that for all states $\psi$ localized within $K$ we have that $T \psi$ is localized within $K^{\prime}$. In other words: $\left(I-E\left(K^{\prime}\right)\right) T E(K)=0$.

Proof. (Adapted from [Cas84]). Assume the contrary. Choose $f_{1} \in \mathscr{H}$ such that $\psi_{1}:=E(K) f_{1} \neq 0$. Then $E(K) \psi_{1}=\psi_{1}$ and since $T$ is a causal transformation, there exists a compact ball $\Delta_{1}$ of radius $\geq 1$ such that $E\left(\Delta_{1}\right) T \psi_{1}=T \psi_{1}$. Then, since we assume the claim to be false, there exists an $f_{2} \in \mathscr{H}$ such that

$$
E\left(\Delta_{1}\right) T E(K) f_{2} \neq T E(K) f_{2} .
$$

This implies that $\psi_{2}:=E(K) f_{2} \neq 0$.

Suppose $\psi_{1}, \ldots, \psi_{n}$ and $\Delta_{1}, \ldots, \Delta_{n-1}$ have been chosen. Then there exists a compact ball $\Delta_{n}$ of radius $\geq n$ such that $E\left(\Delta_{n}\right) T \psi_{n}=T \psi_{n}$. Choose $f_{n+1} \in \mathscr{H}$ such that

$$
E\left(\Delta_{n}\right) T E(K) f_{n+1} \neq T E(K) f_{n+1}
$$

and put $\psi_{n+1}:=E(K) f_{n+1} \neq 0$.

Since $\psi_{n} \neq 0$ for all $n \in \mathbb{N}$, we may assume that $\left\|\psi_{n}\right\|=1$ for all $n \in \mathbb{N}$. Also without loss of generality assume that $\|T\|=1$. For $n \in \mathbb{N}$ put

$$
\beta_{1}:=1, \quad \beta_{n+1}:=\left\|\left(I-E\left(\Delta_{n}\right)\right) T \psi_{n+1}\right\| \in(0,1], \quad \alpha_{n}:=3^{-n} \beta_{n}^{-1} \prod_{i=1}^{n} \beta_{i} .
$$

Then $\psi:=\sum_{n=1}^{\infty} \alpha_{n} \psi_{n} \in E(K) \mathscr{H}$. Since $T$ is a causal transformation, there exists a compact ball $\Delta$ of positive radius such that $E(\Delta) T \psi=T \psi$. Let $k \in \mathbb{N}$ such that 
$\Delta \subset \Delta_{k}$. Then

$$
\begin{aligned}
0 & =\left\|\left(I-E\left(\Delta_{k}\right)\right) T \psi\right\|=\left\|\sum_{n \geq k+1} \alpha_{n}\left(I-E\left(\Delta_{k}\right)\right) T \psi_{n}\right\| \\
& \geq\left\|\alpha_{k+1}\left(I-E\left(\Delta_{k}\right)\right) T \psi_{k+1}\right\|-\left\|\sum_{n \geq k+2} \alpha_{n}\left(I-E\left(\Delta_{k}\right)\right) T \psi_{n}\right\| \\
& \geq \alpha_{k+1} \beta_{k+1}-\sum_{n \geq k+2} \alpha_{n} \geq \alpha_{k+1} \beta_{k+1}-\frac{1}{2} \alpha_{k+1} \beta_{k+1}>0
\end{aligned}
$$

which is impossible. Note that $\alpha_{n}=3^{-1} \beta_{n-1} \alpha_{n-1}$ for all $n \geq 2$ and $\alpha_{j+n} \leq 3^{-n} \alpha_{j}$ for all $j, n \in \mathbb{N}$. Thus for all $j \geq 2$,

$$
\sum_{n=j}^{\infty} \alpha_{n}=\sum_{n=0}^{\infty} \alpha_{j+n} \leq \alpha_{j} \sum_{n=0}^{\infty} 3^{-n}=\frac{1}{2} \alpha_{j-1} \beta_{j-1}
$$

F.3 Lemma. Let $(V, U, E)$ be a causal localization. Then for every $t \in \mathbb{R}, V(t)$ is a causal transformation.

Proof. See 2.3 .

F.4 Lemma (cf. Cas84 Lemma 2). Let $(U, E)$ be the coordinate space representation of a finite localization and let $T$ be a causal transformation commuting with $U$. Then there exists an entire matrix-valued function $F: \mathbb{C} \rightarrow L\left(\oplus_{j}^{\prime} \nu_{j} \mathbb{C}^{2 j+1}\right)$ such that in the helicity representation

$$
T^{h}[f]=[F(|\cdot|) f]
$$

and

$$
\|F(z)\| \leq C e^{\delta|\operatorname{Im} z|} \quad \forall z \in \mathbb{C},
$$

for some constant $C>0$, where $\delta$ is the difference between the radii of $K^{\prime}$ and $K$ (cf. Lemma F.2). Moreover, $\langle k, \kappa, r|F| j, \iota, s\rangle=0$ for $r \neq s$.

Proof. (Adapted from Cas84). Since $T^{h}$ commutes with $U^{h}$, we have that $T^{h}$ commutes with $\hat{L}_{b}, \hat{L}_{b}[f]:=\left[e^{-i\langle b,\rangle} f\right]$ for all $b \in \mathbb{R}^{3}$. By Theorem G.3 there exists a measurable bounded matrix-valued function $M: \mathbb{R}^{3} \rightarrow \mathbb{C}^{m \times m}, m:=\operatorname{dim} \oplus_{j}^{\prime} \nu_{j} \mathbb{C}^{2 j+1}$ such that $T^{h}[f]=[M f]$. By Lemma F.2 we can apply Theorem 10.15. Thus there exists an entire function $\Phi: \mathbb{C}^{3} \rightarrow \mathbb{C}^{m \times m}$ such that $\left.\Phi\right|_{\mathbb{R}^{3}}=X^{-1} M X$ almost everywhere and

$$
\|\Phi(z)\| \leq C e^{\delta|\operatorname{Im} z|} \quad \forall z \in \mathbb{C}^{3},
$$

for some constant $C>0$. Let $D(B):=\oplus_{j}^{\prime} \nu_{j} D^{(j)}(B)$, then we have

$$
\Phi(\mathbf{p})=D(B(\mathbf{p})) M(\mathbf{p}) D\left(B(\mathbf{p})^{-1}\right) \quad \lambda \text {-a.e. }
$$

Since $T$ commutes with $U(0, B)$, there exists for every $B \in S U(2)$ a nullset $N_{B} \subset \mathbb{R}^{3}$ such that

$$
D(B) \Phi\left(B^{-1} \cdot \mathbf{p}\right)=\Phi(\mathbf{p}) D(B) \quad \forall \mathbf{p} \in N_{B}^{c} .
$$


By continuity this equation must hold everywhere. Then

$$
M(\mathbf{p}) \stackrel{\text { a.e. }}{=} D\left(B(\mathbf{p})^{-1}\right) \Phi(\mathbf{p}) D(B(\mathbf{p}))=\Phi\left(B(\mathbf{p})^{-1} \mathbf{p}\right)=\Phi\left(|\mathbf{p}| e_{3}\right) .
$$

Hence $F: \mathbb{C} \rightarrow \mathbb{C}^{m \times m}, F(z):=\Phi\left(z e_{3}\right)$ satisfies the first part of the Lemma. Lemma 1.13 completes the proof.

Further analysis of these entire functions reveal the main Theorem of [Cas84]:

F.5 Theorem (Castrigiano). Let $(U, E)$ be the coordinate representation of a localization. A bounded operator $T$ commuting with $U$ is a causal transformation if and only if there exists an entire matrix-valued function $F: \mathbb{C} \rightarrow \underset{j \in \Omega}{\oplus} \nu_{j} L\left(\mathbb{C}^{2 j+1}\right)$ such that in the helicity representation $T^{h}[g]=[F(|\cdot|) g]$ for all $[g] \in L^{2}\left(\mathbb{R}^{3}, \mathbb{C}^{m}\right)$ and

$$
\langle k, \kappa, r|F(\rho)| j, \iota, s\rangle=\delta_{r s} \sum_{l}(-1)^{j-s} \sqrt{2 l+1}\left(\begin{array}{rrr}
j & k & l \\
-s & s & 0
\end{array}\right) \rho^{l}\left(f_{j k l}\left(\rho^{2}\right)\right)_{\kappa \iota} \quad \forall \rho \geq 0,
$$

where $f_{j k l}$ are entire matrix-valued functions of one complex variable such that $z \mapsto$ $f_{j k l}\left(z^{2}\right)$ are uniformly exponentially bounded and $\left(\begin{array}{rrr}j & k & l \\ -s & s & 0\end{array}\right)$ is the Wigner $3 j$ symbol. 


\section{G Auxiliary Lemmata}

The purpose of this section is to provide theorems and lemmata needed in the proofs of section 1. I assume that they are well-known, however for the most of them I could not find any references giving proofs.

G.1 Theorem. Let $T$ be a bounded operator on $L^{2}\left(\mathbb{R}^{d}, \mathbb{C}^{m}, \lambda\right)$, where $\lambda$ is the Lebesgue measure on $\mathbb{R}^{d}$. Then $E(\Delta) T=T E(\Delta)$ for all $\Delta \in \mathscr{B}\left(\mathbb{R}^{d}\right)$, where $E: \mathscr{B}\left(\mathbb{R}^{d}\right) \rightarrow$ $L\left(L^{2}\left(\mathbb{R}^{d}, \mathbb{C}, \lambda\right)\right)$ is the canonical projection valued measure, i.e. $E(\Delta)[f]=\left[\chi_{\Delta} f\right], \chi_{\Delta}$ being the characteristic function of $\Delta$, if and only if there exists an $A \in L^{\infty}\left(\mathbb{R}^{d}, \mathbb{C}^{m \times m}, \lambda\right)$ such that $T[f]=[A f]$.

Proof. For the "only if" part of the Theorem consider first the case for $m=1$.

Let $K_{1}, K_{2}, \ldots$ be a sequence of compact sets such that $K_{n} \subset K_{n+1}$ and $\bigcup_{n \in \mathbb{N}} K_{n}=$ $\mathbb{R}^{d}$. Let $A_{1}: \mathbb{R}^{d} \rightarrow \mathbb{C}$ be a representative of $\left[T \chi_{K_{1}}\right]$. If $A_{1}, \ldots, A_{n}$ have been chosen, let $A_{n+1}: \mathbb{R}^{d} \rightarrow \mathbb{C}$ be a representative of $\left[T \chi_{K_{n+1}}\right]$ such that

$$
A_{n+1}(p)=A_{n}(p) \quad \forall p \in K_{n},
$$

which is possible because

$$
\chi_{K_{n}} A_{n+1}=E\left(K_{n}\right) T \chi_{K_{n+1}}=T E\left(K_{n}\right) \chi_{K_{n+1}}=T \chi_{K_{n}}=A_{n}, \quad \lambda \text {-a.e. }
$$

Put $A(p):=\lim _{n \rightarrow \infty} A_{n}(p)$. Since $A$ is the pointwise limit of a sequence of measurable functions, it is measurable. We show that $A$ is essentially bounded, i.e. $\lambda\left(S_{\beta}\right)=0$ for some $\beta>0$, where $S_{\beta}:=\left\{p \in \mathbb{R}^{d}:|A(p)|>\beta\right\}$. Put $S_{\beta}^{(n)}:=\left\{p \in \mathbb{R}^{d}:\left|A_{n}(p)\right|>\beta\right\}$. Then we have

$$
\begin{aligned}
\beta^{2} \lambda\left(S_{\beta}^{(n)}\right) & \leq \int\left|A_{n}\right|^{2} \chi_{S_{\beta}^{(n)}} d \lambda=\int\left|E\left(S_{\beta}^{(n)}\right) T \chi_{K_{n}}\right|^{2} d \lambda=\left\|T \chi_{S_{\beta}^{(n)} \cap K_{n}}\right\|^{2} \\
& \leq\|T\|^{2}\left\|\chi_{S_{\beta}^{(n)} \cap K_{n}}\right\|^{2} \leq\|T\|^{2} \lambda\left(S_{\beta}^{(n)}\right) .
\end{aligned}
$$

Hence $\lambda\left(S_{\beta}^{(n)}\right)=0$ for all $n \in \mathbb{N}$ if $\beta>\|T\|$, whence $\lambda\left(S_{\beta}\right)=0$ for $\beta>\|T\|$.

Thus $T^{\prime}[f]:=[A f]$ defines a linear bounded operator on $L^{2}\left(\mathbb{R}^{d}, \mathbb{C}, \lambda\right)$. It remains to show that $T=T^{\prime}$. Let $\Delta \in \mathscr{B}\left(\mathbb{R}^{d}\right)$ be a set of finite measure. For $\varepsilon>0$ choose $n \in \mathbb{N}$ such that $\lambda\left(\Delta \cap K_{n}^{c}\right)<\varepsilon$. Put $\Delta_{n}:=\Delta \cap K_{n}$, then

$$
\begin{aligned}
\left\|T \chi_{\Delta}-T^{\prime} \chi_{\Delta}\right\| & =\left\|\left(T-T^{\prime}\right)\left(\chi_{\Delta \cap K_{n}}+\chi_{\Delta \cap K_{n}^{c}}\right)\right\| \\
& \leq\left\|\left(T-T^{\prime}\right) \chi_{\Delta_{n}}\right\|+\left\|T-T^{\prime}\right\| \varepsilon^{1 / 2},
\end{aligned}
$$

and since

$$
\begin{aligned}
\left\|\left(T-T^{\prime}\right) \chi_{\Delta_{n}}\right\| & =\left\|T \chi_{\Delta_{n}}-A_{n} \chi_{\Delta_{n}}\right\|=\left\|T E\left(\Delta_{n}\right) \chi_{K_{n}}-A_{n} \chi_{\Delta_{n}}\right\| \\
& =\left\|E\left(\Delta_{n}\right) A_{n}-A_{n} \chi_{\Delta_{n}}\right\|=0
\end{aligned}
$$


we have $T \chi_{\Delta}=T^{\prime} \chi_{\Delta}$. This shows that $T g=T^{\prime} g$ for all simple functions $g$, and since these are dense in $L^{2}\left(\mathbb{R}^{d}, \mathbb{C}, \lambda\right)$, we have $T=T^{\prime}$.

For the case $m>1$ let $\left\{b_{1}, \ldots, b_{m}\right\}$ be an orthonormal basis in $\mathbb{C}^{m}$. For $1 \leq$ $i, j \leq m$ define the operators $T_{i j}$ on $L^{2}\left(\mathbb{R}^{d}, \mathbb{C}, \lambda\right)$ by

$$
T_{i j}[f]:=\left\langle b_{i}, T\left[f b_{j}\right]\right\rangle .
$$

Then $\left[T_{i j}, E^{\prime}(\Delta)\right]=0$ for all $\Delta \in \mathscr{B}\left(\mathbb{R}^{d}\right)$, where $E^{\prime}(\Delta)[f]:=\left[\chi_{\Delta} f\right]$. By the case for $m=1$ there exist $A_{i j} \in L^{\infty}\left(\mathbb{R}^{d}, \mathbb{C}, \lambda\right)$ such that $T_{i j}[f]=\left[A_{i j} f\right]$ for all $[f] \in L^{2}\left(\mathbb{R}^{d}, \mathbb{C}, \lambda\right)$. This implies $T[f]=[A f]$ for all $[f] \in L^{2}\left(\mathbb{R}^{d}, \mathbb{C}^{m}, \lambda\right)$.

The "if" part of the Theorem is trivial.

G.2 Corollary. Let $T$ be a bounded operator on $L^{2}\left(\mathbb{R}^{d}, \mathbb{C}^{m}\right)$ and let $E: \mathscr{B}\left(\mathbb{R}^{d}\right) \rightarrow$ $L\left(L^{2}\left(\mathbb{R}^{d}, \mathbb{C}^{m}, \lambda\right)\right)$ be the canonical projection-valued measure. Then $E(S) T=T E(S)$ for all $S \in \mathscr{O}$, where $\mathscr{O}$ denotes the set of all open orthotopes in $\mathbb{R}^{d}$, if and only if there exists an $A \in L^{\infty}\left(\mathbb{R}^{d}, \mathbb{C}^{m \times m}, \lambda\right)$ such that $T[f]=[A f]$.

Proof. Clearly, $\mathscr{O} \cup\{\varnothing\}$ is stable under finitely many intersections and we have

$$
\mathscr{B}\left(\mathbb{R}^{d}\right)=\sigma(\mathscr{O})=\delta(\mathscr{O}),
$$

where $\delta(\mathscr{O})$ is the smallest Dynkin system containing $\mathscr{O}$. It remains to show that

$$
\mathscr{G}:=\left\{\Delta \in \mathscr{B}\left(\mathbb{R}^{d}\right): E(\Delta) T=T E(\Delta)\right\}
$$

is a Dynkin system, since then $\mathscr{B}\left(\mathbb{R}^{d}\right)=\delta(\mathscr{O}) \subset \mathscr{G} \subset \mathscr{B}\left(\mathbb{R}^{d}\right)$.

Obviously $\mathbb{R}^{d} \in \mathscr{G}$. If $\Delta \in \mathscr{G}$, then

$$
T E\left(\Delta^{c}\right)=T(I-E(\Delta))=(I-E(\Delta)) T=E\left(\Delta^{c}\right) T
$$

thus $\Delta^{c} \in \mathscr{G}$. If $\Delta_{1}, \Delta_{2}, \ldots$ is a sequence of mutually disjoint sets in $\mathscr{G}$, then for $f \in L^{2}\left(\mathbb{R}^{d}, \mathbb{C}^{m}, \lambda\right)$ we have

$$
T E\left(\cup_{n} \Delta_{n}\right) f=T \sum_{n} E\left(\Delta_{n}\right) f=\sum_{n} T E\left(\Delta_{n}\right) f=\sum_{n} E\left(\Delta_{n}\right) T f=E\left(\cup_{n} \Delta_{n}\right) T f,
$$

hence $\cup_{n} \Delta_{n} \in \mathscr{G}$.

G.3 Lemma. For $t \in \mathbb{R}$ and $k \in\{1,2, \ldots, d\}$ define $U_{k}(t) \in L\left(L^{2}\left(\mathbb{R}^{d}, \mathbb{C}^{m}, \lambda\right)\right)$ as

$$
U_{k}(t)[f]:=\left[e^{i t\left\langle e_{k}, \cdot\right\rangle} f\right] .
$$

Then a bounded operator $T$ commutes with all $U_{k}$ if and only if there exists an $A \in$ $L^{\infty}\left(\mathbb{R}^{d}, \mathbb{C}^{m \times m}, \lambda\right)$ such that $T[f]=[A f]$. 
Proof. The "if" part of the Theorem is trivial. The "only if" part of the Theorem: Clearly, $U_{k}$ is a continuous unitary one parameter group. The self-adjoint generator $H_{k}$ for $U_{k}$ is then given by

$$
\mathscr{D}\left(H_{k}\right)=\left\{[f] \in L^{2}:\left[\left\langle e_{k}, \cdot\right\rangle f(\cdot)\right] \in L^{2}\right\}, \quad H_{k}[f]:=\left[\left\langle e_{k}, \cdot\right\rangle f(\cdot)\right] .
$$

If $[f] \in \mathscr{D}\left(H_{k}\right)$ then the limit $t^{-1}\left(U_{k}(t)-I\right) T[f]$ for $t \rightarrow 0$ exists, since $T$ commutes with $U_{k}$. Hence, $T \mathscr{D}\left(H_{k}\right) \subset \mathscr{D}\left(H_{k}\right)$ and $\left[T, H_{k}\right][f]=0$, whence $T H_{k} \subset H_{k} T$. Let $E_{k}: \mathscr{B}(\mathbb{R}) \rightarrow L\left(L^{2}\right)$ be the projection-valued measure defined as

$$
E_{k}(M):=E\left(\left\{\left(x_{1}, \ldots, x_{d}\right) \in \mathbb{R}^{d}: x_{k} \in M\right\}\right),
$$

where $E: \mathscr{B}\left(\mathbb{R}^{d}\right) \rightarrow L\left(L^{2}\right)$ is the canonical projection-valued measure, i.e.

$$
E(\Delta)[f]:=\left[\chi_{\Delta} f\right]
$$

Since $\int u d E_{k}[f]=\left[u\left(\left\langle e_{k}, \cdot\right\rangle\right) f\right]$ for every simple function $u: \mathbb{R} \rightarrow \mathbb{C}$, we have

$$
H_{k}=\int \mathrm{id} d E_{k}
$$

By the Spectral Theorem [ $\left[\right.$ Cas11] Ch.5 (7) p. 68] we have $\left[T, E_{k}\right]=0$. Considering that

$$
E\left(\left(a_{1}, b_{1}\right) \times \ldots \times\left(a_{d}, b_{d}\right)\right)=\prod_{k=1}^{d} E_{k}\left(\left(a_{k}, b_{k}\right)\right),
$$

we have $[T, E(S)]=0$ for all open orthotopes $S$ in $\mathbb{R}^{d}$. By Corollary G.2 the proof is complete.

G.4 Lemma. Let $\lambda$ be the Lebesgue measure on $\mathbb{R}^{d}$. For $y \in \mathbb{R}^{d}$ let $L_{y}$ be the left translation operator on $L^{\infty}\left(\mathbb{R}^{d}, \mathbb{C}^{m \times m}\right)$, i.e.

$$
L_{y}[f]:=[f(\cdot+y)]
$$

Then $L_{y}[f]=[f]$ for all $y \in \mathbb{R}^{d}$, if and only if $f$ is constant almost everywhere.

Note. Let $y \in \mathbb{R}^{d}$, then $L_{y}[f]=[f]$ means, that there exists a set $N_{y}$ of measure zero such that $f(x)=f(x+y)$ for all $x \in \mathbb{R}^{d} \backslash N_{y}$.

Proof. The "if" part of the Theorem is trivial. For the "only if" part of the Theorem consider first the case for $m=1$. The case $m>1$ then follows easily, since every component of $f$ must be constant almost everywhere.

Let $f \in L^{\infty}\left(\mathbb{R}^{d}, \mathbb{C}, \lambda\right)$ such that $L_{y}[f]=[f]$ for all $y \in \mathbb{R}^{d}$. Then by means of $f=$ $u_{+}-u_{-}+i\left(v_{+}-v_{-}\right)$, where $u_{ \pm}(x)=\max \{ \pm \operatorname{Re} f(x), 0\}$ and $v_{ \pm}(x)=\max \{ \pm \operatorname{Im} f(x), 0\}$, we see that $L_{y}\left[u_{ \pm}\right]=\left[u_{ \pm}\right]$and $L_{y}\left[v_{ \pm}\right]=\left[v_{ \pm}\right]$, hence we may assume without loss of generality that $f$ is nonnegative.

Let $I:=[0,1]^{d}$. Put

$$
c:=\int_{I} f(a) d \lambda(a)
$$


Note that

$$
c=\int_{I} f(a+x) d \lambda(a) \quad \forall x \in \mathbb{R}^{d} .
$$

Let $K \subset \mathbb{R}^{d}$ be a compact set. Since

$$
\left.\int_{K} \mid f(a+x)-f(x)\right) \mid d \lambda(x)=0
$$

for every $a \in \mathbb{R}^{d}$, the Fubini-Tonelli Theorem implies

$$
\begin{aligned}
0 \leq \int_{K}|c-f(x)| d \lambda(x) & =\int_{K}\left|\int_{I}(f(a+x)-f(x)) d \lambda(a)\right| d \lambda(x) \\
& \left.\leq \int_{K} \int_{I} \mid f(a+x)-f(x)\right) \mid d \lambda(a) d \lambda(x) \\
& \left.=\int_{I} \int_{K} \mid f(a+x)-f(x)\right) \mid d \lambda(x) d \lambda(a)=0 .
\end{aligned}
$$

Hence $f=c$ almost everywhere.

G.5 Lemma. Let $\lambda$ be the Lebesgue measure on $\mathbb{R}^{d}$ and let $A: \mathbb{R}^{d} \rightarrow \mathbb{C}^{m \times m}$ be a measurable matrix-valued function. If $A[f]=[0]$ for all $f \in C_{c}^{\infty}\left(\mathbb{R}^{d}, \mathbb{C}^{m}\right)$ then $A=0$ almost everywhere.

Proof. Fix $1 \leq i, j \leq m$ and assume that $A_{i j}=0$ not almost everywhere. Then $\lambda(S)>0$ where

$$
S:=\left\{x \in \mathbb{R}^{d}:\left|A_{i j}(x)\right|>0\right\}
$$

For $n \in \mathbb{N}$ put

$$
S_{0}:=\left\{x \in \mathbb{R}^{d}:\left|A_{i j}(x)\right|>1\right\} \quad S_{n}:=\left\{x \in \mathbb{R}^{d}: \frac{1}{n+1}<\left|A_{i j}(x)\right| \leq \frac{1}{n}\right\} .
$$

Then $S_{i} \cap S_{j}=\varnothing$ for $i \neq j$ and $S=\cup_{n=0}^{\infty} S_{n}$. Since $0<\lambda(S)=\sum_{n=0}^{\infty} \lambda\left(S_{n}\right)$, there exists an $n \in \mathbb{N}_{0}$ such that $0<\lambda\left(S_{n}\right)$. Then there exists a measurable bounded set $R \subset S_{n}$ such that $0<\lambda(R)<\infty$. Let $f \in C_{c}^{\infty}\left(\mathbb{R}^{d}\right)$ such that $\left.f\right|_{R} \geq 1$. But then

$$
0=\left\|A f e_{j}\right\|^{2} \geq \int_{R}\left|A_{i j}\right|^{2} d \lambda \geq \lambda(R)(n+1)^{-2}>0
$$

is a contradiction. Hence $A_{i j}=0$ a.e., whence $A=0$ a.e.

G.6 Lemma. Let $T \in L\left(L^{2}\left(\mathbb{R}^{3}, \mathbb{C}^{m}\right)\right), T[f]:=[\Phi f]$, for some measurable and bounded matrix-valued function $\Phi: \mathbb{R}^{3} \rightarrow \mathbb{C}^{m \times m}$. Let $U: S U(2) \rightarrow L\left(L^{2}\left(\mathbb{R}^{3}, \mathbb{C}^{m}\right)\right)$,

$$
U(B)[f]:=\left[f\left(B^{-1} \cdot\right)\right] .
$$

Then $T$ commutes with $U$ if and only if there exists a measurable bounded function $\tilde{\Phi}: \mathbb{R}_{\geq 0} \rightarrow \mathbb{C}^{m \times m}$ such that

$$
\Phi(\mathbf{p})=\tilde{\Phi}(|\mathbf{p}|) \quad \text { a.e. }
$$


Proof. The "if" part of the Theorem is trivial. By considering the operators $T_{i j}$ on $L^{2}\left(\mathbb{R}^{3}, \mathbb{C}\right)$ given by

$$
T_{i j}[f]:=\left\langle b_{i},\left[\Phi f b_{j}\right]\right\rangle=\left[\Phi_{i j} f\right],
$$

where $\left\{b_{1}, \ldots, b_{m}\right\}$ is an orthonormal basis in $\mathbb{C}^{m}$ and $\Phi_{i j}:=\left\langle b_{i}, \Phi b_{j}\right\rangle$, we see that it suffices to prove the case for $m=1$.

Put $\tilde{\Phi}: \mathbb{R}_{\geq 0} \rightarrow \mathbb{C}$

$$
\tilde{\Phi}(\rho):=\int_{S U(2)} \Phi\left(B^{-1} \cdot \rho e_{3}\right) d \mu(B),
$$

where $\mu$ denotes the normalized left Haar measure of $S U(2)$. Obviously $\tilde{\Phi}$ is bounded, and since $(\rho, B) \mapsto B^{-1} \cdot \rho e_{3}$ is continuous, $(\rho, B) \mapsto \Phi\left(B^{-1} \cdot \rho e_{3}\right)$ is $\mathscr{B}\left(\mathbb{R}_{\geq 0}\right) \otimes \mathscr{B}(S U(2))$ measurable. By Fubini's Theorem $\tilde{\Phi}$ is measurable.

For each $\mathbf{p} \in \mathbb{R}^{3}$ there exists a $B^{\prime} \in S U(2)$ such that $|\mathbf{p}| e_{3}=B^{\prime} \cdot \mathbf{p}$. Then by the invariance of the Haar measure we have

$$
\tilde{\Phi}(|\mathbf{p}|)=\int_{S U(2)} \Phi\left(B^{-1} B^{\prime} \cdot \mathbf{p}\right) d \mu(B)=\int_{S U(2)} \Phi\left(B^{-1} \cdot \mathbf{p}\right) d \mu(B) \quad \forall \mathbf{p} \in \mathbb{R}^{3} .
$$

Let $f_{R}$ denote the characteristic function of the ball with radius $R>0$ centered at the origin. Then for every $B \in S U(2)$ we find

$$
\begin{aligned}
0 \leq \int\left|\Phi\left(B^{-1} \cdot\right)-\Phi\right| f_{R} d \lambda & =\left\langle\left|\Phi\left(B^{-1} \cdot\right)-\Phi\right| f_{R}, f_{R}\right\rangle \leq\left\|\left(\Phi\left(B^{-1} \cdot\right)-\Phi\right) f_{R}\right\|\left\|f_{R}\right\| \\
& =\left\|[U(B), T] f_{R}\right\|\left\|f_{R}\right\|=0 .
\end{aligned}
$$

Hence, by Fubini-Tonelli

$$
\begin{aligned}
0 \leq \int|\tilde{\Phi}(|\cdot|)-\Phi| f_{R} d \lambda & =\int\left|\int_{S U(2)}\left(\Phi\left(B^{-1} \cdot\right)-\Phi\right) d \mu(B)\right| f_{R} d \lambda \\
& \leq \iint_{S U(2)}\left|\Phi\left(B^{-1} \cdot\right)-\Phi\right| f_{R} d \mu(B) d \lambda \\
& =\int_{S U(2)} \int\left|\Phi\left(B^{-1} \cdot\right)-\Phi\right| f_{R} d \lambda d \mu(B)=0 .
\end{aligned}
$$

This implies $\tilde{\Phi}(|\cdot|)=\Phi$ a.e.

G.7 Lemma. Let $\mu$ be a $\sigma$-finite measure on $\mathscr{B}\left(\mathbb{R}^{d}\right)$. If $\mu$ is quasi-invariant under translations, i.e. $\mu(N)=0$ implies $\mu(N+a)=0$ for every $a \in \mathbb{R}^{d}$, then $\mu$ is equivalent to the Lebesgue measure $\lambda$.

Proof. (cf. [[Wig62 Appendix III.]) Let $\nu$ be another $\sigma$-finite quasi-invariant measure on $\mathscr{B}\left(\mathbb{R}^{d}\right)$ and let $N \in \mathscr{B}\left(\mathbb{R}^{d}\right)$ be a $\nu$ null set. Since

$$
0=\nu(N)=\nu(N-x)=\int \chi_{N-x}(y) d \nu(y) \quad \forall x \in \mathbb{R}^{d}
$$


where $\chi_{A}$ denotes the characteristic function of $A$, Fubini-Tonelli implies

$$
\begin{aligned}
0 & =\int\left(\int \chi_{N-x}(y) d \nu(y)\right) d \mu(x) \\
& =\iint \chi_{N}(x+y) d \nu(y) d \mu(x)=\iint \chi_{N}(x+y) d \mu(x) d \nu(y) \\
& =\iint \chi_{N-y}(x) d \mu(x) d \nu(y)=\int \mu(N-y) d \nu(y)
\end{aligned}
$$

Therefore $\mu(N-(\cdot))=0 \nu$-a.e. and there exists a $y_{0} \in \mathbb{R}^{d}$ such that $\mu\left(N-y_{0}\right)=0$. Hence $\mu(N)=\mu\left(N-y_{0}+y_{0}\right)=0$.

By interchanging $\mu$ and $\nu$, the same reasoning shows that $\mu(N)=0$ implies $\nu(N)=0$. Hence $\mu$ and $\nu$ are equivalent. Since the Lebesgue measure is $\sigma$-finite and quasi-invariant under translations, the proof is complete. 


\section{H Nevanlinna Theory and Related Results}

H.1 Lemma. We have

$$
\int_{0}^{2 \pi} \ln \sin (\theta / 2) d \theta=-2 \pi \ln 2
$$

Proof. (cf. [[Rud70 15.17 p. 299] where an equivalent formula is proved in a different manner.) It is easy to see that the integral exists, for example the right hand side of $\left|\int_{\varepsilon}^{1} \ln \sin (\theta / 2) d \theta\right| \leq-\int_{\varepsilon}^{1} \ln (\theta / 4) d \theta$ converges for $\varepsilon \rightarrow 0$. Let $I$ denote the integral, then

$$
\begin{aligned}
I & =\frac{1}{2} \int_{0}^{2 \pi} \ln \sin ^{2}(\theta / 2) d \theta=\int_{0}^{\pi} \ln \sin ^{2}(\theta) d \theta=\int_{0}^{\pi} \ln ((1-\cos \theta)(1+\cos \theta)) d \theta \\
& =\int_{0}^{\pi} \ln (1-\cos \theta) d \theta+\int_{0}^{\pi} \ln (1+\cos \theta) d \theta=2 \int_{0}^{\pi} \ln (1-\cos \theta) d \theta \\
& =2 \int_{0}^{\pi} \ln \left(2 \sin ^{2} \theta / 2\right) d \theta=2 \pi \ln 2+4 \int_{0}^{\pi} \ln \sin (\theta / 2) d \theta=2 \pi \ln 2+2 I,
\end{aligned}
$$

hence $I=-2 \pi \ln 2$.

H.2 Lemma. Let $(X, d)$ be a metric space. If $U \subset X$ is an open set containing a compact set $K$, then there exists an $\varepsilon>0$ such that

$$
\bigcup_{x \in K} B_{\varepsilon}(x) \subset U
$$

where $B_{\varepsilon}(x):=\{y \in X: d(x, y)<\varepsilon\}$.

Proof. It suffices to show that there exists an $\varepsilon>0$ such that $B_{\varepsilon}(x) \subset U$ for all $x \in K$. Suppose the contrary. Then for every $n \in \mathbb{N}$ there exists an $x_{n} \in K$ such that $B_{1 / n}\left(x_{n}\right)$ is not a subset of $U$. Since $K$ is compact, there exists a converging subsequence $\left(x_{n_{k}}\right)_{k \in \mathbb{N}}$ whose limit, denoted by $x$, is an element in $K$. Since $U$ is open, there exists a $\delta>0$ such that $B_{\delta}(x) \subset U$. Then there exists a $k \in \mathbb{N}$ such that $x_{n_{k}} \in B_{\delta / 3}(x)$ and $1 / n_{k}<\delta / 3$. But then $B_{1 / n_{k}}\left(x_{n_{k}}\right) \subset B_{\delta}(x) \subset U$.

H.3. A Brief Introduction to Nevanlinna Theory. In this number we collect some well known results on meromorphic functions in order to prove Lemma H.4. The hurried reader can skip this number, since the Lemma, although in a slightly different form, can be found in [[Lev96] Lecture 2 (13) fol.].

Poisson Formula. Let $f$ be analytic in a region $\Omega$ containing $\bar{B}_{r}(0)$, then for $z=$ $R e^{i \phi} \in B_{r}(0)$ we have

$$
\operatorname{Re} f\left(R e^{i \phi}\right)=\frac{1}{2 \pi} \int_{0}^{2 \pi} \frac{r^{2}-R^{2}}{r^{2}-2 r R \cos (\phi-\theta)+R^{2}} \operatorname{Re} f\left(r e^{i \theta}\right) d \theta .
$$


Note that

$$
\begin{aligned}
\operatorname{Re}\left(\frac{r e^{i \theta}+R e^{i \phi}}{r e^{i \theta}-R e^{i \phi}}\right) & =\operatorname{Re}\left(\frac{1+R / r e^{i(\phi-\theta)}}{1-R / r e^{i(\phi-\theta)}}\right) \\
& =\frac{1-(R / r)^{2}}{1-2 R / r \cos (\theta-\phi)+(R / r)^{2}}=\frac{r^{2}-R^{2}}{r^{2}-2 r R \cos (\phi-\theta)+R^{2}}
\end{aligned}
$$

Proof. (Adapted from [Hol73] Theorem 3.2.1, p. 42) By Lemma H.2 there exists an $r^{\prime}>r$ such that $B_{r^{\prime}}(0) \subset \Omega$. Thus $f$ has a power series representation

$$
f(z)=\sum_{n=0}^{\infty}\left(\alpha_{n}+i \beta_{n}\right) z^{n} \quad \forall z \in B_{r^{\prime}}(0),
$$

for some $\alpha_{n}, \beta_{n} \in \mathbb{R}$, cf. [[Rud70] Theorem 10.16, p. 208]. For $R<r$ and $\phi \in[0,2 \pi)$ we have

$$
f\left(R e^{i \phi}\right)=\sum_{n=0}^{\infty}\left(\alpha_{n}+i \beta_{n}\right) R^{n} e^{i n \phi} .
$$

Put $u\left(R e^{i \phi}\right):=\operatorname{Re} f\left(R e^{i \phi}\right)$, then

$$
\begin{gathered}
u\left(R e^{i \phi}\right)=\sum_{n=0}^{\infty}\left(\alpha_{n} \cos (n \phi)-\beta_{n} \sin (n \phi)\right) R^{n}, \\
\alpha_{0}=\frac{1}{2 \pi} \int_{0}^{2 \pi} u\left(r e^{i \theta}\right) d \theta
\end{gathered}
$$

and

$$
\begin{array}{rlrl}
\alpha_{n} r^{n} & =\frac{1}{\pi} \int_{0}^{2 \pi} u\left(r e^{i \theta}\right) \cos (n \theta) d \theta & \forall n \in \mathbb{N}, \\
\beta_{n} r^{n}=-\frac{1}{\pi} \int_{0}^{2 \pi} u\left(r e^{i \theta}\right) \sin (n \theta) d \theta & \forall n \in \mathbb{N} .
\end{array}
$$

Thus

$$
\begin{aligned}
u\left(R e^{i \phi}\right)= & \frac{1}{2 \pi} \int_{0}^{2 \pi} u\left(r e^{i \theta}\right) d \theta \\
& +\frac{1}{\pi} \sum_{n=1}^{\infty} \frac{R^{n}}{r^{n}} \int_{0}^{2 \pi} u\left(r e^{i \theta}\right)(\cos (n \phi) \cos (n \theta)+\sin (n \phi) \sin (n \theta)) d \theta \\
= & \frac{1}{\pi} \int_{0}^{2 \pi} u\left(r e^{i \theta}\right)\left(\frac{1}{2}+\sum_{n=1}^{\infty} \cos (n(\phi-\theta)) \frac{R^{n}}{r^{n}}\right) d \theta
\end{aligned}
$$

where we used the uniform convergence to interchange summation and integration. Now let $\gamma:=\phi-\theta$ and $x:=R / r$, then

$$
\frac{1}{2}+\sum_{n=1}^{\infty} \cos (n \gamma) x^{n}=\operatorname{Re}\left(\frac{1}{2}+\sum_{n=1}^{\infty}\left(x e^{i \gamma}\right)^{n}\right)=\frac{1}{2} \operatorname{Re}\left(\frac{1+x e^{i \gamma}}{1-x e^{i \gamma}}\right)
$$


The Poisson-Jensen Formula. Let $f$ be analytic in a region $\Omega$ which contains $\bar{B}_{r}(0)$ and let $a_{1}, \ldots, a_{n}$ be the zeros of $f$ in $B_{r}(0)$ repeated according to multiplicity. If $|z|<r$ and $f(z) \neq 0$ then

$$
\ln |f(z)|=-\sum_{k=1}^{n} \ln \left|\frac{r^{2}-\overline{a_{k}} z}{r\left(z-a_{k}\right)}\right|+\frac{1}{2 \pi} \int_{0}^{2 \pi} \operatorname{Re}\left(\frac{r e^{i \theta}+z}{r e^{i \theta}-z}\right) \ln \left|f\left(r e^{i \theta}\right)\right| d \theta .
$$

Note that if $f \neq 0$ is analytic in a bounded region $\Omega$, then $f$ has only finitely many zeros in $\Omega$, otherwise the zeros of $f$ would have a limit point $z_{0}$ and by continuity $f\left(z_{0}\right)=0$, by the Identity Theorem $f=0$.

Proof. [Adapted from [Hol73 3.4, p. 47] If $f$ has no zeros in $\bar{B}_{r}(0)$, then there exists an $r^{\prime}>r$ such that $B_{r^{\prime}}(0) \subset \Omega$ and $f(z) \neq 0$ for all $z \in B_{r^{\prime}}(0)$. Then [ [Con78] Corollary IV.6.17 p. 94] implies that there exists an analytic function $g: B_{r^{\prime}}(0) \rightarrow \mathbb{C}$ such that $f(z)=e^{g(z)}$ for all $z \in B_{r^{\prime}}(0)$. Thus $|f(z)|=e^{\operatorname{Re} g(z)}$, hence $\ln |f|$ is the real part of a function analytic in a region containing $\bar{B}_{r}(0)$. The Poisson Formula implies

$$
\ln |f(z)|=\frac{1}{2 \pi} \int_{0}^{2 \pi} \operatorname{Re}\left(\frac{r e^{i \theta}+z}{r e^{i \theta}-z}\right) \ln \left|f\left(r e^{i \theta}\right)\right| d \theta .
$$

Now let $a_{1}, \ldots, a_{n}$ be the zeros of $f$ in $B_{r}(0)$ repeated according to multiplicity. Then by [[Con78] Corollary IV.3.9 p. 79] there exists an analytic function $g: B_{r}(0) \rightarrow \mathbb{C}$ such that $g(z) \neq 0$ for all $z \in B_{r}(0)$ and

$$
f(z)=g(z) \prod_{k=1}^{n}\left(z-a_{k}\right) \quad \forall z \in B_{r}(0) .
$$

Applying the previous step to $g$, it remains to show that

$$
\ln |z-a|=-\ln \left|\frac{r^{2}-\bar{a} z}{r(z-a)}\right|+\frac{1}{2 \pi} \int_{0}^{2 \pi} \operatorname{Re}\left(\frac{r e^{i \theta}+z}{r e^{i \theta}-z}\right) \ln \left|r e^{i \theta}-a\right| d \theta,
$$

for $z, a \in B_{r}(0), z \neq a$. It suffices to prove that

$$
\ln \left|r-\frac{\bar{a} z}{r}\right|=\frac{1}{2 \pi} \int_{0}^{2 \pi} \operatorname{Re}\left(\frac{r e^{i \theta}+z}{r e^{i \theta}-z}\right) \ln \left|r e^{i \theta}-a\right| d \theta \quad \forall z \in B_{r}(0) .
$$

To see this, consider $h(z):=\ln (r-\bar{a} z / r)$ which is analytic in $\bar{B}_{r}(0)$ [Note that $r-\bar{a} z / r \in$ $B_{r}(r)$ for all $\left.z \in \bar{B}_{r}(0)\right]$. Since

$$
\operatorname{Re} h\left(r e^{i \phi}\right)=\ln \left|r-\bar{a} e^{i \theta}\right|=\ln \left|r e^{i \theta}-a\right|,
$$

the Poisson formula implies

$$
\ln \left|r-\frac{\bar{a} z}{r}\right|=\operatorname{Re} h(z)=\frac{1}{2 \pi} \int_{0}^{2 \pi} \operatorname{Re}\left(\frac{r e^{i \theta}+z}{r e^{i \theta}-z}\right) \operatorname{Re} h\left(r e^{i \theta}\right) d \theta
$$

and the poof is complete. 
We now discuss the Poisson-Jensen Formula for the special case where $z=0$.

Let $f$ be an analytic function in an open set containing $\bar{B}_{r}(0)$ such that $f(z) \neq 0$ for all $z \in \bar{B}_{r}(0)$, then the Poisson-Jensen Formula implies

$$
\ln |f(0)|=\frac{1}{2 \pi} \int_{0}^{2 \pi} \ln \left|f\left(r e^{i \theta}\right)\right| d \theta .
$$

Eq. (H.1) still holds if $f$ has a zero of modulus $r$. To see this let $f(z)=(z-$ $\left.r e^{i \phi}\right) g(z)$, then $\ln \left|f\left(r e^{i \theta}\right)\right|=\ln \left|g\left(r e^{i \theta}\right)\right|+\ln r+\ln \left|e^{i \theta}-e^{i \phi}\right|$. Since

$$
\int_{0}^{2 \pi} \ln \left|e^{i \theta}-e^{i \phi}\right| d \theta=\int_{0}^{2 \pi} \ln \left|e^{i \theta}-1\right| d \theta=2 \pi \ln (2)+\int_{0}^{2 \pi} \ln \sin (\theta / 2) d \theta=0,
$$

by Lemma H.1, and since Eq. (H.1) holds for $g$, we have

$$
\frac{1}{2 \pi} \int_{0}^{2 \pi} \ln \left|f\left(r e^{i \theta}\right)\right| d \theta=\frac{1}{2 \pi} \int_{0}^{2 \pi} \ln \left|g\left(r e^{i \theta}\right)\right| d \theta+\ln r=\ln |g(0)|+\ln r=\ln |f(0)| .
$$

Now suppose $f$ has a zero of modulus less than $r$, say at $a$. Following [[Con78] XI.1.2 p. 280] we put

$$
F(z):=f(z) \frac{r^{2}-\bar{a} z}{r(z-a)}
$$

then $F$ is analytic in an open set containing $\bar{B}_{r}(0)$ and $F(z) \neq 0$ for all $z \in \bar{B}_{r}(0)$. Hence Eq. (H.1) holds for $F$ and since

$$
|F(z)|=|f(z)|\left|\frac{r^{2}-\bar{a} z}{r(z-a)}\right|=|f(z)| \quad \text { for } z=r e^{i \theta},
$$

we have

$$
\frac{1}{2 \pi} \int_{0}^{2 \pi} \ln \left|f\left(r e^{i \theta}\right)\right| d \theta=\ln |F(0)|=\ln \left|\frac{1}{a} f(0) r\right| .
$$

By means of $\tilde{f}(z):=1 / f(z)$ we can generalize Eq. (H.1) in case of a pole. We thus obtain:

Jensen's Formula. Let $f \neq 0$ be a meromorphic function on $\mathbb{C}$ with zeros $a_{1}, a_{2}, \ldots$ and poles $b_{1}, b_{2}, \ldots$ repeated according to multiplicity and arranged with non decreasing moduli. If $f(0) \neq 0$, then for $r>0$ we have

$$
\ln \left(\left|\frac{b_{1} \cdots b_{n}}{a_{1} \cdots a_{m}} f(0)\right| r^{m-n}\right)=\frac{1}{2 \pi} \int_{0}^{2 \pi} \ln \left|f\left(r e^{i \theta}\right)\right| d \theta,
$$

where $a_{1}, \ldots, a_{m}$ are the zeros and $b_{1}, \ldots, b_{n}$ are the poles of $f$ in $\overline{B_{r}}(0)$.

We now go further, following [[Hol73] 9.1, p. 163] and consider the case where $f$ has a zero or a pole at the origin.

Case I. Suppose $f$ has a zero of order $k$ at 0 . Put

$$
g(z):=\left\{\begin{array}{ll}
z^{-k} f(z), & z \neq 0 \\
c, & z=0,
\end{array} \quad \text { where } c:=\frac{1}{k !} f^{(k)}(0) \neq 0 .\right.
$$


Then we may apply (H.2) to $g$ and we obtain

$$
\ln \left(\left|\frac{b_{1} \cdots b_{n}}{a_{1} \cdots a_{m}}\right| r^{m-n}\right)+\ln |c|=\frac{1}{2 \pi} \int_{0}^{2 \pi} \ln \left|f\left(r e^{i \theta}\right)\right| d \theta-k \ln r .
$$

Case II. Now suppose $f$ has a pole of order $k$ at 0 . Put

$$
g(z):=\left\{\begin{array}{ll}
z^{k} f(z), & z \neq 0 \\
c, & z=0,
\end{array} \quad \text { where } c:=\lim _{z \rightarrow 0} z^{k} f(z) \neq 0 .\right.
$$

Applying (H.2) to $g$ we find

$$
\ln \left(\left|\frac{b_{1} \cdots b_{n}}{a_{1} \cdots a_{m}}\right| r^{m-n}\right)+\ln |c|=\frac{1}{2 \pi} \int_{0}^{2 \pi} \ln \left|f\left(r e^{i \theta}\right)\right| d \theta+k \ln r .
$$

Before we combine both cases we need the following

Lemma. Let $f \neq 0$ be a meromorphic function on $\mathbb{C}$. If $n(r, 0)$ is the number of zeros of $f$ in $\bar{B}_{r}(0)$ counted according to their multiplicity, then

$$
\ln \left(\frac{r^{m}}{\left|a_{1} \cdots a_{m}\right|}\right)=\int_{0}^{r} \frac{n(t, 0)-n(0,0)}{t} d t
$$

where $a_{1}, \ldots, a_{m}$ are the zeros of $f$ in $B_{r}(0) \backslash\{0\}$. Similarly, let $n(r, \infty)$ denote the number of poles of $f$ in $\bar{B}_{r}(0)$, then

$$
\ln \left(\frac{r^{n}}{\left|b_{1} \cdots b_{n}\right|}\right)=\int_{0}^{r} \frac{n(t, \infty)-n(0, \infty)}{t} d t
$$

where $b_{1}, \ldots, b_{n}$ are the poles of $f$ in $B_{r}(0) \backslash\{0\}$.

Proof. We have

$$
\begin{aligned}
\ln \left(\frac{r^{m}}{\left|a_{1} \cdots a_{m}\right|}\right) & =m \ln r-\sum_{j=1}^{m} \ln \left|a_{j}\right|=\sum_{j=1}^{m-1} j\left(\ln \left|a_{j+1}\right|-\ln \left|a_{j}\right|\right)+m\left(\ln r-\ln \left|a_{m}\right|\right) \\
& =\sum_{j=1}^{m-1} j \int_{\left|a_{j}\right|}^{\left|a_{j+1}\right|} \frac{1}{t} d t+m \int_{\left|a_{m}\right|}^{r} \frac{1}{t} d t .
\end{aligned}
$$

For $\left|a_{j}\right| \leq t<\left|a_{j+1}\right|$ we have $j=n(t, 0)-n(0,0)$, which proves the first equation. The second equation follows similarly.

The general case. Let $f \neq 0$ be a meromorphic function on $\mathbb{C}$. Define

$$
N(r, f):=\int_{0}^{r} \frac{n(t, \infty)-n(0, \infty)}{t} d t+n(0, \infty) \ln r .
$$


[Note that if $n(0,0)>0$, then $n(0, \infty)=0$ and vice versa.] We have

$$
N(r, 1 / f)-N(r, f)=\frac{1}{2 \pi} \int_{0}^{2 \pi} \ln \left|f\left(r e^{i \theta}\right)\right| d \theta-\ln |c| .
$$

The notation uses the fact that the poles of $f$ are zeros of $1 / f$.

The characteristic function. Define $\ln ^{+} \alpha:=\max (\ln \alpha, 0)$ for $\alpha>0$. Then $\ln$ and $\ln ^{+}$are related by

$$
\ln \alpha=\ln ^{+} \alpha-\ln ^{+} \frac{1}{\alpha}, \quad \forall \alpha>0
$$

Put

$$
m(r, f):=\frac{1}{2 \pi} \int_{0}^{2 \pi} \ln ^{+}\left|f\left(r e^{i \theta}\right)\right| d \theta .
$$

By (H.1) fol. the integral is well-defined even if $f\left(r e^{i \theta_{0}}\right)=0$ for some $\theta_{0} \in[0,2 \pi]$. The characteristic function of $f$ is defined as

$$
T(r, f):=m(r, f)+N(r, f) .
$$

Now Eq. (H.3) may be written as

$$
T(r, 1 / f)=T(r, f)-\ln |c|
$$

Lemma. Let $f, g$ be meromorphic functions then

$$
T(r, f g) \leq T(r, f)+T(r, g)
$$

see [[Lev96], Lecture 2 Problem 1].

Proof. It is easy to check that $m(r, f g) \leq m(r, f)+m(r, g)$. If $z_{0}$ is a pole of $f g$, then it must be a pole of $f$ or $g$, hence $n_{f g}(t, \infty) \leq n_{f}(t, \infty)+n_{g}(t, \infty)$, whence $N(r, f g) \leq N(r, f)+N(r, g)$.

Growth of entire functions. For an entire function $f$ we define

$$
M(r, f):=\max _{|z|=r}|f(z)| \quad(r \geq 0) .
$$

By the Maximum Principle $M(r, f)=\max _{|z| \leq r}|f(z)|$, so the function $r \mapsto M(r, f)$ increases monotonically.

We finish this number with a Theorem that relates the characteristic function to the growth of an entire function.

Theorem. Let $f \neq 0$ be an entire function, then

$$
T(r, f) \leq \ln ^{+} M(r, f) \leq \frac{R+r}{R-r} T(R, f) \quad \text { for } 0<r<R .
$$

In particular (taking $R=2 r$ ) we have $\ln ^{+} M(r, f) \leq 3 T(2 r, f)$. 
Proof. (Adapted from [[Hol73] Theorem 9.4.2 p. 174]) Since $f$ has no poles we have $N(r, f)=0$, thus $T(r, f)=m(r, f)$. The left-hand inequality is thus

$$
\frac{1}{2 \pi} \int_{0}^{2 \pi} \ln ^{+}\left|f\left(r e^{i \theta}\right)\right| d \theta \leq \ln ^{+} \max _{|z|=r}|f(z)|,
$$

which is clearly true.

Choose $\phi$ such that $\left|f\left(r e^{i \phi}\right)\right|=M(r, f) \neq 0$. Then by the Poisson-Jensen Formula for $z=r e^{i \phi}$ we have

$$
\ln \left|f\left(r e^{i \phi}\right)\right|=-\sum_{k=1}^{n} \ln \left|\frac{R^{2}-\overline{a_{k}} z}{R\left(z-a_{k}\right)}\right|+\frac{1}{2 \pi} \int_{0}^{2 \pi} \operatorname{Re}\left(\frac{R e^{i \theta}+z}{R e^{i \theta}-z}\right) \ln \left|f\left(R e^{i \theta}\right)\right| d \theta,
$$

where $a_{1}, \ldots, a_{n}$ are the zeros of $f$ in $B_{R}(0)$. Since $\left|R\left(z-a_{k}\right)\right| \leq\left|R^{2}-\overline{a_{k}} z\right|$ and

$$
0 \leq \frac{R-r}{R+r} \leq \operatorname{Re}\left(\frac{R e^{i \theta}+r e^{i \phi}}{R e^{i \theta}-r e^{i \phi}}\right)=\frac{R^{2}-r^{2}}{R^{2}-2 r R \cos (\phi-\theta)+r^{2}} \leq \frac{R^{2}-r^{2}}{(R-r)^{2}}=\frac{R+r}{R-r},
$$

we have

$$
\ln M(r, f) \leq \frac{R+r}{R-r} \frac{1}{2 \pi} \int_{0}^{2 \pi} \ln ^{+}\left|f\left(R e^{i \theta}\right)\right| d \theta=\frac{R+r}{R-r} T(R, f) .
$$

Note that $\frac{R+r}{R-r} T(R, f) \geq 0$, hence $\ln ^{+} M(r, f) \leq \frac{R+r}{R-r} T(R, f)$.

The following Lemma is motivated by [[Lev96] Lecture 2 (13) fol.].

H.4 Lemma. Let $f, g$ and $h$ be entire functions, such that $f=g h \neq 0$. Suppose

$$
\max (|f(z)|,|g(z)|) \leq C e^{\tau|z|} \quad \forall z \in \mathbb{C},
$$

for some constants $C, \tau>0$. Then

$$
|h(z)| \leq C^{\prime} e^{12 \tau|z|} \quad \forall z \in \mathbb{C},
$$

for some constant $C^{\prime}$.

Proof. By assumption $C>0$. Let $r>0$, then we have $\ln M(r, h) \leq 3 T(2 r, h)=$ $3 T(2 r, f / g) \leq 3 T(2 r, f / C)+3 T(2 r, C / g)=3 T(2 r, f / C)+3 T(2 r, g / C)-3 \ln |c|$, where $c:=\frac{1}{k !} g^{(k)}(0) / C$ if 0 is a zero of $g$ of order $k$, hence

$$
\ln M(r, h) \leq 3 \ln ^{+} M(2 r, f / C)+3 \ln ^{+} M(2 r, g / C)-3 \ln |c| \leq 12 \tau r-3 \ln |c|,
$$

whence, for all $|z|=r>0$ we have

$$
|h(z)| \leq M(r, h) \leq|c|^{-3} e^{12 \tau|z|} .
$$

By continuity the inequality also holds for $z=0$. 


\section{A Note on Schur's Lemma}

Schur's Lemma is usually formulated for unitary representations of some group, here we present a useful generalization.

Let $\mathscr{H}$ be a complex separable Hilbert space and let $T$ be a nonempty subset of $L(\mathscr{H})$ such that $A \in T$ implies $A^{*} \in T$. We then say that $T$ is reducible if there exists a nontrivial closed subspace $\mathscr{M} \subset \mathscr{H}$ such that

$$
A \mathscr{M} \subset \mathscr{M} \quad \forall A \in T .
$$

In this case we say that $\mathscr{M}$ is an invariant subspace. $T$ is said to be irreducible if it is not reducible. The set

$$
\mathscr{C}:=\{C \in L(\mathscr{H}):[C, A]=0 \text { for all } A \in T\}
$$

is called the commutant of $T$.

I.1 Theorem. The following statements hold.

(a) If $\mathscr{M}$ is an invariant closed subspace for $T$, then $\mathscr{M}^{\perp}$ is invariant.

(b) Let $T$ be reducible and let $\mathscr{M}$ be a nontrivial invariant closed subspace. Let $P \in$ $L(\mathscr{H})$ denote the orthogonal projection onto $\mathscr{M}$. Then $P \in \mathscr{C}$. In particular $\mathscr{C}$ contains nontrivial operators.

(c) If $S \in \mathscr{C}$, then $S^{*} \in \mathscr{C}$.

(d) If $R, S \in \mathscr{C}$ and $\alpha, \beta \in \mathbb{C}$, then $\alpha R+\beta S \in \mathscr{C}$.

(e) If there exists an $S \in \mathscr{C}$ which is not a multiple of $I$, then $T$ is reducible.

Proof. (a) Let $\psi \in \mathscr{M}^{\perp}$. Then $\langle\phi, A \psi\rangle=\left\langle A^{*} \phi, \psi\right\rangle=0$ for all $A \in T, \phi \in \mathscr{M}$, hence $A \psi \in \mathscr{M}^{\perp}$. (b) Let $A \in T$. Then $A P=P A P$ and $A^{*} P=P A^{*} P$. Hence $A P=\left(P A^{*} P\right)^{*}=\left(A^{*} P\right)^{*}=P A$. (c) Let $A \in T$ then $A S^{*}=\left(S A^{*}\right)^{*}=\left(A^{*} S\right)^{*}=S^{*} A$. (d) This is obvious. (e) (Adapted from [[Fol95] 3.5]) $S+S^{*}$ or $i\left(S-S^{*}\right)$ is not a multiple of $I$. Since these operators are self-adjoint, (c) and (d) implies that $\mathscr{C}$ contains a selfadjoint operator $R$ which is not a multiple of $I$. By means of the spectral measure $L$ of $R$ we then have a nontrivial projection in $\mathscr{C}$. Thus there exists a nontrivial closed invariant subspace.

Statements (b) and (e) are known as a part of Schur's Lemma: T is irreducible if and only if its commutant contains only scalar multiples of the identity operator.

The notion of irreducibility then naturally applies to causal localizations $(V, U, E)$ by considering the set

$$
T:=\{V(t): t \in \mathbb{R}\} \cup\{U(g): g \in I S U(2)\} \cup\left\{E(\Delta): \Delta \in \mathscr{B}\left(\mathbb{R}^{3}\right)\right\} .
$$

Similarly the notion can be applied for relativistic localizations. 


\section{References}

[Bar54] V. Bargmann. On unitary ray representations of continuous groups. Ann. Math., 59(1):1-46, 1954.

[BGL95] P. Busch, M. Grabowski, and P.J. Lahti. Operational Quantum Physics. Springer-Verlag Berlin Heidelberg, 1995.

[BK03] N. Barat and J. C. Kimball. Localization and causality for a free particle. Physics Letters A, 308(2-3):110-115, 2003.

[BR86] Asim O. Barut and Ryszard Rączka. Theory of Group Representations and Applications. World Scientific Publishing Co Pte Ltd., 1986.

[BT53] B. Bakamjian and L. H. Thomas. Relativistic particle dynamics. ii. Phys. Rev., 92(5):1300-1310, 1953.

[Cas84] Domenico P. L. Castrigiano. Causal transformations of Wightman localizations. J. Math. Phys., 25(4):1116-1121, 1984.

[Cas11] Domenico P. L. Castrigiano. Spektraltheorie Linearer Operatoren. http: //www-m7.ma.tum.de/foswiki/pub/M7/Analysis/DomenicoCastrigiano/ STLO_MA5036.pdf, 2011.

[Con78] John B. Conway. Functions of One Complex Variable I. Springer-Verlag New York, Inc., 1978.

[Die69] J. Dieudonné. Foundations of Modern Analysis. Academic Press, 1969.

[Fol61] Leslie L. Foldy. Relativistic particle systems with interaction. Phys. Rev., 122(1):275-288, 1961.

[Fol95] Gerald B. Folland. A Course in Abstract Harmonic Analysis. CRC Press LLC, 1995.

[FW50] L. L. Foldy and S. A. Wouthuysen. On the Dirac theory of spin $1 / 2$ particles and its non-relativistic limit. Phys. Rev., 78(1):29-36, 1950.

[Heg74] G. C. Hegerfeldt. Remark on causality and particle localization. Phys. Rev. D, 10(10):3320-3321, 1974.

[Heg98] G. C. Hegerfeldt. Causality, particle localization and positivity of the energy. In Irreversibility and Causality Semigroups and Rigged Hilbert Spaces, volume 504-504 of Lecture Notes in Physics, pages 238-245. Springer Berlin Heidelberg, 1998. 
[Hol73] A. S. B. Holland. Introduction to the Theory of Entire Functions. Academic Press, Inc., 1973.

[HS65] Edwin Hewitt and Karl Stromberg. Real and Abstract Analysis. SpringerVerlag Berlin Heidelberg, 1965.

[Jor80] Thomas F. Jordan. Simple derivation of the Newton-Wigner position operator. J. Math. Phys., 21(8):2028-2032, 1980.

[Lev96] B. Ya. Levin. Lectures on Entire Functions. American Mathematical Society, 1996.

[Mac49] G. W. Mackey. Imprimitivity for representations of locally compact groups I. Proc. Natl. Acad. Sci. U.S.A., 35(9):537-545, 1949.

[Mes61] Albert Messiah. Quantum Mechanics Vol. II. Elsevier Science B.V., 1961.

[Mut78] U. Mutze. A no-go theorem concerning the cluster decomposition property of direct-interaction scattering theories. J. Math. Phys., 19(1):231-236, 1978.

[Mut84] U. Mutze. Relativistic quantum mechanics of $n$-particle systems with clusterseparable interactions. Phys. Rev. D, 29(10):2255-2269, 1984.

[PP37] M. Plancherel and G. Pólya. Fonctions entières et intégrales de fourier multiples. Commentarii Mathematici Helvetici, 9, 1936-1937.

[Pry48] M. H. L. Pryce. The mass-centre in the restricted theory of relativity and its connexion with the quantum theory of elementary particles. Proc. Roy. Soc., 195A(1040):62-81, 1948.

[Ron74] Lev I. Ronkin. Introduction to the Theory of Entire Functions of Several Variables. American Mathematical Soc., 1974.

[RS80] Michael Reed and Barry Simon. Methods of Modern Mathematical Physics I: Functional Analysis (Revised and Enlarged Edition). Academic Press, Inc., 1980 .

[RSN82] F. Riesz and B. Szőkefalvi-Nagy. Vorlesungen über Funktionalanalysis. Hochschulbücher für Mathematik. Harri Deutsch Verlag, 1982.

[Rud70] Walter Rudin. Real and Complex Analysis. McGraw-Hill, 1970.

[Rud91] Walter Rudin. Functional Analysis. McGraw-Hill, 1991.

[Sch70] Manfred Schaaf. The Reduction of the Product of Two Irreducible Unitary Representations of the Proper Orthochronous Quantummechanical Poincaré Group. Springer-Verlag, 1970. 
[Scu77] H. Scutaru. Coherent states and induced representations. Letters in Mathematical Physics, 2(2):101-107, 1977.

[SG75] K. Schulten and R.G. Gordon. Exact recursive evaluation of $3 j$ - and $6 j$ coefficients for quantum mechanical coupling of angular momenta. J. Math. Phys., 16(10):1961-1970, 1975.

[SS11] Elias M. Stein and Rami Shakarchi. Functional Analysis: Introduction to Further Topics in Analysis. Princeton University Press, 2011.

[SvN50] I. E. Segal and John von Neumann. A theorem on unitary representations of semisimple lie groups. Annals of Mathematics, Second Series, 52(3):509-517, 1950 .

[Tha92] B. Thaller. The Dirac Equation. Springer-Verlag Berlin Heidelberg, 1992.

[Var07] V.S. Varadarajan. Geometry of Quantum Theory. Springer Science+Business Media, LLC, 2007.

[Wei95] S. Weinberg. The Quantum Theory of Fields I. Cambridge University Press, 1995.

[Wei13] Eric W. Weisstein. Wigner 3j-Symbol. From MathWorld - A Wolfram Web Resource. http://mathworld.wolfram.com/Wigner3j-Symbol.html, 2013.

[Wig62] A. S. Wightman. On the localizability of quantum mechanical systems. Rev. Mod. Phys., 34:845-872, 1962.

[Yos65] Kôsaka Yosida. Functional Analysis. Springer-Verlag, 1965. 